THE OPEN BOOK SERIES 3

\title{
Poincaré Duality in Dimension 3
}

\section{Jonathan A. Hillman}

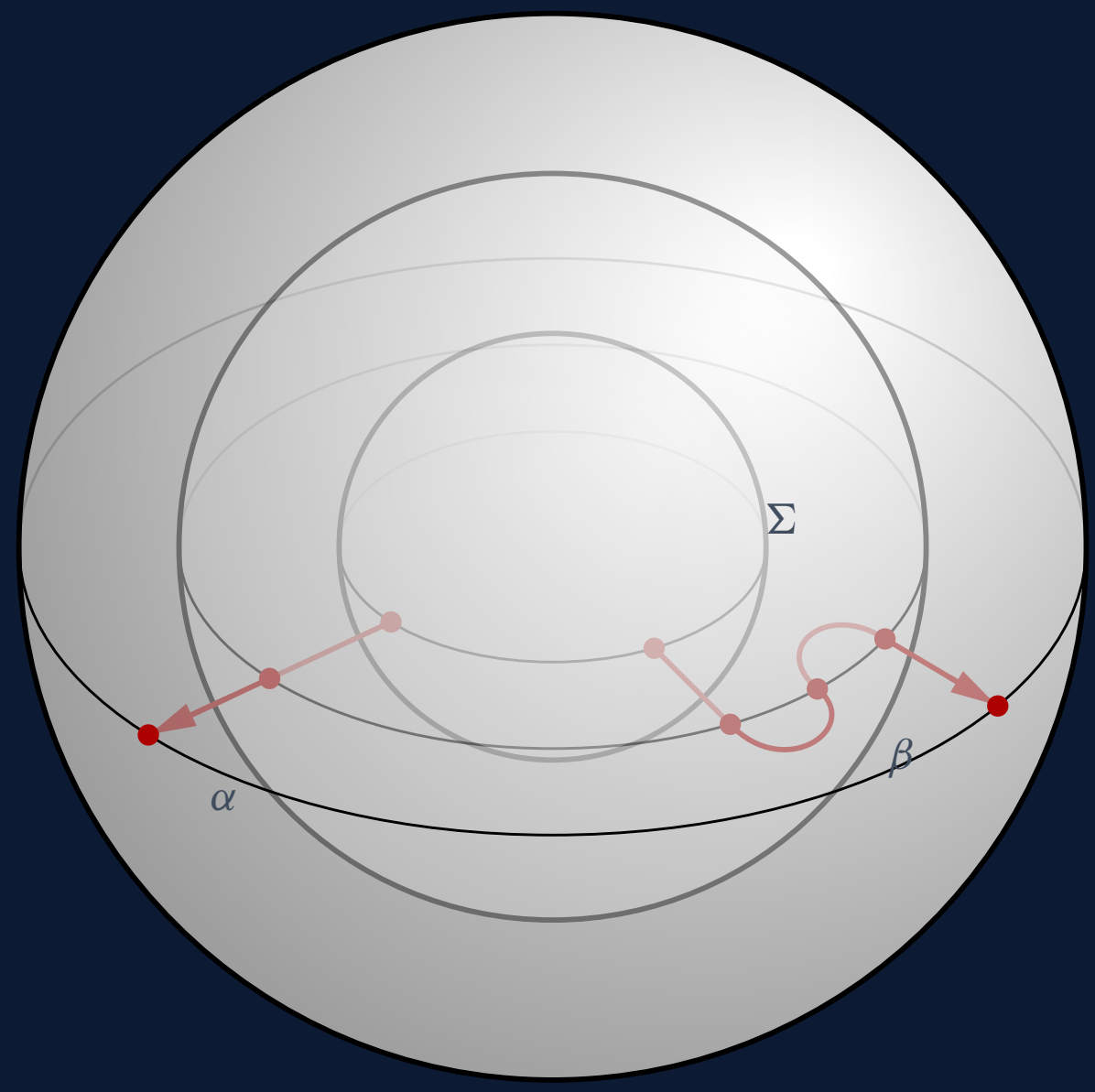

$$
\alpha . \Sigma=\beta . \Sigma=1
$$

$$
H_{1}\left(\mathbb{S}^{2} \times I, \partial\right) \approx \operatorname{Hom}\left(H_{2}\left(\mathbb{S}^{2} \times I\right), \mathbb{Z}\right)
$$



Poincaré Duality in Dimension 3

Jonathan A. Hillman 



\section{THE OPEN BOOK SERIES 3}

\section{Poincaré Duality in Dimension 3}

Jonathan A. Hillman 
Jonathan A. Hillman

The University of Sydney

The cover image is based on a design by the author.

The contents of this work are copyrighted by the author.

All rights reserved.

Electronic copies can be obtained free of charge from http://msp.org/obs/2 and printed copies can be ordered from MSP (contact@msp.org).

The Open Book Series is a trademark of Mathematical Sciences Publishers.

ISSN: 2329-9061 (print), 2329-907X (electronic)

ISBN: 978-1-935107-05-7 (print), 978-1-935107-06-4 (electronic)

First published 2020.

\section{imsp}

\section{MATHEMATICAL SCIENCES PUBLISHERS}

798 Evans Hall \#3840, c/o University of California, Berkeley CA 94720-3840 contact@msp.org 
THE OPEN BOOK SERIES 3 (2020)

Poincaré Duality in Dimension 3

https://doi.org/10.2140/obs.2020.3.1

\title{
Poincaré Duality in Dimension 3
}

\author{
Jonathan A. Hillman
}

In memory of Charles B. Thomas 



\section{Contents}

Contents vii

Preface $\quad \mathbf{x i}$

Chapter 1. Generalities $\quad 1$

1.1. Group theoretic preliminaries 1

1.2. Group rings and finiteness conditions 2

1.3. Projective homotopy of modules 3

1.4. Graphs of groups 3

1.5. Covering spaces 5

1.6. Poincaré duality 6

1.7. Poincaré duality groups 8

1.8. Poincaré duality pairs 8

1.9. Poincaré duality complexes of dimension 1 or $2 \quad 10$

1.10. Infinite cyclic covers of 4-manifolds 11

$\begin{array}{ll}\text { Chapter 2. Classification, Realization and Splitting } & \mathbf{1 3}\end{array}$

2.1. A low-dimensional simplification 13

2.2. The Classification Theorem 14

2.3. Homological triple versus algebraic 2-type 16

2.4. The Group Realization Theorem 16

2.5. Statement of the Realization Theorem of Turaev 18

2.6. Connected sum decompositions 20

2.7. Uniqueness of factorization 22

2.8. The finiteness obstruction 23

2.9. $\pi_{2}$ as a $\mathbb{Z}[\pi]$-module $\quad 24$

$\begin{array}{lll}\text { Chapter 3. The relative case } & \mathbf{2 7}\end{array}$

3.1. The basic invariants $\quad 27$

3.2. A Realization Theorem for certain pairs 29

3.3. Connected sums 33

3.4. The Algebraic Loop Theorem 34

$\begin{array}{ll}\text { 3.5. Cubes with handles } & 37\end{array}$ 
Chapter 4. The Centralizer Condition

4.1. Trees with $\infty$-vertices and the invariant $\xi \quad 39$

4.2. The action of cyclic subgroups on a $G$-tree module 41

4.3. Consequences for $\mathrm{PD}_{3}$-complexes 42

Chapter 5. Orientable $\mathrm{PD}_{3}$-complexes with $\pi$ virtually cyclic 45

5.1. Finite fundamental groups 45

5.2. Finite groups with periodic cohomology 46

5.3. Finite groups of cohomological period dividing $4 \quad 47$

5.4. A finite complex with fundamental group $S_{3}$

5.5. Self homotopy equivalences 50

5.6. Finiteness, standardness, and $\mathbb{S}^{3}$-manifolds 51

5.7. Fundamental group with two ends 51

Chapter 6. Indecomposable orientable $\mathrm{PD}_{3}$-complexes $\mathbf{5 3}$

6.1. A finite complex with group $S_{3} * \mathbb{Z} / 2 \mathbb{Z} S_{3}$

6.2. Other $\mathrm{PD}_{3}$-complexes with this group 55

6.3. Vertex groups have periodic cohomology 56

6.4. Eliminating groups with cohomological period $>4 \quad 58$

6.5. Groups with cohomological period 1, 2 or $4 \quad 59$

6.6. Extending the construction of $\S 1 \quad 61$

6.7. Homotopy types with given fundamental group 61

Chapter 7. Nonorientable $\mathrm{PD}_{3}$-complexes $\quad 63$

7.1. Nonorientable $\mathrm{PD}_{3}$-complexes with $\pi$ virtually free 63

7.2. Indecomposable nonorientable 3-manifolds 66

7.3. Indecomposable nonorientable $\mathrm{PD}_{3}$-complexes 66

$\begin{array}{lll}\text { Chapter 8. Asphericity and 3-manifolds } & \mathbf{7 3}\end{array}$

8.1. Some properties of 3-manifold groups 73

8.2. Are $\mathrm{PD}_{3}$-groups 3-manifold groups?

8.3. Some reductions 76

8.4. A compendium of frequently cited theorems 77

8.5. Mapping tori and Seifert fibred 3-manifolds 80

8.6. The groups of aspherical geometric 3-manifolds 83

Chapter 9. Centralizers, normalizers and ascendant subgroups $\quad \mathbf{8 5}$

9.1. Centralizers $\quad 85$

9.2. The maximum condition on centralizers 87

9.3. Normalizers of rank 1 abelian subgroups $\quad 88$

9.4. Normalizers of other $\mathrm{FP}_{2}$ subgroups $\quad 89$ 
9.5. Ascendant $\mathrm{FP}_{2}$ subgroups $\quad 91$

9.6. Extending Elkalla's results? 94

9.7. Splitting $\mathrm{PD}_{2}$-subgroups with nontrivial normalizers 94

$\begin{array}{lll}\text { Chapter 10. } & \text { Splitting along } \mathrm{PD}_{2} \text {-subgroups } & 97\end{array}$

10.1. Kropholler-Roller splitting theorems 97

10.2. Commensurators of $\mathrm{PD}_{2}$-subgroups 98

10.3. Kropholler's JSJ Decomposition Theorem 100

10.4. Castel's Centralizer Theorem 101

10.5. No nontrivial Baumslag-Solitar relations 105

$\begin{array}{ll}\text { Chapter 11. The Tits Alternative } & \mathbf{1 0 7}\end{array}$

11.1. The virtually Haken case 107

11.2. Profinite and pro- $p$ completions 109

11.3. Nilpotent and solvable quotients 110

11.4. $\mathrm{PD}_{3}$-groups with infinite profinite completion 112

$\begin{array}{ll}\text { 11.5. The Tits Alternative } & 115\end{array}$

11.6. Largeness 117

Chapter 12. Homomorphisms of nonzero degree 119

12.1. Automorphisms of $\mathbb{N i l}^{3}$ - and $\widetilde{S \mathbb{L}}$-groups 119

$\begin{array}{ll}\text { 12.2. Wang's partition } & 120\end{array}$

12.3. Homomorphisms with domain a graph of groups 122

12.4. Homomorphisms with solvable or Seifert domain 124

12.5. Endomorphisms and subgroups of finite index 125

$\begin{array}{lr}\text { Appendix: Some open questions } & 129\end{array}$

$\begin{array}{ll}\text { A.1. The group } & 129\end{array}$

A.2. Subgroups in general 130

$\begin{array}{ll}\text { A.3. Normal subgroups } & 131\end{array}$

A.4. Centralizers, normalizers and commensurators 131

A.5. The derived series and perfect subgroups 132

A.6. The Tits alternative 133

$\begin{array}{ll}\text { A.7. Atoroidal groups } & 133\end{array}$

$\begin{array}{lll}\text { A.8. Splitting } & 134\end{array}$

A.9. Residual finiteness, hopficity, cohopficity 134

$\begin{array}{ll}\text { A.10. Other questions } & 135\end{array}$

$\begin{array}{ll}\text { References } & 137\end{array}$

$\begin{array}{ll}\text { Index } & 145\end{array}$ 


\section{Preface}

Poincare duality complexes model the homotopy types of closed manifolds. The subject began in 1967 with a paper by C. T.C. Wall, in which such complexes were first defined, and the thesis of M.D. Spivak, which introduced the stable normal spherical fibration. The interest at the time was in providing foundations for high-dimensional surgery, but Wall's paper considered also the lowest-dimensional cases as illustrative examples, and raised the questions which are the focus of this book.

In dimensions $n=1$ and $n=2$, all $\mathrm{PD}_{n}$-complexes (Poincaré duality complexes of formal dimension $n$ ) are homotopy equivalent to $n$-manifolds, and the homotopy types are well understood. The case $n=3$ is critical in various respects. With the well-understood exception of quotients of $S^{3}$ or $S^{2} \times S^{1}$, indecomposable closed 3-manifolds are aspherical, and are homeomorphic if and only if their fundamental groups are isomorphic. These groups are $\mathrm{PD}_{3}$-groups, and it is natural to ask whether every such group arises in this way. On the other hand, in dimensions 4 and higher there is a plethora of exotic examples; indeed there are uncountably many $\mathrm{PD}_{4}$-groups, by a recent result of I. J. Leary.

Every orientable $\mathrm{PD}_{3}$-complex is a connected sum of indecomposables, which are either aspherical or have virtually free fundamental group. There are examples of the latter type which are not homotopy equivalent to 3-manifolds. The first such were constructed by R. G. Swan in another context (before the notion of $\mathrm{PD}_{n}$-complex existed). This book shall give an account of the reduction to indecomposables for $\mathrm{PD}_{3}$-complexes, and what is presently known about them. The primary interest is in the aspherical case.

$\mathrm{H}$. Hendriks showed that $\mathrm{PD}_{3}$-complexes were classified by "fundamental triples," extending work of G. A. Swarup, who in turn had reinterpreted work of C. B. Thomas on homotopy invariants for 3manifolds. This was complemented by V. G. Turaev, who determined which such triples were realized by $\mathrm{PD}_{3}$-complexes and proved splitting and unique factorization theorems. The indecomposables have been largely determined, through the work of J. S. Crisp and myself. (There remain some cases which have resisted analysis.) The work of Hendriks and Turaev has been extended to the relative case $\left(\mathrm{PD}_{3}\right.$ pairs) by B. Bleile.

The study of Poincaré duality groups has involved rather more people. F. E. A. Johnson and Wall defined a Poincaré duality group as one with a classifying space which is a Poincaré duality complex, and shortly thereafter R. Bieri and B. Eckmann gave a more algebraic definition. Eckmann, H. Müller, and P. A. Linnell showed that in dimension 2 all such groups are surface groups. Many people have contributed to the further study of Poincaré duality groups, often as an application of wider programmes in group theory. I shall mention here only the work of P. H. Kropholler and M. A. Roller on splitting $\mathrm{PD}_{n}$-groups along codimension-1 subgroups; the hyperbolization construction of $\mathrm{M}$. W. Davis, with the 
consequence that any bad behaviour in groups of cohomological dimension 2 is manifest in $\mathrm{PD}_{n}$-groups if $n \geq 4$; and most recently the use of coarse geometry by M. Kapovich and B. Kleiner.

My interest in this area began in the early 1980s, when I found a simple characterization of Seifert fibred 3-manifolds in terms of $\mathrm{PD}_{3}$-groups (later much improved by B. H. Bowditch) in the course of work on 2-knots with aspherical knot manifold. This led me to write up as an aide-memoire a list of questions about subgroups of $\mathrm{PD}_{3}$-groups, in the hope of understanding properties of 3-manifold groups through homological algebra. The QMC lecture notes of Bieri were a major guide for me then and since.

The first chapter covers the basic definitions, notation, and properties of groups; the homotopy type of modules; cell-complexes and Poincaré duality; and the known results in dimensions 1 and 2. Our general approach is to try to prove most assertions which are specifically about Poincaré duality in dimension 3, but otherwise to cite standard references for many of the major relevant supporting results, such as Bieri's work on normal subgroups of groups of finite cohomological dimension, the Algebraic Core Theorem of Kapovich and Kleiner, and the existence of JSJ decompositions for $\mathrm{PD}_{n}$-groups. (We give statements of such results in Chapters 8 and 10.)

In Chapter 2 we consider the work of Hendriks and Turaev on classification by fundamental triples, realization of invariants, and splitting theorems. While our focus is primarily on the absolute case, the potential utility of the JSJ decomposition almost obliges us to deal with the relative case. Chapter 3 gives the parallel results of Bleile for $\mathrm{PD}_{3}$-pairs, and also gives the Algebraic Loop Theorem of Crisp. Turaev showed that every $\mathrm{PD}_{3}$-complex has an essentially unique factorization as a connected sum of indecomposables. Chapter 4 follows Crisp in showing that (when orientable) these are either aspherical or have virtually free fundamental group, and gives also his restriction on centralizers of torsion elements. The next three chapters consider the case when the fundamental group $\pi$ is virtually free. The case when $\pi$ is finite has been well understood since the work of Swan, and has been of continuing interest as an aspect of the Spherical Space Form problem. We give a brief account of this case, and of the case when $\pi$ has two ends, in Chapter 5. The Centralizer Condition of Crisp is used in Chapters 6 and 7 to analyze indecomposable $\mathrm{PD}_{3}$-complexes with virtually free fundamental group. There are many examples of the latter type which are not homotopy equivalent to 3-manifolds, but the possible groups are largely known.

The remainder of the book is devoted to the aspherical case. In Chapter 8 we sketch three strategies for attempting to show that $\mathrm{PD}_{3}$-groups are 3-manifold groups, and we identify the groups of aspherical closed geometric 3-manifolds (with the exception of the geometry $\mathbb{H}^{3}$ ) and 3-dimensional mapping tori in terms of $\mathrm{PD}_{3}$-groups. We also state without proof some further results on the cohomology of groups that we shall draw upon. In Chapter 9 we consider centralizers and normalizers of $\mathrm{FP}_{2}$ subgroups, and we show that $\mathrm{PD}_{3}$-groups satisfy max- $c$ : properly increasing sequences of centralizers are finite. We show also that, in general, ascendant subgroups are usually close to being normal subgroups. The next two chapters are somewhat different in character, in that they depend more substantially on references to the original papers for many of the arguments. In Chapter 10 we state the JSJ Decomposition Theorem, and follow F. Castel in using it to further understand the centralizers of cyclic subgroups and to show that no nontrivial Baumslag-Solitar relation holds in any $\mathrm{PD}_{3}$-group. In Chapter 11 we give some of the results of $\mathrm{M}$. Boileau and $\mathrm{S}$. Boyer on the Tits alternative for almost coherent $\mathrm{PD}_{3}$-groups. We conclude in Chapter 12 by giving algebraic proofs of results of S. C. Wang on the existence of degree-1 maps between geometric 3-manifolds. 
The appendix is an updated version of my old aide-memoire on questions about 3-dimensional Poincaré duality groups and their subgroups. Most of these remain open after more than 30 years.

I would like to thank Vladimir Turaev, Michel Boileau, Steve Boyer, and my former students Bea Bleile and John Crisp for their permissions to quote extensively from some of their publications; Terry Wall for approving the reuse of the title of his Casson Fest survey article; and Peter Kropholler, Peter Scott, and Henry Wilton for their advice on some points of detail. I would also like to thank Bea and John for the experience of working with them. The opportunities to give mini-courses on Poincaré duality in low dimensions at the XVIII Encontro Brasileiro de Topologia in Águas de Lindóia (2012) and at the University of Chicago (2014), and to give a talk on $\mathrm{PD}_{3}$-groups at the conference Structure of 3-Manifold Groups at CIRM Luminy (2018), contributed to my decision to write this book.

Finally, I would like to thank Silvio Levy for his meticulous attention to my typescript and the School of Mathematics and Statistics of the University of Sydney for its support. 



\section{CHAPTER 1}

\section{Generalities}

In this chapter we shall collect basic notation, terminology and results from areas beyond our specific concern with 3-dimensional spaces. Terms used in only one chapter are usually defined there.

\subsection{Group theoretic preliminaries}

If $G$ is a group, $|G|, G^{\prime}, \zeta G$ and $\sqrt{G}$ are the order, commutator subgroup, centre and Hirsch-Plotkin radical of $G$, respectively. (In all cases considered in this book, the Hirsch-Plotkin radical is the maximal nilpotent normal subgroup.) If $H \leqslant G$ is a subgroup, $C_{G}(H)$ and $N_{G}(H)$ are the centralizer and normalizer of $H$ in $G$. We shall usually write $C_{G}(h)$ for $C_{G}(\langle h\rangle)$.

If $g \in G$, let $c_{g}$ be the automorphism of $G$ defined by $c_{g}(h)=g h g^{-1}$, for all $h \in G$. The action of $N_{G}(H)$ on $H$ by conjugation induces a homomorphism from $N_{G}(H)$ to $\operatorname{Aut}(H)$, with kernel $C_{G}(H)$. Thus if $H$ is a finite subgroup of $G$ then $C_{G}(H)$ has finite index in $N_{G}(H)$. The Normalizer Condition [Ro, 5.2.4] asserts that a proper subgroup of a nilpotent group is properly contained in its normalizer.

Two subgroups $H, K \leqslant G$ are commensurable if $H \cap K$ has finite index in each. The commensurator of $H$ in $G$ is the subgroup $\operatorname{Comm}_{G}(H)$ of $g \in G$ such that $H$ and $g H^{-1}$ are commensurable.

If $S \subseteq G$ then $\langle S\rangle$ and $\left\langle\langle S\rangle_{G}\right.$ (or just $\langle\langle S\rangle)$ are the subgroup generated by $S$ and the normal closure of $S$ in $G$, respectively.

A group virtually has some property (inherited by subgroups of finite index) if it has a subgroup of finite index with that property. If $G$ is virtually solvable let $h(G)$ be its Hirsch length. A group has max-c (the maximum condition on centralizers) if every strictly increasing sequence of centralizer subgroups in $G$ is finite. It is polycyclic if it has a finite composition series with cyclic factors.

Let $D_{\infty}=\mathbb{Z} / 2 \mathbb{Z} * \mathbb{Z} / 2 \mathbb{Z} \cong \mathbb{Z} \rtimes_{-1} \mathbb{Z} / 2 \mathbb{Z}$ be the infinite dihedral group, $F(r)$ the free group of rank $r$, and $\mathrm{Kb}=\mathbb{Z} \rtimes_{-1} \mathbb{Z}$ the Klein bottle group. If $K$ is a classical knot then $\pi K=\pi_{1}\left(S^{3} \backslash K\right)$ is the knot group. (Thus, for instance, $\pi 3_{1}$ is our notation for the group of the trefoil knot.)

Let $p, q$ be nonzero integers, and let $\mathrm{BS}(p, q)$ be the Baumslag-Solitar group with presentation $\langle x, y|$ $\left.x y^{p} x^{-1}=y^{q}\right\rangle$. When $p=1$ these groups are ascending HNN extensions, and we write also $\mathbb{Z} *_{q}=$ $\mathrm{BS}(1, q)$. (Note that $\mathrm{BS}(1,1) \cong \mathbb{Z}^{2}$ and $\mathrm{BS}(1,-1) \cong \mathrm{Kb}$.)

We shall usually use $\pi$ to denote a fundamental group, and $G$ to denote a generic group, and more particularly a $\mathrm{PD}_{3}$-group.

Our principal reference for group theory is [Ro]. 


\subsection{Group rings and finiteness conditions}

Let $R$ be a ring with an anti-involution $r \mapsto \bar{r}$. If $M$ is a left $R$-module, let $\bar{M}$ denote the conjugate right module with underlying abelian group $M$ and right module structure given by

$$
m \cdot r=\bar{r} \cdot m \quad \text { for all } r \in R \text { and } m \in M .
$$

(Similarly, if $N$ is a right $R$-module then $\bar{N}$ shall denote the conjugate left module.) If $M$ is a left $R$ module then $\operatorname{Hom}_{R}(M, R)$ is naturally a right $R$-module. Let $M^{\dagger}=\overline{\operatorname{Hom}_{R}(M, R)}$, and $\operatorname{let} \mathrm{ev}_{M}: M \rightarrow M^{\dagger \dagger}$ be the homomorphism defined by $\operatorname{ev}_{M}(m)(\lambda)=\overline{\lambda(m)}$, for all $m \in M$ and $\lambda \in M^{\dagger}$. If $M$ is a finitely generated free module then $\mathrm{ev}_{M}$ is an isomorphism.

If $G$ is a group, $\varepsilon: \mathbb{Z}[G] \rightarrow \mathbb{Z}$ and $I(G)=\operatorname{Ker}(\varepsilon)$ shall denote the augmentation homomorphism and augmentation ideal, respectively. A homomorphism $w: G \rightarrow \mathbb{Z}^{\times}=\{ \pm 1\}$ defines an anti-involution of $\mathbb{Z}[G]$ by $\bar{g}=w(g) g^{-1}$, for all $g \in G$. Let $\mathbb{Z}^{w}$ be the (two-sided) $\mathbb{Z}[G]$-module with underlying group infinite cyclic and $G$ acting via $w$.

The group $G$ is $\mathrm{FP}_{n}$ if the augmentation module has a projective resolution which is finitely generated in degrees $\leqslant n$, and is FP if it also has cohomological dimension $\operatorname{cd} G=n$. A group is almost coherent if every finitely generated subgroup is $\mathrm{FP}_{2}$ (almost finitely presentable). (In so far as our arguments are largely homological, this is more natural than the usual notion of coherence, and is implied by either coherence of the group or coherence of the group ring.)

If $G$ is $\mathrm{FP}_{2}$ then $I(G)$ has a finite presentation matrix $M$. Let $D(I(G) ; M)=\operatorname{Coker}\left(M^{\dagger}\right)$. (We shall usually write just $D I(G)$ for $D(I(G) ; M)$, for simplicity. See $\S 3$.) If $G$ has a finite presentation $\mathcal{P}$ then $\mathcal{P}$ determines such a matrix $M$, which we shall call the Fox-Lyndon matrix associated to $\mathcal{P}$, via the free differential calculus [40].

If $G$ is virtually FP it has an FP subgroup $K$ of finite index, and the rational Euler characteristic $\chi^{\mathrm{virt}}(G)=\chi(K) /[G: K]$ is well defined [Br, Chapter IX.7].

Let $C_{*}$ be a $\mathbb{Z}[G]$-chain complex with an augmentation $\varepsilon: C_{0} \rightarrow \mathbb{Z}$. An equivariant diagonal approximation for $C_{*}$ is a chain homomorphism $\Delta: C_{*} \rightarrow C_{*} \otimes_{\mathbb{Z}} C_{*}$ such that

$$
(\varepsilon \otimes 1) \Delta(c)=(1 \otimes \varepsilon) \Delta(c)=c
$$

and

$$
\Delta(g c)=(g \otimes g) \Delta(c),
$$

for all $g \in \pi$ and $c \in C_{*}$. If $C_{*}$ is a projective complex it has such a diagonal approximation, and $\Delta$ is unique up to chain homotopy.

If $G$ is a group with a normal subgroup $N$ and $A$ is a left $\mathbb{Z}[G]$-module, there is a Lyndon-HochschildSerre spectral sequence (LHSSS) for $G$ as an extension of $G / N$ by $N$, with coefficients $A$ :

$$
E_{2}=H^{p}\left(G / N ; H^{q}(N ; A)\right) \Rightarrow H^{p+q}(G ; A),
$$

the $r$-th differential having bidegree $(r, 1-r)$. (See [McC, Section 10.1].)

With one fleeting exception (in the first paragraph of $\$ 5$ of Chapter 2), all chain complexes considered shall be 0 in negative degrees. 


\subsection{Projective homotopy of modules}

Let $R$ be a ring. If $M, N$ are finitely generated left $R$-modules, two homomorphisms $f, g: M \rightarrow N$ are projectively homotopic if the difference $f-g$ factors through a free module of finite rank. The set of homotopy classes of $R$-module homomorphisms from $M$ to $N$ is an abelian group [ $M, N]$. A homomorphism $f$ is a projective homotopy equivalence if there is a homomorphism $g: N \rightarrow M$ such that $g f \sim \mathrm{id}_{M}$ and $f g \sim \mathrm{id}_{N}$.

Two $R$-modules $M$ and $N$ are freely stably isomorphic if $M_{1} \oplus R^{a} \cong N \oplus R^{b}$ for some $a, b \geqslant 0$. More generally, $M$ and $N$ are projectively stably isomorphic if $M_{1} \oplus P \cong N \oplus Q$ for some finitely generated projective $R$-modules $P$ and $Q$. Let $[M]$ and $[M]_{\mathrm{pr}}$ denote the free stable isomorphism class and projective stable isomorphism class of $M$, respectively. Tietze move considerations show that if $G$ is a group, the stable isomorphism class of $D(I(G) ; M)$ is independent of the presentation matrix $M$ for $I(G)$. Hence $[D I(G)]$ and $[D I(G)]_{\mathrm{pr}}$ are also well defined.

It is easy to see that projectively stably isomorphic modules are homotopy equivalent. The following converse combines the statements of Lemmas 2.2 and 5.1 of [147].

Lemma. Let $M$ and $N$ be $R$-modules, and $f: M \rightarrow N$ a projective homotopy equivalence. Then $f$ is the composite

$$
M \rightarrow M \oplus L \stackrel{j}{\longrightarrow} N \oplus R^{m} \rightarrow N,
$$

where $L$ is a finitely generated projective $R$-module, $m \geqslant 0$, the left- and right-hand maps are the obvious inclusion and projection (respectively), and $j$ is an isomorphism. The class $\Phi([f])=[L] \in \widetilde{K}_{0}(R)$ depends only on the homotopy class of $f$.

\subsection{Graphs of groups}

A graph of groups $(\mathcal{G}, \Gamma)$ consists of a graph $\Gamma$ with origin and target functions $o$ and $t$ from the set of edges $E(\Gamma)$ to the set of vertices $V(\Gamma)$, and a family $\mathcal{G}$ of groups $G_{v}$ for each vertex $v$ and subgroups $G_{e} \leqslant G_{o(e)}$ for each edge $e$, with monomorphisms $\phi_{e}: G_{e} \rightarrow G_{t(e)}$. (We shall usually suppress the maps $\phi_{e}$ from our notation.) In considering paths or circuits in $\Gamma$ we shall not require that the edges be compatibly oriented. We shall use terms such as "graph" and "tree" to refer to both the combinatorial object and the associated geometric realization.

The fundamental group of $(\mathcal{G}, \Gamma)$ is the group $\pi \mathcal{G}$ with presentation

$$
\left\langle G_{v}, t_{e} \mid t_{e} g t_{e}^{-1}=\phi_{e}(g) \forall g \in G_{e}, t_{e}=1 \forall e \in E(T)\right\rangle,
$$

where $T$ is some maximal tree for $\Gamma$. Different choices of maximal tree give isomorphic groups. We may assume that $(\mathcal{G}, \Gamma)$ is reduced: if an edge joins distinct vertices then the edge group is isomorphic to a proper subgroup of each of these vertex groups (i.e., that if $o(e) \neq t(e)$ then $G_{e}$ is properly contained in each of $G_{o(e)}$ and $\left.G_{t(e)}.\right)$ A graph of groups $(\mathcal{G}, \Gamma)$ is indecomposable if all edge groups are nontrivial. It is admissible if it is reduced, all vertex groups are finite or one-ended groups and all edge groups are nontrivial finite groups. An edge $e$ is a loop isomorphism at $v$ if $o(e)=t(e)=v$ and the inclusions induce isomorphisms $G_{e} \cong G_{v}$.

Lemma 1.1. Let $\pi=\pi \mathcal{G}$, where $(\mathcal{G}, \Gamma)$ is a finite graph of finite groups and $\Gamma$ is a tree. If all the edge groups are nontrivial then $\pi$ is indecomposable. 
Proof. If $\pi \cong A * B$ then $\pi$ acts without global fixed points on the Bass-Serre tree $\Upsilon$ associated to the splitting. Each finite subgroup of $\pi$ fixes a point in this tree. If $x_{o}$ and $x_{t}$ are fixed by adjacent vertex groups $G_{o(e)}$ and $G_{t(e)}$ then $G_{e}$ fixes the interval $\left[x_{o}, x_{t}\right]$ joining these points. Hence $x_{o}=x_{t}$, since edgestabilizers in $\Upsilon$ are trivial. Induction on the size of $\Gamma$ now shows that $x_{o}$ is fixed by $\pi$, contradicting the first sentence of the proof.

The argument extends easily to all finite graphs of finite groups with nontrivial edge groups.

A finitely generated group $G$ is accessible if $G \cong \pi \mathcal{G}$, where $(\mathcal{G}, \Gamma)$ is a finite graph of finitely generated groups, in which all vertex groups are finite or have one end and all edge groups are finite. Almost finitely presentable $\left(\mathrm{FP}_{2}\right)$ groups are accessible [DD, Theorem VI.6.3].

If $G$ is a finitely generated group then it has $e(G)=0,1,2$ or infinitely many ends. It is finite if and only if $e(G)=0$. If $G$ is infinite then the end module $E(G)=H^{1}(G ; \mathbb{Z}[G])$ is a free abelian group of rank $e(G)-1[\mathrm{Ge}$, Theorem 13.5.5]. It has a natural right $\mathbb{Z}[G]$-module structure.

Theorem 1.2 (End Module Theorem). Let $G=\pi \mathcal{G}$ be the fundamental group of a finite graph of finitely generated groups $(\mathcal{G}, \Gamma)$, in which all vertex groups are finite or have one end and all edge groups are finite. Let $f V$ be the set of vertices $v$ of $\Gamma$ such that $G_{v}$ is finite. Then there is a short exact sequence of right $\mathbb{Z}[G]$-modules

$$
0 \rightarrow \bigoplus_{v \in f V} \mathbb{Z}\left[G_{v} \backslash \pi\right] \stackrel{\Upsilon}{\longrightarrow} \bigoplus_{e \in E} \mathbb{Z}\left[G_{e} \backslash \pi\right] \rightarrow H^{1}(\pi ; \mathbb{Z}[G]) \rightarrow 0,
$$

where the image of a coset $G_{v} g$ in $\pi$ under $\Upsilon$ is

$$
\Upsilon\left(G_{v} g\right)=\sum_{o(e)=v} \sum_{G_{e} h \subset G_{v}} G_{e} h g-\sum_{t(e)=v} \sum_{G_{e} h \subset G_{v}} G_{e} h g,
$$

with outer sums over edges e and inner sums over cosets $G_{e} h$.

Proof. The Mayer-Vietoris sequence of Chiswell [21] gives an exact sequence

$$
0 \rightarrow \bigoplus_{v \in f V} H^{0}\left(G_{v} ; \mathbb{Z}[G]\right) \rightarrow \bigoplus_{e \in E} H^{0}\left(G_{e} ; \mathbb{Z}[G]\right) \rightarrow H^{1}(G ; \mathbb{Z}[G]) \rightarrow 0
$$

since the next term is $\bigoplus_{v \in f V} H^{1}\left(G_{v} ; \mathbb{Z}[G]\right)$, and each vertex group is finite or has one end. The cohomology groups $H^{0}(H ; \mathbb{Z}[G])$ may be identified with $\mathbb{Z}[H \backslash G]$, the right $\mathbb{Z}[G]$-module with $\mathbb{Z}$-basis the right cosets of the subgroup $H$ in $G$, if $H$ is finite, and are 0 if $H$ is infinite. The lemma follows easily.

A group $G$ splits over a subgroup $H$ if it is either a nontrivial free product with amalgamation $A *_{H} B$ where $H<A$ and $H<B$, or an HNN extension $A *_{H} \varphi$, where $H \leqslant A$ and $\varphi: H \rightarrow A$ is a monomorphism. (In other words, $G$ is the fundamental group of a graph of groups, in which the underlying graph has one edge and two or one vertices.)

Let $\mathcal{X}$ denote the class of groups $\pi \mathcal{G}$, where $(\mathcal{G}, \Gamma)$ is a finite graph of groups, in which every edge group and vertex group is infinite cyclic. These groups may also be characterized as groups of cohomological dimension 2 which have an infinite cyclic subgroup which is commensurable with all of its conjugates [95]. This class includes the Baumslag-Solitar groups and also the fundamental groups of Seifert fibred 3-manifolds with nonempty boundary.

Our references for graphs of groups are [DD] and [Se]. 


\subsection{Covering spaces}

Let $X$ be a connected cell complex and let $X^{[k]}$ denote its $k$-skeleton. Let $\widetilde{X}$ be the universal covering space of $X$. If $H$ is a normal subgroup of $G=\pi_{1}(X)$, we may lift the cellular decomposition of $X$ to an equivariant cellular decomposition of the corresponding covering space $X_{H}$. The cellular chain complex of $X_{H}$ with coefficients in a commutative ring $R$ is then a complex $C_{*}(X ; R[G / H])=C_{*}\left(X_{H} ; R\right)$ of left $R[G / H]$-modules, with respect to the action of the covering group $G / H$. A choice of lifts of the $q$-cells of $X$ determines a free basis for $C_{q}(X ; R[G / H])$, for all $q$, and so $C_{*}(X ; R[G / H])$ is a complex of free modules. If $X$ is a finite complex then $G$ is finitely presentable and these modules are finitely generated. If $X$ is finitely dominated, i.e., is a retract of a finite complex, then $G$ is again finitely presentable, and $C_{*}(X ; R[G])=C_{*}(\widetilde{X} ; R)$ is chain homotopy equivalent over $R[G]$ to a complex of finitely generated projective modules [148].

The Betti numbers of $X$ with coefficients in a field $F$ shall be denoted by $\beta_{i}(X ; F)=\operatorname{dim}_{F} H_{i}(X ; F)$ (or just $\beta_{i}(X)$, if $F=\mathbb{Q}$ ). If $G$ is a group let

$$
\begin{aligned}
v \beta(G) & =\sup \left\{\beta_{1}(H) \mid[G: H]<\infty\right\}, \\
v \beta(G ; p) & =\sup \left\{\beta_{1}\left(H ; \mathbb{F}_{p}\right) \mid[G: H]<\infty\right\} .
\end{aligned}
$$

The $i$-th equivariant homology module of $X$ with coefficients $R[G / H]$ is the left module

$$
H_{i}(X ; R[G / H])=H_{i}\left(C_{*}\left(X_{H} ; R\right)\right),
$$

which is clearly isomorphic to $H_{i}\left(X_{H} ; R\right)$ as an $R$-module, with the action of the covering group determining its $R[G / H]$-module structure. The $i$-th equivariant cohomology module of $X$ with coefficients $R[G / H]$ is

$$
H^{i}(X ; R[G / H])=H^{i}\left(\operatorname{Hom}_{R[G / H]}\left(C_{*}(X ; R[G / H]), R[G / H]\right)\right) .
$$

(Since the cochain groups $\operatorname{Hom}_{R[G / H]}\left(C_{*}(X ; R[G / H]), R[G / H]\right)$ are right $R[G / H]$-modules, this is also a right module.) More generally, if $A$ and $B$ are right and left $R[G / H]$-modules (respectively) let

$$
\begin{aligned}
H_{j}(X ; A) & =H_{j}\left(A \otimes_{R[G / H]} C_{*}(X ; R[G / H])\right), \\
H^{n-j}(X ; B) & =H^{n-j}\left(\operatorname{Hom}_{R[G / H]}\left(C_{*}(X ; R[G / H]), B\right)\right) .
\end{aligned}
$$

(These are $R$-modules, with no natural $R[G]$-module structure, unless $A$ or $B$ is a bimodule.) There is a universal coefficient spectral sequence relating equivariant homology and cohomology:

$$
E_{2}^{p q}=\operatorname{Ext}_{R[G / H]}^{q}\left(H_{p}(X ; R[G / H]), R[G / H]\right) \Rightarrow H^{p+q}(X ; R[G / H]),
$$

with $r$-th differential $d_{r}$ of bidegree $(1-r, r)$. (See $[\mathrm{McC}]$.)

If $M$ is a cell complex, $c_{M}: M \rightarrow K\left(\pi_{1}(M), 1\right)$ denotes the classifying map for the fundamental group.

A finite presentation $\mathcal{P}$ for a group $G$ determines a finite 2-complex $K(\mathcal{P})$ with one 0-cell and with

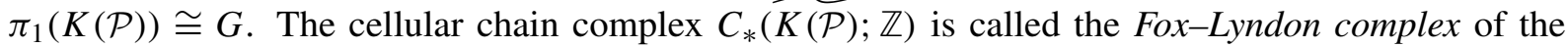
presentation; the second differential is given by the Fox-Lyndon matrix associated to $\mathcal{P}$. 


\subsection{Poincaré duality}

$\mathrm{A} \mathrm{PD}_{n}$-space is a space homotopy equivalent to a cell complex which satisfies Poincaré duality of formal dimension $n$ with local coefficients. (We shall give a more precise definition in the following paragraphs.) It is a $\mathrm{PD}_{n}$-complex if it is finitely dominated. Poincaré duality complexes were introduced by Wall to model the homotopy types of closed manifolds [150]. However, they also arise in situations where the geometry does not immediately provide a corresponding manifold. (See $\$ 10$ of this chapter for an example.)

Let $P$ be a connected cell complex with $\pi_{1}(P)=\pi$, and let $w: \pi \rightarrow \mathbb{Z}^{\times}$be a homomorphism. If $B$ is a left $\mathbb{Z}[\pi]$-module define a cochain complex $C^{*}(P ; B)$ by

$$
C^{q}(P ; B)=\operatorname{Hom}_{\mathbb{Z}[\pi]}\left(C_{q}(P ; \mathbb{Z}[\pi]), B\right) .
$$

In general this is just a complex of abelian groups, unless $B$ is a bimodule.

The space $P$ satisfies Poincaré duality of formal dimension $n$ with orientation character $w$ if there is a chain $x$ in $C_{n}(P ; \mathbb{Z}[\pi])$ such that $1 \otimes x \in \mathbb{Z}^{w} \otimes_{\mathbb{Z}[\pi]} C_{n}(P ; \mathbb{Z}[\pi])$ is an $n$-cycle for $\mathbb{Z}^{w} \otimes_{\mathbb{Z}[\pi]} C_{*}(P ; \mathbb{Z}[\pi])$ and cap product with $1 \otimes x$ defines (ZZZ-linear) chain homotopy equivalences

$$
\varphi \mapsto \varphi \cap x: C^{n-*}(P ; B) \simeq \bar{B} \otimes_{\mathbb{Z}[\pi]} C_{*}(P ; \mathbb{Z}[\pi]),
$$

for all coefficient modules $B$. This cap product is defined as follows. Let $\Delta$ be an equivariant diagonal approximation for $C_{*}(P ; \mathbb{Z}[\pi])$, and suppose that $\Delta(x)=\sum y_{i} \otimes z_{n-i}$ with $y_{i}$ and $z_{i}$ in $C_{i}(P ; \mathbb{Z}[\pi])$. Then

$$
\varphi \cap x=\varphi\left(y_{q}\right) \otimes_{\mathbb{Z}[\pi]} z_{n-q}
$$

for all $\varphi \in C^{q}(P ; B)$. The chain homotopy class of $\cap x$ does not depend on the choice of diagonal approximation or chain $x$ representing $[1 \otimes x]$. Hence Poincaré duality gives rise to (ZZ-linear) isomorphisms from $H^{j}(P ; B)$ to $H_{n-j}(P ; \bar{B})$. Note in particular that

$$
H^{n}(P ; B) \cong H_{0}(P ; \bar{B}) \cong \bar{B} \otimes_{\mathbb{Z}[\pi]} \mathbb{Z} \cong \mathbb{Z}^{w} \otimes_{\mathbb{Z}[\pi]} B
$$

as abelian groups. (See [Ra, Section 4.5] for more details.)

We shall call such a complex $P$ a $\mathrm{PD}_{n}$-space. The image $[P]$ of $x$ generates $H_{n}\left(P ; \mathbb{Z}^{w}\right) \cong \mathbb{Z}$, and is called a fundamental class for $P$. While it is easy to extend the definition to complexes with more than one component, and it is essential to do so when we consider boundaries of $\mathrm{PD}_{n}$-pairs, we shall assume without further comment that all $\mathrm{PD}_{3}$-spaces considered in this book are connected.

Homology commutes with direct limits of coefficient modules. Since $H^{j}(P ; B) \cong H_{n-j}(P ; \bar{B})$, for all left $\mathbb{Z}[\pi]$-modules $B$, cohomology also commutes with such direct limits. Therefore $C_{*}(P ; \mathbb{Z}[\pi])$ is chain homotopy equivalent to a finite projective $\mathbb{Z}[\pi]$-complex [148]. In particular, $\pi$ is $\mathrm{FP}_{2}$. The $\mathrm{PD}_{n}$-space $P$ is finite if $C_{*}(P ; \mathbb{Z}[\pi])$ is $\mathbb{Z}[\pi]$-chain homotopy equivalent to a finite free $\mathbb{Z}[\pi]$-complex. A $\mathrm{PD}_{n}$-space $P$ is a $\mathrm{PD}_{n}$-complex if and only if $\pi$ is finitely presentable [148]. Finite $\mathrm{PD}_{n}$-spaces with $\pi$ finitely presentable are homotopy equivalent to finite complexes. (Thus "finite $\mathrm{PD}_{n}$-complex" is unambiguous.) 
If $P$ is a finite complex or, more generally, if we assume a priori that $C_{*}(P ; \mathbb{Z}[\pi])$ is chain homotopy equivalent to a complex $C_{*}$ of finitely generated projective modules, then to show that $P$ satisfies Poincaré duality of formal dimension $n$ with orientation character $w$ it is enough to assume that $\cap x$ induces a chain homotopy equivalence

$$
-\cap x: C^{n-*}(P ; \mathbb{Z}[\pi]) \simeq C^{n-*}=C_{n-*}^{\dagger} \rightarrow C_{*} \simeq C_{*}(P ; \mathbb{Z}[\pi]) .
$$

For if $C_{q}$ is a finitely generated projective module there are natural ( $\mathbb{Z}$-linear) isomorphisms

$$
\operatorname{Hom}_{\mathbb{Z}[\pi]}\left(C_{q}, B\right) \cong \operatorname{Hom}_{\mathbb{Z}[\pi]}\left(C_{q}, \mathbb{Z}[\pi]\right) \otimes_{\mathbb{Z}[\pi]} B
$$

for any left $\mathbb{Z}[\pi]$-module $B$, and hence

$$
C^{q}(P ; B) \cong \bar{B} \otimes_{\mathbb{Z}[\pi]} C^{q}(P ; \mathbb{Z}[\pi])
$$

Thus we may get chain homotopy equivalences with general coefficients by taking tensor products.

Lemma 1.3. Let $P$ be a connected cell complex with $\pi_{1}(X)=\pi$, and let $\sigma$ be a normal subgroup of finite index in $\pi$. Then $P$ is a $\mathrm{PD}_{n}$-space if and only if $P_{\sigma}$ is a $\mathrm{PD}_{n}$-space. Moreover, if $[X]$ is a fundamental class for $X$ then the transfer $\operatorname{tr}[X]$ is a fundamental class for $X_{\sigma}$.

Proof. Since $\widetilde{P}_{\sigma}=\widetilde{P}$ we have $C_{*}\left(P_{\sigma} ; \mathbb{Z}[\sigma]\right)=\left.C_{*}(P ; \mathbb{Z}[\pi])\right|_{\sigma}$, and there is a natural isomorphism $\left.C^{*}\left(P_{\sigma} ; \mathbb{Z}[\sigma]\right) \cong C^{*}(P ; \mathbb{Z}[\pi])\right|_{\sigma}$, since $[\pi: \sigma]$ is finite. The lemma then follows from the functoriality of the cap product.

Let $w=w_{1}(P)$ and $\pi^{+}=\operatorname{Ker}(w)$, and let $P^{+}=P_{\pi^{+}}$be the associated covering space. If $H \leqslant \pi$ then we shall write $H^{+}=H \cap \pi^{+}$. It is convenient to say that such a subgroup $H$ is orientable if $H=H^{+}$. (This usage depends upon the orientation character $w$.) Let $\mathbb{Z} / 2 \mathbb{Z}^{-}$denote a subgroup of order two on which $w \neq 1$. If $P$ and $Q$ are $\operatorname{PD}_{n}$-spaces then $f: P \rightarrow Q$ has degree \pm 1 if $w_{1}(P)=w_{1}(Q) \circ \pi_{1}(f)$ and $H_{n}\left(f^{+}\right)$is an isomorphism.

A group $G$ is a $\mathrm{PD}_{n}$-group if and only if $K(G, 1)$ is a $\mathrm{PD}_{n}$-space. (A purely algebraic definition is given in the next section.) For every $n \geqslant 4$ there are $\mathrm{PD}_{n}$-groups which are not finitely presentable [24]. It is not known whether there are such examples when $n=3$, and it is for this reason that we have defined $\mathrm{PD}_{n}$-spaces as well as $\mathrm{PD}_{n}$-complexes. (See also [87].)

A finite $\mathrm{PD}_{n}$-complex $P$ is simple if the chain homotopy equivalence $C^{n-*}(P ; \mathbb{Z}[\pi]) \simeq C_{*}(P ; \mathbb{Z}[\pi])$ given by cap product has Whitehead torsion 0 in $\mathrm{Wh}(\pi)$. (In general, there is a well-defined torsion $\tau(P) \in \mathrm{Wh}(\pi)$.) Closed connected PL $n$-manifolds are simple finite $\mathrm{PD}_{n}$-complexes, but there are 1connected $\mathrm{PD}_{n}$-complexes which are not homotopy equivalent to manifolds, for all $n \geqslant 4$ [150].

Wall showed that every $\mathrm{PD}_{n}$-complex is homotopy equivalent to an $n$-dimensional complex, and in all dimensions except $n=3$ we may assume that there is a single $n$-cell. When $n=3$ we may write $P$ as a union $P=Y \cup e^{3}$, where $Y$ is homologically 2-dimensional [150, Theorem 2.4]. (Thus the exceptional case relates to the Eilenberg-Ganea Conjecture.) We shall say that a finite $\mathrm{PD}_{3}$-complex $P$ is standard if $P \simeq Y \cup e^{3}$, where $Y$ is a finite 2-complex [Jo].

The top cell is essentially unique, and so there is a well-defined connected sum, for oriented $\mathrm{PD}_{n^{-}}$ complexes [150, Corollary 2.4.1]. 


\subsection{Poincaré duality groups}

The notion of Poincaré duality group of dimension $n$ (or $\mathrm{PD}_{n}$-group, for short) is an algebraic analogue of the notion of aspherical $n$-manifold. A finitely presentable group $G$ is a $\mathrm{PD}_{n}$-group in the sense of Johnson and Wall if $K(G, 1)$ is homotopy equivalent to a $\mathrm{PD}_{n}$-complex [78]. Bieri and Eckmann gave an alternative purely algebraic formulation: a group $G$ is a $\mathrm{PD}_{n}$-group if it is FP (the augmentation $\mathbb{Z}[G]$-module $\mathbb{Z}$ has a finite projective resolution), $H^{i}(G ; \mathbb{Z}[G])=\operatorname{Ext}_{\mathbb{Z}[G]}^{p}(\mathbb{Z}, \mathbb{Z}[G])$ is 0 for $i<n$ and $H^{n}(G ; \mathbb{Z}[G])$ is infinite cyclic as an abelian group [Bi; 8]. These conditions hold if and only if $K(G, 1)$ is an (aspherical) $\mathrm{PD}_{n}$-space. The dualizing module $H^{n}(G ; \mathbb{Z}[G])$ is a right $\mathbb{Z}[G]$-module, with $G$-action determined by a homomorphism $w=w_{1}(G): G \rightarrow \mathbb{Z}^{\times}$. The group is orientable (or is a $\mathrm{PD}_{n}^{+}$-group) if $w$ is trivial, i.e., if $H^{n}(G ; \mathbb{Z}[G])$ is isomorphic to the augmentation module $\mathbb{Z}$. (See [Bi].)

The only $\mathrm{PD}_{1}$-group is $\mathbb{Z}$. Eckmann, Linnell and Müller showed that every $\mathrm{PD}_{2}$-group is the fundamental group of an aspherical closed surface. (See [DD, Chapter VI].) Bowditch has since found a much stronger result.

Theorem (Bowditch [15]). Let $G$ be an $\mathrm{FP}_{2}$ group and $F$ a field. Then $G$ is virtually the fundamental group of an aspherical closed surface if and only if $H^{2}(G ; F[G])$ has a 1-dimensional $G$-invariant subspace.

Bowditch used ideas from geometric group theory to show that any such group acts properly discontinuously on $\mathbb{E}^{2}$ or $\mathbb{M}^{2}$, and so is commensurable with a surface group [15].

This theorem applies if $H^{2}(G ; \mathbb{Z}[G])$ is infinite cyclic, for then its image in $H^{2}\left(G ; \mathbb{F}_{2}[G]\right)$ under reduction modulo 2 is such a subspace.

$\mathrm{PD}_{n}$-groups are FP, and thus finitely generated, but there are examples which are not finitely presentable, for all $n \geqslant 4$ [24]. Indeed, there are uncountably many [102, Theorem 18.1]. Whether there are $\mathrm{PD}_{3}$-groups which are not finitely presentable remains unknown. (The case $n=3$ is critical; there are $\mathrm{PD}_{n}$-groups with all sorts of bad behaviour when $n>3$. See [24].)

It is still an open question whether every finitely presentable $\mathrm{PD}_{n}$-group is the fundamental group of a closed $n$-manifold. (This is one aspect of the circle of ideas around the Novikov Conjecture.)

Our references for cohomological group theory are $[\mathrm{Bi}]$ and $[\mathrm{Br}]$.

\subsection{Poincaré duality pairs}

The notion of $\mathrm{PD}_{n}$-space can be elaborated to consider pairs (corresponding to manifolds with boundary). Such $\mathrm{PD}_{n}$-pairs arise naturally even when the primary interest is in the absolute case.

Let $P$ be a connected cell complex with $\pi_{1}(P)=\pi$. If $Y$ is a subcomplex of $P$ then $(P, Y)$ is a $\mathrm{PD}_{n^{-}}$ pair with orientation character $w_{1}(P)=w: \pi \rightarrow \mathbb{Z}^{\times}$and fundamental class $[P, Y]$ in $H_{n}\left(P, Y ; \mathbb{Z}^{w}\right)$ if each component $Y_{i}$ of $Y$ is a $\mathrm{PD}_{n-1}$-space with orientation character $\left.w\right|_{Y_{i}}$ and fundamental class the image of $[P, Y]$ in $H_{n-1}\left(Y_{i} ; \mathbb{Z}^{w}\right)$ under the connecting homomorphism, and there is a chain $x \in C_{n}(P, Y ; \mathbb{Z}[\pi])$ such that $1 \otimes x$ is an $n$-cycle representing $[P, Y]$ and cap product with $x$ induces a chain homotopy equivalence

$$
-\cap x: C^{n-*}(P ; \mathbb{Z}[\pi]) \rightarrow C_{*}(P, Y ; \mathbb{Z}[\pi]) .
$$

(Cap product $-\cap x: C^{n-*}(P, Y ; \mathbb{Z}[\pi]) \rightarrow C_{*}(P ; \mathbb{Z}[\pi])$ is then also a chain homotopy equivalence.) We shall also say that $(P, Y)$ satisfies Poincaré-Lefshetz duality. We shall usually write $\partial P$ for $Y$ and $\partial \widetilde{P}$ 
for the preimage of $Y$ in $\widetilde{P}$. (The components of $\partial \widetilde{P}$ need not be 1-connected.) See [87] for a detailed exposition of relative duality, and the necessity of some hypothesis on the boundary subcomplex.

$\mathrm{A} \mathrm{PD}_{n}$-pair of groups is the analogue of an aspherical compact manifold with $\pi_{1}$-injective, aspherical boundary components. This notion was introduced by Bieri and Eckmann, and arises in connection with JSJ decompositions of $\mathrm{PD}_{3}$-groups. Such a pair consists of a group $G$ of finite cohomological dimension and a finite set $\mathcal{S}$ of conjugacy classes of monomorphisms $\sigma: S \rightarrow G$ (for various groups $S$ ) satisfying a modified form of Poincaré-Lefshetz duality [8].

We shall use also a reformulation given in [DD, Chapter 7]. If $G$ is a group and $\Omega$ is a left $G$-set, let $\mathbb{Z}[\Omega]$ be the left $\mathbb{Z}[G]$-module with basis $\Omega$ as an abelian group. Let $\varepsilon_{\Omega}: \mathbb{Z}[\Omega] \rightarrow \mathbb{Z}$ be the homomorphism given by $\varepsilon_{\Omega}(u)=1$, for all $u \in \Omega$, and let

$$
\Delta(G, \Omega)=\operatorname{Ker}\left(\varepsilon_{\Omega}\right)
$$

If $M$ is a left $\mathbb{Z}[G]$-module and $k \in \mathbb{Z}$, let

$$
H^{k}(G, \Omega ; M)=H^{k-1}\left(G ; \operatorname{Hom}_{\mathbb{Z}[G]}(\Delta(G, \Omega), M)\right) .
$$

Then $(G, \Omega)$ is a $\mathrm{PD}_{n}$-pair of groups, with ambient group $G$, if $G$ is $\mathrm{FP}, H^{i}(G, \Omega ; \mathbb{Z}[G])=0$ for $i<n$ and $H^{n}(G, \Omega ; \mathbb{Z}[G]) \cong \mathbb{Z}$. The $G$-action on the latter group determines the orientation character $w=w_{(G, \Omega)}: G \rightarrow \mathbb{Z}^{\times}$.

If we use $w$ to define conjugate right $\mathbb{Z}[G]$-modules $\bar{M}$, there are natural isomorphisms $H^{k}(G, \Omega ; M) \cong$ $H_{n-k}(G ; \bar{M})$ for all left modules $M$ and $k \in \mathbb{Z}$, given by cap product with a class in $H_{n}\left(G ; \mathbb{Z}^{w}\right)$.

The boundary $\Omega$ has finitely many $G$-orbits, and the point stabilizers are $\mathrm{PD}_{n-1}$-groups $[\mathrm{DD}$, Theorem V.7.11]. We shall call these subgroups the boundary components of the pair. If $\Omega=\varnothing$ then $G$ is a $\operatorname{PD}_{n^{-}}$ group; otherwise $\mathrm{cd} G=n-1$.

If $(P, \partial P)$ is a $\mathrm{PD}_{n}$-pair with $P$ aspherical and $\pi_{1}$-injective, aspherical boundary components then $\left(\pi, \pi_{0}(\partial \widetilde{P})\right)$ is a $\mathrm{PD}_{n}$-pair of groups.

We may recover the Bieri-Eckmann approach by taking $\mathcal{S}$ to be the set of conjugacy classes of embeddings of boundary components. There may be repetitions, the simplest example being the fundamental group system of the interval $[0,1]$ with its boundary, with $G=1$ and $|\Omega|=2$. (See also Lemmas 8.6 and 10.3 below.)

If $H$ is a subgroup of finite index in $G$ there is an induced $\mathrm{PD}_{n}$-pair of groups $\left(H, \Omega_{H}\right)$. The pair $(G, \Omega)$ is atoroidal if every virtually polycyclic subgroup of $G$ of Hirsch length $n-1$ fixes some point of $\Omega$, but $G$ is not itself virtually polycyclic. It is of Seifert type if $G$ has a normal, virtually polycyclic subgroup $N$ of Hirsch length $h(N)=n-2$. The boundary components are then virtually polycyclic subgroups of Hirsch length $n-1$. In particular, a $\mathrm{PD}_{3}$-group $G$ with no abelian subgroup of rank $>1$ is atoroidal, while one with an infinite cyclic normal subgroup is of Seifert type.

Prompted by the Algebraic Core Theorem of [81], we define an open $\mathrm{PD}_{n}$-group to be a countable group $G$ of cohomological dimension $\leqslant n-1$ such that if $H$ is a nontrivial FP subgroup with $H^{s}(H ; \mathbb{Z}[H])=0$ for $s<n-1$ then $H$ is the ambient group of a $\operatorname{PD}_{n}$-pair $(H, \Omega)$. Every subgroup of infinite index in a $\mathrm{PD}_{3}$-group $G$ is an open $\mathrm{PD}_{3}$-group in our sense, by the Algebraic Core Theorem. (The analogies are precise if $n=2$, but these definitions are too broad when $n \geqslant 4$. We shall consider only $n=2$ or 3 ). 


\subsection{Poincaré duality complexes of dimension 1 or 2}

In the lowest dimensions the the modelling of $n$-manifolds by $\mathrm{PD}_{n}$-complexes is precise: every $\mathrm{PD}_{n}$ complex is homotopy equivalent to $S^{1}$ or to a closed surface, when $n=1$ or 2 , and two such manifolds are homeomorphic if and only if their fundamental groups are isomorphic. Similarly, proper PD $_{n}$-pairs in these dimensions are equivalent to the interval or to a bounded surface.

It is easy to see that a $\mathrm{PD}_{1}$-complex $X$ must be aspherical, $\pi=\pi_{1}(X)$ has two ends and $\mathrm{cd} \pi=1$. Let $G$ be a $\mathrm{PD}_{1}$-group. Then $\operatorname{cd} G=1$, so $G$ is torsion-free, and $H^{1}(G ; \mathbb{Z}[G]) \cong \mathbb{Z}$, so $G$ has two ends. Therefore $G \cong \mathbb{Z}$ and $G$ is orientable. Alternatively, $G$ is free of finite rank $r$, and so $H_{1}(G ; \mathbb{Z}) \cong \mathbb{Z}^{r}$. Hence $r=1$, i.e., $G \cong \mathbb{Z}$, and $G$ is orientable.

Here is an argument not requiring cohomological characterizations of ends or free groups. If $G$ is a $\mathrm{PD}_{1}$-group and $w=w_{1}(G)$ then $H_{1}(G ; \mathbb{Z}) \cong H^{0}\left(G ; \mathbb{Z}^{w}\right)$. If $w=w_{1}(G) \neq 0$ then $H_{1}(G ; \mathbb{Z}) \neq 0$ but $H^{0}\left(G ; \mathbb{Z}^{w}\right)=0$. This is a contradiction, so $w=0$ and $G$ is orientable. Hence $H_{1}(G ; \mathbb{Z}) \cong H_{0}(G ; \mathbb{Z})=\mathbb{Z}$, and so there is an epimorphism $\phi: G \rightarrow \mathbb{Z}$. Let $K=\operatorname{Ker}(\phi)$, and let $M$ be a left $K$-module. Let $W=\operatorname{Hom}_{\mathbb{Z}[K]}(\mathbb{Z}[G], M)$ be the coinduced left $G$-module, and let $\bar{W}$ be the conjugate right $G$-module. Then $H^{1}(K ; M) \cong H^{1}(G ; W)$, by Shapiro's Lemma. This in turn is isomorphic to $H_{0}(G ; \bar{W})$, by Poincaré duality, and is easily seen to be 0 . Therefore cd $K=0$ and so $K$ is trivial; see [Br, Exercise VIII.2.1]. Therefore $\phi$ is an isomorphism, and so $G \cong \mathbb{Z}$.

Every $\mathrm{PD}_{2}$-complex $X$ is homotopy equivalent to a closed surface. While this was expected to be so, the proofs are not easy. If the fundamental group is finite then $X$ is homotopy equivalent to one of $S^{2}$ or the real projective plane $\mathbb{R} \mathbb{P}^{2}[150]$. All others are aspherical. Eckmann and Müller showed that every $\mathrm{PD}_{2}$ complex with $\chi(X) \leqslant 0$ is homotopy equivalent to a closed surface, by first proving the corresponding result for $\mathrm{PD}_{2}$-pairs with nonempty boundary and then showing that every $\mathrm{PD}_{2}$-group splits over a copy of $\mathbb{Z}$ [32]. The argument involves delicate combinatorial group theory. Shortly afterwards, Eckmann and Linnell showed that there is no aspherical $\mathrm{PD}_{2}$-complex $X$ with $\chi(X)>0$ [31]. (See [DD, Chapter VI] for an exposition of this work.) Much later Bowditch proved the theorem cited in $\$ 7$ above, which must be close to the optimal characterization of virtual $\mathrm{PD}_{2}$-groups [15]. However, we need the relative case also. See also [85] for a succinct characterization based on geometric group theory.

Higher dimensional considerations suggest another, more topological strategy, which can be justified a posteriori. If $X$ is an orientable $\mathrm{PD}_{2}$-space then there is a degree-1 map $f: M \rightarrow X$ with domain a closed orientable surface. Choose compatible basepoints $m_{o}$ and $x_{o}=f\left(m_{o}\right)$, and let $\pi=\pi_{1}\left(X, x_{o}\right)$ and $f_{*}=\pi_{1}(f)$. Then

$$
f \text { is a homotopy equivalence } \Leftrightarrow \operatorname{Ker}\left(f_{*}\right)=1 \Leftrightarrow \chi(M)=\chi(X) \text {. }
$$

If $\operatorname{Ker}\left(f_{*}\right)$ contains the class of a non-separating simple closed curve $\gamma$ we may reduce $|\chi(M)|$ by surgery on $\gamma$. If $X$ is also a closed orientable surface and $\chi(M)<\chi(X)$ then there is a separating nontrivial simple closed curve $\gamma$ such that $M \backslash \gamma=M_{0} \sqcup M_{1}$, where $\left.f\right|_{M_{0}} \sim *$ and $\left.f\right|_{M_{1}}$ has degree 1 [34]. Since any simple closed curve in $M_{0}$ is in $\operatorname{Ker}\left(\pi_{1}(f)\right)$, there is also a non-separating curve $\gamma \subset M$ with image in the kernel of $\pi_{1}(f)$. After finitely many iterations we obtain a degree-1 map $\hat{f}: \hat{M} \rightarrow X$, with $\chi(\hat{M})=\chi(X)$. Such a map must be a homotopy equivalence. However it seems that Edmonds' argument requires the codomain $X$ to also be a 2-manifold, which is what we want to prove! Can we avoid a vicious circle? 


\subsection{Infinite cyclic covers of 4-manifolds}

In dimensions other than $n=4$ or 5 there are good geometric fibration theorems, showing that a map $f: M^{n} \rightarrow S^{1}$ such that $\pi_{1}(f)$ is an epimorphism is homotopic to the projection of a fibre bundle (and so $M$ is a mapping torus) if and only if the homotopy fibre is suitably finite and a Whitehead torsion is 0 , by the work of Stallings $(n=3)$ and Farrell $(n \geqslant 6)$. (When $n=5$ this holds also topologically, if the Disc Embedding Conjecture holds over $\pi_{1}(M)$.)

In dimension 4 there is the following analogue, which illustrates how $\mathrm{PD}_{3}$-complexes arise in a natural way.

Theorem [72; Hi]. Let $M$ be a closed 4-manifold such that $\pi=\pi_{1}(M)$ has a finitely generated normal subgroup $v$ with $\pi / v \cong \mathbb{Z}$. Then $\chi(M) \geqslant 0$. Moreover:

(1) $\chi(M)=0 \Leftrightarrow M_{v}$ is a $\mathrm{PD}_{3}$-space.

(2) If $\chi(M)=0$, then $M$ is aspherical $\Leftrightarrow v$ has one end.

(3) If $M$ is aspherical, then $\chi(M)=0 \Leftrightarrow v$ is $\mathrm{FP}_{2}$.

The proof can be simplified if $v$ is finitely presentable, and $M_{v}$ is then homotopy equivalent to a $\mathrm{PD}_{3}$ complex. If $M \simeq M_{v} \times S^{1}$ then $M_{v}$ must be finite, since $n$-manifolds are simple $\mathrm{PD}_{n}$-complexes [118]. However, the arguments of [52] give such examples with $v$ finite, but for which $M_{v}$ is not homotopy equivalent to a 3-manifold.

The argument for the above theorem is algebraic, and so it holds also for $M$ a $\mathrm{PD}_{4}$-space. Work of Gottlieb and Quinn shows that if $f: E \rightarrow B$ is a fibration with homotopy fibre $F$ and the base $B$ is a $\mathrm{PD}_{r}$-complex, then $F$ is a $\mathrm{PD}_{n-r}$-complex if and only if it is finitely dominated and $E$ is a $\mathrm{PD}_{n}$-complex. However, when $B=S^{1}$ and $n=4$ these hypotheses are stronger than those in the theorem. 



\section{CHAPTER 2}

\section{Classification, Realization and Splitting}

This chapter is based on the work of Hendriks and Turaev [58; 145; 147], and is central to the first half of this book. We begin with a simplification of the definition of $\mathrm{PD}_{3}$-complex. In $\$ 2$ we define the fundamental triple, and follow Hendriks in showing that it is a complete invariant for the homotopy type of a $\mathrm{PD}_{3}$-complex. There is an alternative system of invariants, involving the first nonzero $k$-invariant, and in $\S 3$ we ask how these two systems may be related. The Realization Theorem of Turaev characterizes the fundamental triples realized by $\mathrm{PD}_{3}$-complexes. We defer the proof to Chapter 3, where we shall give an extension which applies also to certain $\mathrm{PD}_{3}$-pairs. In $\$ 4$ we prove a weak version, which ignores the role of the fundamental class, and in $\$ 5$ we state the full version. In the next two sections we give Turaev's proofs of his other major results, that a $\mathrm{PD}_{3}$-complex $P$ is a proper connected sum if and only if $\pi_{1}(P)$ is a proper free product, and that an oriented $\mathrm{PD}_{3}$-complex has an essentially unique factorization as a connected sum of indecomposable complexes. Turaev showed also that the finiteness obstruction may be defined in terms of the ideas he introduced. In $\$ 8$ we state his result, and comment briefly on issues related to Whitehead torsion and surgery. The final section describes $\pi_{2}(P)$ as a $\mathbb{Z}\left[\pi_{1}(P)\right]$-module.

\subsection{A low-dimensional simplification}

In dimensions $n<3$ the criterion for a finitely dominated cell complex to be a $\mathrm{PD}_{n}$-complex may be simplified, in that we do not need to know a priori that the chain homotopy equivalence establishing duality is given by cap product with an $n$-cycle. The following proof of this assertion for the case $n=3$ is based on ideas from [147], but has somewhat different hypotheses.

If $E_{*}$ is a chain complex, let $D E_{*}=E^{3-*}$ be the chain complex given by re-indexing the dual cochain complex. If $M$ is a $\mathbb{Z}[\pi]$-module let $e^{i} M=\operatorname{Ext}_{\mathbb{Z}[\pi]}(M ; \mathbb{Z}[\pi])$, for $i \geqslant 0$.

Theorem 2.1. Let $K$ be a connected 3-complex and let $w: \pi=\pi_{1}(K) \rightarrow\{ \pm 1\}$ be a homomorphism. If $C_{*}(K ; \mathbb{Z}[\pi])$ is chain homotopy equivalent to a finite projective $\mathbb{Z}[\pi]$-complex $C_{*}$ such that the chain complexes $D C_{*}=\overline{\operatorname{Hom}_{\mathbb{Z}[\pi]}\left(C_{3-*}, \mathbb{Z}[\pi]\right)}$ and $C_{*}$ are chain homotopy equivalent, then $K$ is a $\mathrm{PD}_{3}$-space. Proof. If $\pi$ is finite then $H_{2}\left(C_{*}\right) \cong H^{1}\left(C^{*}\right)=0$ and $H_{3}\left(C_{*}\right) \cong H^{0}\left(C^{*}\right) \cong \mathbb{Z}$. Therefore $\widetilde{K} \simeq S^{3}$ and so $K$ is a $\mathrm{PD}_{3}$-complex by Lemma 1.3. We may assume henceforth that $\pi$ is infinite.

Let $C_{*} \otimes_{\mathbb{Z}} C_{*}$ have the diagonal left $\pi$-action, and let $\tau(x \otimes y)=(-1)^{p q} y \otimes x$ for all $x \in C_{p}$ and $y \in C_{q}$. Let $\Delta: C_{*} \rightarrow C_{*} \otimes_{\mathbb{Z}} C_{*}$ be a diagonal approximation. Then $\tau \Delta$ is also a diagonal approximation, and so is chain homotopic to $\Delta$. Let $\kappa \in C_{3}$ be a 3-chain such that $1 \otimes \kappa$ is a cycle representing a generator $[K]$ of

$$
H_{3}\left(\mathbb{Z}^{w} \otimes_{\mathbb{Z}[\pi]} C_{*}\right) \cong H_{3}\left(\mathbb{Z}^{w} \otimes_{\mathbb{Z}[\pi]} D C_{*}\right)=H^{0}\left(C^{*} ; \mathbb{Z}^{w}\right) \cong \mathbb{Z},
$$

and let $\Delta(\kappa)=\sum \sum x_{i, j} \otimes y_{3-i, j}$. Cap product with $1 \otimes \kappa$ defines a chain map $\theta_{*}: D C_{*} \rightarrow C_{*}$ by $\theta(\phi)=\sum \phi\left(x_{3-i, j}\right) y_{i, j}$ for all $\phi \in C^{3-i}$. We shall show that $\theta_{*}$ is a chain homotopy equivalence. The 
double dual $D D C_{*}$ is naturally isomorphic to $C_{*}$, and $\theta_{*}$ induces a dual map $D \theta_{*}: D C_{*} \rightarrow D D C_{*}=C_{*}$. The "symmetry" of $\Delta$ with respect to $\tau$ implies that $D \theta_{*}$ and $\theta_{*}$ are chain homotopic, as in [147].

The epimorphism $C^{3} \rightarrow H^{3}\left(C^{*}\right)=H_{0}\left(D C_{*}\right) \cong H_{0}\left(C_{*}\right) \cong \mathbb{Z}$ factors through $\mathbb{Z} \otimes_{\mathbb{Z}[\pi]} C^{3}=\operatorname{Hom}\left(\mathbb{Z}^{w} \otimes_{\mathbb{Z}[\pi]}\right.$ $C_{3}, \mathbb{Z}$ ). Hence there is a $\phi \in C^{3}$ such that $\phi(\kappa)=1$ and so $H_{0}\left(\theta_{*}\right)$ is an isomorphism (since $\Delta$ is a diagonal approximation). Since $\pi$ is infinite, $H^{0}\left(D C^{*}\right)=H^{0}\left(C^{*}\right)=0$. Hence $H_{3}\left(C_{*}\right)=H_{3}\left(D C_{*}\right)=$ $H_{1}\left(C_{*}\right)=H_{1}\left(D C_{*}\right)=0$, and so $H_{1}\left(\theta_{*}\right)$ and $H_{3}\left(\theta_{*}\right)$ are trivially isomorphisms. In particular, the dual $\theta^{*}: C^{*} \rightarrow D C^{*}$ induces an isomorphism $e^{1} H_{0}\left(D C_{*}\right) \cong e^{1} H_{0}\left(C_{*}\right)$, since $H_{0}\left(\theta_{*}\right)$ is an isomorphism. It follows that $H_{2}\left(\theta_{*}\right)=H_{2}\left(D \theta_{*}\right)$ is also an isomorphism, since $H_{2}\left(D C_{*}\right)=H^{1}\left(C^{*}\right)$ and $H_{2}\left(C_{*}\right)=H^{1}\left(D C^{*}\right)$ are naturally isomorphic to $e^{1} H_{0}\left(C_{*}\right)$ and $e^{1} H_{0}\left(D C_{*}\right)$, respectively. Hence $\theta$ is a chain homotopy equivalence, and so $K$ is a $\mathrm{PD}_{3}$-space.

A similar (and easier) result is true for complexes of dimension 1 or 2 . On the other hand, the 1connected space $S^{2} \vee S^{4}$ is not a $\mathrm{PD}_{4}$-complex, although it has a cell structure with just 3 cells, and the cellular chain complex is obviously isomorphic to its linear dual.

\subsection{The Classification Theorem}

The fundamental triple of a $\mathrm{PD}_{3}$-complex $P$ is $\left[\pi_{1}(P), w_{1}(P), c_{P *}[P]\right]$, where $c_{P}: P \rightarrow K\left(\pi_{1}(P), 1\right)$ is the classifying map and $[P]$ is the fundamental class in $H_{3}\left(P ; \mathbb{Z}^{w_{1}(P)}\right)$. There is an obvious notion of isomorphism for such triples. Note, however, that if $P$ and $Q$ are nonorientable $\mathrm{PD}_{n}$-spaces then a map $f: P \rightarrow Q$ such that $f^{*} w_{1}(Q)=w_{1}(P)$ only determines a homomorphism from $H_{n}\left(P ; \mathbb{Z}^{w_{1}(P)}\right)$ to $H_{n}\left(Q ; \mathbb{Z}^{w_{1}(Q)}\right)$ up to sign, as one must choose a lift $f^{+}: P^{+} \rightarrow Q^{+}$. Thus it is only meaningful to specify the sign of $[P]$ if we work with pointed spaces. (See [139] for a thorough discussion of the subtleties here. This issue has no serious consequences for the present work, and it is convenient to keep track of the signs when considering orientable complexes.)

Hendriks showed that this triple is a complete homotopy invariant for such complexes [58]. (For orientable 3-manifolds, this was shown earlier by Swarup [138].)

Lemma 2.2. Let $f: P \rightarrow Q$ be a map between two $\mathrm{PD}_{3}$-complexes. Then $f$ is a homotopy equivalence if and only if $\pi_{1}(f)$ is an isomorphism, $w_{1}(P)=f^{*} w_{1}(Q)$ and $H_{3}\left(f ; \mathbb{Z}^{w_{1}(Q)}\right)$ is an isomorphism.

Proof. The conditions are clearly necessary. Suppose that they hold, and let $\tilde{f}: \widetilde{P} \rightarrow \widetilde{Q}$ be a lift of $f$. If $\pi=\pi_{1}(P)$ is finite then $\widetilde{P}, \widetilde{Q} \simeq S^{3}$, and $\pi_{3}(f)=H_{3}(\tilde{f} ; \mathbb{Z})$ is an isomorphism.

If $\pi$ is infinite then $f$ induces an isomorphism of end modules $E\left(\pi_{1}(Q)\right) \cong E\left(\pi_{1}(P)\right)$. Hence $\pi_{2}(f)=$ $\mathrm{H}_{2}(\tilde{f} ; \mathbb{Z})$ is an isomorphism, by Poincaré duality.

In each case $H_{i}(\tilde{f} ; \mathbb{Z})$ is an isomorphism for all $i \geqslant 0$, and so the lemma follows from the Hurewicz and Whitehead Theorems.

If $P$ and $Q$ are orientable and $P$ has an aspherical summand, $P_{1}$ say, so $P=P_{1} \sharp P_{2}$, then the hypotheses may be simplified [138]. (Let $c: P \rightarrow P_{1}$ collapse the second summand, and let $g: Q \rightarrow P_{1}$ be a map inducing $\pi_{1}(c) \pi_{1}(f)^{-1}$. Then $g f \sim c$, since $P_{1}$ is aspherical, and so $\operatorname{deg} g \operatorname{deg} f=\operatorname{deg} c= \pm 1$. Thus $f$ must have degree \pm 1 .) We shall see in Chapter 4 that $P$ must have such a summand if $\pi$ is not virtually free.

We need some ingredients from obstruction theory. Let $P$ and $Q$ be $\mathrm{PD}_{3}$-complexes such that $P=$ $P_{o} \cup_{g} e^{3}$ and $Q=Q_{o} \cup_{g^{\prime}} e^{3}$, where $P_{o}$ and $Q_{o}$ are homologically 2-dimensional. If $f^{o}: P_{o} \rightarrow Q_{o}$ is a 
map and $f^{[1]}$ is its restriction to the 1 -skeleton $P^{[1]}$ then the primary obstruction to extending $f^{[1]}$ to a map from $P$ to $Q_{o}$ is a class

$$
\mathcal{O}\left(f^{o}\right) \in H^{3}\left(P ;\left(f^{o}\right)^{*} \pi_{2}\left(Q_{o}\right)\right) .
$$

(This obstruction depends only on $f^{[1]}$.) In particular, the primary obstruction to retracting $P$ onto $P_{o}$ is $A(P)=\mathcal{O}\left(\iota_{o}\right)$, where $\iota_{o}: P_{o} \rightarrow P$ is the inclusion. The latter obstruction is represented by the cocycle with value $[g]$ on the 3 -cell $\overline{P \backslash P_{o}}$ and 0 on all other 3 -cells. Let

$$
\mathfrak{A}(P)=A(P) \cap[P] \in H_{0}\left(P ; \mathbb{Z}^{w} \otimes \pi_{2}\left(P_{o}\right)\right) .
$$

Returning to the map $f^{o}: P_{o} \rightarrow Q_{o}$, we have $\mathcal{O}\left(f^{o}\right)=\varphi_{\#} A(P)$, where $\varphi_{\#}$ is the change of coefficients homomorphism. If $f^{[1]}$ can be extended to a map $f: P \rightarrow Q$ then $\mathcal{O}\left(f^{o}\right)=f^{*} A(P)$. Hence

$$
f_{*}\left(\varphi_{\#} \mathfrak{A}(P)\right)=f_{*}\left(\varphi_{\#} A(P) \cap[P]\right)=f_{*}\left(f^{*} A(Q) \cap[P]\right)=A(Q) \cap f_{*}[Q],
$$

and so $f_{*}\left(\varphi_{\#} \mathfrak{A}(P)\right)=d \mathfrak{A}(Q)$, if $f_{*}[P]=d[Q]$.

Suppose conversely that $f_{*}^{[1]}\left(\varphi_{\#} \mathfrak{A}(P)\right)=d \mathfrak{A}(Q)$. Then $\mathcal{O}\left(f^{o}\right)$ is represented by the cocycle with value $d\left[g^{\prime}\right]$ on the 3-cell $\overline{P \backslash P_{o}}$ and 0 on all other 3-cells. Since $g^{\prime}=0 \in \pi_{2}(Q)$, the function $f^{[1]}$ extends to a map $f: P \rightarrow Q$ such that $f_{*}\left(\varphi_{\#} \mathfrak{A}(P)\right)=d \mathfrak{A}(Q)$. By the equations displayed above, this map $f$ must have degree $\pm d$.

We now translate these observations into terms involving the fundamental group.

Theorem 2.3 [58]. Let $P$ and $Q$ be $\mathrm{PD}_{3}$-complexes, and let $\varphi$ be an epimorphism from $\pi=\pi_{1}(P)$ to $\rho=\pi_{1}(Q)$ such that $w_{1}(P)=w_{1}(Q) \circ \varphi$. Let $d \neq 0$ be an integer. Then there is a map $f: P \rightarrow Q$ such that $\pi_{1}(f)=\varphi$ and $f_{*}[P]= \pm d[Q]$ if and only if $\varphi_{*}\left(c_{P *}[P]\right)=c_{Q *}[Q]$.

Proof. The condition is clearly necessary.

Suppose that $\varphi$ satisfies it. We may assume that $P=P_{o} \cup_{g} e^{3}$ and $Q=Q_{o} \cup_{g^{\prime}} e^{3}$, where $P_{o}$ and $Q_{o}$ are homologically 2-dimensional, and $g$ and $g^{\prime}$ are the attaching maps. Let $w=w_{1}(Q)$. There is a map $f: P_{o} \rightarrow Q$ which induces $\varphi$, and there is no obstruction to homotoping $\left.f\right|_{P_{o}}$ to have image in $Q_{o}$.

We may construct an Eilenberg-Mac Lane space $K=K(\pi, 1)$ by adjoining cells of dimension $\geqslant 3$ to $P$. The relative Hurewicz homomorphism in $\widetilde{K}$ projects to give an isomorphism

$$
\operatorname{hwz}: \mathbb{Z}^{w} \otimes_{\mathbb{Z}[\pi]} \pi_{3}\left(K, P_{o}\right) \cong H_{3}\left(K, P_{o} ; \mathbb{Z}^{w}\right) .
$$

The natural homomorphism $e: H_{3}\left(K ; \mathbb{Z}^{w}\right) \rightarrow H_{3}\left(K, P_{o} ; \mathbb{Z}^{w}\right)$ is a monomorphism, since $H_{3}\left(P_{o} ; \mathbb{Z}^{w}\right)=0$, while the connecting homomorphism $\partial: \pi_{3}\left(K, P_{o}\right) \rightarrow \pi_{2}\left(P_{o}\right)$ in the exact sequence of homotopy for $\left(K, P_{o}\right)$ is an isomorphism, since $K$ is aspherical. Hence $h_{P}=(1 \otimes \partial)(\mathrm{hwz})^{-1} e$ is a monomorphism.

We may identify $\mathbb{Z}^{w} \otimes_{\mathbb{Z}[\pi]} \pi_{2}\left(P_{o}\right)$ with $H_{0}\left(P ; \overline{\pi_{2}\left(P_{o}\right)}\right)$. Then

$$
h_{P}\left(c_{P *}[P]\right)=[1 \otimes[g]]=\mathfrak{A}(P) .
$$

Since $h_{P}$ is a monomorphism and is natural with respect to fundamental group homomorphisms compatible with the orientation characters, the result follows.

Taking $d= \pm 1$ gives the following.

Corollary 2.3.1 (Classification Theorem [58]). Two $\mathrm{PD}_{3}$-complexes $P$ and $Q$ are homotopy equivalent if and only if their fundamental triples are isomorphic. 
Turaev gave another proof of the Classification Theorem, related to his proofs of the Realization and Splitting Theorems [147].

\subsection{Homological triple versus algebraic 2-type}

C. B. Thomas gave an alternative set of invariants, based on the Postnikov approach, for orientable 3manifolds [141]. This applies equally well for $\mathrm{PD}_{3}$-complexes. When $\pi$ is finite, the approaches are equivalent, but the connection is not clear when $\pi$ is infinite.

Let $P$ be a $\mathrm{PD}_{3}$-space with fundamental group $\pi$ and orientation character $w$. If $\pi$ is finite then $w=0$ and $\pi_{2}(P)=0$, and $P$ is determined by $k_{2}(P) \in H^{4}(\pi ; \mathbb{Z})$, which is the first nontrivial $k$ invariant. (See Theorem 5.2.) In this case, $k_{2}(P)$ and $c_{P *}[P] \in H_{3}(\pi ; \mathbb{Z})$ are essentially equivalent. The image of the orientation class of $X$ generates $H_{3}(\pi ; \mathbb{Z}) \cong \mathbb{Z} /|\pi| \mathbb{Z}$. The Bockstein homomorphism $\beta: H^{3}(\pi ; \mathbb{Q} / \mathbb{Z}) \rightarrow H^{4}(\pi ; \mathbb{Z})$ is an isomorphism, since $H^{q}(\pi ; \mathbb{Q})=0$ for $q>0$, and the bilinear pairing from $H_{3}(\pi ; \mathbb{Z}) \times H^{4}(\pi ; \mathbb{Z})$ to $\mathbb{Q} / \mathbb{Z}$ given by $(h, c) \mapsto \beta^{-1}(c)(h)$ is nonsingular. Each generator $g$ of $H_{3}(\pi ; \mathbb{Z})$ determines an unique $k_{g} \in H^{4}(\pi ; \mathbb{Z})$ such that $\beta^{-1}\left(k_{g}\right)(g)=\frac{1}{|\pi|} \bmod \mathbb{Z}$. Thomas showed that if $g=c_{X *}[X]$ then $k_{g}=k_{2}(P)$.

Suppose that $\pi$ is infinite. If $N$ is another $\mathrm{PD}_{3}$-space and there is an isomorphism $\theta: v=\pi_{1}(N) \rightarrow \pi$ such that $w_{1}(N)=\theta^{*} w$ then $\pi_{2}(N) \cong \theta^{*} \pi_{2}(P)$ as $\mathbb{Z}[v]$-modules. If moreover $k_{1}(N)=\theta^{*} k_{1}(P)$ (modulo automorphisms of the pair $\left.\left(v, \pi_{2}(N)\right)\right)$ then $P_{2}(N) \simeq P_{2}(P)$. Since we may construct these Postnikov 2-stages by adjoining cells of dimension $\geqslant 4$ it follows that there is a map $f: N \rightarrow P$ such that $\pi_{1}(f)=\theta$ and $\pi_{2}(f)$ is an isomorphism. Since $H_{i}(\tilde{N})=H_{i}(\widetilde{P})=0$ if $i>2$, the map $f$ is a homotopy equivalence, by the Hurewicz and Whitehead Theorems. Thus the homotopy type of $P$ is determined by the triple $\left(\pi, w, k_{1}(P)\right)$. One may ask how $c_{P *}[P]$ and $k_{1}(P)$ determine each other.

In the Realization Theorem the element of $H_{3}\left(\pi ; \mathbb{Z}^{w}\right)$ is used to construct a cell complex $X$ by attaching 2- and 3-cells to the 2-skeleton of $K(\pi, 1)$. (See Chapter 3.) If $C_{*}$ is the cellular chain complex of $\widetilde{X}$ then $k_{1}(X)$ is the class of

$$
0 \rightarrow \pi_{2}(X) \rightarrow C_{2} / \partial C_{3} \rightarrow C_{1} \rightarrow C_{0} \rightarrow \mathbb{Z} \rightarrow 0
$$

in $H^{3}\left(\pi ; \pi_{2}(X)\right)=\operatorname{Ext}_{\mathbb{Z}[\pi]}^{3}\left(\mathbb{Z}, \pi_{2}(X)\right)$.

Conversely, a class $\kappa \in \operatorname{Ext}_{\mathbb{Z}[\pi]}^{3}(\mathbb{Z}, \Pi)$ corresponds to an extension

$$
0 \rightarrow \Pi \rightarrow D_{2} \rightarrow D_{1} \rightarrow D_{0} \rightarrow \mathbb{Z} \rightarrow 0
$$

with $D_{0}$ and $D_{1}$ finitely generated free $\mathbb{Z}[\pi]$-modules. Let $\mathcal{D}_{*}$ be the complex $D_{2} \rightarrow D_{1} \rightarrow D_{0}$, with augmentation $\varepsilon$ to $\mathbb{Z}$. If $\kappa=k_{1}(P)$ for a $\operatorname{PD}_{3}$-complex $P$ then $\mathbb{T o r}_{3}^{\mathbb{Z}[\pi]}\left(\mathbb{Z}^{w}, \mathcal{D}_{*}\right) \cong H_{3}\left(P_{2}(P) ; \mathbb{Z}^{w}\right) \cong \mathbb{Z}$ (where $\mathbb{T}$ or denotes hyperhomology), and the augmentation then determines a class in $H_{3}\left(\pi ; \mathbb{Z}^{w}\right)=$ $\operatorname{Tor}_{3}^{\mathbb{Z}[\pi]}\left(\mathbb{Z}^{w}, \mathbb{Z}\right)$ (up to sign). Can these connections be made more explicit?

\subsection{The Group Realization Theorem}

The characterization of the pairs $(\pi, w)$ realized by $\mathrm{PD}_{3}$-complexes follows from the Realization Theorem of Turaev, which we formulate later (and prove in Chapter 3). We derive it here as a fairly straightforward consequence of the arguments for Theorem 2.1. 
Theorem 2.4 (Group Realization Theorem). Let $\pi$ be a finitely presentable group and $w: \pi \rightarrow\{ \pm 1\}$ a homomorphism. Then there is a finite $\mathrm{PD}_{3}$-complex $P$ with $\pi_{1}(P) \cong \pi$ and $w_{1}(P)=w$ if and only if $[D I(\pi)]=[I(\pi)]$. There is a standard $\mathrm{PD}_{3}$-complex realizing $(\pi, w)$ if and only if $\pi$ has a presentation of deficiency 0 and $D(I(\pi) ; M) \cong I(\pi)$, where $M$ is the associated square Fox-Lyndon presentation matrix for $I(\pi)$.

Proof. Let $P$ be a connected $\mathrm{PD}_{3}$-complex with $\pi_{1}(P) \cong \pi$ and $w_{1}(P)=w$. We may assume that $P$ has a single 0 -cell and finite 2 -skeleton, and that $C_{*}$ and $D C_{*}$ are finitely generated projective $\mathbb{Z}[\pi]$-complexes. Then $C_{0} \cong \mathbb{Z}[\pi]$ and $\operatorname{Coker}\left(\partial_{2}^{C}\right)=\operatorname{Im}\left(\partial_{1}^{C}\right)$ is the augmentation ideal $I(\pi)$. Let $M$ be the matrix for $\partial_{2}^{C}$ with respect to the bases represented by chosen lifts of the cells of $P$. (This is essentially a Fox-Lyndon matrix.) Since $H_{0}\left(C_{*}\right) \cong H_{0}\left(D C_{*}\right) \cong \mathbb{Z}$ and $I(\pi)=\operatorname{Coker}\left(\partial_{2}^{C}\right)$, Schanuel's Lemma implies that

$$
I(\pi) \oplus D C_{0} \cong \operatorname{Coker}\left(\partial_{2}^{D}\right) \oplus C_{0} .
$$

Since $\partial_{2}^{D}$ has matrix $\bar{M}^{\text {tr }}$ it follows that $[D I(\pi)]=[I(\pi)]$.

If $P=P_{o} \cup e^{3}$ is standard then, after collapsing a maximal tree in $P^{[1]}$, if necessary, we may assume also that $P_{o}$ has one 0 -cell. Since $\chi\left(P_{o}\right)=1$, it is then clear that $\pi$ has a presentation of deficiency 0 . Moreover, $D(I(\pi) ; M)$ is the kernel of the epimorphism from $C^{3} \cong \mathbb{Z}[\pi]$ to $H^{3}\left(C^{*}\right) \cong \mathbb{Z}$. This must be equivalent to the augmentation homomorphism, and so $D(I(\pi) ; M) \cong I(\pi)$.

Conversely, let $K$ be the finite 2-complex associated to a presentation for $\pi$, and let $M$ be the corresponding Fox-Lyndon presentation matrix for $I(\pi)$. Suppose first that $D(I(\pi) ; M) \oplus \mathbb{Z}[\pi]^{m} \cong$ $I(\pi) \oplus \mathbb{Z}[\pi]^{n}$. Let $L=K \vee m S^{2}$. Then $\pi_{1}(L) \cong \pi_{1}(K)$ and $\operatorname{Coker}\left(\partial_{2}^{L}\right) \cong I(\pi) \oplus \mathbb{Z}[\pi]^{n}$. Let $D C_{1}=\overline{\operatorname{Hom}_{\mathbb{Z}[\pi]}\left(C_{2}(L ; \mathbb{Z}[\pi]), \mathbb{Z}[\pi]\right)}$ and let $\alpha$ be the composite

$$
D C_{1} \rightarrow D(I(\pi) ; M) \oplus \mathbb{Z}[\pi]^{m} \cong I(\pi) \oplus \mathbb{Z}[\pi]^{n} \rightarrow \mathbb{Z}[\pi]^{n+1} .
$$

Then $\bar{\alpha}^{\operatorname{tr}}: \mathbb{Z}[\pi]^{n+1} \rightarrow C_{2}(L ; \mathbb{Z}[\pi])$ has image in $\pi_{2}(L)=H_{2}(L ; \mathbb{Z}[\pi])$. Let $\left\{e_{1}, \ldots, e_{n+1}\right\}$ be the standard basis of $\mathbb{Z}[\pi]^{n+1}$, and let $f_{i}$ be a map in the homotopy class of $\bar{\alpha}^{\operatorname{tr}}\left(e_{i}\right)$, for $i \leqslant n+1$. Let $P$ be the 3-complex obtained by adjoining 3-cells to $L$ along the $n+1$ maps $\left\{f_{1}, \ldots, f_{n+1}\right\}$. Then $P$ is a finite 3-complex, and $\pi_{1}(P) \cong \pi$.

The arguments of Theorem 2.1 apply to $P$, as we now show. Let $D C_{*}$ be the chain complex obtained by reindexing the dual cochain complex, so $D C_{q}=\overline{\operatorname{Hom}_{\mathbb{Z}[\pi]}\left(C_{3-q}, \mathbb{Z}[\pi]\right)}$. Clearly $H_{0}\left(C_{*}\right)=H_{0}\left(D C_{*}\right)=\mathbb{Z}$. If $\pi$ is finite then $H^{0}\left(C_{*}\right) \cong H^{0}\left(D C^{*}\right) \cong \mathbb{Z}$ and $H_{1}\left(C_{*}\right)=H_{1}\left(D C_{*}\right)=H^{1}\left(C^{*}\right)=H^{1}\left(D C^{*}\right)=0$. Hence $\widetilde{P} \simeq S^{3}$ and so $P$ is a $\mathrm{PD}_{3}$-complex, by Lemma 1.3 . If $\pi$ is infinite then

$$
H_{1}\left(C_{*}\right)=H_{1}\left(D C_{*}\right)=0, \quad H^{1}\left(C^{*}\right) \cong H^{1}\left(C D^{*}\right) \cong E(\pi),
$$

and $H^{0}\left(C^{*}\right)=H^{0}\left(D C^{*}\right)=0$, so $H_{3}\left(C_{*}\right)=H_{3}\left(D C_{*}\right)=0$. Let $x \in C_{3}$ represent a generator of $H_{3}\left(P ; \mathbb{Z}^{w}\right)$. Then cap product with $x$ gives a chain homomorphism $\theta_{*}$ from $D C_{*}$ to $C_{*}$ which induces isomorphisms in degrees 0 and 1 . Hence it induces isomorphisms $H^{1}\left(C^{*}\right) \cong H^{1}\left(D C^{*}\right)$, and so

$$
H_{2}\left(\theta_{*}\right): H_{2}\left(D C_{*}\right)=H^{1}\left(C^{*}\right) \rightarrow H_{2}\left(C_{*}\right)=H^{1}\left(D C^{*}\right)
$$

is an isomorphism. Since $H_{i}\left(\theta_{*}\right)$ is an isomorphism for all $i$ and $C_{*}$ and $D C_{*}$ are projective chain complexes, $\theta_{*}$ is a chain homotopy equivalence. Hence $P$ is a $\mathrm{PD}_{3}$-complex. 
If $\pi$ has a presentation of deficiency 0 and $D(I(\pi) ; M) \cong I(\pi)$, then $m=n=0$ and so the above argument shows that $K \cup e^{3}$ is a standard $\mathrm{PD}_{3}$-complex.

If $D(I(\pi) ; M) \oplus L \cong I(\pi) \oplus N$, where $L$ and $N$ are finitely generated projective $\mathbb{Z}[\pi]$-modules which are not stably isomorphic, we must adjoin infinitely many 2- and 3-cells to get a finitely dominated $\mathrm{PD}_{3}$ complex, as in [147]. This can be further extended to realize groups which are $\mathrm{FP}_{2}$ but not finitely presentable by $\mathrm{PD}_{3}$-spaces.

It can be shown that if $P$ is a finite $\mathrm{PD}_{3}$-space then $I(\pi)$ has a square presentation matrix.

Changing coefficients gives a weaker but useful condition:

Corollary 2.4.1. Let $f: \mathbb{Z}[\pi] \rightarrow R$ be a homomorphism of rings. If $\pi=\pi_{1}(P)$ for some $\mathrm{PD}_{3}$-complex $P$ then

$$
\left(R \otimes_{f} I(\pi)\right) \oplus R \otimes L \cong\left(R \otimes_{f} D(I(\pi) ; M)\right) \oplus R \otimes N
$$

for some projective $R$-modules $L$ and $M$.

We shall use such conditions in Chapters 6 and 7 to exclude some pairs $(\pi, w)$. On the other hand, we shall justify our constructions of new orientable examples by means of Theorem 2.1 .

We emphasize that Theorem 2.4 is only part of Turaev's determination of the triples $[\pi, w, \mu]$ realized by $\mathrm{PD}_{3}$-complexes. We shall need the full Realization Theorem to establish the Splitting Theorem. However, we shall defer the proof to Chapter 3 , where we give a similar result for certain $\mathrm{PD}_{3}$-pairs.

\subsection{Statement of the Realization Theorem of Turaev}

In this section we shall follow [147] closely.

Suppose that $R$ is a ring with an anti-involution $r \mapsto \bar{r}$. Let $C_{*}$ be a projective $R$-chain complex and let $C^{*}$ be the dual cochain complex, with $C^{q}=C_{q}^{\dagger}$. By reversing the indexing, we obtain another chain complex $C^{-*}$. Let $G_{r}\left(C_{*}\right)=C_{r} / \partial C_{r+1}$. If $C_{r}$ is finitely generated let $F^{r}\left(C_{*}\right)=G_{-r}\left(C^{-*}\right)=C^{r} / \partial C^{r-1}$. These formulae clearly give rise to functors from chain complexes to modules.

Lemma 2.5. Let $f, g: C_{*} \rightarrow D_{*}$ be chain homotopic homomorphisms of projective chain complexes $C_{*}$ and $D_{*}$. If $C_{r}$ is finitely generated then $G_{r}(f)$ and $G_{r}(g)$ are projectively homotopic.

Proof. This is a simple consequence of the definitions of homotopy used here, since $G_{r}(f)-G_{r}(g)$ factors through $C_{r}$.

In particular, if $f: C_{*} \rightarrow D_{*}$ is a chain homotopy equivalence of finitely generated projective complexes then $G_{r}(f)$ and $F^{r}(f)$ are projective homotopy equivalences.

Let $C_{*}$ be a free $\mathbb{Z}[\pi]$-chain complex and let $w: \pi \rightarrow \mathbb{Z}^{\times}$be a homomorphism. Tensoring $C_{*}$ over $\mathbb{Z}[\pi]$ with the short exact sequence

$$
0 \rightarrow \overline{I(\pi)} \rightarrow \mathbb{Z}[\pi] \rightarrow \mathbb{Z}^{w} \rightarrow 0
$$

gives a short exact sequence of chain complexes

$$
0 \rightarrow \overline{I(\pi)} C_{*} \rightarrow C_{*} \rightarrow \mathbb{Z}^{w} \otimes_{\mathbb{Z}[\pi]} C_{*} \rightarrow 0 .
$$

Let $\delta_{r}: H_{r+1}\left(\mathbb{Z}^{w} \otimes_{\mathbb{Z}[\pi]} C_{*}\right) \rightarrow H_{r}\left(\overline{I(\pi)} C_{*}\right)$ be the connecting homomorphism. If $c \in \overline{I(\pi)} C_{r}$ is a cycle, evaluation on $c$ determines a homomorphism $\operatorname{ev}_{r}(c): F^{r}\left(C_{*}\right) \rightarrow I(\pi)$, given by $\operatorname{ev}_{r}(c)([\phi])=\overline{\phi(c)}$, for 
all $\phi \in C^{r}$. If $c^{\prime}=c+\bar{\alpha} \partial(e)$ for some $e \in C_{r+1}$ and $\alpha \in I(\pi)$ then $\left(\operatorname{ev}_{r}\left(c^{\prime}\right)-\operatorname{ev}_{r}(c)\right)[\phi]=\overline{\phi(\partial(e))} \alpha$. Thus $\mathrm{ev}_{r}\left(c^{\prime}\right)-\mathrm{ev}_{r}(c)$ factors through right multiplication by $\alpha$ from $\mathbb{Z}[\pi]$ to $I(\pi)$, and so is null-homotopic. Hence $\mathrm{ev}_{r}$ defines an (additive) homomorphism from $H_{r}\left(\overline{I(\pi)} C_{*}\right)$ to $\left[F^{r}\left(C_{*}\right), I(\pi)\right]$, which we shall also call $\mathrm{ev}_{r}$, for simplicity of notation. We set

$$
v_{C_{*}, r}=\mathrm{ev}_{r} \circ \delta_{r}: H_{r+1}\left(\mathbb{Z}^{w} \otimes_{\mathbb{Z}[\pi]} C_{*}\right) \rightarrow\left[F^{r}\left(C_{*}\right), I(\pi)\right] .
$$

If $f: C_{*} \rightarrow D_{*}$ is a chain homotopy equivalence of free $\mathbb{Z}[\pi]$-chain complexes, then $v_{D_{*}, r}\left(f_{*} \xi\right)=v_{C_{*}, r}(\xi)$ for all $\xi \in H_{r+1}\left(\mathbb{Z}^{w} \otimes_{\mathbb{Z}[\pi]} C_{*}\right)$.

Lemma 2.6 [147, Lemma 2.5]. Let $C_{*}$ be a free $\mathbb{Z}[\pi]$-chain complex such that $C_{2}$ is finitely generated and $H_{2}\left(C_{*}\right)=H_{3}\left(C_{*}\right)=0$. Then $v_{C_{*}, 2}: H_{3}\left(\mathbb{Z}^{w} \otimes_{\mathbb{Z}[\pi]} C_{*}\right) \cong\left[F^{2}\left(C_{*}\right), I(\pi)\right]$ is an isomorphism.

Proof. Since $H_{2}\left(C_{*}\right)=H_{3}\left(C_{*}\right)=0$, the connecting homomorphism $\delta_{2}: H_{3}\left(\mathbb{Z}^{w} \otimes_{\mathbb{Z}[\pi]} C_{*}\right) \rightarrow H_{2}\left(\overline{I(\pi)} C_{*}\right)$ is an isomorphism.

Let $g: F^{2}\left(C_{*}\right) \rightarrow I(\pi)$ be a homomorphism. Since $C_{2}$ is finitely generated, there is a canonical isomorphism $C_{2}^{\dagger \dagger} \cong C_{2}$, and so the composite

$$
C^{2} \rightarrow F^{2}\left(C_{*}\right) \stackrel{g}{\longrightarrow} I(\pi) \rightarrow \mathbb{Z}[\pi]
$$

is given by $x \mapsto \overline{x(c)}$, for some $c \in C_{2}$ and all $x \in C^{2}$. Since this composite is 0 on $\partial C^{1}$, we have $\partial(c)=0$, and $c \in \overline{I(\pi)} C_{2}$, since $\overline{x(c)} \in I(\pi)$ for all $x \in C^{2}$. Hence $\operatorname{ev}_{2}[c]=[g]$ in $\left[F^{2}\left(C_{*}\right), I(\pi)\right]$, and so ev 2 is onto.

Suppose now $c \in \overline{I(\pi)} C_{2}$ is a cycle such that $\operatorname{ev}_{2}[c]=0$. Then there are homomorphisms $h: F^{2}\left(C_{*}\right) \rightarrow$ $\mathbb{Z}[\pi]^{m}$ and $\beta: \mathbb{Z}[\pi]^{m} \rightarrow I(\pi)$ such that $\operatorname{ev}_{2}(c)=\beta \circ[h]$. Then there are cycles $c_{1}, \ldots, c_{m} \in C_{2}$ such that the composite $C^{2} \rightarrow F^{2}\left(C_{*}\right) \rightarrow \mathbb{Z}[\pi]^{m}$ is given by $x \mapsto\left(\overline{x\left(c_{1}\right)}, \ldots, \overline{x\left(c_{m}\right)}\right)$. Let $\beta_{i}$ be the image in $I(\pi)$ of the $i$-th standard generator of $\mathbb{Z}[\pi]^{m}$. Then $c=\sum \overline{\beta_{i}} c_{i}$. There are chains $d_{i} \in C_{3}$ such that $\partial d_{i}=c_{i}$, for $i \leqslant m$, since the $c_{i}$ are cycles and $H_{2}\left(C_{*}\right)=0$. Hence $c=\partial\left(\sum \overline{\beta_{i}} d_{i}\right)$, which is in $\partial \overline{I(\pi)} C_{3}$. Thus $\mathrm{ev}_{2}$ is also an isomorphism and so $v_{C_{*}, 2}$ is an isomorphism.

Theorem (Turaev's Realization Theorem [147]). Let $\pi$ be an $\mathrm{FP}_{2}$ group, $w: \pi \rightarrow \mathbb{Z}^{\times}$a homomorphism and $\mu \in H_{3}\left(\pi ; \mathbb{Z}^{w}\right)$. Let $C_{*}$ be a free $\mathbb{Z}[\pi]$-resolution of $\mathbb{Z}$ which is finitely generated in degrees $\leqslant 2$. Then there is a $\mathrm{PD}_{3}$-space realizing the triple $[\pi, w, \mu]$ if and only if $v_{C_{*}, 2}(\mu)$ is a projective homotopy equivalence.

We shall give Bleile's extension of this result to a Realization Theorem for certain $\mathrm{PD}_{3}$-pairs in Chapter 3.

The Classification and Realization Theorems have a slightly more precise common formulation. In [4], Baues and Bleile define three categories:

(1) $\mathrm{PD}_{+}^{3} / \sim$ : homotopy types of $\mathrm{PD}_{3}$-complexes (with pointed universal covers) and degree-1 maps;

(2) $\mathrm{PD}_{*+}^{3} / \sim$ : chain homotopy types of " $\mathrm{PD}_{3}$-chain complexes" and degree-1 chain morphisms; and

(3) $\operatorname{Trp}_{+v}^{3}$ : Hendriks triples and epimorphisms of groups which are compatible with the other data.

They observed that the natural invariants define functors

$$
\mathrm{PD}_{+}^{3} / \sim \rightarrow \mathrm{PD}_{*+}^{3} / \sim \rightarrow \operatorname{Trp}_{+v}^{3}
$$


which are full (surjective on morphisms) and reflect isomorphisms (a morphism is an isomorphism if its image is an isomorphism).

The homotopy type of a higher dimensional $\mathrm{PD}_{n}$-complex $X$ is determined by the triple

$$
\left(P_{n-2}(X), w, f_{X *}[X]\right),
$$

where $f_{X}: X \rightarrow P_{n-2}(X)$ is the Postnikov $(n-2)$-stage and $w=w_{1}(X)$ [4]. If $\tilde{X}$ is $(n-2)$-connected then $P_{n-2}(X) \simeq K(\pi, 1)$, so this triple is a direct analogue of Hendriks' invariant.

\subsection{Connected sum decompositions}

It is a well known consequence of the Sphere Theorem that every closed orientable 3-manifold is a connected sum of indecomposable factors, which are either aspherical or have fundamental group $\mathbb{Z}$ or a finite group. In his foundational 1967 paper, Wall asked whether $\mathrm{PD}_{3}$-complexes behave like 3-manifolds with regards to connected sum [150]. Consider the following conditions for a $\mathrm{PD}_{3}$-complex $P$ :

(1) $P$ is a proper connected sum (the sum of two $\mathrm{PD}_{3}$-complexes neither of which is 1-connected).

(2) $\pi=\pi_{1}(P)$ is a proper free product.

(3) Either $e(\pi)=\infty$ or $\pi \cong \mathbb{Z} / 2 \mathbb{Z} * \mathbb{Z} / 2 \mathbb{Z}$.

Clearly $(1) \Rightarrow(2) \Rightarrow(3)$, and these conditions are equivalent if $P$ is a closed, orientable 3-manifold. Wall asked whether either of these implications could be reversed for general $\mathrm{PD}_{3}$-complexes, and observed that the implication (2) $\Rightarrow$ (1) seemed more likely [150]. Turaev used his Realization Theorem to show that $(1) \Leftrightarrow(2)$ (the "Splitting Theorem"), while Crisp showed that every indecomposable orientable $\mathrm{PD}_{3}$ complex is either aspherical or its fundamental group is the fundamental group of a finite graph of finite groups [22]. (We shall give his proof in Chapter 4.) However, the group may have infinitely many ends, in contrast to the situation for 3-manifolds. We shall give an explicit example with $\pi \cong S_{3} * \mathbb{Z} / 2 \mathbb{Z} S_{3}$ in Chapter 6.

We shall say that a $\mathrm{PD}_{3}$-complex is indecomposable if it is not a proper connected sum.

Theorem 2.7 (Splitting Theorem [147]). A $\mathrm{PD}_{3}$-complex $P$ with fundamental triple $[\pi, w, \mu]$ is a proper connected sum $P_{1} \sharp P_{2}$ if and only if $\pi$ is a proper free product.

Proof. The condition is clearly necessary. Suppose that it holds. Then $\pi=\pi_{1}(P) \cong G_{1} * G_{2}$ where $G_{1}$ and $G_{2}$ are nontrivial groups. Let $\iota_{i}: G_{i} \rightarrow \pi$ be the inclusion, and let $\rho_{i}: \pi \rightarrow G_{i}$ be the retraction, for $i=1,2$. Let $w_{i}=\left.w\right|_{G_{i}}$, for $i=1,2$. Then $w=w_{1} \circ \rho_{1}+w_{2} \circ \rho_{2}$, and

$$
H_{3}\left(\pi ; \mathbb{Z}^{w}\right) \cong H_{3}\left(G_{1} ; \mathbb{Z}^{w_{1}}\right) \oplus H_{3}\left(G_{2} ; \mathbb{Z}^{w_{2}}\right) .
$$

Let $\mu_{i}=H_{3}\left(\rho_{i}\right)(\mu) \in H_{3}\left(G_{i} ; \mathbb{Z}^{w_{i}}\right)$, for $i=1,2$. Then $\mu=\mu_{1}+\mu_{2}$. We shall construct $\mathrm{PD}_{3}$-complexes $P_{1}$ and $P_{2}$ with fundamental triples $\left[G_{i}, w_{i}, \mu_{i}\right]$, for $i=1,2$.

Since $\pi$ is finitely presentable, so are the factors $G_{1}$ and $G_{2}$. Hence there are $K\left(G_{i}, 1\right)$-complexes $K_{i}$, with one 0 -cell and finite 2-skeleton, for $i=1,2$, and $K=K_{1} \vee K_{2}$ is a $K(\pi, 1)$-complex. Let $C(i)_{*}=C_{*}\left(\widetilde{K}_{i}\right)$, for $i=1,2$, and $C_{*}=C_{*}(\widetilde{K})$. Let $\alpha^{i}$ be the change of coefficients functor $\mathbb{Z}[\pi] \otimes_{\mathbb{Z}\left[G_{i}\right]}-$, and let $\beta^{i}$ be the left inverse induced by the projection of $\pi$ onto $G_{i}$, for $i=1,2$. Then

$$
C_{q}=\alpha^{1} C(1)_{q} \oplus \alpha^{2} C(2)_{q}
$$


when $q>0$. Hence $F^{2}\left(C_{*}\right)=\alpha^{1} F^{2}\left(C(1)_{*}\right) \oplus \alpha^{2} F^{2}\left(C(2)_{*}\right)$. Similarly, $I(\pi)=\alpha^{1} I\left(G_{1}\right) \oplus \alpha^{2} I\left(G_{2}\right)$.

Let $f_{i}: F^{2}\left(C(i)_{*}\right) \rightarrow I\left(G_{i}\right)$ be a representative of $v_{C(i)_{*}}\left(\mu_{i}\right)$, for $i=1,2$. Then $v_{C_{*}}(\mu)$ is represented by the homomorphism

$$
\alpha^{1} f_{1} \oplus \alpha^{2} f_{2}: \alpha^{1} F^{2}\left(C(1)_{*}\right) \oplus \alpha^{2} F^{2}\left(C(2)_{*}\right) \rightarrow \alpha^{1} I\left(G_{1}\right) \oplus \alpha^{2} I\left(G_{2}\right) .
$$

We shall show that each $f_{i}$ is a projective homotopy equivalence. Since $v_{C_{*}}(\mu)$ is a projective homotopy equivalence there are finitely generated projective modules $L$ and $M$ and a homomorphism $j$ such that the following diagram commutes

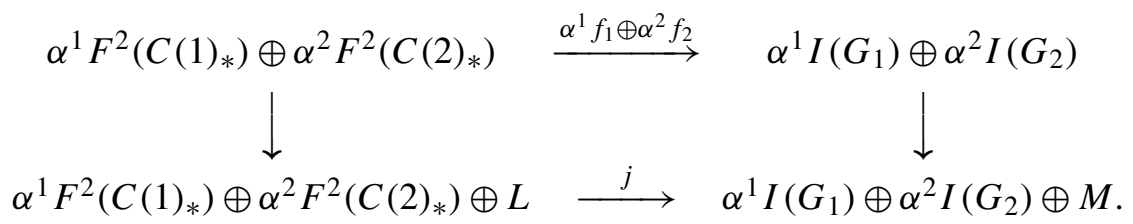

We apply the functor $\beta^{i}$. Clearly $\beta^{i} \circ \alpha^{i}=\mathrm{id}$ and $\beta^{i} L$ and $\beta^{i} M$ are finitely generated projective $\mathbb{Z}\left[G_{i}\right]-$ modules. Applying $\beta^{i}$ to a finitely generated $\mathbb{Z}\left[G_{3-i}\right]$-module gives a module of the form $\mathbb{Z}\left[G_{i}\right] \otimes A$, where $A$ is a finitely generated abelian group. Hence $\mathbb{Z}\left[G_{i}\right] \otimes A$ is the direct sum of a finitely generated free $\mathbb{Z}\left[G_{i}\right]$-module with a $\mathbb{Z}$-torsion module of finite exponent. In particular, when $i=1$ we obtain a diagram

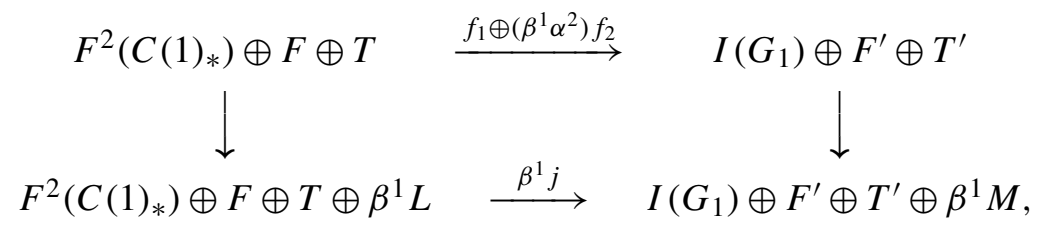

where $F$ and $F^{\prime}$ are free $\mathbb{Z}\left[G_{1}\right]$-modules and $T$ and $T^{\prime}$ have finite exponent. It follows from the commutativity of the diagram and the nature of the homomorphism $f_{1} \oplus\left(\beta^{1} \alpha^{2}\right) f_{2}$ that $\beta^{1} j\left(F^{2}\left(C(1)_{*}\right) \leqslant\right.$ $I\left(G_{1}\right) \oplus \beta^{1} M$. Since $\beta^{1} j$ is an isomorphism and $I\left(G_{1}\right)$ and $\beta^{1} M$ are torsion-free, so is $F^{2}\left(C(1)_{*}\right)$. Therefore we may factor out the torsion submodules to get a simpler commuting diagram

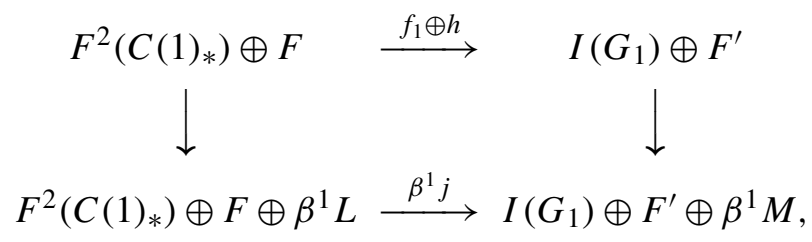

where $h$ is a homomorphism of free modules. By the commutativity of this diagram, $f_{1}$ is the composite

$$
F^{2}\left(C(1)_{*}\right) \rightarrow F^{2}\left(C(1)_{*}\right) \oplus F \oplus \beta^{1} L \cong I\left(G_{1}\right) \oplus F^{\prime} \oplus \beta^{1} M \rightarrow I\left(G_{1}\right),
$$

where the left- and right-hand maps are the obvious inclusion and projection, respectively. Hence $f_{1}$ is a projective homotopy equivalence, and so $\left[G_{1}, w_{1}, \mu_{1}\right]$ is the fundamental triple of a $\mathrm{PD}_{3}$-complex $Q_{1}$, by the Realization Theorem. Similarly, $\left[G_{2}, w_{2}, \mu_{2}\right]$ is the fundamental triple of a $\mathrm{PD}_{3}$-complex $Q_{2}$. Since the fundamental triple of the sum $Q_{1} \sharp Q_{2}$ is $\left[G_{1} * G_{2}, w_{1} \rho_{1}+w_{2} \rho_{2}, \mu_{1}+\mu_{2}\right]$, we may conclude that $P \simeq Q_{1} \sharp Q_{2}$. 
The analysis of indecomposable $\mathrm{PD}_{3}$-complexes $P$ with $\pi=\pi_{1}(P)$ (in Chapter 4) relies on the following observations:

(1) $\pi_{2}(P) \cong \overline{E(\pi)}$, by Poincaré duality.

(2) $E(\pi)$ has Chiswell-Mayer-Vietoris presentations.

(3) The action of $\pi$ on $\pi_{2}(P)$ has the property stated in Lemma 4.6.

(4) If an orientable $\mathrm{PD}_{3}$-complex $P$ is indecomposable, either it is aspherical or $\pi_{1}(P)$ is virtually free.

This last was proved by Crisp through an ingenious combinatorial argument, and is a substantial partial answer to the second part of Wall's question.

The arguments of Turaev and Crisp extend to $\mathrm{PD}_{3}$-spaces in a straightforward manner. In particular, they imply that if $P$ is a $\mathrm{PD}_{3}$-space then $\pi=\pi_{1}(P)$ is virtually torsion-free. However, there is an indecomposable orientable $\mathrm{PD}_{3}$-complex with $\pi \cong S_{3} * \mathbb{Z} / 2 \mathbb{Z} S_{3} \cong F(2) \rtimes S_{3}$ whose double cover is homotopy equivalent to $L(3,1) \sharp L(3,1)$. "Most" indecomposable $\mathrm{PD}_{3}$-complexes with $\pi$ virtually free have double covers which are homotopy equivalent to connected sums of $\mathbb{S}^{3}$-manifolds. (See Chapter 6.)

The situation is somewhat different in the nonorientable case. If a nonorientable $\mathrm{PD}_{3}$-complex $P$ is indecomposable then either $P \simeq R P^{2} \times S^{1}$ or $S^{2} \widetilde{\times} S^{1}$, or $\pi \cong \pi \mathcal{G}$, where each vertex group of $(\mathcal{G}, \Gamma)$ has one end, and each edge group has order 2. (See Chapter 7.) The orientation-preserving subgroups of the vertex groups are then $\mathrm{PD}_{3}$-groups. Such 3-manifolds are unions of quotients of punctured aspherical 3-manifolds by free involutions. However, it is not known whether the vertices of $\Gamma$ must all have even valence, nor whether a $\mathrm{PD}_{3}$-complex with orientation cover homotopy equivalent to a 3-manifold must be homotopy equivalent to a 3-manifold.

The above arguments apply with little change to the study of $\mathrm{PD}_{n}$-complexes with $(n-2)$-connected universal cover. When $n$ is odd, the results are similar. However when $n$ is even it is not known whether the group must be virtually torsion-free. On the other hand, if the group is indecomposable, virtually free and has no dihedral subgroups of order $>2$, then either it has order $\leqslant 2$ or it has two ends and its maximal finite subgroups have cohomology of period dividing $n$. (See [13].)

\subsection{Uniqueness of factorization}

The Unique Factorization Theorem for orientable $\mathrm{PD}_{3}$-complexes follows easily from the Classification and Splitting Theorems.

Theorem 2.8 (Unique Factorization Theorem [147]). Let $\left\{P_{i}: i \in I\right\}$ and $\left\{Q_{j}: j \in J\right\}$ be finite sets of indecomposable orientable $\mathrm{PD}_{3}$-complexes such that $P=\sharp_{i \in I} P_{i} \simeq Q=\sharp_{j \in J} Q_{j}$. Then there is a bijection $f: I \rightarrow J$ such that $P_{i} \simeq Q_{f(i)}$, for all $i \in I$.

Proof. Suppose that $h$ is a homotopy equivalence. Since the groups $\rho_{i}=\pi_{1}\left(P_{i}\right)$ and $\sigma_{j}=\pi_{1}\left(Q_{j}\right)$ are indecomposable, by the Splitting Theorem, and since $\pi_{1}(P)=*_{i \in I} \rho_{i}$ and $\pi_{1}(Q)=*_{j \in J} \sigma_{j}$, the images under $h_{*}=\pi_{1}(h)$ of the non-free factors of $\pi_{1}(P)$ are conjugate to factors of $\pi_{1}(Q)$, and the numbers of free factors in $\pi_{1}(P)$ and $\pi_{1}(Q)$ are the same, by the Kurosh Subgroup Theorem for free products. Thus we may assume that $I=J=\{1, \ldots, m\}$ and that there is a $k \leqslant m$ such that $h_{*}\left(\rho_{i}\right)=a_{i}\left(\sigma_{i}\right) a_{i}^{-1}$, for some $a_{i} \in \pi_{1}(Q)$ and all $i \leqslant k$, while $\rho_{i} \cong \sigma_{i} \cong \mathbb{Z}$ if $i>k$. 
Let $h_{i}: \rho_{i} \rightarrow \sigma_{i}$ be the homomorphism defined by $h_{i}(x)=a_{i}^{-1} h_{*}(x) a_{i}$, for $i \leqslant k$. The isomorphism

$$
H_{3}\left(h_{*}\right): H_{3}\left(\pi_{1}(P)\right)=\bigoplus_{i \leqslant k} H_{3}\left(\rho_{i}\right) \cong H_{3}\left(\pi_{1}(Q)\right)=\bigoplus_{i \leqslant k} H_{3}\left(\sigma_{i}\right)
$$

restricts to isomorphisms of corresponding summands, since inner automorphisms induce the identity on (co)homology. Since $\mu_{P}=\sum \mu_{P_{i}}$ and $\mu_{Q}=\sum \mu_{Q_{j}}$ and $H_{3}\left(h_{*}\right)$ preserves the direct sum decomposition, we have $H_{3}\left(h_{i}\right)\left(\mu_{P_{i}}\right)=\mu_{Q_{i}}$, for $i \leqslant k$. Hence $P_{i} \simeq Q_{i}$, for $i \leqslant k$. Since $P_{i} \simeq Q_{i} \simeq S^{2} \times S^{1}$ if $i>k$, the theorem is proven.

The situation is slightly more complicated in the nonorientable case, but the result is familiar from the case of manifolds. It is well-known that if $M$ is a nonorientable closed $n$-manifold and we attach a 1handle to get the sum $M \sharp\left(S^{n-1} \times S^{1}\right)$ then we may isotope the attaching map to obtain $M \sharp\left(S^{n-1} \widetilde{\times} S^{1}\right)$. In our context it is not clear how to justify such an argument, but it is easy to see that if $P$ is a nonorientable $\mathrm{PD}_{3}$-complex then the fundamental triples of $P \sharp\left(S^{2} \times S^{1}\right)$ and $P \sharp\left(S^{2} \widetilde{\times} S^{1}\right)$ are isomorphic. The statement of the factorization theorem is then as follows. The proof is similar, except that we must consider also the orientation characters.

Theorem [147]. Let $P$ be a nonorientable PD-complex. Then $P$ has an essentially unique factorization as a sum $\sharp_{i \in I} P_{i}$, where $P_{i}$ is indecomposable and $P_{i} \not S^{2} \times S^{1}$, for all $i$.

\subsection{The finiteness obstruction}

The obstruction to a $\mathrm{PD}_{3}$-complex being homotopy finite may be determined from its fundamental triple. If $P$ is a $\mathrm{PD}_{n}$-complex then $C_{*}(P ; \mathbb{Z}[\pi])$ is chain homotopy equivalent to a finite projective $\mathbb{Z}[\pi]$-complex, $E_{*}$ say. Then $P$ is homotopy equivalent to a finite complex if and only if the finiteness obstruction $\sigma(P)=\sum(-1)^{i}\left[E_{i}\right]$ in $\widetilde{K}_{0}(\mathbb{Z}[\pi])$ is $0[148]$. Let $*$ be the involution of $\widetilde{K}_{0}(\mathbb{Z}[\pi])$ defined by $[P] \mapsto\left[P^{\dagger}\right]$. Then $\sigma(P)^{*}=(-1)^{n} \sigma(P)$, by [150, Theorem 1.3].

Let $\Phi:\left[F^{2}\left(C_{*}\right), I(\pi)\right] \rightarrow \widetilde{K}_{0}(\mathbb{Z}[\pi])$ be the homomorphism defined in the lemma of 1.3 . Then Turaev showed:

Theorem [147, Theorem 6]. Let $[\pi, w, \mu]$ be the fundamental triple of a $\mathrm{PD}_{3}$-complex, and let $C_{*}$ be a finite free $\mathbb{Z}[\pi]$-chain complex such that $H_{0}\left(C_{*}\right) \cong \mathbb{Z}$ and $H_{1}\left(C_{*}\right)=0$. Then the finiteness obstruction for $P$ is $\Phi\left(v_{C_{*}, 2}(\mu)\right)^{*}$.

We mention briefly two issues that shall play no further role in this book.

It is well known that there are lens spaces which homotopy equivalent but not simple homotopy equivalent, and thus not homeomorphic. If $P$ is a finite $\mathrm{PD}_{3}$-complex with fundamental group $\pi$, which elements of $\mathrm{Wh}(\pi)$ are the torsions of self-homotopy equivalences? The torsion $\tau(X)$ of the duality chain homotopy equivalence of a non-simple finite $\mathrm{PD}_{n}$-complex also satisfies a duality condition $\tau(P)^{*}=$ $(-1)^{n} \tau(P)$ [118]. Which elements of $\mathrm{Wh}(\pi)$ are realized as torsions of finite $\mathrm{PD}_{3}$-complexes? It is widely expected that $\mathrm{Wh}(\pi)$ should be 0 if $\pi$ is torsion-free, so these questions may only be of interest when $\pi$ is virtually free.

The Spivak normal fibration of any $\mathrm{PD}_{3}$-complex has a linear reduction. It was realized early in the development of surgery that some of the theory remains valid in low dimensions, provided the notion of structure set is modified. Following [86], we approximate $\mathrm{PD}_{3}$-complexes $P$ by maps $f: M \rightarrow P$ with domain a closed 3-manifold and which induce isomorphisms in homology with coefficients $\mathbb{Z}[\pi]$, where 
$\pi=\pi_{1}(P)$. We require also that these maps have torsion 0 in $\mathrm{Wh}(\pi)$. Two such maps $f: M \rightarrow P$ and $f^{\prime}: M^{\prime} \rightarrow P$ are equivalent if there is a normal bordism $F: W \rightarrow P \times[0,1]$, a $\mathbb{R}^{n}$-bundle $\zeta$ over $P \times[0,1]$ and a bundle map $b: v_{W} \rightarrow F^{*} \zeta$ with surgery obstruction 0 in $L_{4}(\pi, w)$. Let $\overline{\mathcal{S}}^{\mathrm{TOP}}(P)$ be the set of equivalence classes of such maps. Then there is an exact sequence

$$
\overline{\mathcal{S}}^{\mathrm{TOP}}(P) \stackrel{N_{x}}{\longrightarrow}[P, G / \mathrm{TOP}] \stackrel{\theta_{x}}{\longrightarrow} L_{3}(\pi, w) .
$$

If $\pi$ is "good" in the sense of [FQ] (for instance, if it is finite) then we may require that $W$ be a TOP $s$-cobordism in the equivalence relation defining the structure set.

\section{9. $\pi_{2}$ as a $\mathbb{Z}[\pi]$-module}

If $P$ is an orientable 3-manifold which is the connected sum of a 3-manifold whose fundamental group is free of rank $r$ with $s \geqslant 1$ aspherical 3-manifolds, then $\pi_{2}(P)$ is a finitely generated free $\mathbb{Z}[\pi]$-module of rank $r+s-1$ [137]. We shall give a direct homological argument that applies for $\mathrm{PD}_{3}$-spaces with torsionfree fundamental group, and we shall also compute $H^{2}\left(P ; \pi_{2}(P)\right)$ for such spaces. (This cohomology group contains the primary obstruction to homotoping a self map $f$ of $P$ such that $\pi_{1}(f)=\mathrm{id}_{\pi}$ to the identity [59].)

Let $P$ be a $\mathrm{PD}_{3}$-space with fundamental group $\pi$, and let $w=w_{1}(P)$. The Hurewicz Theorem, Poincaré duality and a choice of orientation together determine an isomorphism $\pi_{2}(P) \cong \overline{E(\pi)}$. In particular, $\pi_{2}(P)=0$ if and only if $\pi$ is finite or has one end.

Theorem 2.9. Let $P$ be a $\mathrm{PD}_{3}$-space such that $\pi=\pi_{1}(P)$ is nontrivial and torsion-free, and let $w=$ $w_{1}(P)$.

(1) If $\pi$ is a free group, the $\mathbb{Z}[\pi]$-module $\pi_{2}(P)$ is finitely generated and of projective dimension 1 and $H^{2}\left(P ; \pi_{2}(P)\right)$ is infinite cyclic.

(2) ilf $\pi$ is not free, then $\mathrm{cd} \pi=3$, the $\mathbb{Z}[\pi]$-module $\pi_{2}(P)$ is finitely generated and free, $H_{3}\left(c_{P} ; \mathbb{Z}^{w}\right)$ is a monomorphism and $H^{2}\left(P ; \pi_{2}(P)\right)=0$.

(3) $P$ is homotopy equivalent to a finite $\mathrm{PD}_{3}$-complex if and only if $\pi$ is finitely presentable and finite free $(\mathrm{FF})$.

Proof. Poincaré duality with coefficients $\pi_{2}(P)$ gives an isomorphism

$$
H^{2}\left(P ; \pi_{2}(P)\right) \cong H_{1}(P ; E(\pi))=H_{1}(\pi ; E(\pi)) .
$$

Since $\pi$ is $\mathrm{FP}_{2}$ we have $\pi \cong \pi \mathcal{G}$, where $\mathcal{G}$ is a finite graph of groups with vertex groups finite or one-ended and edge groups finite, and so Theorem 1.2 applies.

If $\pi$ is free of rank $r>0$ we may assume there is one vertex, with trivial vertex group, and $r$ edges. The right $\mathbb{Z}[\pi]$-module $E(\pi)=H^{1}(\pi ; \mathbb{Z}[\pi])$ then has a presentation

$$
0 \rightarrow \mathbb{Z}[\pi] \rightarrow \mathbb{Z}[\pi]^{r} \rightarrow E(\pi) \rightarrow 0 .
$$

On applying the functor $-\otimes_{\mathbb{Z}[\pi]} \mathbb{Z}$, the left-hand homomorphism becomes the trivial homomorphism from $\mathbb{Z} \rightarrow \mathbb{Z}^{r}$. Hence

$$
H^{2}\left(P ; \pi_{2}(P)\right) \cong H_{1}(\pi ; E(\pi))=\operatorname{Tor}_{1}^{\mathbb{Z}[\pi]}(E(\pi), \mathbb{Z}) \cong \mathbb{Z}
$$


by the exact sequence of Tor. Moreover $\pi_{2}(P)$ has projective dimension 1 . As $\pi$ is finitely presentable and projective $\mathbb{Z}[F(r)]$-modules are free [3], $P$ is homotopy equivalent to a finite $\mathrm{PD}_{3}$-complex.

If $\pi$ is torsion-free but not free then we may assume that the vertex groups are finitely generated and have one end, and that the edge groups are trivial. Hence $E(\pi)$ is a free right $\mathbb{Z}[\pi]$-module with basis corresponding to the edges of $\mathcal{G}$, and so $H^{2}\left(P ; \pi_{2}(P)\right)=0$. We may assume that $P$ is 3-dimensional and $C_{*}(P ; \mathbb{Z}[\pi])$ is chain homotopy equivalent to a finitely generated projective $\mathbb{Z}[\pi]$-complex $C_{*}$, where $C_{i}$ is free if $i \leqslant 2$ and is 0 if $i>3$. Let $Z_{2}$ be the module of 2-cycles. Then the sequences

$$
0 \rightarrow Z_{2} \rightarrow C_{2} \rightarrow C_{1} \rightarrow C_{0} \rightarrow \mathbb{Z} \rightarrow 0
$$

and

$$
0 \rightarrow C_{3} \rightarrow Z_{2} \rightarrow \pi_{2}(P) \rightarrow 0
$$

are exact, since $H_{3}(P ; \mathbb{Z}[\pi])=H_{3}(\widetilde{P} ; \mathbb{Z})=0$. Attaching 3 -cells to $P$ along a basis for $\pi_{2}(P)$ gives an aspherical 3-dimensional complex $K$ with fundamental group $\pi$. The inclusion of $P$ into $K$ may be identified with $c_{P}$, and clearly induces monomorphisms $H_{3}(P ; A) \rightarrow H_{3}(\pi ; A)$ for any coefficient module $A$. Hence $\mathrm{cd} \pi=3$.

If $\pi$ is FF there is a finite free resolution

$$
0 \rightarrow D_{3} \rightarrow D_{2} \rightarrow D_{1} \rightarrow D_{0} \rightarrow \mathbb{Z} \rightarrow 0 .
$$

Therefore $Z_{2}$ is finitely generated and stably free, by Schanuel's Lemma. Since $\pi_{2}(P)$ is free, we have $Z_{2} \cong \pi_{2}(P) \oplus C_{3}$, and so $C_{3}$ is also stably free. Hence if moreover $\pi$ is finitely presentable then $P$ is homotopy equivalent to a finite $\mathrm{PD}_{3}$-complex. The converse is clear, by the above construction of $K(\pi, 1) \simeq K$.

The Splitting Theorem may be used show that if $\pi \cong F(r)$ then $P \simeq \sharp^{r}\left(S^{2} \times S^{1}\right)$ or $\sharp^{r}\left(S^{2} \widetilde{\times} S^{1}\right)$. This can be seen more directly. (Suppose that $P \simeq P_{o} \cup_{\phi} e^{3}$, where $P_{o}$ is homologically 2-dimensional. Schanuel's Lemma may be used to show that $P_{o} \simeq \bigvee^{r} S^{1} \vee \bigvee^{r} S^{2}$. Hence $\pi_{2}\left(P_{o}\right)$ is a free $\mathbb{Z}[F(r)]$ module of rank $r$. Changes of base via automorphisms of $F(r)$ and $\pi_{2}\left(P_{o}\right)$ then show that $P$ is standard. Compare [66].)

We may relax the condition that $\pi$ be torsion-free.

Corollary 2.9.1. If $P$ is a $\mathrm{PD}_{3}$-space such that $\pi=\pi_{1}(P)$ is virtually torsion-free then the $\mathbb{Z}[\pi]$-module $\pi_{2}(P)$ is finitely presentable. Moreover, $H^{2}\left(P ; \pi_{2}(P)\right)$ is a finitely generated abelian group of rank 1 if $\pi$ is infinite and virtually free, and is finite otherwise. If $\pi$ is infinite but not torsion-free, the projective dimension of $\pi_{2}(P)$ is infinite.

Proof. The first two assertions follow from the theorem, since they are stable under passage to finite overgroups. If $\pi$ is infinite and $\pi_{2}(P)$ has finite projective dimension then so does $Z_{2}$, and so cd $\pi<\infty$, and then $\pi$ is torsion-free.

We shall see that $\pi$ is always virtually torsion-free.

There is a slightly stronger result in the 3-manifold case. Hendriks and Laudenbach showed that if $V$ is a $P^{2}$-irreducible closed 3-manifold then $H^{2}\left(V ; \pi_{2}(V)\right) \cong \mathbb{Z}$ if $\pi_{1}(V)$ is virtually free, and is 0 otherwise. They used this to show that a based self-map $h$ of $V$ such that $\pi_{1}(h)=\mathrm{id}_{\pi}$ and $H_{3}(h)=1$ is homotopic to $\mathrm{id}_{V}$ [59]. 



\section{CHAPTER 3}

\section{The relative case}

In this chapter, which is based on work of Turaev, Bleile and Crisp [12; 23; 147], we shall give partial extensions of the Classification, Realization and Splitting Theorems to certain $\mathrm{PD}_{3}$-pairs. The fundamental triple has a straightforward extension to all $\mathrm{PD}_{3}$-pairs, but the Classification Theorem has only been proven for pairs with aspherical boundary components. We shall state this without proof in $\S 1$. In $\S 2$ we give Bleile's extension of the Realization Theorem to pairs which have $\pi_{1}$-injective, aspherical boundary components. There are two notions of sum in the relative case: interior connected sum and boundary connected sum. We state the corresponding decomposition theorems in $\$ 3$ (again without proof). In so far as these rely upon the Realization Theorem, the same constraints apply to the boundaries. In $\S 4$ we give Crisp's proof of his Algebraic Loop Theorem, and in $\$ 5$ we consider $\mathrm{PD}_{3}$-pairs with free fundamental group. This is the simplest case in which $\pi_{1}$-injectivity usually fails.

\subsection{The basic invariants}

Let $(P, \partial P)$ be a $\mathrm{PD}_{3}$-pair with $\partial P=\coprod_{i \in I} Y_{i}$. Since every $\mathrm{PD}_{2}$-complex is homotopy equivalent to a closed surface, adjoining the mapping cylinders of homotopy equivalences with domains closed surfaces along each boundary component gives an equivalent pair with boundary a 2-manifold, which has a collared neighbourhood. We shall assume henceforth that this is the case for all $\mathrm{PD}_{3}$-pairs considered.

The pair $(P, \partial P)$ is reduced if no component of $\partial P$ is 1-connected. Let $\kappa_{i}: S_{i}=\pi_{1}\left(Y_{i}\right) \rightarrow \pi=\pi_{1}(P)$ be the homomorphism induced by the inclusion of the $i$-th boundary component. (These homomorphisms depend on the choice of paths connecting basepoints, and so are only well-defined up to conjugacy in $\pi$.) The family $\left\{\kappa_{i}\right\}$ is the peripheral system of the pair, and is $\pi_{1}$-injective if the $\kappa_{i}$ are all monomorphisms. (Thus the components of $\partial \widetilde{P}$ are 1-connected.)

Lemma 3.1. Let $(P, \partial P)$ be a $\mathrm{PD}_{3}$-pair with $\partial P \neq \varnothing$. Then:

(1) $\chi(P)=2 \chi(\partial P)$.

(2) If a boundary component $Y$ is aspherical then the preimages of $Y$ in $\partial \widetilde{P}$ are open surfaces.

(3) If, moreover, $P$ is orientable then $\beta_{1}(Y) \leqslant 2 \operatorname{rank}(B)$, where $B$ is the image of $H_{1}(Y ; \mathbb{Z})$ in $H_{1}(P ; \mathbb{Z})$.

(4) If all the components of $\partial P$ are aspherical then the differential $\partial_{2}: C_{2}(\partial \widetilde{P}) \rightarrow C_{1}(\partial \widetilde{P})$ is injective.

(5) $H^{3}(P ; \mathcal{M})=0$ for any left $\mathbb{Z}[\pi]$-module $\mathcal{M}$.

(6) If $P$ is aspherical and $\pi \neq 1$ then the components of $\partial P$ are aspherical.

(7) If $\pi$ has one end then the peripheral system is $\pi_{1}$-injective.

(8) $P$ is aspherical and the peripheral system is $\pi_{1}$-injective if and only if $\pi$ has one end and the components of $\partial P$ are aspherical. 
Proof. The first assertion follows from the long exact sequence of the pair $(P, \partial P)$, with coefficients $\mathbb{F}_{2}$, and duality.

If a boundary component $Y$ is aspherical then so are its preimages in $P^{+}$, and so we may assume that $P$ is orientable, for (2) and (3). Suppose first that $\partial P=Y$, and let $\iota_{Y}: Y \rightarrow P$ be the inclusion. We then have an exact sequence

$$
0 \rightarrow H_{2}(P ; \mathbb{Z}) \rightarrow H^{1}(P ; \mathbb{Z}) \rightarrow H_{1}(Y ; \mathbb{Z}) \rightarrow H_{1}(P ; \mathbb{Z}) \rightarrow H^{2}(P ; \mathbb{Z}) \rightarrow 0 .
$$

If some component of $\partial \widetilde{P}=p^{-1}(Y)$ is closed then the image of $\pi_{1}(Y)$ in $\pi$ is finite. But then $H_{1}(Y ; \mathbb{Z})=0$, contrary to the assumption that $Y$ is aspherical.

It is clear that $B=\operatorname{Im}\left(H_{1}\left(\iota_{Y}\right)\right)$ and $\operatorname{Ker}\left(H_{1}\left(\iota_{Y}\right)\right)$ each have rank $\beta_{1}(P)-\beta_{2}(P)$. Moreover, as $H_{1}(Y ; \mathbb{Z})$ is torsion-free and the torsion subgroups of $H_{1}(P ; \mathbb{Z})$ and $H^{2}(P ; \mathbb{Z})$ have the same cardinality, by the Universal Coefficient Theorem, $B$ is a free direct summand of $H_{1}(P ; \mathbb{Z})$. Hence $\beta_{1}(Y)=2 \operatorname{rank}(B)$.

In general, we may reduce to the case when $\partial P=Y$, by capping off the other components of $\partial P$ with handlebodies. Although these reductions may replace $\pi$ by a quotient group, we may conclude that the preimages of $Y$ in some intermediate covering of $P$ are open surfaces, and (2) then follows. In (3) the equality of the previous paragraph may become an inequality.

Since $\partial \widetilde{P}$ is a union of open surfaces, (4) is immediate.

The remaining assertions follow from duality with coefficients in more general modules. Since $\partial P$ is nonempty, $H^{3}(P ; \mathcal{M})=H_{0}(P, \partial P ; \mathcal{M})=0$ for any coefficient module $\mathcal{M}$. Assertions (6)-(8) follow similarly from the long exact sequence with coefficients $\mathbb{Z}[\pi]$ and duality.

Let $K=P \cup e^{\geqslant 3}$ be a $K(\pi, 1)$-complex obtained by adjoining cells of dimension $\geqslant 3$ to kill higher homotopy groups of $P$. Then inclusion defines a map of pairs $c_{P}:(P, \partial P) \rightarrow(K, \partial P)$. Let

$$
\mu_{P}=c_{P *}[P, \partial P] \in H_{3}\left(\pi,\left\{\kappa_{i}\right\} ; \mathbb{Z}^{w}\right)=H_{3}\left(K, \partial P ; \mathbb{Z}^{w}\right) .
$$

The fundamental triple of the pair is $\left[\left\{\kappa_{i}\right\}, w_{1}(P), \mu_{P}\right]$. (When the components of $\partial P$ are aspherical and the peripheral system is $\pi_{1}$-injective, the group $H_{3}\left(\pi,\left\{\kappa_{i}\right\} ; \mathbb{Z}^{w}\right)$ may be defined in purely algebraic terms. See $\$ 8$ of Chapter 1.)

Two such triples $\left[\left\{\kappa_{i}\right\}, w, \mu\right]$ and $\left[\left\{\widehat{\kappa_{i}}\right\}, \widehat{w}, \widehat{\mu}\right]$ are equivalent if there are isomorphisms $\theta$ (of the ambient groups) and $\theta_{i}$ (of the domains of the peripheral system) such that $\theta \kappa_{i}$ is conjugate to $\widehat{\kappa_{i}} \theta_{i}$ for all $i$, $w=\widehat{w} \theta$ and $\theta_{*} \mu= \pm \widehat{\mu}$.

Bleile extended Turaev's arguments to give a Classification Theorem for pairs.

Theorem [12]. Two $\mathrm{PD}_{3}$-pairs $(P, \partial P)$ and $(Q, \partial Q)$ with aspherical boundary components are homotopy equivalent if and only if their fundamental triples are equivalent.

She also extended the Realization Theorem, with a restriction on the boundary conditions. We shall not prove the Classification Theorem for pairs, but we shall prove her version of the Realization Theorem in the next section.

When $P$ is an irreducible, orientable 3 -manifold with nonempty, $\pi_{1}$-injective boundary it is determined up to a finite ambiguity by $\pi$ alone; if, moreover, $P$ has no essential annuli then it is the only 3-manifold with fundamental group $\pi$ [AFW, Chapter 2.2]. No corresponding results for $\mathrm{PD}_{3}$-complexes are yet known. 


\subsection{A Realization Theorem for certain pairs}

The central invariant of the Realization Theorem is Turaev's homomorphism $v_{C *, 2}$ (defined in $\S 5$ of Chapter 2), which requires that $C_{*}$ be a free chain complex which is finitely generated in degrees $\leqslant 2$. The cellular chain complex of a $\mathrm{PD}_{3}$-space $P$ may not have this property, but is always chain homotopy equivalent to such a complex. The first lemma ensures that the criterion of the Realization Theorem does not depend on the choice of such a complex within the chain homotopy type.

Lemma 3.2 [147, Lemma 4.1.2]. Let $C_{*}$ and $D_{*}$ be free chain complexes which are finitely generated in degrees $\leqslant r$. If $f: C_{*} \rightarrow D_{*}$ is a homomorphism such that $H_{i}(f)$ is an isomorphism for all $i<r$, then $F^{r}(f)$ is a projective homotopy equivalence.

Proof. Suppose first that the homomorphisms $f_{i}: C_{i} \rightarrow D_{i}$ are isomorphisms, for $i<r$. Then $f_{r-1}\left(\partial C_{r}\right)=$ $\partial D_{r}$, since $H_{r-1}(f)$ is an isomorphism. Hence there is a homomorphism $g: D_{r} \rightarrow C_{r}$ such that $\partial f_{r} g=\partial$, since $D_{r}$ is a free module. Consider the diagram

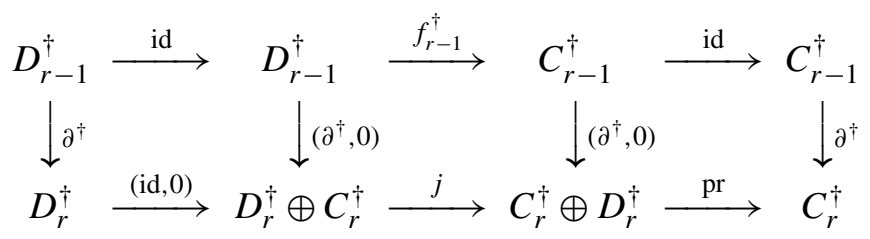

where

$$
j(x, y)=\left(f_{r}^{\dagger}(x)+y, x-g^{\dagger}\left(f_{r}^{\dagger}(x)+y\right)\right) \quad \forall x \in D_{r}^{\dagger} \text { and } y \in C_{r}^{\dagger},
$$

and where the vertical homomorphisms are the codifferentials. It is easy to see that $j$ is an isomorphism and the diagram commutes. Taking cokernels of the vertical homomorphisms, we get a sequence

$$
\operatorname{Coker}\left(\partial^{\dagger}\right) \rightarrow \operatorname{Coker}\left(\partial^{\dagger}\right) \oplus D_{r}^{\dagger} \cong D_{r}^{\dagger} \oplus \operatorname{Coker}\left(\partial^{\dagger}\right) \rightarrow \operatorname{Coker}\left(\partial^{\dagger}\right)
$$

with composite $F^{r}(f)$. Hence $F^{r}(f)$ is a projective homotopy equivalence.

In general, there is an inductive argument. We assume that for some $n \leqslant r$ the homomorphisms $f_{i}$ are isomorphisms for all $i<n$. Since $H_{n}\left(f_{*}\right)$ is an isomorphism $f_{*}$ also induces an isomorphism $C_{n} / \partial C_{n+1} \cong D_{n} / \partial D_{n+1}$, and since $D_{n}$ is free there are homomorphisms $g: D_{n} \rightarrow C_{n}$ and $\lambda: D_{n} \rightarrow D_{n+1}$ such that $\operatorname{id}_{D_{n}}-f_{n} g=\partial \lambda$. We define new complexes $\hat{C}_{*}$ and $\hat{D}_{*}$ by modifying $C_{*}$ and $D_{*}$ in degrees $n+1$ and $n$ only. We take the direct sum of $C_{*}$ with $\operatorname{id}_{D_{n}}: D_{n} \rightarrow D_{n}$, and similarly add $\operatorname{id}_{C_{n}}: C_{n} \rightarrow C_{n}$ to $D_{*}$. The new chain map $\hat{f}$ is defined by $\hat{f}_{i}=f_{i}$ if $i \neq n$ or $n+1$,

$$
\hat{f}_{n}(c, d)=\left(d+f_{n}(c-g(d)), c-g(d)\right) \quad \forall c \in C_{n} \text { and } d \in D_{n}
$$

and

$$
\hat{f}_{n+1}(c, d)=\left(f_{n+1}(c)+\lambda(d), \partial c-g(d)\right) \quad \forall c \in C_{n+1} \text { and } d \in D_{n+1} .
$$

The inclusion of $C_{*}$ into $\hat{C}_{*}$ and the projection of $\hat{D}_{*}$ onto $D_{*}$ induce homotopy equivalences of the modules $F^{i}$ for all $i$, and the lemma holds for $f_{*}$ if it holds for $\hat{f}_{*}$. Thus after a finite induction we are reduced to the case already treated. 
Suppose $C_{*}$ and $C_{*}^{\prime}$ are $\mathbb{Z}[\pi]$-chain complexes which are finitely generated in degrees $\leqslant 2$ and such that $H_{0}\left(C_{*}\right) \cong H_{0}\left(C_{*}^{\prime}\right) \cong \mathbb{Z}$ and $H_{1}\left(C_{*}\right)=H_{1}\left(C_{*}^{\prime}\right)=0$. If $f: C_{*} \rightarrow C_{*}^{\prime}$ is a chain homotopy equivalence such that $H_{0}(f)=\mathrm{id}_{\mathbb{Z}}$, we get as an immediate consequence of Lemma 3.2 that $v_{C_{*}, 2}=v_{C_{*}^{\prime}, 2} \circ F^{2}(f)$.

Lemma 3.3. Let $(P, \partial P)$ be a $\mathrm{PD}_{3}$-pair, with $\pi_{1}(P)=\pi$ and $w_{1}(P)=w$. Let é be a 0 -cell of $\widetilde{P}$, and let $x \in C_{3}(P, \partial P ; \mathbb{Z}[\pi])$ represent a generator of $H_{3}\left(P, \partial P ; \mathbb{Z}^{w}\right)$. Then there is a 1 -chain $v \in C_{1}(P ; \mathbb{Z}[\pi])$ such that

$$
\varphi \cap x=\overline{\varphi(x)}(\tilde{e}+\partial v),
$$

for all $\varphi \in C^{3}(P, \partial P ; \mathbb{Z}[\pi])$.

Proof. Let $\Delta$ be an equivariant diagonal approximation for $C_{*}(P ; \mathbb{Z}[\pi])$. Let $y \in C_{3}(P ; \mathbb{Z}[\pi])$ have image $x \in C_{3}(P, \partial P ; \mathbb{Z}[\pi])$, and suppose that $\Delta(y)=\sum y_{i} \otimes z_{3-i}$, with $y_{i}, z_{i} \in C_{i}(P ; \mathbb{Z}[\pi])$. Then $y=(\mathrm{id} \otimes \varepsilon) \Delta(y)$ implies that $y_{3} \varepsilon\left(z_{0}\right)=y$. As the image of $x$ generates $H_{3}\left(P, \partial P ; \mathbb{Z}^{w}\right) \cong \mathbb{Z}$, the chains $x$ and $y$ are indivisible, and so $\varepsilon\left(z_{0}\right)= \pm 1$. We may assume that $\varepsilon\left(z_{0}\right)=1$, so $y_{3}=y$, and $z_{0}-\tilde{e}=\partial v$ for some $v \in C_{1}(P ; \mathbb{Z}[\pi])$. Hence $\varphi \cap x=\overline{\varphi(x)}(\tilde{e}+\partial v)$, for all $\varphi \in C^{3}(P, \partial P ; \mathbb{Z}[\pi])$.

Let $G$ be a group and $\left\{\kappa_{i}: S_{i} \rightarrow G\right\}$ a finite family of homomorphisms. We may realize these by maps $f_{i}: Y_{i}=K\left(S_{i}, 1\right) \rightarrow K(G, 1)$. Let $K$ be the mapping cylinder of $\sqcup f_{i}: Y=\sqcup Y_{i} \rightarrow K(G, 1)$, and let $H_{i}\left(\pi,\left\{\kappa_{i}\right\} ; M\right)=H_{i}\left(M \otimes_{\mathbb{Z}[G]} C_{*}(K, Y ; \mathbb{Z}[G])\right)$ for any right $\mathbb{Z}[G]$-module $M$. If $G$ and the $S_{i}$ are all $F P_{2}$ the chain complex $C_{*}(K, Y ; \mathbb{Z}[G])$ is chain homotopy equivalent to a free complex which is finitely generated in degrees $\leqslant 2$. Let $w: G \rightarrow \mathbb{Z}^{\times}$be a homomorphism. We shall say that $\mu \in H_{3}\left(\pi,\left\{\kappa_{i}\right\} ; \mathbb{Z}^{w}\right)$ satisfies the Turaev condition if $v_{C_{*}, 2}(\mu)$ is a projective homotopy equivalence for some such chain complex $C_{*}$. (If so, it holds for any such complex, by Lemma 3.2.)

The next result shall become the criterion of the Realization Theorem. However, we do not assume here that the components of $\partial P$ are aspherical, or that the peripheral system is $\pi_{1}$-injective. (These hypotheses are needed later for our proof of the sufficiency of the criterion.)

Theorem 3.4. Let $(P, \partial P)$ be a $\mathrm{PD}_{3}$-pair, let $K=P \cup e^{\geqslant 3}$ be a $K(\pi, 1)$-complex obtained by adjoining cells of dimension $\geqslant 3$ to $P$, and let $\mu=c_{P *}[P, \partial P]$. Then $\mu$ satisfies the Turaev condition.

Proof. We shall assume first that $P$ has finite 2-skeleton. Then $C K P_{*}=C_{*}(K, \partial P ; \mathbb{Z}[\pi])$ is finitely generated in degrees $\leq 2$, since $K^{[2]}=P^{[2]}$ and $\partial P$ are finite. Let $C_{*}=C_{*}(P ; \mathbb{Z}[\pi])$ and $C_{*}^{\text {rel }}=$ $C_{*}(P, \partial P ; \mathbb{Z}[\pi])$. Cap product defines a chain homotopy equivalence from $C^{3-*}(P, \partial P ; \mathbb{Z}[\pi])$ to $C_{*}$, and hence (by Lemma 2.5 ) a projective homotopy equivalence

$$
F^{2}\left(C_{*}^{\text {rel }}\right)=G_{1}\left(C^{3-*}(P, \partial P ; \mathbb{Z}[\pi])\right) \simeq G_{1}\left(C_{*}\right) .
$$

Let $P^{[0]}=\left\{e_{i}^{0} \mid i \leqslant n\right\}$ be the 0 -skeleton of $P$ and let $\Delta_{0}$ be the submodule of $C_{0}$ with basis $\left\{e_{i}^{0}-e_{j}^{0} \mid j<i\right\}$. Then we may identify $C_{0} / \Delta_{0}$ with $\mathbb{Z}[\pi]$, since it has a canonical generator. Let $\left[\partial_{1}\right]$ be the composite of $\partial_{1}: C_{1} \rightarrow C_{0}$ with the projection onto $C_{0} / \Delta_{0}=\mathbb{Z}[\pi]$. The induced epimorphism $\vartheta: G_{1}\left(C_{*}\right) \rightarrow I(\pi)$ is a projective homotopy equivalence since it factors through $I(\pi) \oplus \mathbb{Z}[\pi]^{n-1}$.

Composition gives a projective homotopy equivalence

$$
F^{2}\left(C K P_{*}\right)=F^{2}\left(C_{*}^{\text {rel }}\right) \simeq G_{1}\left(C_{*}\right) \simeq I(\pi) .
$$


Lemma 3.3 together with the fact that $\cap x$ is a chain map gives an explicit formula for this homomorphism:

$$
[\varphi] \mapsto \vartheta \circ G_{1}(\varphi \cap x)=\overline{\partial^{2} \varphi(x)}\left(1+\left[\partial_{1}\right](v)\right),
$$

for all $[\varphi] \in F^{2}\left(C_{*}^{\text {rel }}\right)$. The Turaev homomorphism is given by

$$
v_{C K P_{*}, 2}(\mu)([\varphi])=\overline{\varphi\left(\delta_{2}(1 \otimes x)\right)},
$$

where $\delta_{2}: H_{3}\left(\mathbb{Z}^{w} \otimes_{\mathbb{Z}[\pi]} C_{*}\right) \rightarrow H_{2}\left(\bar{I} C_{*}\right)$ is the connecting homomorphism and $x$ is a 3-chain such that $1 \otimes x \in \mathbb{Z}^{w} \otimes_{\mathbb{Z}[\pi]} C_{3}$. It follows directly from the standard construction of $\delta_{*}$ that $\delta_{2}([1 \otimes x])=\left[\partial_{3} x\right]$. (Note that $\partial_{3} x$ is a 2-cycle for $\bar{I} C_{*}$, but is only a boundary in the larger complex $C_{*}$ !) Hence

$$
v_{C K P_{*}, 2}(\mu)([\varphi])=\overline{\varphi\left(\delta_{2}(1 \otimes x)\right)}=\overline{\delta^{2} \varphi(x)} .
$$

The homomorphism which sends $[\varphi]$ to $\overline{\partial^{2} \varphi(x)}\left[\partial_{1}\right](v) \in I(\pi)$ is null homotopic, since it factors through a map to $C_{1}$, and so $v_{C K P_{*}, 2}(\mu)$ is a projective homotopy equivalence.

In general, $C_{*}^{\text {rel }}$ is chain homotopy equivalent to a chain complex of free $\mathbb{Z}[\pi]$-modules which is finitely generated in degrees $\leqslant 2$, and therefore so is $C_{*}$, since $\partial P$ is a finite union of finite 2-complexes. We may then apply Lemma 3.2.

We would like to have an explicit characterization of the group systems that can be realized. The following partial extension of Theorem 2.4 is a step in this direction.

Corollary 3.4.1 [144]. Let $(P, \partial P)$ be a $\mathrm{PD}_{3}$-pair with nonempty boundary boundary, and let $\pi=\pi_{1}(P)$ and $\Omega=\pi_{0}(\partial \widetilde{P})$. Then

$$
[I(\pi)]=[D \Delta(\pi, \Omega)]
$$

Proof. Since projective homotopy equivalent modules are stably isomorphic (see $§ 1.1$ ), the theorem gives

$$
[I(\pi)]=\left[F^{2}\left(C_{*}^{\mathrm{rel}}\right)\right]=\left[D \operatorname{Coker}\left(\partial_{2}^{\mathrm{rel}}\right)\right] .
$$

Since $\partial P$ is nonempty, $H_{0}\left(C_{*}^{\text {rel }}\right)=0$, and so $\partial_{1}^{\text {rel }}$ splits. Therefore $\operatorname{Coker}\left(\partial_{2}^{\text {rel }}\right) \cong C_{0}^{\text {rel }} \oplus H_{1}(P, \partial P ; \mathbb{Z}[\pi])$. Now $H_{1}(P, \partial P ; \mathbb{Z}[\pi])$ is the kernel of the natural epimorphism

$$
H_{0}(\partial P ; \mathbb{Z}[\pi])=\mathbb{Z}[\Omega] \rightarrow H_{0}(P ; \mathbb{Z}[\pi])=\mathbb{Z},
$$

and so $H_{1}(P, \partial P ; \mathbb{Z}[\pi])=\Delta(\pi, \Omega)$. Hence $[I(\pi)]=\left[D \Delta(\pi, \Omega]\right.$. Since $H_{0}\left(Y_{j} ; \mathbb{Z}[\pi]\right) \cong \mathbb{Z}\left[B_{j} \backslash \pi\right]$, where $B_{j}=\operatorname{Im}\left(\kappa_{j}\right)$, for all $j \in J$, we have $H_{1}(P, \partial P ; \mathbb{Z}[\pi]) \cong \Delta\left(\pi,\left\{\kappa_{i}\right\}\right)$. Hence $[I(\pi)]=\left[D \Delta\left(\pi,\left\{\kappa_{i}\right\}\right)\right]$.

Let $K$ be a complex with a subcomplex $Y$ which is a closed surface, and let $w: \pi_{1}(K) \rightarrow \mathbb{Z}^{\times}$be a homomorphism whose restriction to each component $Y_{i}$ of $Y$ is $w_{1}\left(Y_{i}\right)$. A class $\mu \in H_{3}\left(K, Y ; \mathbb{Z}^{w}\right)$ satisfies the boundary compatibility condition if its image in $H_{2}\left(Y ; \mathbb{Z}^{w}\right)$ under the connecting homomorphism is a fundamental class for $Y$.

Theorem 3.5 (Realization Theorem [12; 147]). Let $\pi$ be an $\mathrm{FP}_{2}$ group, let $\left\{\kappa_{i}: S_{i} \rightarrow \pi \mid i \in I\right\}$ be a finite set of monomorphisms where the domains $S_{i}$ are $\mathrm{PD}_{2}$-groups, and let $w: \pi \rightarrow \mathbb{Z}^{\times}$be a homomorphism such that $w \circ \kappa_{i}=w_{1}\left(S_{i}\right)$, for all $i \in I$. Let $\mu \in H_{3}\left(\pi,\left\{\kappa_{i}\right\} ; \mathbb{Z}^{w}\right)$. Then the triple $\left[\left\{\kappa_{i}\right\}, w, \mu\right]$ is the fundamental triple of a $\mathrm{PD}_{3}$-pair with aspherical boundary components and $\pi_{1}$-injective peripheral system if and only if $\mu$ satisfies the boundary compatibility and Turaev conditions. 
Proof. Let $Y_{i}$ be a closed surface with fundamental group $S_{i}$, for all $i \in I$, and let $Z$ be a $K(\pi, 1)$ complex. The homomorphisms $\kappa_{i}$ may be realized by maps $f_{i}: Y_{i} \rightarrow Z$, and the mapping cylinder $K$ of $\sqcup f_{i}: Y=\sqcup Y_{i} \rightarrow Z$ is a $K(\pi, 1)$-complex which is as in the statement of the theorem. Let $p: \widetilde{K} \rightarrow K$ be the covering projection.

Suppose first that $\pi$ is finitely presentable. Then we may assume that $Z$ and $K$ have finite 2-skeleta, and set $C K Y_{*}=C_{*}\left(K, \sqcup Y_{i} ; \mathbb{Z}[\pi]\right)$. Since the $Y_{i}$ are aspherical and the $\kappa_{i}$ are monomorphisms, the inclusion

$$
C_{*}(K ; \mathbb{Z}[\pi]) \rightarrow C_{*}(K, Y ; \mathbb{Z}[\pi])
$$

induces isomorphisms on homology in degrees $\geqslant 2$. Hence

$$
\mathrm{H}_{2}\left(C K Y_{*}\right)=\mathrm{H}_{3}\left(C K Y_{*}\right)=0,
$$

and so $v_{C K Y_{*}, 2}$ is an isomorphism, by Lemma 2.6.

On the other hand, $H_{1}\left(C_{*}(K ; \mathbb{Z}[\pi])\right)=0$ and $H_{0}\left(C_{*}(K ; \mathbb{Z}[\pi])\right) \cong \mathbb{Z}$, whereas

$$
H_{1}\left(C K Y_{*}\right) \cong \Delta\left(\pi, \pi_{0}\left(p^{-1}(Y)\right)\right)
$$

and $H_{0}\left(C K Y_{*}\right)=0$. We note also that $K^{[1]}$ is a finite 1-complex, and $Y \subset K^{[2]}$.

It is clear from Lemma 3.2 and Theorem 3.4 that the conditions are necessary. Suppose that $\left[\left\{\kappa_{i}\right\}, w, \mu\right]$ is a triple for which $v_{C K Y_{*}, 2}(\mu)$ is a projective homotopy equivalence. Let $h: F^{2}\left(C K Y_{*}\right) \rightarrow I(\pi)$ be a homomorphism representing $v_{C K Y_{*}, 2}(\mu)$. Then we may factor $h$ as a composite

$$
h: F^{2}\left(C K Y_{*}\right) \rightarrow F^{2}\left(C K Y_{*}\right) \oplus \mathbb{Z}[\pi]^{m} \rightarrow I(\pi) \oplus L \rightarrow I(\pi),
$$

where $m<\infty$ and $L$ is a finitely generated projective module. After replacing $K$ by $K \vee m D^{3}$, where each 3-ball has the cellular decomposition $D^{3}=e^{0} \cup e^{2} \cup e^{3}$, we may assume $m=0$.

We now assume that $L$ is a free module of finite rank $n$. Let

$$
\phi: \mathbb{Z}[\pi]^{n+1}=\mathbb{Z}[\pi] \oplus L^{\dagger} \rightarrow C K Y_{2}
$$

be the homomorphism with dual $\phi^{\dagger}$ the composite

$$
C K Y_{2}^{\dagger} \rightarrow F^{2}\left(C K Y_{*}\right) \cong I(\pi) \oplus L \rightarrow \mathbb{Z}[\pi]^{n+1},
$$

and let $\operatorname{pr}_{1}$ and $\operatorname{pr}_{L}$ be the projections of $\mathbb{Z}[\pi] \oplus L$ onto its summands. Then $h([\psi])=\operatorname{pr}_{1} \phi^{\dagger}(\psi)=$ $\overline{\psi(\phi(x))}$, where $x=(1,0) \in \mathbb{Z}[\pi] \oplus L^{\dagger}$, for all $\psi \in C K Y_{2}^{\dagger}$.

Let $\partial=\partial_{2}: C K Y_{2} \rightarrow C K Y_{1}$. Since

$$
\partial \circ \phi=\left(\phi^{\dagger} \circ \partial^{\dagger}\right)^{\dagger}=0
$$

we see that $\operatorname{Im}(\phi) \leqslant \operatorname{Ker}(\partial)=H_{2}\left(K^{[2]}, Y ; \mathbb{Z}[\pi]\right)$. The inclusion of $K^{[2]}$ into $\left(K^{[2]}, Y\right)$ induces an isomorphism

$$
H_{2}\left(K^{[2]} ; \mathbb{Z}[\pi]\right) \cong \operatorname{Ker}(\partial)
$$

since the components of $p^{-1}(Y)$ are contractible. Let hwz $: \pi_{2}\left(K^{[2]}\right) \rightarrow \operatorname{Ker}(\partial)$ be the Hurewicz isomorphism for $\widetilde{K}^{[2]}$. Let $X=K^{[2]} \cup(n+1) e^{3}$, where the 3-cells are attached along the images of the 
standard basis for $\mathbb{Z}[\pi]^{n+1}$ under (hwz) ${ }^{-1} \circ \phi$. The inclusion of $K^{[2]}$ into $K$ extends to $c_{X}: X \rightarrow K$. Let $D_{*}=C_{*}(X, Y ; \mathbb{Z}[\pi])$. Then $D_{i}=C K Y_{i}$ for $i \leqslant 2$ and $D_{3}=\mathbb{Z}[\pi] \oplus L^{\dagger}$. Since $\operatorname{pr}_{L} \phi^{\dagger}$ is an epimorphism, $\varepsilon \otimes_{\mathbb{Z}[\pi]} \phi$ has rank $n$. Hence $H_{3}\left(X, Y ; \mathbb{Z}^{w}\right)=H_{3}\left(\mathbb{Z}^{w} \otimes D_{*}\right) \cong \mathbb{Z}$, with generator $[X, Y]$ represented by the 3 -cycle $1 \otimes x$. The image of $x \in D_{3}$ under the differential $\partial^{D}$ is $\phi(x)$, and so $\delta_{2}([1 \otimes x])=[\phi(x)] \in H_{2}\left(\bar{I} D_{2}\right)$.

Clearly $H^{i}(X ; \mathbb{Z}[\pi])=H_{3-i}\left(D_{*}\right)=0$ for $i=0$ or 2 , while $H^{3}(X ; \mathbb{Z}[\pi]) \cong \operatorname{Coker}(\phi)^{\dagger} \cong \mathbb{Z}$, and so

$$
\cap[X, Y]: H^{i}(X ; \mathbb{Z}[\pi]) \rightarrow H_{3-i}\left(D_{*}\right)
$$

is an isomorphism when $i \neq 1$. The case $i=1$ follows by the argument in the last paragraph of the proof of Theorem 2.1. Thus $(X, Y)$ is a $\mathrm{PD}_{3}$-pair with orientation character $w$ and fundamental class $[X, Y]$.

Since $1 \otimes x$ represents $[X, Y]$ and $\delta_{2}([1 \otimes x])=[\phi(x)]$ in $H_{2}\left(\bar{I} D_{2}\right), \operatorname{ev}_{2}\left(\delta_{2}(1 \otimes x)\right)=\operatorname{ev}_{2}(\phi(x))$ as elements of $\left[F^{2}\left(C K Y_{*}\right), I(\pi)\right]$. Hence $v_{D_{*}, 2}([X, Y])=h$, and so

$$
v_{C K Y_{*}, 2}\left(c_{X *}[X, Y]\right)=v_{D_{*}, 2}([X, Y])=v_{C K Y_{*}, 2}(\mu) .
$$

Since $v_{C K Y_{*}, 2}$ is an isomorphism, $c_{X *}[X, Y]=\mu$. Thus $X$ realizes the triple $\left[\left\{\kappa_{i}\right\}, w, \mu\right]$.

In general, when $L$ is projective but not free (or when $\pi$ is $\mathrm{FP}_{2}$ but not finitely presentable), we must expect $X$ (or both $K^{[2]}$ and $X$ ) to have infinitely many 2- and 3-cells. Nevertheless, the construction and verification that $(X, Y)$ is a $\mathrm{PD}_{3}$-pair goes through as before. If $\pi$ is finitely presentable (but $L$ is not free), then $D_{*}$ is chain homotopy equivalent to a free complex which is finitely generated in every degree, and $X$ is homotopy equivalent to a cell complex with finite skeleta. Hence $X$ is finitely dominated [149].

\subsection{Connected sums}

There are two distinct notions of splitting for $\mathrm{PD}_{3}$-complexes with nonempty boundary, corresponding to the internal connected sum and boundary connected sum of 3-manifolds. Bleile has extended Turaev's Splitting Theorem in each case, for $\mathrm{PD}_{3}$-complexes with aspherical boundary components and $\pi_{1}$-injective peripheral systems. In order to formulate her results properly we need two definitions.

Let $\left\{\kappa_{1, j}: j \in J\right\}$ and $\left\{\kappa_{2, \ell}: \ell \in L\right\}$ be two finite systems of monomorphisms from $\mathrm{PD}_{2}$-groups into ambient groups $G_{1}$ and $G_{2}$, respectively. The free product is the system

$$
\left\{\iota_{1} \circ \kappa_{1, j}: j \in J\right\} \cup\left\{\iota_{2} \circ \kappa_{2, \ell}: \ell \in L\right\},
$$

where $\iota_{i}: G_{i} \rightarrow G_{1} * G_{2}$ is the canonical inclusion, for $i=1,2$. We say also that the resulting system decomposes as a free product.

Theorem (Decomposition Theorem I [12]). Let $(P, \partial P)$ be a finitely dominated $\mathrm{PD}_{3}$-pair with aspherical boundary components and $\pi_{1}$-injective peripheral system. Then $(P, \partial P)$ is the internal connected sum of two such pairs if and only if its peripheral system decomposes as a free product of two $\pi_{1}$-injective systems.

If $(P, \partial P)$ is as above and $\pi_{1}(P)$ is a nontrivial free product then the images of the terms of the peripheral system are indecomposable and not infinite cyclic, and so are each conjugate into one or the other of these factors, by the Kurosh Subgroup Theorem. Hence this Decomposition Theorem applies.

When $\partial P$ is empty, this reduces to the Splitting Theorem of Chapter 2. 
The peripheral system corresponding to forming the connected sum along a pair of boundary components $\partial P_{i}$ and $\partial Q_{k}$, for some $i \in J$ and $k \in L$, is the system

$$
\left\{\iota_{1} \circ \kappa_{1, j}: j \in J, j \neq i\right\} \cup\left\{\iota_{2} \circ \kappa_{2, \ell}: \ell \in L, \ell \neq k\right\} \cup\left\{\kappa_{\#}\right\},
$$

where $\kappa_{\#}: \pi_{1}\left(\partial P_{i} \sharp \partial Q_{k}\right) \rightarrow G_{1} * G_{2}$ is the composition of the canonical epimorphism with $\kappa_{1, i} * \kappa_{2, k}$.

Theorem (Decomposition Theorem II [12]). Let $(P, \partial P)$ be a finitely dominated $\mathrm{PD}_{3}$-pair with nonempty, aspherical boundary. Then $(P, \partial P)$ is the boundary connected sum of two pairs with aspherical boundary components and $\pi_{1}$-injective peripheral systems if and only if its peripheral system decomposes as a free product of two $\pi_{1}$-injective systems along a pair of boundary components.

Note that in this case the peripheral system of $(P, \partial P)$ is not $\pi_{1}$-injective. However, that condition is only needed in these two theorems to construct the summands; recognizing $(P, \partial P)$ as the sum then uses only the Classification Theorem for pairs.

\subsection{The Algebraic Loop Theorem}

Most of this section is taken from [23].

Lemma 3.6 [152, Lemma 3.3]. Let $(P, \partial P)$ be a $\mathrm{PD}_{3}$-pair. Then every component of $\widetilde{\partial P}$ is planar.

Proof. Let $i: \widetilde{\partial P} \rightarrow \widetilde{P}$ be the inclusion. Suppose that $\widetilde{\partial P}$ has a non-planar component $\widetilde{F}$. Since $\widetilde{F}$ is orientable there are simple closed curves $\alpha, \beta$ in $\widetilde{F}$ which meet transversely in just one point. As $\widetilde{P}$ is 1 -connected, $\beta$ bounds a 2-cycle, which defines a relative homology class in $H_{2}(P, \partial P ; \mathbb{Z}[\pi])$. Let $D \beta$ be the Poincaré dual class in $H^{1}(P ; \mathbb{Z}[\pi])$. Then $D \beta \cap i_{*}[\alpha]=i^{*} D \beta([\alpha])=1$, and so $i_{*}[\alpha] \neq 0$. This contradicts the 1-connectivity of $\widetilde{P}$.

Lemma 3.7 (Weak Loop Theorem). Let $(X, \partial X)$ be a $\mathrm{PD}_{3}$-pair. If $F$ is a component of $\partial X$ then there is a finite family of disjoint orientation-preserving simple loops $u_{1}, \ldots, u_{k}$ in $F$ and integers $n_{1}, \ldots, n_{k}>0$ such that the kernel of the homomorphism from $\pi_{1}(F)$ to $\pi_{1}(X)$ induced by the inclusion is normally generated by the classes $\left[u_{1}\right]^{n_{1}}, \ldots,\left[u_{k}\right]^{n_{k}}$.

Proof. Since the components of $\partial \tilde{X}$ are planar, the lemma follows from [107, Theorem 3].

The triangle group $T(m, n, p)$ is the group with presentation

$$
\left\langle x, y, z \mid x^{m}=y^{n}=z^{p}=1, x y z=1\right\rangle,
$$

where $m, n, p \geqslant 1$ are positive integers. It is spherical, flat or hyperbolic according as $\frac{1}{m}+\frac{1}{n}+\frac{1}{p}-1$ is positive, 0 , or negative, respectively. (Such groups are virtually free, and this quantity is $\chi^{\operatorname{virt}}(T(m, n, p)$.)

Lemma 3.8. Let $F$ be an oriented closed surface and let

$$
\Phi=\pi_{1}(F) /\left\langle\left[u_{1}\right]^{n_{1}}, \ldots,\left[u_{k}\right]^{n_{k}}\right\rangle
$$

where $u_{1}, \ldots, u_{k}$ is a nonempty finite family of disjoint simple loops in $F$ and $n_{1}, \ldots, n_{k}$ are positive integers. Suppose that the image of each $u_{i}$ in $\Phi$ is nontrivial. Then: 
(1) $\Phi$ is freely indecomposable.

(2) If $\Phi$ is virtually free and $e(\Phi)<\infty$ then $\Phi \cong \mathbb{Z} \oplus \mathbb{Z} / n \mathbb{Z}$ for some $n \geqslant 2$ (and in this case $F$ is a torus and $k=1$ ).

(3) If $\Phi$ is virtually free and $e(\Phi)=\infty$ then $\Phi$ is the fundamental group of a trivalent graph of groups with vertex groups isomorphic to spherical triangle groups $T(m, n, p)$ and cyclic edge groups, generated by conjugates of the standard generators $x, y, z$ in each triangle group.

Proof. We may assume that the order of the image of $u_{i}$ in $\Phi$ is $n_{i} \geqslant 2$, for each $i \leqslant k$. We may also assume that no two loops $u_{i}, u_{j}$ are parallel. Cut $F$ open along the loops $u_{i}$ to obtain a collection of bounded surfaces $F_{j}$, with $1 \leqslant j \leqslant m$, and attach discs to each boundary component via degree- $d$ maps, where $d=n_{i}$ if the boundary component arose from cutting along $u_{i}$. We obtain a finite family of 2-orbifolds $\widehat{F}_{1}, \ldots, \widehat{F}_{m}$. Let $\widehat{F}=F \cup k e^{2}$ be the 2-complex obtained from $F$ by adjoining 2-discs $D_{i}$ via degree- $n_{i}$ maps from $\partial D_{i}$ onto $u_{i}$, for $i \leqslant k$. Let $\Gamma$ be the graph with vertices the components of $F \backslash\left\{u_{1}, \ldots, u_{k}\right\}$ and edges $\left\{u_{1}, \ldots, u_{k}\right\}$, and let $(\mathcal{G}, \Gamma)$ be the graph of groups with vertex groups the 2-orbifold groups just described and edge groups the cyclic groups $\mathbb{Z} / n_{i} \mathbb{Z}$. Then $\Phi \cong \pi \mathcal{G}$.

Since the vertex groups are freely indecomposable and the edge groups are nontrivial, $\Phi$ is freely indecomposable.

If $\pi$ is virtually free then the vertex groups are all finite (for otherwise they are virtually $\mathrm{PD}_{2}$-groups). Hence they are either spherical triangle groups $T(m, n, p)$, or are finite cyclic. Since no pair of loops $u_{i}$ are parallel, the latter case can occur only if $F$ is a torus and $k=1$. Otherwise, the vertices all have valence 3 (corresponding to a pair-of-pants decomposition of $F$ ) and the associated groups are as asserted.

Lemma 3.9. If the group $\Phi$ of the previous lemma is virtually free then there is an element $g \in \Phi$ of prime order with infinite centralizer.

Proof. It suffices to find a finite subgroup with infinite normalizer. This is clear if $\Phi \cong \mathbb{Z} \oplus \mathbb{Z} / n \mathbb{Z}$ for some $n \geqslant 2$, so we may assume that $e(\Phi)=\infty$ and $\Phi \cong \pi \mathcal{G}$, as in Lemma 3.8.

Let $T=T(m, n, p)$, where $m \geqslant n \geqslant p$, and let $A$ be a cyclic subgroup of order $m$. If $m \geqslant 4$ then inspection shows that $A$ has index 2 in its normalizer. Thus if there is an edge group of order $\geqslant 4$ common to two vertex groups $T$ and $T^{\prime}$ then $N_{\Phi}(A)$ contains the infinite group $N_{T}(A) *_{A} N_{T^{\prime}}(A)$.

If there are no such edge groups then $(m, n, p)=(3,3,2),(3,2,2)$ or $(2,2,2)$, with corresponding vertex groups $A_{4}, S_{3}$ or $D_{4}=(\mathbb{Z} / 2 \mathbb{Z})^{2}$. The elements of $A_{4}$ of order 3 are all conjugate, and generate their own normalizers, while $S_{3}$ has an unique subgroup of order 3 . The subgroups of order 2 in $A_{4}$ and $D_{4}$ are properly contained in their normalizers.

The remaining case is when each edge group is its own normalizer in at least one of the adjacent vertex groups. In this case $\Gamma$ contains a path (i.e., a linear subgraph) connecting vertices $v$ and $w$ with extreme vertex groups $G_{v} \cong G_{w} \cong S_{3}$ and intermediate vertex groups $A_{4}$, and all edge groups of order 3 . The subgroup of $\Phi$ corresponding to this subgraph has the form $G_{v} *_{A} B *_{A^{\prime}} G_{w}$, where $A$ and $A^{\prime}$ have order 3 and $B$ is generated by all the intermediate vertex groups. Clearly $A$ is normal in $G_{v}$ and $A^{\prime}$ is normal in $G_{w}$. The images of $A$ and $A^{\prime}$ are conjugate, and so $A$ is also normalized by a conjugate of $G_{w}$. It follows easily that $A$ has infinite normalizer.

We now state the main result of this section. (The argument uses one result from Chapter 4.) 
Theorem 3.10 (Algebraic Loop Theorem [23]). Let $(P, \partial P)$ be a $\mathrm{PD}_{3}$-pair. If $\partial P$ has a component $F$ such that the homomorphism from $\pi_{1}(F)$ to $\pi_{1}(P)$ induced by the inclusion is not injective then there is an orientation-preserving, essential simple loop $u$ in $F$ which is null-homotopic in $P$.

Proof. Let $\Gamma_{F}$ be the image of $\pi_{1}(F)$ in $\pi=\pi_{1}(P)$. Then there is a nonempty finite family of disjoint orientation-preserving simple loops $u_{1}, \ldots, u_{k}$ in $F$ and integers $n_{1}, \ldots, n_{k}>0$ such that

$$
\Gamma_{F} \cong \pi_{1}(F) /\left\langle\left[u_{1}\right]^{n_{1}}, \ldots,\left[u_{k}\right]^{n_{k}}\right\rangle,
$$

by Lemma 3.7. We may assume that each $u_{i}$ is essential in $F$. Suppose that they are all essential in $P$, and suppose also that $P$ is orientable. Double $P$ along $\partial P$ to obtain an orientable $\mathrm{PD}_{3}$-complex $Z=P \cup_{\partial P} P^{\prime}$, where $P^{\prime}$ is a second copy of $P$. Let $\Gamma$ be the graph with two vertices $v, w$ and $\left|\pi_{0}(\partial P)\right|$ edges connecting these vertices, and let $(\mathcal{G}, \Gamma)$ be the graph of groups with vertex groups $G_{v}=\pi$ and $G_{w}=\pi_{1}\left(P^{\prime}\right) \cong \pi$, and edge groups the images of the fundamental groups of the boundary components (with the associated inclusions). Then $\pi_{1}(Z) \cong \pi \mathcal{G}$.

The closed $\mathrm{PD}_{3}$-complex $Z$ is homotopy equivalent to a connected sum $V \sharp P_{1} \sharp \cdots \sharp P_{r}$, where each summand $P_{i}$ is aspherical and $\pi_{1}(V)$ is virtually free. Thus $\pi_{1}(Z) \cong \pi_{1}(V) * G_{1} * \cdots * G_{r}$, where $G_{i}=\pi_{1}\left(P_{i}\right)$, for $i \leqslant r$.

Since $\Gamma_{F}$ is indecomposable it is either infinite cyclic or is conjugate into one of the factors, by the Kurosh Subgroup Theorem. Since $\Gamma_{F}$ has nontrivial torsion it must be conjugate into $\pi_{1}(V)$, and so is virtually free. But it has elements of prime order which have infinite centralizer in $\pi_{1}(Z)$, by Lemma 3.9. This contradicts the Centralizer Condition of Chapter 4.

If $X$ is not orientable we choose a component $F^{\prime}$ of $\partial P^{+}$which covers $F$. Each loop $u_{i}$ lifts to a simple loop in $F^{\prime}$, and the above argument shows that one of these lifts is null-homotopic in $P^{+}$. Hence its image in $F$ (which is a simple loop) is null-homotopic in $P$.

Corollary 3.10.1. Let $(P, \partial P)$ be a $\mathrm{PD}_{3}$-pair. Then there is a finite family of disjoint (essential) simple loops $u_{1}, \ldots, u_{k}$ in $\partial P$ such that attaching $D^{2} \times[-1,1]$ along a neighbourhood of each loop yields a $\mathrm{PD}_{3}$-pair $(\widehat{P}, \partial \widehat{P})$ with the same fundamental group and such that each component of $\partial \widehat{P}$ is $\pi_{1}$-injective.

Proof. If $F$ is a non-aspherical boundary component then either $F \cong S^{2}$ or $F \cong R P^{2}$. In the latter case $P$ is nonorientable, and $w_{1}(P)$ splits the homomorphism $\mathbb{Z} / 2 \mathbb{Z} \rightarrow \pi$ induced by the inclusion of $F$ into $P$. Thus such components are $\pi_{1}$-injective.

For all other components a simple inductive argument using $|\chi(F)|$ shows that after finitely many applications of the theorem we may modify $F$ by elementary surgeries so that it is $\pi_{1}$-injective.

The next corollary follows from the Algebraic Loop Theorem and the argument for 3-manifolds in [76, Proposition 1.1].

Corollary 3.10.2. Let $(P, \partial P)$ be a $\mathrm{PD}_{3}$-pair, with aspherical boundary components and peripheral system $\left\{\kappa_{j}: j \in J\right\}$. Then $\operatorname{Im}\left(\kappa_{j}\right)$ is a free product of $\mathrm{PD}_{2}$-groups, copies of $\mathbb{Z} / 2 \mathbb{Z}$ and a free group.

In [71] we show that the $\pi_{1}$-injectivity condition in Theorem 3.5 may be replaced by the necessary conditions imposed by this corollary, if the images $\operatorname{Im}\left(\kappa_{j}\right)$ are all torsion free, and provided that the ambient group has a sufficiently large free factor. 


\subsection{Cubes with handles}

A cube with handles of genus $g$ is a 3-manifold $H$ obtained by attaching $g$ 1-handles to $D^{3}$. Thus $H \simeq \bigvee^{g} S^{1}$, and $H \cong \natural^{g} S^{1} \times D^{2}$ if it is orientable, while $H \cong \natural^{g} S^{1} \widetilde{\times} D^{2} \cong\left(S^{1} \widetilde{\times} D^{2}\right) \sharp^{g-1} S^{1} \times D^{2}$ otherwise. The boundary $\partial H$ is a closed surface with $\chi(\partial H)=2-2 g$, and is orientable if and only if $H$ is orientable. Every closed 3-manifold has a Heegaard decomposition as the union of two such cubes with handles (for all $g \gg 0$ ).

One might ask how far this extends to $\mathrm{PD}_{3}$-complexes. Are they unions $X \cup_{F} Y$ of $\mathrm{PD}_{3}$-pairs $(X, F)$ and $(Y, F)$ with $X \simeq Y \simeq \bigvee^{g} S^{1}$ ? We shall show that such $\mathrm{PD}_{3}$-pairs are homotopy equivalent rel $\partial$ to cubes with handles, and so $\mathrm{PD}_{3}$-complexes with such decompositions are homotopy equivalent to closed 3-manifolds.

Lemma 3.11. Let $(P, \partial P)$ be a $\mathrm{PD}_{3}$-pair with $\pi=\pi_{1}(P) \cong F(g)$. Then $\chi(P) \geqslant 1-g$, and $P$ is aspherical if and only if $\partial P \neq \varnothing$ and $\chi(P)=1-g$. In that case, $P \simeq \bigvee^{g} S^{1}, \partial P$ is connected, $\chi(\partial P)=2-2 g$ and the inclusion induces an epimorphism $\pi_{1}(\partial P) \rightarrow \pi_{1}(P)$.

Proof. If $\partial P$ is empty then $\chi(P)=0$, while if $\partial P \neq \varnothing$ then $H_{3}(P ; \mathbb{Z})=0$, and so $\chi(P) \geqslant 1-\beta_{1}(P)$. In each case, $\chi(P) \geqslant 1-g$, and $\chi(P)=1-g$ if $P$ is aspherical,

Let $\Gamma=\mathbb{Z}[F(g)]$, for simplicity of notation. If $\chi(P)=1-g$ and $\partial P \neq \varnothing$ then $C_{*}(P)$ is chain homotopy equivalent to a finite projective complex $C_{*}$ of length $\leqslant 2$, which is in fact free since projective $\Gamma$-modules are free [3]. On comparing the exact sequence

$$
0 \rightarrow \pi_{2}(P) \rightarrow C_{2} \rightarrow C_{1} \rightarrow C_{0} \rightarrow \mathbb{Z} \rightarrow 0
$$

with the standard $\Gamma$-resolution of $\mathbb{Z}$ we see that

$$
\pi_{2}(P) \oplus C_{1} \oplus \Gamma \cong C_{2} \oplus \Gamma^{g} \oplus C_{0},
$$

by Schanuel's Lemma. But $C_{1} \oplus \Gamma \cong C_{2} \oplus \Gamma^{g} \oplus C_{0}$, since $\chi(P)=1-g$. Therefore $\pi_{2}(P)=0$ and so $P$ is aspherical. Hence $P \simeq \bigvee^{g} S^{1}$.

Applying Poincaré duality with coefficients $\mathbb{F}_{2}$, we see that $\partial P$ is connected and $\chi(\partial P)=2-2 g$. Moreover, $H_{1}(P, \partial P ; \Gamma)=H^{2}(P ; \Gamma)=0$ and so $H_{0}(\partial \widetilde{P} ; \mathbb{Z}) \cong H_{0}(\widetilde{P} ; \mathbb{Z})$. Hence the homomorphism from $\pi_{1}(\partial P)$ to $\pi_{1}(P)$ induced by the inclusion is an epimorphism.

A similar result holds when $g=1$ and $\partial P \neq \varnothing$.

Jaco has shown that in the orientable case every such epimorphism is realized by the inclusion of the boundary of a cube with handles, and thus by a $\mathrm{PD}_{3}^{+}$-pair [75]. (In particular, $\operatorname{Aut}\left(\pi_{1}\left(T_{g}\right)\right.$ ) acts transitively on the set of epimorphisms from $\pi_{1}\left(T_{g}\right)$ to $F(g)$.)

Lemma [75, Lemma 4.1]. Let $F$ be a closed orientable surface of genus $g$ and let $f: \pi_{1}(F) \rightarrow F(g)$ be an epimorphism. Let $P$ be the mapping cylinder of a map $\phi: F \rightarrow \bigvee^{g} S^{1}$ such that $\pi_{1}(\phi)=f$. Then $(P, F) \simeq(H, \partial H)$, where $H$ is a cube with handles of genus $g$.

Theorem 3.12. Let $(P, \partial P)$ be a $\mathrm{PD}_{3}^{+}$-pair. If $P \simeq \bigvee^{g} S^{1}$ then $(P, \partial P) \simeq(H, \partial H)$, where $H$ is a cube with handles of genus $g$.

Proof. This follows immediately from Lemma 3.11 and [75, Lemma 4.1]. 
We may give a more self-contained argument for the theorem which extends to the nonorientable case (but leaves the issue of which epimorphisms are realizable aside) as follows. Let $F$ be a closed surface, and let $P$ be the mapping torus of a map $\phi: F \rightarrow \bigvee^{g} S^{1}$ which induces an epimorphism $\pi_{1}(\phi)$. Let $w=w_{1}(F)$. Straightforward applications of the long exact sequences of (co)homology with coefficients $\mathbb{Z}^{w}$ and then $\Gamma=\mathbb{Z}[F(g)]$ show that $H_{3}\left(P, F ; \mathbb{Z}^{w}\right) \cong H_{2}\left(F ; \mathbb{Z}^{w}\right)$, and that $H_{i}(P, F ; \Gamma)=0$ for $i \neq 2$, while $H^{j}(P ; \Gamma)=0$ for $j \neq 1$. Cup product with a generator $[P, F]$ of $H_{3}\left(P, F ; \mathbb{Z}^{w}\right)$ then induces duality isomorphisms if and only if restriction from $H^{1}(P ; \Gamma)$ to $H^{1}(F ; \Gamma)$ is an isomorphism (equivalently, if $\left.H^{2}(P, F ; \Gamma)=0\right)$. If $(P, \partial P)$ is a $\mathrm{PD}_{3}^{+}$-pair with $P \simeq \bigvee^{g} S^{1}$ then there is a simple closed curve $\gamma$ on $\partial P$ which is null-homotopic in $P$, by the Algebraic Loop Theorem or, more simply, by [133, Theorem 2]. Hence the homomorphism from $\pi_{1}(F)$ to $\pi_{1}(P)$ factors through $\pi_{1}\left(F_{1} \vee F_{2}\right)$, where $F_{1}$ and $F_{2}$ are closed surfaces with $\chi\left(F_{i}\right)=2-2 h_{i}>\chi(F)$ (if $\gamma$ separates $\partial P$ ) or through $\pi_{1}\left(F_{1} \vee S^{1}\right)$, where $\chi\left(F_{1}\right)>\chi(F)$ (if $\gamma$ does not separate $\partial P$ ). The images of the groups $\pi_{1}\left(F_{i}\right)$ in $F(g)$ are free, and their ranks are bounded by the maximal dimension of a self-orthogonal subspace of $H^{1}\left(F_{i} ; \mathbb{F}_{2}\right)$. We then see that they must be free factors of rank $h_{i}$, and the condition $H^{2}(P, F ; \Gamma)=0$ is inherited by the new pairs $\left(\bigvee^{h} S^{1}, F_{i}\right)$. We complete the argument by induction on $g$ and appeal to the Classification Theorem. This argument extends to $\mathrm{PD}_{3}$-pairs $(P, \partial P)$ with $\pi=\pi_{1}(P)$ free, all boundary components aspherical and $*_{i} \pi_{1}\left(\partial P_{i}\right)$ mapping onto $\pi$.

In the simplest case, when $\pi=1$, it is easy to see that $\partial P=\coprod^{\chi} S^{2}$ and $\pi_{2}(P) \cong \mathbb{Z}^{\chi-1}$, where $\chi=\chi(P)$. Hence $P \simeq \bigvee^{\chi-1} S^{2} \simeq \sharp^{\chi} D^{3}$. The group of self homotopy equivalences of $\sharp^{\chi} D^{3}$ is isomorphic to $\mathrm{GL}(\chi-1, \mathbb{Z})$, and so we may choose a homotopy equivalence $P \simeq \sharp^{\chi} D^{3}$ so that it induces a homotopy equivalence of pairs $(P, \partial P) \simeq\left(\sharp^{\chi} D^{3}, \coprod^{\chi} S^{2}\right)$. In [71] we show that every $\mathrm{PD}_{3}$-pair $(P, \partial P)$ with $\pi=\pi_{1}(P)$ a free group is homotopy equivalent to a 3 -manifold pair. 


\section{CHAPTER 4}

\section{The Centralizer Condition}

Let $P$ be a $\mathrm{PD}_{3}$-complex with infinite fundamental group $\pi$, and let $w=w_{1}(P)$. We may view Poincaré duality as giving an isomorphism of right modules $E(\pi)=H^{1}(P ; \mathbb{Z}[\pi]) \cong \overline{\pi_{2}(P)}$, and Theorem 1.2 gives us a short exact sequence presenting $E(\pi)$ as a quotient of $\mathbb{Z}[C]$-modules defined in terms of a graph of groups description of $\pi$. On the other hand, a very simple argument (Lemma 4.6) shows that if $C<\pi$ then $H_{s}\left(C ; \pi_{2}(P)\right) \cong H_{s+3}(C ; \mathbb{Z})$, for $s>1$. When we choose $C$ to be a subgroup of an edge group in a graph of groups description of $\pi$, comparison of these two aspects of the action of $C$ on $E(\pi)$ leads to strong restrictions on how $C$ sits in the neighbouring vertex groups, and ultimately on the edge groups.

This chapter is largely based on [22]. The first two sections consider the combinatorics of $G$-trees "with $\infty$-vertices". The results of these sections are used in $\S 3$, together with a simple lemma on the action of free automorphisms of a Moore space, to show that an indecomposable orientable $\mathrm{PD}_{3}$-complex is either aspherical or has virtually free fundamental group, and to derive Crisp's "Centralizer Condition". In Chapters 6 and 7 we shall see that the Normalizer and Centralizer conditions together lead to a very substantial understanding of $\mathrm{PD}_{3}$-complexes with virtually free fundamental groups.

\subsection{Trees with $\infty$-vertices and the invariant $\xi$}

Let $X$ be a tree, i.e., a contractible simplicial 1-complex. (We shall assume that $X$ is the realization of a graph with vertices $V X$ and edges $E X$, and well defined with origin and target functions $o$ and $t$.) Any two vertices $v, w$ determine a geodesic segment $[v, w]$ in $X$. A geodesic ray or line in $X$ is a subcomplex of $X$ which is homeomorphic to $[0, \infty)$ or the real line $\mathbb{R}$, respectively. Two geodesic rays $\rho, \rho^{\prime}$ are equivalent if $\rho \cap \rho^{\prime}$ is also a geodesic ray. The ends of $X$ are the equivalence classes of geodesic rays.

A $G$-tree is a tree $X$ with a simplicial left $G$ action such that $g \cdot o(e)=o(g \cdot e)$ and $g \cdot t(e)=t(g \cdot e)$ for all edges $e \in E X$. If $G \cong \pi \mathcal{G}$ where $(\mathcal{G}, \Gamma)$ is a graph of groups then the universal cover of $\widetilde{\Gamma}$ is a $G$-tree in which the stabilizers of the edges and vertices over $e$ and $v$ are conjugates of the images of $G_{e}$ and $G_{v}$, respectively. If $(\mathcal{G}, \Gamma)$ is reduced the corresponding $G$-tree is incompressible, in the terminology of [DD], and so this $G$-tree and $\mathcal{G}$ are essentially unique [DD, Proposition IV.7.4].

A $G$-tree $X$ is terminal if each edge stabilizer is finite and each vertex stabilizer is finite or has one end. If $G$ is finitely generated, then it is accessible if there is a terminal $G$-tree such that $\Gamma=G \backslash X$ is a finite graph.

A $G$-tree with $\infty$-vertices is a $G$-tree $X$ with a distinguished $G$-invariant subset $V_{f} X \subseteq V X$ of vertices of finite valence. Vertices not in $V_{f} X$ are said to be $\infty$-vertices. (We shall also use the expression "tree with $\infty$ vertices" when the group action is not relevant.) 
Assume now that $X$ is a tree with $\infty$-vertices, and let $|e(X)|$ and $|\infty(X)|$ be the numbers of ends and $\infty$-vertices of $X$, respectively. Let $\xi(X)=|e(X)|+|\infty(X)|-1$, and let $\Pi(X)$ be the cokernel of the $\operatorname{map} \Delta: \mathbb{Z}\left[V_{f} X\right] \rightarrow \mathbb{Z}[E X]$ given by

$$
\Delta(v)=\sum_{t(e)=v} e-\sum_{o(e)=v} e,
$$

where $\mathbb{Z}\left[V_{f}\right] X$ and $\mathbb{Z}[E X]$ are the free abelian groups with bases $V_{f} X$ and $E X$, respectively. If $X$ is a $G$-tree this is naturally a left $\mathbb{Z}[G]$-module. (The presentation in Theorem 1.2 looks very similar, but it involves right modules.) We shall call this the $G$-tree module presented by $X$.

Let $[e]_{X} \in \Pi(X)$ be the image of the edge $e$, for all $e \in E X$.

Theorem 4.1. Let $X$ be a tree with $\infty$-vertices. Then $\Pi(X)$ is a free abelian group of $\operatorname{rank} \max (\xi(X), 0)$.

Proof. Fix a vertex $*$ as a basepoint. We may assume that $X$ is oriented radially outwards from $*$, so that $o(e)$ is in the segment $[*, t(e)]$, for all edges $e$. For each vertex $v$ let $X_{v}$ be the subtree spanned by the vertices $w$ such that $v \in[*, w]$, and let

$$
E_{v}^{+}=\{e: o(e)=v\}
$$

be the set of edges starting at $v$. Let $X^{\prime}$ be the subgraph spanned by $*$ and the vertices $v$ for which either $X_{v}$ is infinite or contains an $\infty$-vertex. If $w$ is a vertex of $X^{\prime}$ other than $*$ then $[*, w] \subset X^{\prime}$, since $X_{w} \subset X_{u}$ for each $u \in[*, w]$. Hence $X^{\prime}$ is a subtree of $X$. The edges of $X^{\prime}$ are the edges $e$ such that either $X_{t(e)}$ is infinite or has an $\infty$-vertex. Thus if $e$ is not an edge of $X^{\prime}$ then $X_{t(e)}$ is a finite tree with no $\infty$-vertices. It is easily seen that $e=\sum_{v \in V X_{t(e)}} \Delta(v)$ in $\mathbb{Z}[E]$, and hence $[e]_{X}=0$. It follows that $\Pi(X) \cong \Pi\left(X^{\prime}\right)$.

Clearly $X=X_{*}$ is finite with no $\infty$-vertices if and only if $\xi(X)=-1$, and then $X^{\prime}=\{*\}$ is trivial. Hence $\Pi(X)=0$, so the theorem holds in this case. We may assume henceforth that $X_{*}$ is either infinite or contains an $\infty$-vertex.

If $v \in V X^{\prime}$ is not an $\infty$-vertex then $E_{v}^{+}$is finite, and $E X^{\prime} \cap E_{v}^{+}$is nonempty, for if $X_{t}(e)$ were finite with no $\infty$-vertices for all edges $e \in E_{v}^{+}$then the same would be true of $X_{v}$. Choose an edge succ $(v)$ in $E X^{\prime} \cap E_{v}^{+}$, for each $v \in V_{f} X^{\prime}$. We claim that

$$
\mathcal{E}=E X^{\prime} \backslash\left\{\operatorname{succ}(v): v \in V_{f} X^{\prime}\right\}
$$

represents a basis for the abelian group $\Pi\left(X^{\prime}\right)$. The claim follows immediately from the observation that each relation $\Delta(w)=0$ may be replaced by an equivalent relation which expresses $\operatorname{succ}(w)$ as a linear combination of edges in $\mathcal{E}$. This is clearly so if $w=*$. Otherwise, $w=t(e)$ for some $e \in E X^{\prime}$, and $\Delta(w)=0$ expresses $\operatorname{succ}(w)$ as a sum of signed elements of $\mathcal{E}$ and the edge $e$. The edge $e$ is either in $\mathcal{E}$ or may be assumed to be expressed as such a linear combination of elements of $\mathcal{E}$, by induction on the length of $[*, t(e)]$.

Let $\mathcal{P}$ be the union of the set of all geodesic rays starting from $*$ and the set of all geodesic segments $[*, z]$ with $z$ an $\infty$-vertex. Clearly $|\mathcal{P}|=\xi(X)+1$. When $z$ is an $\infty$-vertex every edge of $E_{z}^{+} \cap E X^{\prime}$ is in $\mathcal{E}$, and when $v \in V_{f} X^{\prime}$ every edge of $E_{v}^{+} \cap E X^{\prime}$ except $\operatorname{succ}(v)$ is in $\mathcal{E}$. Thus, for every $v \in V X^{\prime}$ there is a maximal subcomplex $p_{v}$ of $X^{\prime}$, which is a geodesic segment or ray starting at $*$, containing [*,v] and containing no edges of $\mathcal{E}$ not in $[*, v]$. In fact, $p_{v} \in \mathcal{P}$. 
Let $\mathcal{E}^{0}=\mathcal{E} \cup\{0\}$, and define a function $\pi: \mathcal{E}^{0} \rightarrow \mathcal{P}$ by $\pi(e)=p_{t(e)}$ and $\pi(0)=p_{*}$. Then $\pi(0)$ contains no edge in $\mathcal{E}$, while the edge of $\pi(e)$ which is furthest from $*$ among edges also in $\mathcal{E}$ is $e$. Hence $\pi$ is injective. When $\mathcal{E}$ is finite $\pi$ is also surjective. For if $\gamma \in \mathcal{P}$ then either no edge of $\gamma$ is in $\mathcal{E}$, in which case $\gamma=\pi(0)$, or $\gamma$ has only finitely many edges in $\mathcal{E}$, and then $\gamma=\pi(e)$ when $e$ is the edge furthest from $*$.

Thus if $|\mathcal{E}|$ is finite then so is $\xi(X)$, and $|\mathcal{E}|=\xi(X)$, while otherwise both $|\mathcal{E}|$ and $\xi(X)$ are infinite.

Corollary 4.1.1. If $G$ is an infinite group and $X$ is a terminal $G$-tree with $G \backslash X$ finite, then $|e(X)|+$ $|\infty(X)|$ is either 1, 2 or infinity, according to whether $G$ has 1, 2 or infinitely many ends.

Proof. Let $e(G)$ be the number of ends of $G$. Translating the presentation of the end module $E(G)$ given in Theorem 1.2 into the language of $G$-trees, and using the canonical anti-involution of $\mathbb{Z}[G]$ given by $g \mapsto g^{-1}$, we see that $\Pi(X) \cong \overline{E(G)}$. Since $G$ is infinite, $E(G)$ has rank $e(G)-1$ as an abelian group. The corollary now follows from the Theorem.

\subsection{The action of cyclic subgroups on a $G$-tree module}

Let $C=\langle g\rangle$ be a finite cyclic group of prime order $p$, and let $v_{p}(g)=\sum_{i<p} g^{i}$ be the norm element of $\mathbb{Z}[C]$. If $M$ is a $\mathbb{Z}[C]$-module let $M^{C}=\operatorname{Ker}\left((g-1) \mathrm{id}_{M}\right)$ and $M_{C}=M /(g-1) M$ be the associated invariant and coinvariant modules. Multiplication by $v_{p}(g)$ induces norm maps $N: M \rightarrow M^{C}$ and $\bar{N}: M_{C} \rightarrow M^{C}$, and $H_{i}(C ; M) \cong \operatorname{Ker}(N)$ or $\operatorname{Coker}(N)$, if $i>0$ and $i$ is even or odd, respectively. We shall compute these groups purely in terms of the subtree fixed by $C$, when $M=\Pi(X)$ for some $C$-tree $X$. (A key point is that the $\mathbb{Z}[C]$-modules arising are permutation modules.)

The fixed point set $X^{C}$ is a subtree of $X$, and setting $V_{f} X^{C}=\left(V_{f} X\right)^{C}$ makes $X^{C}$ into a tree with $\infty$-vertices. Let $A$ be the set of edges of $X$ which are not in $X^{C}$ but which have a vertex in $X^{C}$. Each connected component of $X \backslash X^{C}$ contains the interior of an unique element of $A$. For each $e \in A$ let $X_{e}$ be the tree (with $\infty$-vertices) which is the closure of the component of $X \backslash X^{C}$ containing the interior of $e$. Declare the new vertex to be an $\infty$-vertex. Let $B=\bigoplus_{e \in A} \Pi\left(X_{e}\right)$. Then $B$ is naturally a $\mathbb{Z}[C]$-module. Since $g \cdot e \neq e$ for each $e \in A$ and $g \cdot \Pi\left(X_{e}\right)$ is equal to $\Pi\left(X_{g(e)}\right)$, which is a free abelian group, $B$ is a free $\mathbb{Z}[C]$-module.

We shall think of $B$ as the module presented by the edges and vertices of $X$ outside $X^{C}$. Indeed $\Pi$ may be obtained from the $\mathbb{Z}[C]$-module $F=B \oplus \mathbb{Z}\left[E^{C}\right]$ by adjoining the remaining relations due to the vertices of $V_{f} X^{C}$. Let $\partial: \mathbb{Z}\left[V_{f} X^{C}\right] \rightarrow F$ be the homomorphism which maps $v \in V_{f} X^{C}$ to the image of $\sum_{e \mid t(e)=v} e-\sum_{e \mid o(e)=v} e$. Then $\Pi \cong \operatorname{Coker}(ð)$. Let $\phi: F \rightarrow \Pi$ be the corresponding quotient map, and write $D$ for $\operatorname{Ker}(\phi)=\partial\left(\mathbb{Z}\left[V_{f} X^{C}\right]\right)$. Each element of $D$ is fixed by $g$, since $g \cdot \partial(v)=\partial(g \cdot v)=\partial(v)$ for all $v \in V_{f} X^{C}$.

Since $B$ is a free $\mathbb{Z}[C]$-module, and $\mathbb{Z}\left[E^{C}\right]$ is a direct sum of copies of the augmentation module, the kernel of $v_{p}(g) \operatorname{id}_{F}$ is $(g-1) B$, while $v_{p}(g) F=B^{C} \oplus p \mathbb{Z}\left[E^{C}\right]$.

Lemma 4.2. Let $K$ be the submodule of $\Pi$ generated by the edges which do not lie in $X^{C}$. Then $K^{C}=$ $K \cap \Pi^{C} \leqslant \operatorname{Im}(N)$.

Proof. Suppose that $x \in B$ has image $\phi(x)$ in $K \cap \Pi^{C}$. Then $(g-1) x=\eta \in D$, and so must be fixed by $g$. Hence $p \cdot \eta=v_{p}(g) \eta=v_{p}(g)(g-1) x=0$, and so $\eta=0$, since $B$ is free as an abelian group. Thus $x \in B^{C}$ and hence $\phi(X) \in \operatorname{Im}(N)$. 
Lemma 4.3. The norm map $\bar{N}: \Pi_{C} \rightarrow \Pi^{C}$ has cokernel $(\mathbb{Z} / p \mathbb{Z})^{R}$, where $R=\max \left(\xi\left(X^{C}\right), 0\right)$ if $\xi\left(X^{C}\right)$ is finite, and $R$ is infinite otherwise.

Proof. Let $\psi: \Pi \rightarrow \Pi / K$ be the canonical projection. Since $\Pi / K \cong \Pi\left(X^{C}\right)$, it is free of rank $R$ as an abelian group. Since $\Pi=K+\Pi^{C}$, the restriction $\psi^{C}=\left.\psi\right|_{\Pi^{C}}$ is surjective, and $\operatorname{Ker}\left(\psi^{C}\right)=K \cap \Pi^{C}$. It now follows that $\operatorname{Coker}(\bar{N})=\operatorname{Coker}(N) \cong \operatorname{Coker}\left(\psi^{C} \circ N\right)$, since $K^{C} \leqslant \operatorname{Im}(N)$, by Lemma 4.2. Moreover $N(K) \leqslant K^{C}$, and so there is a well-defined homomorphism $N_{K}: \Pi / K \rightarrow \Pi / K$ such that $N_{K} \circ \psi=\psi^{C} \circ N$. Thus $\operatorname{Coker}\left(\psi^{C} \circ N\right)=\operatorname{Coker}\left(N_{K}\right)$. Since $\operatorname{Im}\left(N_{K}\right)=p \Pi / K$, the lemma folllows.

Lemma 4.4. The kernel of the norm map $\bar{N}: \Pi_{C} \rightarrow \Pi^{C}$ is $\mathbb{Z} / p \mathbb{Z}$ if $\xi\left(X^{C}\right)=-1$, and is 0 otherwise.

Proof. Consider this commuting square, in which $\psi$ denotes the canonical projection of $\Pi$ onto $\Pi_{C}$ :

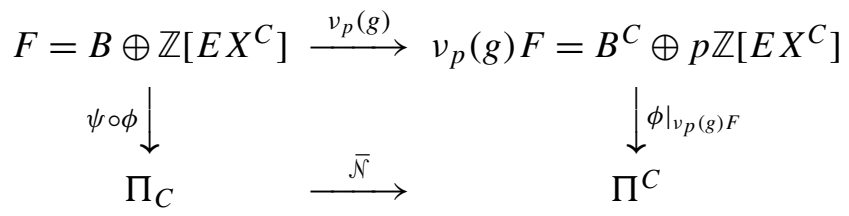

Note that $D+(g-1) B) \leqslant \operatorname{Ker}(\psi \circ \phi)$. Conversely, if $\phi(x) \in \operatorname{Ker}(\phi)=(g-1) \Pi$ then $\phi(x)=(g-1) \phi(y)=$ $\phi((g-1) y)$ for some $y \in F$, and so $x \in D+(g-1) B$. Hence $\operatorname{Ker}(\psi \circ \phi)=D+(g-1) B$, and so $\psi \circ \phi$ induces an epimorphism $\phi^{\prime}: B^{C} \oplus p \mathbb{Z}\left[E X^{C}\right] \rightarrow \Pi_{C}$, with kernel $v_{p}(g) D$, or simply $p D$, since elements of $D$ are fixed by $g$. It now follows from the diagram that

$$
\operatorname{Ker}(\bar{N})=\phi^{\prime}\left(\operatorname{Ker}\left(\left.\phi\right|_{B^{C} \oplus p \mathbb{Z}\left[E X^{C}\right]}\right)\right)=\phi^{\prime}\left(D \cap\left(B^{C} \oplus p \mathbb{Z}\left[E X^{C}\right]\right)\right),
$$

and this last group may be identified with the quotient $\left(D \cap\left(B^{C} \oplus p \mathbb{Z}\left[E X^{C}\right]\right)\right) / p D$.

Let $a \in D \cap\left(B^{C} \oplus p \mathbb{Z}\left[E^{C}\right]\right)$ have image $\alpha \in \operatorname{Ker}(\bar{N})$. We may assume that $a=\sum_{v \in V X^{C}} n_{v} \partial(v)$, where $0 \leqslant n_{v}<p$ for all $v \in V X^{C}$, and the sum is finite. The coefficient of each edge $e \in E X^{C}$ in this expression is $n_{t(e)}-n_{o(e)}$, and since $a$ is also in $B^{C} \oplus p \mathbb{Z}\left[E X^{C}\right]$ it follows that this coefficient must be a multiple of $p$. Hence $n_{t(e)}=n_{o(e)}$, since $0 \leqslant n_{v}<p$ for all $v$. Therefore the coefficients $n_{v}$ have a constant value $n$, for all $v \in V X^{C}$, since $X^{C}$ is connected. Now $n \neq 0$ only if $X^{C}$ is finite and has no $\infty$-vertices, that is, only if $\xi\left(X^{C}\right)=-1$, in which case putting $n=1$ gives a nontrivial element $\alpha$ of order $p$ which clearly generates $\operatorname{Ker}(\bar{N})$.

Theorem 4.5. Let $X$ be a $C$-tree with $\infty$-vertices. Let $R_{+}=\max \left(\xi\left(X^{C}\right), 0\right)$ if $\xi\left(X^{C}\right)$ is finite, and $R_{+}=\aleph_{0}$ otherwise, and let $R_{-}=\max \left(-\xi\left(X^{C}\right), 0\right)$. Then $H_{i}(C ; \Pi(X)) \cong(\mathbb{Z} / p \mathbb{Z})^{R_{+}}$for $i$ odd and $H_{i}(C ; \Pi(X)) \cong(\mathbb{Z} / p \mathbb{Z})^{R_{-}}$for $i$ even.

Proof. This follows immediately from Lemmas 4.3 and 4.4.

\subsection{Consequences for $\mathrm{PD}_{3}$-complexes}

We begin with a very simple lemma that has surprisingly strong consequences. In our main application, $Y$ is a $\mathrm{PD}_{3}$-complex, $\widehat{Y}=\widetilde{Y}$ and $p$ is prime. However, this lemma has obvious extensions to other situations.

Lemma 4.6. Let $Y$ be a cell complex and $\widehat{Y}$ a covering space such that $H_{q}(\widehat{Y})=0$ for $q \neq 0$ or 2 . If $g \in \operatorname{Aut}(\widehat{Y} / Y)$ has order $p<\infty$, then $H_{s}\left(\langle g\rangle ; H_{2}(\widehat{Y})\right)$ is isomorphic to $\mathbb{Z} / p \mathbb{Z}$ if $s>0$ is even and is 0 if $s$ is odd. 
Proof. Let $C_{*}=C_{*}(\widehat{Y} ; \mathbb{Z})$, considered as a chain complex over $\mathbb{Z}[C]$. Then dévissage applied to the homology of $C_{*}$ shows that $H_{s}\left(\langle g\rangle ; H_{2}(\widehat{Y})\right)=H_{s}\left(\langle g\rangle ; H_{2}\left(C_{*}\right)\right) \cong H_{s+3}(\langle g\rangle ; \mathbb{Z})$, for all $s>0$.

There is a subtle point in the next lemma. If $P$ is nonorientable, the $w$-twisted anti-involution involved in the Poincaré duality isomorphism $\pi_{2}(P) \cong \overline{H^{1}(P ; \mathbb{Z}[\pi])}=\overline{E(\pi)}$ is not the canonical one used in Corollary 4.1.1, which applies to all accessible groups.

Lemma 4.7. Let $P$ be a $\mathrm{PD}_{3}$-complex such that $\pi=\pi_{1}(P)$ is infinite, and let $w=w_{1}(P)$. Let $X$ be a terminal tree for $\pi$, and let $g \in \pi$ have prime order $p$.

(1) If $w(g)=1$ then $H_{s}(\langle g\rangle ; \Pi(X))$ is isomorphic to $\mathbb{Z} / p \mathbb{Z}$ if $s>0$ is even and is 0 if $s$ is odd.

(2) If $w(g)=-1($ so $p=2)$ then $H_{s}(\langle g\rangle$; $\Pi(X))$ is isomorphic to $\mathbb{Z} / 2 \mathbb{Z}$ if $s$ is odd and is 0 if $s>0$ is even.

Proof. If $w(g)=1$ then $\pi_{2}(P) \cong \overline{E(\pi)} \cong \Pi(X)$ as left $\mathbb{Z}[\langle g\rangle]$-modules, as outlined in Corollary 4.1.1, so the result follows from Lemma 4.6. If $w(g)=-1$ then $p=2$, and the $w$-twisted anti-involution differs only in sign from the canonical anti-involution used in Corollary 4.1.1. The result again follows from Lemma 4.6, with a shift of the degree as $g-1$ and $g+1$ are interchanged.

Theorem 4.5 and Lemma 4.7 together imply that if $X$ is a terminal $\pi$-tree and $g \in \pi$ has prime order $p$, then either $w(g)=1$ and $\xi\left(X^{\langle g\rangle}\right)=-1$ or $w(g)=-1$ and $\xi\left(X^{\langle g\rangle}\right)=1$.

Theorem 4.8. Let $P$ be an indecomposable orientable $\mathrm{PD}_{3}$-complex. Then either $P$ is aspherical or $\pi=\pi_{1}(P)$ is virtually free.

Proof. We may assume that $\pi$ is infinite. Then $P$ satisfies the hypotheses of Lemma 4.6. Let $X$ be a terminal $\pi$-tree and $(\mathcal{G}, \Gamma)$ the associated finite graph of groups. Since $P$ is indecomposable, $\pi$ is not a proper free product, and so all the edge groups are nontrivial.

Suppose that $\Gamma$ has at least one edge $e$. Choose an element $g \in G_{e}$ of prime order $p$. Since $P$ is orientable, $\xi\left(X^{\langle g\rangle}\right)=-1$, by the remark immediately before the theorem. Thus $X^{\langle g\rangle}$ is finite and has no $\infty$-vertices, and hence has finite vertex stabilizers. But $G_{o(e)}$ and $G_{t(e)}$ each stabilize a vertex of $X^{\langle g\rangle}$, and so must be finite groups. It follows immediately that either all vertex groups are finite, and so $\pi \cong \pi \mathcal{G}$ is virtually free, or $\Gamma$ is reduced to a single vertex $v$, with no edges, and $\pi=G_{v}$ has one end, in which case $P$ is aspherical.

Corollary 4.8.1. Let $P$ be a $\mathrm{PD}_{3}$-complex. Then $\pi_{1}(P)$ is virtually torsion-free.

The next result is central to our work in Chapters 6 and 7.

Theorem 4.9 (Centralizer Condition). Let $P$ be a $\mathrm{PD}_{3}$-complex. If $g \in \pi=\pi_{1}(P)$ has prime order $p$ and $C_{\pi}(\langle g\rangle)$ is infinite, then $p=2, w(g)=-1$ and $C_{\pi}(\langle g\rangle)$ has two ends.

Proof. Let $X$ be a terminal $\pi$-tree. If $x g=g x$ and $e$ is an edge of $X^{\langle g\rangle}$ then $g \cdot x \cdot e=x \cdot g \cdot e=x \cdot e$. Hence $C_{\pi}(\langle g\rangle)$ acts on $X^{\langle g\rangle}$, also with finite edge stabilizers. If $\xi\left(X^{\langle g\rangle}\right)=-1$ then $X^{\langle g\rangle}$ is a finite graph with finite vertex stabilizers in $\pi$. Since $\operatorname{Aut}\left(X^{\langle g\rangle}\right)$ is then finite, so is $C_{\pi}(\langle g\rangle)$, contrary to our hypothesis. Thus $\xi\left(X^{\langle g\rangle)}\right) \geqslant 0$. But then $p=2, w(g)=-1$ and $\xi\left(X^{\langle g\rangle}\right)=1$, by the remark immediately before Theorem 4.8. Thus either $X^{\langle g\rangle)}$ has two ends and no $\infty$-vertices, or it has one end and one $\infty$-vertex, or it is finite and has two $\infty$-vertices. There is an unique geodesic segment, ray or line $\gamma$ joining them in $X^{\langle g\rangle}$. Since $C_{\pi}(\langle g\rangle)$ respects the set of $\infty$-vertices and also acts on the set of ends, it must preserve $\gamma$. 
In fact, some subgroup $H$ of index at most 2 in $C_{\pi}(\langle g\rangle)$ must fix each end or $\infty$-vertex. If there is some $\infty$-vertex involved then the infinite group $H$ stabilizes every edge in $\gamma$, which is a contradiction. Thus $\gamma$ is a geodesic line joining two ends and $H$ acts by translations. For any edge $e$ in $\gamma$ the quotient $\left.H / \operatorname{stab}_{H}(e)\right)$ must be $\mathbb{Z}$. Since $\operatorname{stab}_{H}(e)$ is finite, $H$ and therefore $C_{\pi}(\langle g\rangle)$ each have two ends.

Since every element of the maximal finite normal subgroup of a group with two ends has infinite centralizer, the maximal finite normal subgroup of $C_{\pi}(\langle g\rangle)$ is $\langle g\rangle$, and since $w$ splits the inclusion of this subgroup, we must have $C_{\pi}(\langle g\rangle) \cong\langle g\rangle \times \mathbb{Z}$ or $\langle g\rangle \times D_{\infty}$.

Since the automorphism group of a finite group is finite this has the immediate consequence that if $G$ is a nontrivial finite subgroup of $\pi^{+}$then $N_{\pi}(G)$ is finite. In Corollary 7.10.2 we sharpen the final result of Theorem 4.9 to show that if $w(g)=-1$ and $C_{\pi}(g)$ is infinite then $C_{\pi}(g) \cong\langle g\rangle \times \mathbb{Z}$.

Corollary 4.9.1. Let $P$ be a $\mathrm{PD}_{3}$-space such that $\pi=\pi_{1}(P)$ has a nontrivial finite normal subgroup $N$. Then either $P \simeq R P^{2} \times S^{1}$ or $\pi$ is finite.

Proof. We may assume $\pi$ infinite. Then $C_{\pi}(N)$ has finite index in $\pi$, and so is infinite. Hence if $g \in N$ has prime order $p$ then $p=2, w(g)=-1$ and $N$ has two ends. Hence $N \simeq R P^{2} \times S^{1}[150]$.

This corollary was the original application of Lemma 4.6 [61].

If $\pi_{1}(P)$ has a finitely generated infinite normal subgroup of infinite index then it has one end, and so $P$ is aspherical. We shall discuss this case in Chapter 8.

If $Y$ is a $\mathrm{PD}_{3}^{+}$-complex with a regular covering space $\widehat{Y}$ such that $H_{1}(\widehat{Y} ; \mathbb{Z})=0$ and $G=\operatorname{Aut}(\widehat{Y} / Y)$ is infinite, then the hypotheses of Lemma 4.6 hold, and the argument for Corollary 4.9.1 in [61] extends to show that $G$ has no nontrivial finite normal subgroup. Moreover, $G$ is $\operatorname{FP}_{2}$, since the terms of $C_{*}(\widehat{Y} ; \mathbb{Z})$ in degrees $\leqslant 2$ give a partial $\mathbb{Z}[G]$-resolution of $\mathbb{Z}$ which is finitely generated and free. The arguments of this chapter imply that $G \cong\left(*_{i=1}^{r} G_{i}\right) * V$, where each factor $G_{i}$ has one end and $V$ is virtually free. Since $H_{2}(\widehat{Y}) \cong \overline{E(G)}$, by Poincaré duality, $\widehat{Y}$ is acyclic if and only if $G$ has one end. (In that case $G$ is a $\mathrm{PD}_{3}$-group.)

We now extend the partition of indecomposable orientable $\mathrm{PD}_{3}$-complexes to the bounded case.

Theorem 4.10. Let $(P, \partial P)$ be an orientable $\mathrm{PD}_{3}$-pair. Then $\pi=\pi_{1}(P)$ either has one end, is a proper free product, or is virtually free of finite rank. Moreover, if $g \in \pi$ has finite order then $C_{\pi}(\langle g\rangle)$ is finite.

Proof. The double of $P$ along its boundary is a $\mathrm{PD}_{3}$-complex $D P=P \cup_{\partial P} P$ [150, Theorem 2.1]. Since $P$ is a retract of $D P, \pi$ is a retract of $\pi_{1}(D P)$, and so is either free, a proper free product or a subgroup of one of the indecomposable free factors of $\pi_{1}(D P)$, by the Kurosh Subgroup Theorem. The claims now follow from Theorems 4.8 and 4.9.

Corollary 4.10.1. Let $(P, \partial P)$ be an indecomposable orientable $\mathrm{PD}_{3}$-pair such that the components of $\partial P$ are aspherical and $\pi_{1}$-injective. Then either $P$ is aspherical or $\pi=\pi_{1}(P)$ is virtually free and $\partial P=\varnothing$.

Proof. Since $(P, \partial P)$ is indecomposable, $\pi$ is not a proper free product, by the first Decomposition Theorem. (See §3.3.) If $\pi$ has one end then $H_{2}(\widetilde{P}, \partial \widetilde{P} ; \mathbb{Z})=\overline{E(\pi)}=0$. Since the components of $\partial \widetilde{P}$ are open surfaces, by Lemma $3.1, H_{2}(\partial \widetilde{P} ; \mathbb{Z})=0$ also. Hence $\pi_{2}(P)=H_{2}(\widetilde{P} ; \mathbb{Z})=0$, and so $P$ is aspherical. If $\pi$ is virtually free then $\partial P=\varnothing$, since $\mathrm{PD}_{2}$-groups are not subgroups of virtually free groups.

The corresponding result in the nonorientable case is not clear. 


\section{CHAPTER 5}

\section{Orientable $\mathrm{PD}_{3}$-complexes with $\pi$ virtually cyclic}

Indecomposable orientable $\mathrm{PD}_{3}$-complexes are either aspherical or have virtually free fundamental group. In the 3-manifold case the virtually free groups are either finite or have two ends, and are well understood. We shall consider $\mathrm{PD}_{3}$-complexes with such fundamental groups here, and the indecomposable orientable $\mathrm{PD}_{3}$-complexes with groups which are virtually nonabelian free in Chapter 6 . Nonorientable $\mathrm{PD}_{3}$-complexes are considered in Chapter 7.

Swan found examples of cell complexes $P$ with $\pi=\pi_{1}(P)$ finite and $\widetilde{P} \simeq S^{3}$ before the notion of Poincaré duality complex had been defined. These included $\mathrm{PD}_{3}$-complexes which are not homotopy equivalent to any closed 3-manifold. In $\S 1-\S 3$ we give the classifications of such complexes and their fundamental groups, and in $\S 4$ we give Swan's example with $\pi=S_{3}$, and show that its equivariant chain complex is self-dual. We describe the groups of (based) self homotopy equivalences of such complexes in $\S 5$, and consider finiteness and related issues briefly in $\S 6$. In the final section we show that if $\pi$ has two ends then $P$ is homotopy equivalent to one of four familiar 3-manifolds.

\subsection{Finite fundamental groups}

Let $P_{3}(X)$ denote the third stage of the Postnikov tower for the cell complex $X$. We may construct $P_{3}(X)$ by adjoining cells of dimension $\geqslant 5$ to kill off the higher homotopy groups. The second $k$-invariant $k_{2}(X)$ is the obstruction to finding a section to the projection of $P_{3}(X)$ to $P_{2}(X)$, and lies in $H^{4}\left(P_{2}(X) ; \pi_{3}(X)\right)$.

Lemma 5.1. Let $P$ be an orientable $\mathrm{PD}_{3}$-complex such that $\pi=\pi_{1}(P)$ is cyclic of prime order $p$. Then $k_{2}(P) \neq 0$.

Proof. Since $\widetilde{P}$ is also a $\mathrm{PD}_{3}$-complex, $\pi_{2}(P)=0$. Hence $P_{2}(P)=K(\pi, 1)$. The $k$-invariant $k_{2}(P)$ is the obstruction to finding a section to $c_{P_{3}(P)}: P_{3}(P) \rightarrow K(\pi, 1)$. Since $H_{3}\left(P_{3}(P) ; \mathbb{Z}\right) \cong \mathbb{Z}$ and $H_{4}\left(P_{3}(P) ; \mathbb{Z}\right)=0$, we have $H_{4}\left(P_{3}(P) ; \mathbb{F}_{p}\right)=0$ also, by the Universal Coefficient Theorem. But $H_{*}\left(\pi ; \mathbb{F}_{p}\right) \neq 0$ in every dimension, and so there is no map $s: K(\pi, 1) \rightarrow X$ such that $c_{X} s \sim \mathrm{id}_{K(\pi, 1)}$.

Our exposition of the classification of $\mathrm{PD}_{3}$-complexes with finite fundamental group is based on [150, Theorem 4.3].

Theorem 5.2. Let $P$ be a $\mathrm{PD}_{3}$-complex with $\pi=\pi_{1}(P)$ finite. Then:

(1) $\widetilde{P} \simeq S^{3}, P$ is orientable and $\pi$ has cohomological period dividing 4 .

(2) The homotopy type of $P$ is determined by $\pi$ and the orbit of the first nontrivial $k$-invariant $k_{2}(P)$ under $\operatorname{Out}(\pi) \times\{ \pm 1\}$.

(3) $k_{2}(P)$ generates $H^{4}(\pi ; \mathbb{Z}) \cong \mathbb{Z} /|\pi| \mathbb{Z}$. 
Proof. Since $\widetilde{P}$ is also a $\mathrm{PD}_{3}$-complex it is homotopy equivalent to $S^{3}$. Splicing together translates of $C_{*}(P ; \mathbb{Z}[\pi])$ gives a periodic resolution of the augmentation module $\mathbb{Z}$, with period 4 . Suppose that $P$ is nonorientable, and let $C$ be a cyclic subgroup of $\pi$ generated by an orientation-reversing element. Let $\widetilde{\mathbb{Z}}$ be the nontrivial infinite cyclic $\mathbb{Z}[C]$-module. Then $H^{2}\left(P_{C} ; \widetilde{\mathbb{Z}}\right) \cong H_{1}\left(P_{C} ; \mathbb{Z}\right) \cong C$, by Poincaré duality. But $H^{2}\left(P_{C} ; \widetilde{\mathbb{Z}}\right) \cong H^{2}(C ; \widetilde{\mathbb{Z}})=0$, since the classifying map from $P_{C}=\widetilde{P / C}$ to $K(C, 1)$ is 3-connected. Therefore $P$ must be orientable and $\pi$ must act trivially on $\pi_{3}(P) \cong H_{3}(\widetilde{P} ; \mathbb{Z})$.

We may construct the third stage of the Postnikov tower for $P$ by adjoining cells of dimension greater than 4 to $P$. The natural inclusion $j: P \rightarrow P_{3}(P)$ is then 4-connected. If $Q$ is another such $\mathrm{PD}_{3}$-complex and $\theta: \pi_{1}(Q) \rightarrow \pi$ is an isomorphism which identifies the $k$-invariants, then there is a 4-connected map $j_{1}: Q \rightarrow P_{3}(P)$ inducing $\theta$, which is homotopic to a map with image in the 4-skeleton of $P_{3}(P)$, and so there is a map $h: Q \rightarrow P$ such that $j_{1}$ is homotopic to $j h$. The map $h$ induces isomorphisms on $\pi_{i}$ for $i \leqslant 3$, since $j$ and $j_{1}$ are 4-connected, and so the lift $\tilde{h}: \widetilde{Q} \simeq S^{3} \rightarrow \widetilde{P} \simeq S^{3}$ is a homotopy equivalence, by the theorems of Hurewicz and Whitehead. Thus $h$ is itself a homotopy equivalence.

Inner automorphisms of $F$ act trivially on $H^{4}(\pi ; \mathbb{Z})$, while changing the orientation of $P$ corresponds to multiplication by -1 . Thus the orbit of $k_{2}(P)$ under $\operatorname{Out}(\pi) \times\{ \pm 1\}$ is the significant invariant. If $k_{2}(P)$ were not a generator for $H^{4}(\pi ; \mathbb{Z}) \cong \mathbb{Z} /|\pi| \mathbb{Z}$ then it would restrict to 0 for some cyclic subgroup $C$ of prime order. But this restriction is $k_{2}\left(P_{C}\right)$, which is nonzero by Lemma 5.1 .

Let $G$ be a finite group with cohomological period dividing 4 , and let $v_{G}=\sum_{g \in G} g$ be the norm element in $\mathbb{Z}[G]$. Let $K$ be a finite 2-complex with $\pi_{1}(K) \cong G$. A Schanuel's Lemma argument applied to $C_{*}(K ; \mathbb{Z}[G])$ and a periodic projective $\mathbb{Z}[G]$-resolution of $\mathbb{Z}$ shows that $\pi_{2}(K)=H_{2}(K ; \mathbb{Z}[G]) \cong$ $L \oplus \mathbb{Z}[G] /\left(v_{G}\right)$, where $L$ is a finitely generated projective left $\mathbb{Z}[G]$-module. If $L$ is free we may attach 3-cells to $K$ along a basis of $L$ and a generator for the final summand to obtain a finite 3-complex $P$ with universal cover $\simeq S^{3}$. If $L$ is not free let $W=\bigvee^{\mathbb{N}} S^{2}$ and attach countably many 3-cells to $K \vee W$. In each case the resulting complex is a $\mathrm{PD}_{3}$-complex, by Lemma 1.3. The construction may be varied to realize any generator of $H^{4}(G ; \mathbb{Z})$ as the first nontrivial $k$-invariant [136, Lemma 7.4]. In particular, there is an unique homotopy type of $\mathrm{PD}_{3}$-complexes with fundamental group $S_{3}=D_{6}$ (up to change of orientation).

\subsection{Finite groups with periodic cohomology}

A finite group has periodic cohomology if and only if its Sylow subgroups have periodic cohomology. This is in turn equivalent to the $p^{2}$-condition: all subgroups of order $p^{2}$ are cyclic, for any prime $p$. The $p$-subgroups are then cyclic, if $p$ is odd, while the 2-subgroups are cyclic or generalized quaternionic.

If $p, q$ are distinct primes, the group satisfies the $p q$-condition if every subgroup of order $p q$ is cyclic.

If all the Sylow subgroups of a finite group $M$ are cyclic then $M$ is metacyclic, with a presentation

$$
\left\langle a, b \mid a^{n}=b^{m}=1, a b a^{-1}=b^{r}\right\rangle,
$$

where $r^{n} \equiv 1 \bmod m$ and $(m, n(r-1))=1$, so $m$ is odd. (See [Ro, 10.1.10].) Let $u=\min \left\{k \mid r^{k} \equiv\right.$ $1 \bmod m\}$. Then $M^{\prime}$ and $\zeta M$ are generated by the images of $b$ and $a^{u}$, respectively. When $n=2$ and $r=-1$ we have the dihedral group $D_{2 m}$. If we set $m=2 s+1$ then $D_{2 m}$ has the presentation

$$
\left\langle a, b \mid a^{2}=1, a b^{s} a=b^{s+1}\right\rangle .
$$


The finite groups with cyclic or generalized quaternionic Sylow subgroups were determined by Suzuki and Zassenhaus. There are six families [AM, page 150]:

(1) $\mathbb{Z} / a \mathbb{Z} \rtimes \mathbb{Z} / b \mathbb{Z}$;

(2) $\mathbb{Z} / a \mathbb{Z} \rtimes\left(\mathbb{Z} / b \mathbb{Z} \times Q\left(2^{k}\right)\right), k \geqslant 3$;

(3) $\mathbb{Z} / a \mathbb{Z} \rtimes\left(\mathbb{Z} / b \mathbb{Z} \times T_{k}^{*}\right), k \geqslant 1$;

(4) $\mathbb{Z} / a \mathbb{Z} \rtimes\left(\mathbb{Z} / b \mathbb{Z} \times O_{k}^{*}\right), k \geqslant 1$;

(5) $(\mathbb{Z} / a \mathbb{Z} \rtimes \mathbb{Z} / b \mathbb{Z}) \times \operatorname{SL}(2, p), p \geqslant 5$ prime;

(6) $\mathbb{Z} / a \mathbb{Z} \rtimes(\mathbb{Z} / b \mathbb{Z} \times \mathrm{TL}(2, p)), p \geqslant 5$ prime.

Here $a, b$ and the order of the quotient by the metacyclic subgroup $\mathbb{Z} / a \mathbb{Z} \rtimes \mathbb{Z} / b \mathbb{Z}$ are relatively prime. The generalized quaternionic group $Q\left(2^{k}\right)$ and the extended binary polyhedral groups $T_{k}^{*}$ and $O_{k}^{*}$ have these presentations:

$$
\begin{aligned}
Q\left(2^{k}\right): & \left\langle x, y \mid x^{2^{k-2}}=y^{2}=(x y)^{2}\right\rangle, \\
T_{k}^{*}: & \left\langle Q(8), z \mid z^{3^{k}}=1, z x z^{-1}=y, z y z^{-1}=x y\right\rangle, \\
Q_{k}^{*}: & \left\langle T_{k}^{*}, w \mid w^{2}=x^{2}, w x w^{-1}=y x,, w z w^{-1}=z^{-1}\right\rangle .
\end{aligned}
$$

The groups $\mathrm{TL}(2, p)$ of the final family may be defined as follows. Choose a nonsquare $\omega \in \mathbb{F}_{p}^{\times}$, and let $\mathrm{TL}(2, p) \subset \mathrm{GL}(2, p)$ be the subset of matrices with determinant 1 or $\omega$. The multiplication $\star$ is given by $A \star B=A B$ if $A$ or $B$ has determinant 1 , and $A \star B=\omega^{-1} A B$ otherwise. Then $\operatorname{SL}(2, p)=\operatorname{TL}(2, p)^{\prime}$ and has index 2 . (Note also that $\operatorname{SL}(2,3) \cong T_{1}^{*}$ and $\operatorname{TL}(2,3) \cong O_{1}^{*}$.)

Lemma 5.3. Let $G$ be a finite group with periodic cohomology. If $G$ is not cyclic or metacyclic then 4 divides $|G|$, and $G$ has an unique element of order 2. This element is central, and is a square.

Proof. This follows on examining the above list of finite groups with periodic cohomology. Since all subgroups of order $p^{2}$ in a finite group $G$ with periodic cohomology are cyclic, an element $g \in G$ of order 2 is central if and only if it is the only element of order 2.

\subsection{Finite groups of cohomological period dividing 4}

A finite group has cohomological period 2 if and only if it is cyclic. If a finite group $G$ with periodic cohomology contains a semidirect product $\mathbb{Z} / m \mathbb{Z} \rtimes_{\theta} \mathbb{Z} / n \mathbb{Z}$, where $\theta$ has image of order $k$, then the cohomological period of $G$ is a multiple of $2 k$. (See [Br, Exercise VI.9.6].) In particular, metacyclic groups of odd order in family (1) which are not cyclic and all groups in families (5) and (6), excepting the direct products $\mathbb{Z} / a \mathbb{Z} \times \operatorname{SL}(2,5)$, have cohomological period greater than 4 .

The class of finite groups with cohomological period dividing 4 may be determined from the list given in $\$ 1$ above largely by excluding groups which contain metacyclic subgroups of odd order which are not cyclic. Gadgil gave a direct and simple argument for excluding such groups.

Lemma 5.4 [45]. Let $P$ be a $\mathrm{PD}_{3}$-complex such that $\pi=\pi_{1}(P)$ has order $p q$, where $p$ and $q$ are primes, and either $q=p$ or $p>q>2$. Then $\pi$ is cyclic. 
Proof. Suppose first that $q=p$. If $\pi$ is not cyclic then $\pi \cong(\mathbb{Z} / p \mathbb{Z})^{2}$. Since $\widetilde{P} \simeq S^{3}$, we have $\pi_{2}(P)=0$, and so $\beta_{2}\left(P ; \mathbb{F}_{p}\right)=\beta_{2}\left(\pi ; \mathbb{F}_{p}\right)=3$. But $\beta_{2}\left(P ; \mathbb{F}_{p}\right)=\beta_{1}\left(P ; \mathbb{F}_{p}\right)=2$, by Poincaré duality. Therefore $\pi \cong \mathbb{Z} / p^{2} \mathbb{Z}$.

Suppose now that $p>q$. Since the number of $p$-Sylow subgroups is 1 or $q$, and is congruent to $1 \bmod p$, the $p$-Sylow subgroup is normal. Hence we may assume that $\pi$ has a presentation

$$
\left\langle x, y \mid x^{p}=y^{q}=1, y x y^{-1}=x^{n}\right\rangle,
$$

for some $1 \leqslant n<p$ such that $n^{q} \equiv 1 \bmod p$. Let $P_{\langle x\rangle}$ be the regular covering space with fundamental group $\mathbb{Z} / p \mathbb{Z}$. The action of $\mathbb{Z} / q \mathbb{Z}$ on $P_{\langle x\rangle}$ preserves the orientation and the linking form, and induces multiplication by $n$ on $H_{1}\left(P_{\langle x\rangle}\right) \cong \mathbb{Z} / p \mathbb{Z}$. Therefore $n^{2} \equiv 1 \bmod p$, and so $n=1$, since $q$ is odd. Thus $\pi$ is abelian, and hence cyclic, since $(p, q)=1$.

Thus finite groups which are fundamental groups of $\mathrm{PD}_{3}$-groups satisfy the $p q$-condition for all odd primes $p, q$. This result is enough to determine the solvable groups of the Suzuki-Zassenhaus Theorem (classes (1)-(4) of the list in $\$ 2$ above) which can be fundamental groups of $\mathrm{PD}_{3}$-complexes.

Finite 3-manifold groups also satisfy the $2 p$-condition.

Theorem (2-Torsion Central Theorem [110]). If a group $G$ acts freely on a $\mathbb{Z} / 2 \mathbb{Z}$-homology 3-sphere then any element of order 2 is central. Hence $G$ has no dihedral subgroup $D_{2 p}$ with $p>1$.

Thus no 3-manifold has fundamental group $S_{3}$. This theorem needs some input from manifold topology, since there are $\mathrm{PD}_{3}$-complexes with $\pi \cong D_{2 p}$. See [103] for an alternative proof.

To handle the groups of types (5) and (6) in the list from [AM], we need to look at metacyclic subgroups of even order. Let $U(2, p)$ be the subgroup of upper triangular matrices in $\operatorname{SL}(2, p)$. Then $U(2, p)$ is a semirect product $\mathbb{Z} / p \mathbb{Z} \rtimes_{\rho} \mathbb{Z} /(p-1) \mathbb{Z}$, where $\rho$ acts through squares. The subgroup of $\operatorname{TL}(2,5)$ generated by $U(2,5)$ and $\left(\begin{array}{ll}1 & 0 \\ 0 & \omega\end{array}\right)$ is $\mathrm{TU}(2,5) \cong \mathbb{Z} / 5 \mathbb{Z} \rtimes_{2} \mathbb{Z} / 8 \mathbb{Z}$.

Lemma 5.5. Let $G$ be a finite group with periodic cohomology. Then $G$ has cohomological period greater than 4 if and only if it has a subgroup isomorphic to one of

(1) $\mathbb{Z} / p \mathbb{Z} \rtimes_{r} \mathbb{Z} / q \mathbb{Z}=\left\langle a, b \mid a^{q}=b^{p}=1, a b a^{-1}=b^{r}\right\rangle$, where $p$ is an odd prime, $q$ is an odd prime or 4 , $q$ divides $p-1$ and $r^{q} \equiv 1 \bmod p$ but $r^{2} \not \equiv 1 \bmod p ;$ or

(2) $\mathbb{Z} / 5 \mathbb{Z} \rtimes_{2} \mathbb{Z} / 8 \mathbb{Z}=\left\langle a, b \mid a^{8}=b^{5}=1, a b a^{-1}=b^{2}\right\rangle$.

Proof. This follows on applying the criterion of [Br, page 159] to the finite groups listed in $\S 2$ above.

A finite group has cohomological period 4 if and only if it is a product $B \times \mathbb{Z} / d \mathbb{Z}$ with $(|B|, d)=1$, where $B$ is

(1) $A(a, e)=\mathbb{Z} / a \mathbb{Z} \rtimes_{-1} \mathbb{Z} / 2^{e} \mathbb{Z}$ (of order $2^{e} a$ ), with presentation

$$
\left\langle x, y \mid x^{2^{e}}=y^{a}=1, x y x^{-1}=y^{-1}\right\rangle,
$$

where $a>1$ is odd and $e \geqslant 1$;

(2) $Q\left(2^{k} a, b, c\right)$ (of order $2^{k} a b c$ ), with presentation

$$
\left\langle Q\left(2^{k}\right), u \mid u^{a b c}=1, x u^{a b}=u^{a b} x, x u^{c} x^{-1}=u^{-c}, y u^{a c}=u^{a c} y, y u^{b} y^{-1}=u^{-b}\right\rangle,
$$

where $a, b$ and $c$ are odd and relatively prime, and either $n=3$ and at most one of $a, b$ and $c$ is 1 , 
or $n>3$ and $b c>1$;

(3) one of the groups $T_{k}^{*}$ (of order $\left.2^{3} 3^{k}\right), O_{k}^{*}\left(\right.$ of order $\left.2^{4} 3^{k}\right)$, or $I^{*}=\operatorname{SL}(2,5)$ (of order $2^{3} \cdot 3 \cdot 5$ ), or

(4) $P_{48 \cdot 3^{k-1} a}^{\prime \prime}=\mathbb{Z} / a \mathbb{Z} \rtimes_{-1} O_{k}^{*}$ (of order $2^{4} 3^{k} a$ ), with presentation

$$
\left\langle O_{k}^{*}, u \mid u^{a}=1, w u w^{-1}=u^{-1}, x, y, z \leftrightharpoons u\right\rangle,
$$

where $k \geqslant 1, a>1$ and $(a, 6)=1$.

The only finite groups with cohomological period 4 that have dihedral subgroups $D_{2 p}$ are the groups $A(m, 1)=D_{2 m}$ (with $\left.m>1\right)$, and their products with cyclic groups. These are also the only such groups with no central involution.

\subsection{A finite complex with fundamental group $S_{3}$}

Let $S_{3}=D_{6}$ be the symmetric group on 3 letters. There is a simple periodic resolution of the augmentation $\mathbb{Z}\left[S_{3}\right]$-module $\mathbb{Z}$ in the appendix to [136]. However we shall give our own account, which shall guide our treatment of $S_{3} * \mathbb{Z} / 2 \mathbb{Z} S_{3}$ in Chapter 6 .

The group $S_{3}$ has a presentation $\left\langle a, b \mid a^{2}, a b a b^{-2}\right\rangle$. Let $K$ be the corresponding 2-complex. Then $C_{*}(\widetilde{K})$ has the form

$$
\mathbb{Z}\left[S_{3}\right]^{2} \stackrel{\partial_{2}}{\longrightarrow} \mathbb{Z}\left[S_{3}\right]^{2} \stackrel{\partial_{1}}{\longrightarrow} \mathbb{Z}\left[S_{3}\right],
$$

where $\partial_{1}(1,0)=a-1, \partial_{1}(0,1)=b-1, \partial_{2}(1,0)=(a+1,0)$ and $\partial_{2}(0,1)=\left(b^{2} a+1, a-b-1\right)$. The 2-chain $\psi=\left(a-1,-b a+a+b^{2}-b\right)$ is a 2-cycle, and determines an element of $\pi_{2}(K)=H_{2}(\widetilde{K}$; $\mathbb{Z})$, by the Hurewicz Theorem. Let $X=K \cup_{\psi} e^{3}$. This construction is in [136], where it is shown that $\widetilde{X} \simeq S^{3}$ and that $C_{*}=C_{*}(\tilde{X})$ extends to a periodic resolution of $\mathbb{Z}$, with period 4 .

We shall define new bases which display the structure of $C_{*}$ to better advantage, as follows. Let $e_{1}=(1,0)$ and $e_{2}=\left(-b a-b^{2}, 1\right)$ in $C_{1}$ and $f_{1}=(1,0)$ and $f_{2}=(0,-a)$ in $C_{2}$, and let $g$ be the generator of $C_{3}$ corresponding to the top cell. Then $\partial_{1} e_{1}=a-1, \partial_{1} e_{2}=-b^{2} a+b a+b^{2}-1, \partial_{2} f_{1}=(a+1) e_{1}$, $\partial_{2} f_{2}=\left(b^{2} a+a-1\right) e_{2}$, and $\partial_{3} g=\psi=(a-1) f_{1}+\left(-b^{2} a+b a+b-1\right) f_{2}$. The matrix for $\partial_{2}$ with respect to the bases $\left\{\tilde{e}_{i}\right\}$ and $\left\{\tilde{f}_{j}\right\}$ is diagonal, and is hermitean with respect to the canonical involution of $\mathbb{Z}\left[S_{3}\right]$, while the matrix for $\partial_{3}$ is the conjugate transpose for that of $\partial_{1}$. Hence the chain complex $D C_{*}$ obtained by conjugating and reindexing the cochain complex $C^{*}\left(X ; \mathbb{Z}\left[S_{3}\right]\right)$ is isomorphic to $C_{*}$.

Let $B=\langle b\rangle \cong \mathbb{Z} / 3 \mathbb{Z}$, and let $\beta=b^{2}+b+1$ and $\gamma=\sum_{s \in S_{3}} s=\beta(a+1)$ be the norm elements in $\mathbb{Z}[B]$ and $\mathbb{Z}\left[S_{3}\right]$, respectively.

Lemma 5.6. The complex $X$ is a $\mathrm{PD}_{3}$-complex with $\widetilde{X} \simeq S^{3}$.

Proof. Since $C_{*}$ is the cellular chain complex of a 1-connected cell complex, we have $H_{0}\left(C_{*}\right) \cong \mathbb{Z}$ and $H_{1}\left(C_{*}\right)=0$. If $\partial_{2}\left(r f_{1}+s f_{2}\right)=0$ then $r(a+1)=0$ and $s\left(b^{2} a+a-1\right)=0$. Now the left annihilator ideals of $a+1$ and $b^{2} a+a-1$ in $\mathbb{Z}\left[S_{3}\right]$ are principal left ideals, generated by $a-1$ and $(b-1)(b a-1)$, respectively. Hence $r=p(a-1)$ and $s=q(b-1)(b a-1)$ for some $p, q \in \mathbb{Z}[B]$. A simple calculation gives $\partial_{3}((p(b a+b+1)+q(b a+b)) g)=r f_{1}+s f_{2}$ and so $H_{2}\left(C_{*}\right)=0$.

If $\partial_{3} h g=0$ then $h(a-1)=0$, so $h=h_{1}(a+1)$ for some $h_{1} \in \mathbb{Z}[B]$, and $h\left(b^{2} a-b a-b+1\right)=0$. Now $h\left(b^{2} a-b a-b+1\right)=h_{1}(1-b)(a+b+1)$, so $h_{1}(1-b)=0$. Therefore $h_{1}=m \beta$ for some $m \in \mathbb{Z}$, so $h=m \gamma$ and $H_{3}\left(C_{*}\right)=\mathbb{Z}\left[S_{3}\right] \gamma g \cong \mathbb{Z}$. Hence $\widetilde{X} \simeq S^{3}$. 
Now $H_{3}(X ; \mathbb{Z})=H_{3}\left(\mathbb{Z} \otimes_{\mathbb{Z}\left[S_{3}\right]} C_{*}\right)=\mathbb{Z}[1 \otimes g]$ and $\operatorname{tr}([1 \otimes g])=\gamma g$, where $\operatorname{tr}: H_{3}(X ; \mathbb{Z}) \rightarrow H_{3}(\tilde{X} ; \mathbb{Z})$ is the transfer homomorphism. The homomorphisms from $H^{q}\left(\overline{C^{*}\left(X ; \mathbb{Z}\left[S_{3}\right]\right)}\right)$ to $H_{3-q}\left(C_{*}\right)$ determined by cap product with $[X]=[1 \otimes g]$ may be identified with the Poincaré duality isomorphisms for $\tilde{X}$, and so $X$ is a $\mathrm{PD}_{3}$-complex.

The fact that $X$ is a $\mathrm{PD}_{3}$-complex is due to [150]. The only novelty here is the diagonalization of $\partial_{2}$, which is a guiding feature in the study of an example with $\pi=S_{3} *_{\mathbb{Z} / 2 \mathbb{Z}} S_{3}$, in Chapter 6 .

\subsection{Self homotopy equivalences}

In this section we shall assume that our $\mathrm{PD}_{3}$-complexes are based. Let $E^{*}(P)$ be the group of based homotopy classes of based self homotopy equivalences of $P$.

Theorem 5.7. Let $P$ be a $\mathrm{PD}_{3}$-complex such that $\pi=\pi_{1}(P)$ is finite, of order $|\pi|>2$. If $|\pi|$ is not divisible by 4 or by a prime $p \equiv 3 \bmod 4$, then

$$
E^{*}(P) \cong\left\{\alpha \in \operatorname{Aut}(\pi) \mid H^{4}(\alpha)= \pm 1\right\}
$$

otherwise,

$$
E^{*}(P) \cong\left\{f \in \operatorname{Aut}(\pi) \mid H^{4}(\alpha)=1\right\}
$$

Proof. If $h$ is a self homotopy equivalence of $P$ then $\pi_{1}(h)$ and $H_{3}(h)$ are automorphisms of $\pi$ and of $H_{3}(P) \cong \mathbb{Z}$, respectively. The corresponding automorphism of $H_{3}(\pi) \cong H^{4}(\pi ; \mathbb{Z}) \cong \mathbb{Z} /|\pi| \mathbb{Z}$ is multiplication by a square. To see this it is enough to consider automorphisms of Sylow subgroups. If the $p$-Sylow subgroup is cyclic, the result is clear. Automorphisms of $Q(8)$ induce the identity on $H^{4}(Q(8) ; \mathbb{Z})$ [136, Proposition 8.3], while automorphisms of quaternionic 2-groups $Q\left(2^{k}\right)$ of higher order induce multiplication by squares on $H^{4}\left(Q\left(2^{k}\right) ; \mathbb{Z}\right)$ [136, Proposition 8.2]. Hence $H_{3}(h)=1$ if $|\pi|$ is divisible by 4 or by a prime $p \equiv 3 \bmod 4$. Otherwise, $\pi$ is metacyclic, and $H_{3}(h)= \pm 1$.

If $f: P \rightarrow P$ is a based self-map such that $\pi_{1}(f)=\mathrm{id}_{\pi}$ then there are no obstructions to homotoping $f$ to a map which is the identity on $P_{o}$. We may homotope $f$ to the identity if and only if $\operatorname{deg} f=1$. Hence $\alpha$ is a monomorphism.

It remains for us to show that every such automorphism of $\pi$ is realizable by a map of the appropriate degree. Let $K=P \cup e^{\geqslant 4}$ be an Eilenberg-Mac Lane space constructed by adjoining higher-dimensional cells to $P$. We may realize any automorphism $\alpha$ of $\pi$ by a map from $P$ to $K$, and by cellular approximation, we may assume it has image in $P$. Suppose that $h: P \rightarrow P$ induces $\alpha=\pi_{1}(h)$, and that $H^{4}(\alpha)$ is multiplication by $\varepsilon= \pm 1$. Let $\tilde{h}$ be a lift of $h$ to a self-map of $\widetilde{P}$. Then $\tilde{h}$ has degree $\equiv \varepsilon \bmod |\pi|$. If $\operatorname{deg} \tilde{h}=\varepsilon+n|\pi|$, we may modify $h$ by taking its connected sum with a self-map of $S^{3}$ of degree $-n$. Let $h_{1}$ be the resulting self-map of $P \simeq P \sharp S^{3}$. Then $\pi_{1}\left(h_{1}\right)=\alpha$ and $\operatorname{deg} \tilde{h_{1}}=\operatorname{deg} \tilde{h}+|\pi|(-n)=\varepsilon$, and so $h_{1}$ is a self homotopy equivalence.

The two cases with smallest fundamental group need separate consideration, since orientation-reversing maps are not detected by $H_{3}(\pi)$ if $|\pi| \leqslant 2$. We have $P \simeq S^{3}$ when $\pi=1$ and $P \simeq R P^{3}$ when $\pi=\mathbb{Z} / 2 \mathbb{Z}$, and a degree-1 self-map is homotopic to the identity, as in the theorem. Since these spaces each have orientation-reversing involutions which fix a basepoint, $E^{*}(P)=\mathbb{Z}^{\times}$in each case. 


\subsection{Finiteness, standardness, and $\mathbb{S}^{3}$-manifolds}

Let $L_{\alpha}$ be the kernel of the projection of $\mathbb{Z}[G]$ onto $\mathbb{Z} / \alpha \mathbb{Z}$ (via the augmentation), where $\alpha \in \mathbb{Z}$. Swan showed that if $(\alpha,|G|)=1$ then $L_{\alpha}$ is a projective module, and the classes of such modules form a subgroup $T_{G} \leqslant \widetilde{K}_{0}(\mathbb{Z}[G])$. The Swan subgroup $T_{G}$ is a quotient of $(\mathbb{Z} /|G| \mathbb{Z}) / \pm 1$. The $k$-invariants realized by $\mathrm{PD}_{3}$-complexes lie in a coset of $T_{G}$, and so there is a finite $\mathrm{PD}_{3}$-complex with group $G$ if and only if an obstruction $\sigma_{4}(G) \in \widetilde{K}_{0}(\mathbb{Z}[G]) / T_{G}$ vanishes. The Swan obstruction $\sigma_{4}(G)$ is 0 if $G \cong B \times \mathbb{Z} / d \mathbb{Z}$, where $B=T_{k}^{*}, O_{1}^{*}, I^{*}$ or $A(m, e)$ (and $(|B|, d)=1$ ), but is nonzero if, for instance, $G=O_{k}^{*}$ with $k>1$ or $P_{48 r}^{\prime \prime}$ with $3 \mid r$. (See $[25 ; 151]$.)

If a $\mathrm{PD}_{3}$-complex $P$ is standard, it is homotopy equivalent to a finite 3-complex with one 0 -cell and one 3-cell. Since $\chi(P)=0$, the numbers of 1-cells and 2-cells agree, and so $\pi_{1}(P)$ has a balanced presentation. If $G$ is a finite group with cohomological period 4 and there are at most two quaternion factors $\mathbb{Z}$ in the Wedderburn decomposition of $\mathbb{R}[G]$ then $G$ is the fundamental group of a standard $\mathrm{PD}_{3}$ complex if and only if $\sigma_{4}(G)=0$ and $G$ has a balanced presentation. The condition on quaternion factors is equivalent to each of the conditions "all stably free $\mathbb{Z}[G]$-modules are free" and " $G$ has no quotient which is a quaternionic group $Q_{4 n}$ with $n \geqslant 6$ ", and the families of such groups are known [114].

It is unknown whether all finite groups of cohomological period 4 have balanced presentations. The groups $Q(8 k), T_{k}^{*}$ and $I^{*}$ have balanced 2-generator presentations [Hi, Chapter 11.2], as do the groups $Q(8, p, q)$ (with $p, q$ distinct odd primes) [Jo, page 249].

With the exception of the groups $A(m, 1)$ (with $m>1$ ), $O_{k}^{*}$ (with $k>1$ ), $Q\left(2^{n} a, b, c\right)$ (with either $n=3$ and at most one of $a, b$ and $c$ being 1 or $n>3$ and $b c>1$ ) and $P_{48 \cdot 3^{k-1} a}^{\prime \prime}$ (with $a k>1$ and $(a, 6)=1$ ), and their products with cyclic groups, all finite groups with cohomological period dividing 4 have fixed point free representations in $\mathrm{SO}(4)$, and so act freely on $S^{3}$. We shall call such groups $\mathbb{S}^{3}$-groups. (See [2] for a largely self-contained account.) Cyclic groups, the binary dihedral groups $D_{4 m}^{*}=A(m, 2)$, with $m$ odd, and $D_{8 k}^{*}=Q(8 k, 1,1)$, with $k \geqslant 1$ and the three binary polyhedral groups $T_{1}^{*}, O_{1}^{*}$ and $I^{*}$ are subgroups of $S^{3}$.

Although it is now known that the $\mathbb{S}^{3}$-groups are the only finite groups which act freely on $S^{3}$, the corresponding question for free actions on homology 3 -spheres remains open. Finite groups with such actions also have cohomological period 4. Dihedral groups $D_{2 m}$, the groups $Q\left(2^{k} a, b, c\right)$ with $k>3$ and $b c>1$, and $P_{48 a}^{\prime \prime}$ with $a>1$ and $(a, 6)=1$ have no such actions, by a surgery semicharacteristic argument [103]. The unsettled cases involve the groups $Q(8 a, b, c) \times \mathbb{Z} / d \mathbb{Z}$, with $a b c d$ odd and $b c>1$. (See [25].)

Do any of the other $\mathrm{PD}_{3}$-complexes $P$ with $\pi=\pi_{1}(P)$ finite arise in some natural context? For instance, is $P \times S^{1}$ homotopy equivalent to a closed 4-manifold? (Since the group of self-homotopy equivalences of such a complex is finite it is equivalent to ask whether there is a closed 4-manifold $M$ with $\chi(M)=0$ and $\pi_{1}(M) \cong \pi \rtimes \mathbb{Z}\left[\mathrm{Hi}\right.$, Theorem 4.7].) The case when $\pi \cong D_{2 m}$ may be ruled out by a surgery semicharacteristic argument [52].

\subsection{Fundamental group with two ends}

A group $G$ has two ends if and only if it has a finite normal subgroup $F$ such that $G / F$ is isomorphic to $\mathbb{Z}$ or $D_{\infty}$. From the cohomological perspective, $G$ has two ends if and only if $E(G) \cong \mathbb{Z}$. 
Theorem 5.8 [150]. Let $P$ be a $\mathrm{PD}_{3}$-complex such that $\pi=\pi_{1}(P)$ has two ends. Then $P$ is homotopy equivalent to one of the four $\mathbb{S}^{2} \times \mathbb{E}^{1}$-manifolds $S^{2} \times S^{1}, S^{2} \widetilde{\times} S^{1}, R P^{2} \times S^{1}$ or $R P^{3} \sharp R P^{3}$.

Proof. Since $\pi$ has two ends, $\pi_{2}(P) \cong \overline{E(\pi)}=\mathbb{Z}$, by the Hurewicz theorem and Poincare duality. The inclusion of a generator $S^{2} \rightarrow \widetilde{P}$ induces isomorphisms on homology, and so is a homotopy equivalence. If $g \in \pi$ has finite order $>1$ then $H_{2}\left(\langle g\rangle ; \pi_{2}(P)\right) \neq 0$, by Lemma 4.6. Hence $g$ acts nontrivially on $\pi_{2}(P)$, and so $g$ has order 2 . Since the subgroup of $\pi$ which acts trivially on $\pi_{2}(P)$ has index $\leqslant 2$, it follows that $\pi$ is isomorphic to $\mathbb{Z}, \mathbb{Z} / 2 \mathbb{Z} \oplus \mathbb{Z}$ or $D_{\infty}$.

If $\pi \cong \mathbb{Z}$ then, viewing $c_{P}: P \rightarrow S^{1}=K(\mathbb{Z}, 1)$ as a fibration, we see that $P \simeq S^{2} \times S^{1}$ if the action is trivial, and $P \simeq S^{2} \widetilde{\times} S^{1}$ otherwise. If $\pi \cong \mathbb{Z} / 2 \mathbb{Z} \oplus \mathbb{Z}$ then $P$ has an infinite cyclic covering $\simeq R P^{2}$. Hence $P \simeq R P^{2} \times S^{1}$, since every self homotopy equivalence of $R P^{2}$ is homotopic to the identity.

If $\pi \cong D_{\infty}$ then it is generated by elements $u, v$ of order 2 . These act nontrivially on $\Pi=\pi_{2}(P) \cong \mathbb{Z}$, and so $H^{3}(\pi ; \Pi) \cong(\mathbb{Z} / 2 \mathbb{Z})^{2}$. If $g=u$ or $v$ then $P_{\langle g\rangle} \simeq R P^{2}$, and $k_{1}\left(R P^{2}\right) \neq 0$. Therefore $k_{1}(P)$ restricts nontrivially to each of the summands $H^{3}(\langle g\rangle ; \Pi) \cong \mathbb{Z} / 2 \mathbb{Z}$ corresponding to $g=u$ or $v$, and so is uniquely determined. Hence $P \simeq R P^{3} \sharp R P^{3}$, by the argument of $\S 3$ of Chapter 2. (This case also follows from the Splitting Theorem and Theorem 5.2.) 


\section{CHAPTER 6}

\section{Indecomposable orientable $\mathrm{PD}_{3}$-complexes}

We begin this chapter with an explicit description of a finite $\mathrm{PD}_{3}$-complex $Y$ realizing the group $\pi=$ $S_{3} * \mathbb{Z} / 2 \mathbb{Z} S_{3}$, building upon the construction of Chapter 5 . This illustrates a further divergence from the known properties of 3-manifolds. In particular, the Sphere Theorem does not extend to all $\mathrm{PD}_{3}$ complexes. In $\S 2$ we show that there are just two homotopy types of $\mathrm{PD}_{3}$-complexes with this group, and each is orientable. In the rest of the chapter we use the Normalizer and Centralizer conditions to analyze the indecomposable, virtually free groups which are fundamental groups of orientable $\mathrm{PD}_{3}$ complexes. Our main result is that if $\pi$ is such a group then $\pi \cong \pi \mathcal{G}$, where $(\mathcal{G}, \Gamma)$ is a finite graph of finite groups, in which $\Gamma$ is a tree, at most one edge group is not $\mathbb{Z} / 2 \mathbb{Z}$, and at most two vertex groups are not dihedral. This chapter is based on $[65 ; 67 ; 68]$.

\subsection{A finite complex with group $S_{3} *_{\mathbb{Z} / 2 \mathbb{Z}} S_{3}$}

Let $\pi=S_{3} * \mathbb{Z} / 2 \mathbb{Z} S_{3}$, with presentation $\langle a, b, c \mid r, s, t\rangle$, where $r=a^{2}, s=a b a b^{-2}$ and $t=a c a c^{-2}$. The two obvious embeddings of $S_{3}$ into $\pi$ admit retractions, as $\pi /\langle b b\rangle \cong \pi /\langle c\rangle \cong S_{3}$. Let $A, B$ and $C$ be the cyclic subgroups generated by the images of $a, b$ and $c$, respectively. The inclusions of $A$ into $S_{3}$ and $\pi$ induce isomorphisms on abelianization, while the commutator subgroups are $S_{3}^{\prime}=B$ and $\pi^{\prime}=B * C$. Thus these groups are semidirect products:

$$
S_{3} \cong B \rtimes \mathbb{Z} / 2 \mathbb{Z} \quad \text { and } \quad \pi \cong(B * C) \rtimes \mathbb{Z} / 2 \mathbb{Z} .
$$

In particular, $\pi$ is virtually free, and has infinitely many ends. However, it follows easily from the Grushko-Neumann Theorem that $\pi$ is indecomposable.

The above presentations determine finite 2-complexes $K$ and $L$, with fundamental groups $S_{3}$ and $\pi$, respectively. There are two obvious embeddings of $K$ as a retract in $L$, with retractions $r_{b}, r_{c}: L \rightarrow K$ given by collapsing the pair of cells $\{c, t\}$ and $\{b, s\}$, respectively.

Let $\Pi=\mathbb{Z}[\pi]$. The cellular chain complex for the universal covering space $\widetilde{L}$ has the form

$$
\Pi^{3} \stackrel{\partial_{2}}{\longrightarrow} \Pi^{3} \stackrel{\partial_{1}}{\longrightarrow} \Pi .
$$

The differentials are given by

$$
\begin{array}{ll}
\partial_{1}(1,0,0)=a-1, & \partial_{2}(1,0,0)=(a+1,0,0), \\
\partial_{1}(0,1,0)=b-1, & \partial_{2}(0,1,0)=(a b+1, a-b-1,0), \\
\partial_{1}(0,0,1)=c-1, & \partial_{2}(0,0,1)=(a c+1,0, a-c-1) .
\end{array}
$$

In particular, $\operatorname{Ker}\left(\partial_{2}\right)=H_{2}(\widetilde{L} ; \mathbb{Z})$ and $\operatorname{Coker}\left(\partial_{2}\right) \cong I(\pi)$. 
Let $\theta=\left(a-1,-b a+a+b^{2}-b,-c a+a+c^{2}-c\right)$. Then $\partial_{2}(\theta)=0$, and so $\theta$ determines an element of $\pi_{2}(L)=H_{2}(\widetilde{L} ; \mathbb{Z})$, by the Hurewicz Theorem. Let $Y=L \cup_{\theta} e^{3}$ and let $D_{*}$ be the cellular chain complex for the universal covering space $\widetilde{Y}$.

We define elements of $\Pi^{3}$ as in the left column, and compute some of their images under $\partial_{1}$ and $\partial_{2}$ :

$$
\begin{array}{lll}
\tilde{e}_{1}=(1,0,0) & \Rightarrow & \partial_{1} \tilde{e}_{1}=a-1, \\
\tilde{e}_{2}=\left(-b a-b^{2}, 1,0\right) & \Rightarrow & \partial_{1} \tilde{e}_{2}=b a-a b+b^{2}-1, \\
\tilde{e}_{3}=\left(-c a-c^{2}, 0,1\right) & \Rightarrow & \partial_{1} \tilde{e}_{3}=c a-a c+c^{2}-1, \\
\tilde{f}_{1}=(1,0,0) & \Rightarrow & \partial_{2} \tilde{f}_{1}=(a+1) \tilde{e}_{1}, \\
\tilde{f}_{2}=(0,-a, 0) & \Rightarrow & \partial_{2} \tilde{f}_{2}=(a b+a-1) \tilde{e}_{2}, \\
\tilde{f}_{3}=(0,0,-a) & \Rightarrow & \partial_{2} \tilde{f}_{3}=(a c+a-1) \tilde{e}_{3} .
\end{array}
$$

Moreover $\theta=(a-1) \tilde{f}_{1}+(-a b+b a+b-1) \tilde{f}_{2}+(-a c+c a+c-1) \tilde{f}_{3}$. Let $D^{*}=\operatorname{Hom}_{\Gamma}\left(D_{*}, \Pi\right)$ be the cochain complex dual to $D_{*}$. Then it is easily seen that $\overline{D^{*}} \cong D_{3-*}$.

Theorem 6.1. The complex $Y$ is a $\mathrm{PD}_{3}$-complex.

Proof. Clearly $H_{0}\left(D_{*}\right) \cong \mathbb{Z}$ and $H_{1}\left(D_{*}\right)=0$. The argument of the first part of Lemma 5.6 extends immediately to show that the kernel of $\partial_{2}$ is generated by

$$
(a-1) \tilde{f}_{1}, \quad(b-1)(b a-1) \tilde{f}_{2} \text { and } \quad(c-1)(c a-1) \tilde{f}_{3} .
$$

Hence these elements represent generators for $H_{2}\left(D_{*}\right)$. Let $\tilde{g}$ be the generator for $D_{3}$ corresponding to the top cell, so that $\partial_{3} \tilde{g}=\theta$. Note that the image of $g$ in $\mathbb{Z} \otimes_{\varepsilon} D_{3}$ is a cycle, and represents a generator for $H_{3}(Y ; \mathbb{Z})=H_{3}\left(\mathbb{Z} \otimes_{\varepsilon} D_{*}\right)$. If $h \theta=0$ then (as in Lemma 5.6) $h=h_{1}(a+1)$ for some $h_{1} \in \mathbb{Z}[B * C]$ such that $h_{1}(b-1)=h_{1}(c-1)=0$. It follows that $h_{1}=0$. Hence $\partial_{3}$ is injective and so $H_{3}\left(D_{*}\right)=0$.

Let $\hat{g}$ be the generator of $D^{3}$ such that $\hat{g}(\tilde{g})=1$. Let $\Delta$ be a diagonal approximation for $D_{*}$ and suppose that $\Delta(\tilde{g})=\sum_{0 \leqslant q \leqslant 3} \sum_{i \in I(q)} x_{i} \otimes y_{i}$, where $x_{i} \in D_{q}$ and $y_{i} \in D_{3-q}$, for all $i \in I(q)$ and $0 \leqslant q \leqslant 3$. Then $\sum_{i \in I(3)} x_{i}=\tilde{g}$. Let $r_{i}=\hat{g}\left(x_{i}\right)$ for $i \in I(3)$ and let $\tilde{\xi}$ denote the image of $\tilde{g}$ in $H_{3}(Y ; \mathbb{Z})=\mathbb{Z} \otimes_{\varepsilon} D_{3}$. Then

$$
\varepsilon(\hat{g} \cap \tilde{\xi})=\varepsilon\left(\sum_{i \in I(3)} \overline{r_{i}} y_{i}\right)=\varepsilon\left(\sum_{i \in I(3)} \overline{r_{i}}\right)=\varepsilon(\overline{\hat{g}(\tilde{g})})=1,
$$

and so $\hat{g} \cap \tilde{\xi}$ generates $H_{0}\left(D_{*}\right)$. Since $H_{1}\left(D_{*}\right)=H_{3}\left(D_{*}\right)=H^{0}\left(\overline{D^{*}}\right)=H^{2}\left(\overline{D^{*}}\right)=0$, the map $-\cap \tilde{\xi}$ induces isomorphisms $H^{q}\left(\overline{D^{*}}\right) \cong H_{3-q}\left(D_{*}\right)$ for all $q \neq 1$. The remaining case follows as in [147] from the facts that $\overline{D^{*}} \cong D_{3-*}$ and $\Delta$ is chain homotopic to $\tau \Delta$, where $\tau: D_{*} \otimes D_{*} \rightarrow D_{*} \otimes D_{*}$ is the transposition defined by $\tau(\alpha \otimes \omega)=(-1)^{p q} \omega \otimes \alpha$ for all $\alpha \in D_{p}$ and $\omega \in D_{q}$. Thus $Y$ is a $\mathrm{PD}_{3}$-complex.

Can the last step of this argument be made more explicit? The work of Handel [53] on diagonal approximations for dihedral groups may be adapted to give the following formulae for a diagonal approximation for the truncation to degrees $\leqslant 2$ of $D_{*}$ which is compatible with the above two embeddings of $K$ as a retract in $L$ :

$$
\Delta(1)=1 \otimes 1
$$


$\Delta\left(\tilde{e}_{1}\right)=\tilde{e}_{1} \otimes a+1 \otimes \tilde{e}_{1}$,

$\Delta\left(\tilde{e}_{2}\right)=\tilde{e}_{2} \otimes 1-b a \tilde{e}_{1} \otimes(b-1)-b^{2} \tilde{e}_{1} \otimes\left(b^{2} a-1\right)-(b a-b) \otimes b a \tilde{e}_{1}-\left(b^{2}-b\right) \otimes b^{2} \tilde{e}_{1}+b \otimes \tilde{e}_{2}$,

$\Delta\left(\tilde{e}_{3}\right)=\tilde{e}_{3} \otimes 1-c a \tilde{e}_{1} \otimes(c-1)-c^{2} \tilde{e}_{1} \otimes\left(c^{2} a-1\right)-(c a-c) \otimes c a \tilde{e}_{1}-\left(c^{2}-c\right) \otimes c^{2} \tilde{e}_{1}+c \otimes \tilde{e}_{3} ;$

$\Delta\left(\tilde{f}_{1}\right)=\tilde{f}_{1} \otimes 1+\tilde{e}_{1} \otimes a \tilde{e}_{1}+1 \otimes \tilde{f}_{1}$,

$\Delta\left(\tilde{f}_{2}\right)=\tilde{f}_{2} \otimes a+\left(b^{2}+b\right) \tilde{f}_{1} \otimes(a-b a)+\left(b^{2} a+b^{2}\right) \tilde{f}_{2} \otimes(a-b a)$

$+\left(\left(b a+b^{2}-1\right) \tilde{e}_{1}+\tilde{e}_{2}\right) \otimes\left(b^{2} a \tilde{e}_{1}+b a \tilde{e}_{2}\right)$

$-\left(\left(b^{2} a+1\right) \tilde{e}_{1}+b a \tilde{e}_{2}\right) \otimes\left(\left(b a+a+b^{2}+b\right) \tilde{e}_{1}+\left(b^{2} a+a\right) \tilde{e}_{2}\right)$

$-\left((a+b) \tilde{e}_{1}+b^{2} a \tilde{e}_{2}\right) \otimes\left(\left(b a+b^{2}\right) \tilde{e}_{1}+a \tilde{e}_{2}\right)-(a+1) \tilde{e}_{1} \otimes \tilde{e}_{1}$

$+(a-b) \otimes\left(b^{2}+b\right) \tilde{f}_{1}+(a-b) \otimes\left(b^{2} a+b^{2}\right) \tilde{f}_{2}+a \otimes \tilde{f}_{2}$,

$\Delta\left(\tilde{f}_{3}\right)=\tilde{f}_{3} \otimes a+\left(c^{2}+c\right) \tilde{f}_{1} \otimes(a-c a)+\left(c^{2} a+c^{2}\right) \tilde{f}_{3} \otimes(a-c a)$

$+\left(\left(c a+c^{2}-1\right) \tilde{e}_{1}+\tilde{e}_{3}\right) \otimes\left(c^{2} a \tilde{e}_{1}+c a \tilde{e}_{3}\right)$

$-\left(\left(c^{2} a+1\right) \tilde{e}_{1}+c a \tilde{e}_{3}\right) \otimes\left(\left(c a+a+c^{2}+c\right) \tilde{e}_{1}+\left(c^{2} a+a\right) \tilde{e}_{3}\right)$

$-\left((a+c) \tilde{e}_{1}+c^{2} a \tilde{e}_{3}\right) \otimes\left(\left(c a+c^{2}\right) \tilde{e}_{1}+a \tilde{e}_{3}\right)-(a+1) \tilde{e}_{1} \otimes \tilde{e}_{1}$

$+(a-c) \otimes\left(c^{2}+c\right) \tilde{f}_{1}+(a-c) \otimes\left(c^{2} a+c^{2}\right) \tilde{f}_{3}+a \otimes \tilde{f}_{3}$.

These formulae were derived from the work of Handel by using the canonical involution of $\mathbb{Z}\left[S_{3}\right]$ to switch right and left module structures and showing that $C_{*}$ is a direct summand of a truncation of the Wall-Hamada resolution for $S_{3}$. (In Handel's notation $a=y, b=x, e_{1}=c_{1}^{2}, e_{2}=-c_{1}^{1}-c_{1}^{2}(x+x y)$, $f_{1}=c_{2}^{3}, f_{2}=-c_{2}^{1} y+c_{2}^{2} x^{2}-c_{2}^{3} y$ and $g=-\left(c_{3}^{1}+c_{3}^{3}\right)(x+y)-c_{3}^{4} y$.) Handel's work also leads to a formula for $\Delta(g)$, but it is not clear what $\Delta(\tilde{g})$ should be.

\subsection{Other $\mathrm{PD}_{3}$-complexes with this group}

Having constructed one $\mathrm{PD}_{3}$-complex with group $\pi$ one may ask how many there are. Any such $\mathrm{PD}_{3}$ complex $P$ must be orientable. For let $w=w_{1}(P)$. Let $R=\mathbb{Z}\left[\pi / \pi^{\prime}\right]=\mathbb{Z}[a] /\left(a^{2}-1\right)$, and let $M$ be the matrix for the homomorphism $\partial_{2}$ with cokernel $I(\pi)$ (with respect to the standard bases) given in $\S 1$. Then

while

$$
R \otimes_{\mathbb{Z}[\pi]} I(\pi) \cong R /(a+1) \oplus(R /(a+1,3))^{2}
$$

$$
R \otimes_{\mathbb{Z}[\pi]} D(I(\pi) ; M) \cong R /(a+w(a)) \oplus(R /(a+w(a), 3))^{2} .
$$

These modules are projective homotopy equivalent, by Corollary 2.4.1, and so $w=1$.

Two such $\mathrm{PD}_{3}$-complexes $W_{1}$ and $W_{2}$ are homotopy equivalent if and only $\mu\left(W_{1}\right)$ and $\mu\left(W_{2}\right)$ agree up to sign and the action of $\operatorname{Out}(\pi)$. When $\pi=S_{3} * \mathbb{Z} / 2 \mathbb{Z} S_{3}$ we have $F^{2}(C) \cong I(\pi)$, and

$$
H_{3}(\pi ; \mathbb{Z}) \cong H_{3}\left(\pi^{\prime} ; \mathbb{Z}\right) \oplus H_{3}(\mathbb{Z} / 2 \mathbb{Z} ; \mathbb{Z}) \cong(\mathbb{Z} / 3 \mathbb{Z})^{2} \oplus(\mathbb{Z} / 2 \mathbb{Z}) .
$$

Let $Y^{\prime}$ be the double cover of $Y$, with fundamental group $\pi^{\prime} \cong(\mathbb{Z} / 3 \mathbb{Z}) *(\mathbb{Z} / 3 \mathbb{Z})$. Then $Y^{\prime}$ is a connected sum, by the Splitting Theorem, and so it is homotopy equivalent to one of the 3-manifolds $L(3,1) \sharp L(3,1)$ 
and $L(3,1) \sharp-L(3,1)$. (These may be distinguished by the torsion linking forms on their first homology groups). In particular, $\mu\left(Y^{\prime}\right)$ has nonzero entries in each summand. Since $\mu\left(Y^{\prime}\right)$ is the image of $\mu(Y)$ under the transfer to $H_{3}\left(\pi^{\prime} ; \mathbb{Z}\right) \cong(\mathbb{Z} / 3 \mathbb{Z})^{2}$, the image of $\mu(Y)$ in each $\mathbb{Z} / 3 \mathbb{Z}$-summand must be nonzero. Let $u \in H^{1}\left(Y ; \mathbb{F}_{2}\right)$ correspond to the abelianization homomorphism. Since $\beta_{2}\left(Y ; \mathbb{F}_{2}\right)=\beta_{1}\left(Y ; \mathbb{F}_{2}\right)=1=$ $\beta_{2}\left(\pi ; \mathbb{F}_{2}\right)$ we have $u^{2} \neq 0$, and so $u^{3} \neq 0$, by Poincare duality. It follows easily that the image of $\mu(Y)$ in the $\mathbb{Z} / 2 \mathbb{Z}$-summand must be nonzero also. (Note that $Y^{\prime}$ is $\mathbb{Z}_{(2)}$-homology equivalent to $S^{3}$ and so $Y$ is $\mathbb{Z}_{(2)}[\mathbb{Z} / 2 \mathbb{Z}]$-homology equivalent to $\left.R P^{3}\right)$. Since reversing the orientation of $Y$ reverses that of $Y^{\prime}$, we may conclude that there are at most two distinct homotopy types of $\mathrm{PD}_{3}$-complexes with fundamental group $\pi$, and that they may be detected by their double covers.

Let $X=K \cup_{\psi} e^{3}$ be the $\mathrm{PD}_{3}$-complex with $\pi_{1}(X) \cong S_{3}$ constructed in $\S 4$ of Chapter 5 . The retractions $r_{b}$ and $r_{c}$ of $L$ onto $K$ extend to maps $r_{b}, r_{c}: Y \rightarrow X$. These maps induce the same isomorphism $H_{3}(Y ; \mathbb{Z}) \cong H_{3}(X ; \mathbb{Z})$, and so their lifts to the double covers induce the same isomorphism $H_{3}\left(Y^{\prime} ; \mathbb{Z}\right) \rightarrow$ $H_{3}\left(X^{\prime} ; \mathbb{Z}\right)$. Hence $Y^{\prime} \simeq L(3,1) \sharp L(3,1)$, rather than $L(3,1) \sharp-L(3,1)$. If we use

$$
\xi=(a-1) \tilde{f}_{1}+\left(-b^{2} a+b a+b-1\right) \tilde{f}_{2}-\left(-c^{2} a+c a+c-1\right) \tilde{f}_{3}
$$

instead of $\theta$ (changing only the sign of the final term) then $Z=L \cup_{\xi} e^{3}$ is another $\mathrm{PD}_{3}$-complex with $\pi_{1}(Z) \cong \pi$, and a similar argument shows that the double cover is now $Z^{\prime} \simeq L(3,1) \sharp-L(3,1)$.

\subsection{Vertex groups have periodic cohomology}

In this section we shall consider orientable $\mathrm{PD}_{3}$-complexes whose fundamental groups are virtually free. A finitely generated group $G$ is virtually free if and only if $G \cong \pi \mathcal{G}$, where $(\mathcal{G}, \Gamma)$ is a finite graph of finite groups, by [DD, Theorem IV.1.6].

Lemma 6.2. Let $\pi=\pi \mathcal{G}$, where $(\mathcal{G}, \Gamma)$ is a nontrivial reduced finite graph of groups. If there is an edge $e$ with $G_{e}=1$ then either $\pi$ is a nontrivial free product or $\pi \cong \mathbb{Z}$.

Proof. If $\Gamma \backslash\{e\}$ has two components then $\pi$ is a nontrivial free product. Otherwise a maximal tree for $\Gamma \backslash\{e\}$ is also a maximal tree for $\Gamma$, and the stable letter $t_{e}$ generates a free factor of $\pi$.

Lemma 6.3. Let $\pi=\pi \mathcal{G}$, where $(\mathcal{G}, \Gamma)$ is a finite graph of finite groups and $\Gamma$ is a tree. If all the edge groups are nontrivial then $\pi$ is indecomposable.

Proof. If $\pi \cong A * B$ then $\pi$ acts without global fixed points on the Bass-Serre tree $\Upsilon$ associated to the splitting. Each finite subgroup of $\pi$ fixes a point in this tree. If $x_{o}$ and $x_{t} \in \Upsilon$ are fixed adjacent vertex groups $G_{o(e)}$ and $G_{t(e)}$ then $G_{e}$ fixes the interval $\left[x_{o}, x_{t}\right]$ joining these points. Hence $x_{o}=x_{t}$, since edgestabilizers in $\Upsilon$ are trivial. Induction on the size of $\Gamma$ now shows that $x_{o}$ is fixed by $\pi$, contradicting the first sentence of the proof.

This argument extends easily to all finite graphs of finite groups with nontrivial edge groups, but we need only the above case.

We shall use frequently the Centralizer Condition (Theorem 4.9) and the following simple lemma.

Lemma 6.4. Let $\pi=\pi \mathcal{G}$, where $(\mathcal{G}, \Gamma)$ is a finite graph of groups. If $C$ is a subgroup of an edge group $G_{e}$ with $N_{G_{e}}(C)$ properly contained in each of $N_{G_{o(e)}}(C)$ and $N_{G_{t(e)}}(C)$, then $N_{\pi}(C)$ is infinite. 
Proof. If $g_{o} \in G_{o(e)} \backslash G_{e}$ and $g_{t} \in G_{t(e)} \backslash G_{e}$ each normalize $C$, then $g_{o} g_{t}$ normalizes $C$ and has infinite order.

We shall also need the obvious fact that if $C<\pi$ is a finite subgroup and $N_{\pi}(C)$ is infinite, then so is $C_{\pi}(C)$.

Lemma 6.5. Let $X$ be an orientable $\mathrm{PD}_{3}$-complex with $\pi=\pi_{1}(X) \cong \pi \mathcal{G}$, where $(\mathcal{G}, \Gamma)$ is a reduced finite graph of finite groups. If $(\mathcal{G}, \Gamma)$ has a loop isomorphism then $\pi$ has a nontrivial free factor.

Proof. If $(\mathcal{G}, \Gamma)$ has a loop isomorphism at the edge $e$ then $t_{e}$ normalizes $G_{e}$, and so $N_{\pi}\left(G_{e}\right)$ is infinite. Therefore $G_{e}=1$, by the Centralizer Condition, and so $t_{e}$ generates a free factor of $\pi$.

If $\pi$ has a free normal subgroup $F$ of finite index then the canonical surjection $s: \pi \rightarrow G=\pi / F$ is injective on every finite subgroup of $\pi$. In particular, if $H$ is a finite subgroup of $\pi$ then the subgroup $F H=s^{-1} s(H)$ generated by $F$ and $H$ is a semidirect product $F \rtimes H$.

Lemma 6.6. Let $X$ be an indecomposable orientable $\mathrm{PD}_{3}$-complex. If $\pi=\pi_{1}(X)$ has a free normal subgroup $F$ such that $\pi / F$ is a finite nilpotent group, then $\pi$ is cyclic or $\pi \cong Q\left(2^{k}\right) \times \mathbb{Z} / d \mathbb{Z}$ for some $k \geqslant 3$ and odd $d$.

Proof. If $\pi$ has a free factor then $\pi \cong \mathbb{Z}$. Otherwise we may assume that $\pi=\pi \mathcal{G}$, where $(\mathcal{G}, \Gamma)$ is a reduced finite graph of finite groups with no loop isomorphisms. Thus each edge group $G_{e}$ is a proper subgroup of each of $G_{o(e)}$ and $G_{t(e)}$. The vertex groups are nilpotent since they map injectively to $\pi / F$. Hence the normalizer of $G_{e}$ in each of $G_{o(e)}$ and $G_{t(e)}$ is strictly larger than $G_{e}$, since nilpotent groups satisfy the Normalizer Condition. Hence $N_{\pi}\left(G_{e}\right)$ is infinite, by Lemma 6.4, and so $G_{e}=1$.

Since $X$ is indecomposable so is $\pi$, and since $\pi$ has no free factor $\Gamma$ has one vertex and no edges. Hence $\pi$ is finite, and so $\widetilde{X} \simeq S^{3}$. Therefore $\pi$ has cohomological period dividing 4. Since it is nilpotent it is cyclic or the direct product of a cyclic group of odd order with a quaternionic 2-group $Q\left(2^{k}\right)$, for some $k \geqslant 3$.

Theorem 6.7. Let $X$ be an orientable $\mathrm{PD}_{3}$-complex with $\pi=\pi_{1}(X) \cong \pi \mathcal{G}$, where $(\mathcal{G}, \Gamma)$ is a reduced finite graph of finite groups. Then the vertex groups have periodic cohomology and the edge groups are metacyclic.

Proof. Let $F$ be a maximal free normal subgroup of $\pi$. If $S$ is a Sylow $p$-subgroup of a vertex group $G_{v}$, then $F S$ is the fundamental group of a finite graph of $p$-groups. The indecomposable factors of $F S$ are either infinite cyclic or are finite and have periodic cohomology, by Lemma 6.6. Therefore $S$ has periodic cohomology. Since a finite group has periodic cohomology if and only if this holds for all its Sylow subgroups [Br, Proposition VI.9.3] , it follows that $G_{v}$ has periodic cohomology.

If $G_{e}$ is not metacyclic it has a central involution, which is a square, by Lemma 5.3. This involution is orientation-preserving, and is also central in each of $G_{o(e)}$ and $G_{t(e)}$, since they cannot be metacyclic. This contradicts the Centralizer Condition.

Corollary 6.7.1. For any edge $e$, at least one of the vertex groups $G_{o(e)}$ or $G_{t(e)}$ is metacyclic. If they are each metacyclic then $G_{e}$ is cyclic.

Proof. If neither $G_{o(e)}$ nor $G_{t(e)}$ is metacyclic then each has a central involution, $g_{o}$ and $g_{t}$, say. If $\left|G_{e}\right|$ is even then $g_{o}$ and $g_{t}$ are each in $\zeta G_{e}$, and hence are equal. But then $N_{\pi}\left(g_{o}\right)$ contains both vertex groups, 
and so is infinite. If $\left|G_{e}\right|$ is odd it is properly contained in each of its normalizers. In either case this contradicts the Centralizer Condition.

If $G_{o(e)}$ and $G_{t(e)}$ are each metacyclic then $G_{e}^{\prime}$ is normal in each of them, and so must be trivial, by the Centralizer Condition.

Corollary 6.7.2. If the orders of all the edge groups have a common prime factor $p$ then $\Gamma$ is a tree, and there is at most one vertex group $V=G_{v}$ such that $G_{e}<N_{V}\left(G_{e}\right)$ for some edge e with $v \in\{o(e), t(e)\}$.

Proof. Let $T$ be a maximal tree in $\Gamma$. If there is an edge $e$ not in $T$ there is a cycle $\gamma$ in $\Gamma$ incorporating $e$. Each vertex group $G_{v}$ has an unique conjugacy class of subgroups $C_{v}$ of order $p$, since its Sylow subgroups are cyclic or quaternionic. Therefore $t_{e} C_{o(e)} t_{e}^{-1}=w C_{o(e)} w^{-1}$, where $w$ is a word in the union of the vertex groups along the rest of the cycle. The element $t_{e} w^{-1}$ has infinite order, and so $N_{\pi}\left(C_{o(e)}\right)$ is infinite. This contradicts the Centralizer Condition.

If $G_{e}<N_{V}\left(G_{e}\right)$ for some $V=G_{v}$ with $v \in\{o(e), t(e)\}$, we may assume that $C_{v} \in G_{e}$. Then $N_{G_{e}}\left(C_{v}\right)<N_{V}\left(C_{v}\right)$, since $C_{v}$ is unique up to conjugacy in $G_{e}$. Suppose there are two such vertex groups $V=G_{v}$ and $W=G_{w}$ with $v \neq w$, and choose a (minimal) path connecting these vertices. As before, $C_{w}=a C_{v} a^{-1}$ for some $a$ in the subgroup generated by the intermediate vertex groups along the path. Thus $C_{w}$ is normalized by the subgroup generated by $N_{W}\left(C_{w}\right)$ and $a N_{V}\left(C_{v}\right) a^{-1}$, which is infinite. This again contradicts the Centralizer Condition.

\subsection{Eliminating groups with cohomological period $>4$}

The fact that the Sylow subgroups of a group $G$ have cohomological period dividing 4 does not imply that $G$ has cohomological period dividing 4. Nevertheless, this is true in our situation.

Theorem 6.8. Let $X$ be an orientable $\mathrm{PD}_{3}$-complex with $\pi=\pi_{1}(X) \cong \pi \mathcal{G}$, where $(\mathcal{G}, \Gamma)$ is a reduced finite graph of finite groups. Then the vertex groups have cohomological period dividing 4.

Proof. Let $F$ be a free normal subgroup of finite index in $\pi$. Suppose there is a vertex group with cohomological period greater than 4 . Then it has a subgroup $H \cong \mathbb{Z} / p \mathbb{Z} \rtimes \mathbb{Z} / q \mathbb{Z}$ with a presentation

$$
\left\langle a, b \mid a^{q}=b^{p}=1, a b a^{-1}=b^{r}\right\rangle,
$$

where, by Lemma 5.5, either (1) $p$ is an odd prime, $q$ is an odd prime or 4 and $r$ is a primitive $q$-th root $\bmod p$, or (2) $p=5, q=8$ and $r=2$, Let $f: \pi \rightarrow \pi / F$ be the canonical projection, and let $F H=f^{-1} f(H)$. Then $F H \cong F \rtimes H$ is the group of an orientable $\mathrm{PD}_{3}$-complex. Since every finite subgroup of a free product is conjugate to a subgroup of one of the factors, we may assume that $\pi=F H$ and is indecomposable. We may also assume that $\Gamma$ has at least one edge, for otherwise $\pi \cong H$ is finite, and so has cohomological period 4, by Theorem 5.2.

Assume first that $q$ is an odd prime. Since $\pi$ is indecomposable and all centralizers of non-identity elements are finite, we may assume that all edge groups have order $q$. Since the Sylow $q$-subgroups in each vertex group are all conjugate, we may assume also that $\Gamma$ is a tree, by Corollary 6.7.2, and that $f$ maps each vertex group isomorphically onto $H$. It follows that $\pi$ has a presentation

$$
\left\langle a, b_{1}, \ldots, b_{n} \mid a^{q}=b_{i}^{p}=1, a b_{i} a^{-1}=b_{i}^{r} \forall i\right\rangle .
$$

Let $f: \mathbb{Z}[\pi] \rightarrow R=\mathbb{Z}[\mathbb{Z} / q \mathbb{Z}]$ be the epimorphism whose kernel is the two-sided ideal generated by 
$\left\{b_{1}-1, \ldots, b_{n}-1\right\}$. The $\mathbb{Z}$-torsion of $R \otimes_{f} I(\pi)$ is $(\mathbb{Z} / p \mathbb{Z})^{n}$, with $a$ acting as multiplication by $r$, whereas $a$ acts on the $\mathbb{Z}$-torsion of $R \otimes_{f} D I(\pi)$ as multiplication by $r^{-1}$.

Let $N \cong R^{2} / R\left(p, a^{-1}-r\right)$ and $\rho=\sum a_{i<q} a^{i} r^{i}$ in $R$. Then

$$
\left(a^{-1}-r\right) \rho=a^{-1}\left(1-a^{q} r^{q}\right)=a^{-1}\left(1-r^{q}\right) \equiv 0 \bmod p .
$$

Therefore $\left(a^{-1}-r\right) \rho=p \sigma$ for some $\sigma \in R$. Let $[\rho, \sigma]$ be the image of $(\rho, \sigma)$ in $N$. Then $[\rho, \sigma] \neq 0$, since $p$ does not divide $\rho$ in $R$. On the other hand,

$$
p[\rho, \sigma]=\rho\left[p, a^{-1}-r\right] \quad \text { and } \quad\left(a^{-1}-r\right)[\rho, \sigma]=\sigma\left[p, a^{-1}-r\right]=0 .
$$

Thus $a$ acts as multiplication by $r^{-1}$ on this nontrivial $p$-torsion element of $N$. Since $R \otimes_{f} D I(\pi) \cong$ $I(\mathbb{Z} / q \mathbb{Z}) \oplus N^{n}$ and $r^{-1} \not \equiv r \bmod p$, it follows that there are no projective $\mathbb{Z}[\pi]$-modules $P, Q$ such that $R \otimes_{f}(I(\pi) \oplus P) \cong R \otimes_{f}(D I(\pi) \oplus Q)$. Thus the conclusion of Corollary 2.4.1 does not hold.

If $q=4$ the edge groups have order 2 or 4 , and at least one vertex group has an element of order 4 . We may again assume that $\Gamma$ is a tree, and $\pi$ now has a presentation of the form

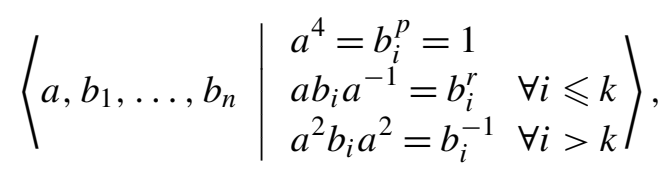

for some $k>1$. We now find that $a$ acts as multiplication by $r$ on a summand $(\mathbb{Z} / p \mathbb{Z})^{k}$ of the $\mathbb{Z}$-torsion of $R \otimes_{f} I(\pi)$, whereas it acts by $r^{-1}=-r$ on the corresponding summand of the $\mathbb{Z}$-torsion of $R \otimes_{f} D I(\pi)$. We again find that Corollary 2.4.1 does not hold.

Suppose finally that $p=5, q=8$ and $r=2$. In this case every subgroup of $H$ of order 2 or 5 is normal in $H$. Hence $\Gamma$ can have no edges, contrary to our assumption.

Thus all vertex groups have cohomological period dividing 4 .

\subsection{Groups with cohomological period 1,2 or 4}

We shall now use the classification of groups of cohomological period 4 to restrict further the possible fundamental groups.

Lemma 6.9. Let $G$ be a finite group with cohomological period 4 , and let $C$ be a cyclic subgroup of odd prime order $p$. Then $N_{G}(C)$ is nonabelian unless $p=3$ and $G=B \times \mathbb{Z} / d \mathbb{Z}$ with $B=T_{1}^{*}$ or $I^{*}$.

Proof. This follows on examining the list of such groups $G$. (Note that if $p>5$ then $C$ is central, while if $p=5$ and $G=I^{*}$ or $p=3$ and $G=O_{1}^{*}$ then $N_{G}(C)$ is nonabelian. If $p=3$ and $G=T_{k}^{*}$ or $O_{k}^{*}$ with $k>1$ then $C$ is normal in $G$.)

Theorem 6.10. Let $X$ be an indecomposable orientable $\mathrm{PD}_{3}$-complex with $\pi=\pi_{1}(X) \cong \pi \mathcal{G}$, where $(\mathcal{G}, \Gamma)$ is a reduced finite graph of finite groups of cohomological period dividing 4 . Then:

(1) $\Gamma$ is a tree and at most one edge group is not $\mathbb{Z} / 2 \mathbb{Z}$.

(2) If all edge groups are $\mathbb{Z} / 2 \mathbb{Z}$ then at most one vertex group is not dihedral.

(3) If there is an edge e with $\left|G_{e}\right|>2$, then $G_{e} \cong \mathbb{Z} / 6 \mathbb{Z}$, the adjacent vertex groups are $D_{2 m} \times \mathbb{Z} / 3 \mathbb{Z}$ and $B \times \mathbb{Z} / d \mathbb{Z}$, with $(m, 6)=1, B=T_{1}^{*}$ or $I^{*}$ and $(d,|B|)=1$, and the remaining vertex groups are dihedral. 
Proof. Let $G_{e}$ be an edge group. Then $G_{e}$ is metacyclic, by Theorem 6.7. If 4 divides $\left|G_{e}\right|$ then $G_{e}$ has a central involution, which is also central in $V=G_{o(e)}$ and $W=G_{t(e)}$, since these groups have cohomological period dividing 4. This contradicts the Centralizer Condition, and so 4 cannot divide $\left|G_{e}\right|$.

At least one of $V, W$ is metacyclic, by Corollary 6.7.1. Suppose that both are metacyclic. If $C \leqslant G_{e}$ has odd prime order then $N_{V}(C)=V$ and $N_{W}(C)=W$, since $V$ and $W$ are metacyclic with cohomological period dividing 4. As this contradicts the Centralizer Condition, $G_{e}=\mathbb{Z} / 2 \mathbb{Z}$.

If $V$ is not metacyclic then it has a central involution, $g$ say, and $W \cong D_{2 m} \times \mathbb{Z} / k \mathbb{Z}$ for some relatively prime odd $m \geqslant 3$ and $k \geqslant 1$. Therefore if $C \leqslant G_{e}$ has odd prime order $N_{W}(C)=W$. Hence $N_{V}(C) \leqslant G_{e}$ and so the central involution is in $G_{e}$. Moreover, $C_{W}(g)=G_{e}$ and so $G_{e} \cong \mathbb{Z} / 2 k \mathbb{Z}$. Since the odd-order subgroup of $G_{e}$ is central in $W$ its normalizer in $V$ must be abelian. Hence either $k=3$ and $V=B \times \mathbb{Z} / d \mathbb{Z}$, with $B=T_{1}^{*}$ or $I^{*}$ and $(d,|B|)=1$, or $k=1$, by Lemma 6.9.

Since the edge groups all have even order, and groups of cohomological period 4 and order divisible by 4 have central involutions, there is at most one such vertex group and $\Gamma$ is a tree, by Corollary 6.7.2.

If $\mathbb{Z} / 6 \mathbb{Z}$ is an edge group then some subgroup $\rho$ of finite index in $\pi$ has a reduced graph of groups structure with a vertex group $T_{1}^{*}$ and an edge group $\mathbb{Z} / 6 \mathbb{Z}$. Factoring out the commutator subgroups of the dihedral vertex groups gives a ring epimorphism $f: \mathbb{Z}[\rho] \rightarrow \mathbb{Z}[\sigma]$, where $\sigma=\left(D_{2 m} \times \mathbb{Z} / 3 \mathbb{Z}\right) * \mathbb{Z} / 6 \mathbb{Z} T_{1}^{*}$. (This group has the presentation $\left\langle w, x, z \mid z^{2} x=x z x z, x^{2}=z^{3}, w z w=z, z^{m}=1\right\rangle$.) We may use $f$ to show that if $\rho$ satisfies the Turaev criterion then so does $\sigma$. We know of no such examples, but think a new idea may be needed to apply the Turaev criterion effectively in this case.

Since all involutions in $\pi$ are conjugate we may modify $(\mathcal{G}, \Gamma)$ so that $\Gamma$ is linear: all vertices have valence $\leqslant 2$.

Corollary 6.10.1. If all the vertex groups are dihedral then $\pi \cong \pi^{\prime} \rtimes \mathbb{Z} / 2 \mathbb{Z}$ and $\pi^{\prime}$ is a free product of cyclic groups of odd order.

Corollary 6.10.2. The group $\pi$ retracts onto one of its vertex groups, and the retraction induces isomorphisms on homology with simple coefficients.

Proof. Suppose that the vertex groups are $\left\{G_{1}, \ldots, G_{n}\right\}$, where $G_{i}$ is dihedral for $i \leqslant n-2$ and $G_{n-1}$ is dihedral or $D_{2 m} \times \mathbb{Z} / 3 \mathbb{Z}$. Then $\pi /\left\langle\left\langle\bigcup_{i<n} G_{i}^{\prime}\right\rangle\right\rangle \cong G_{n}$.

The second assertion follows by a simple Mayer-Vietoris argument.

Theorem 6.10 and Milnor's 2-Torsion Central Theorem [110] (on involutions in finite groups acting freely on mod-(2) homology spheres - see $\$ 3$ of Chapter 5) together imply (without using the Sphere Theorem) that if $M$ is a closed orientable 3-manifold and $\pi=\pi_{1}(M)$ is freely indecomposable, then $\pi$ is a finite group, $\mathbb{Z}, \mathbb{Z} \oplus(\mathbb{Z} / 2 \mathbb{Z})$, or a $\mathrm{PD}_{3}$-group. For otherwise $\pi$ would have a finite index subgroup $v \cong\left(*_{i \leqslant r} \mathbb{Z} / m_{i} \mathbb{Z}\right) \rtimes \mathbb{Z} / 2 \mathbb{Z}$, with $m_{i}$ odd for $i \leqslant r$, by Theorem 6.10. Such a group $v$ maps onto $D_{2 m_{1}}$ with kernel $\kappa$ a free product of finite cyclic groups of odd order. Thus $D_{2 m_{1}}$ would act freely on the covering space $M_{\kappa}$ associated to $\kappa$, which is a mod-(2) homology 3-sphere. This is impossible, by the 2-Torsion Central Theorem. 


\subsection{Extending the construction of $\S 1$}

The Fox-Lyndon presentation matrix for the augmentation ideal of $D_{4 s+2}$ derived from the presentation $\left\langle a, b \mid a^{2}=1, a b^{s} a=b^{s+1}\right\rangle$ is $\left(\begin{array}{cc}a+1 & 0 \\ 1+a b^{s} & a v_{s}-v_{s+1}\end{array}\right)$, where $v_{k}=1+b+\cdots+b^{k-1}$. The off-diagonal element may be removed by right multiplication by $\left(\begin{array}{cc}1 & 0 \\ 1+a b^{s} & 1\end{array}\right)$, since

$$
\left(1+a b^{s}\right)+\left(a v_{s}-v_{s+1}\right)\left(1+a b^{s}\right)=0 .
$$

On multiplying the second column by $b^{s^{2}}$ the entries become self-conjugate.

Let $\left\{G_{i} \mid 0 \leqslant i \leqslant n\right\}$ be a family of finite groups, with $G_{0}$ having even order and cohomological period 2 or 4 , and $G_{i}=D_{2 m_{i}}$ being dihedral, with $m_{i}=2 s_{i}+1$, for $i \geqslant 1$. Each of these groups has an unique conjugacy class of involutions, and so there is a well-defined iterated generalized free product with amalgamation

$$
\pi=G_{0} * \mathbb{Z} / 2 \mathbb{Z} G_{1} * \mathbb{Z} / 2 \mathbb{Z} \cdots * \mathbb{Z} / 2 \mathbb{Z} G_{n} .
$$

We may choose a presentation for $G_{0}$ with $g$ generators and $h$ relators, in which the last generator, $a$ say, is an involution. Taking 2-generator presentations for the dihedral groups, as above, and identifying the involutions, we obtain a presentation for $\pi$ of the form

$$
\left\langle G_{0}, b_{1}, \ldots, b_{n} \mid a b_{1}^{s_{1}} a b_{1}^{-1-s_{1}}=\cdots=a b_{n}^{s_{n}} a b_{n}^{-1-s_{n}}=1\right\rangle .
$$

The corresponding Fox-Lyndon presentation matrix for $I(\pi)$ begins with an $h \times g$ block corresponding to the presentation matrix for $I_{G_{0}}$ and $n$ new rows and columns. The final $n$ elements of the $g$-th column may be removed and the diagonal elements rendered self-conjugate, as before, as the new generators interact only with $a$. (Note that if $e_{1}, \ldots, e_{g+n}$ are the generators for $I_{\pi}$ associated to this presentation then $(a+1) e_{g}=0$ is a consequence of the first $h$ relations.)

It is now clear that $[I(\pi)]=[D I(\pi)]$, and so $\pi$ is the fundamental group of a $\mathrm{PD}_{3}$-complex. If $I\left(G_{0}\right)$ has a square presentation matrix which is conjugate to its transpose, the construction of $\S 1$ extends to give an explicit complex with one 0-cell, $g+n$ 1-cells, $g+n$ 2-cells and one 3 -cell realizing this group. That this complex is a $\mathrm{PD}_{3}$-complex follows from Theorem 2.1.

The first such group considered in this context was $S_{3} * \mathbb{Z} / 2 \mathbb{Z} S_{3}[61 ; 64 ; 67]$, but the simplest such example is perhaps $S_{3} * \mathbb{Z} / 2 \mathbb{Z} \mathbb{Z} / 4 \mathbb{Z}$, with presentation

$$
\left\langle a, b \mid a^{4}=1, a^{2} b a^{2}=b^{2}\right\rangle .
$$

This group is realized by a $\mathrm{PD}_{3}$-complex with just six cells.

\subsection{Homotopy types with given fundamental group}

Let $W$ be an orientable $\mathrm{PD}_{3}$-complex with fundamental group $\pi$ and fundamental class $[W] \in H_{3}(W ; \mathbb{Z})$. Let $\mu(W)=c_{W *}[W] \in H_{3}(\pi ; \mathbb{Z})$. If $\pi$ is virtually free then $H_{3}(\pi ; \mathbb{Z})$ is finite, and so there are only finitely many homological triples, and hence homotopy types, with group $\pi$. Note also that if $\pi$ is indecomposable and virtually free then $\operatorname{Out}(\pi)$ is finite [18], and so the group of self-homotopy equivalences of $W$ is finite [57].

Suppose that $\pi=G_{0} * \mathbb{Z} / 2 \mathbb{Z} \rho$, where $G_{0}$ has cohomological period dividing 4 and a central involution and $\rho$ is an iterated free product of dihedral groups $G_{i}=D_{2 m_{i}}$ with amalgamation over copies of $\mathbb{Z} / 2 \mathbb{Z}$, 
where $m_{i}=2 s_{i}+1$, for $i \leqslant n$. Then $\rho^{\prime} \cong *_{i=1}^{n} \mathbb{Z} / m_{i} \mathbb{Z}$. Let $m_{0}=\left|G_{0}\right|$. (We allow the possibility $G_{0}=\mathbb{Z} / 2 \mathbb{Z}$.) Since $\rho \cong \rho^{\prime} \rtimes \mathbb{Z} / 2 \mathbb{Z}$ we have

$$
H_{3}(\rho ; \mathbb{Z}) \cong H_{3}(\mathbb{Z} / 2 \mathbb{Z} ; \mathbb{Z}) \oplus H_{3}\left(\rho^{\prime} ; \mathbb{Z}\right) .
$$

A Mayer-Vietoris argument then gives

$$
H_{3}(\pi ; \mathbb{Z}) \cong H_{3}\left(G_{0} ; \mathbb{Z}\right) \oplus H_{3}\left(\rho^{\prime} ; \mathbb{Z}\right)=\bigoplus_{i=0}^{n}\left(\mathbb{Z} / m_{i} \mathbb{Z}\right) .
$$

Let $f: \pi \rightarrow G_{0}$ be the epimorphism with kernel normally generated by $\rho^{\prime}$, and let $W_{\sigma}$ be the covering space corresponding to $\sigma=f^{-1}(S)$, where $S<G_{0}$ is a Sylow $p$-subgroup of $G_{0}$. If $p$ is odd, $W_{\sigma}$ is a connected sum of lens spaces, by the Splitting Theorem. Since $\mu\left(W_{\sigma}\right)$ is the image of $\mu(W)$ under transfer, it follows that $\mu(W)$ must project to a generator of each of the odd cyclic summands of $H_{3}(\pi ; \mathbb{Z})$. If $p=2$ we may argue instead that the square $\mathrm{Sq}^{1}: H^{1}\left(W_{\sigma} ; \mathbb{F}_{2}\right) \rightarrow H^{2}\left(W_{\sigma} ; \mathbb{F}_{2}\right)$ is nonzero. Hence the generator of $H^{3}\left(W_{\sigma} ; \mathbb{F}_{2}\right)$ is a product of elements in the image of $H^{1}\left(\sigma ; \mathbb{F}_{2}\right)$, by Poincare duality. It follows that the image of $\mu(W)$ in the 2-primary summand must generate also.

For each $1 \leqslant i \leqslant n$ and $u \in\left(\mathbb{Z} / m_{i} \mathbb{Z}\right)^{\times}$there is an automorphism which sends $b_{i}$ to $b_{i}^{u}$, for $b_{i} \in G_{i}^{\prime}$, and which fixes the other vertex groups. If $G_{i} \cong G_{j}$ there is an automorphism interchanging $G_{i}$ and $G_{j}$. As every automorphism of $G_{0}$ fixes the central involution it extends to an automorphism of $\pi$ which fixes $\rho$. These automorphisms act naturally on $H_{3}(\pi ; \mathbb{Z})$.

In particular, if $G_{0}=\mathbb{Z} / 2 \mathbb{Z}$, so $\pi \cong \pi^{\prime} \rtimes \mathbb{Z} / 2 \mathbb{Z}$, the double cover $W^{\prime}$ is a connected sum of lens spaces. Taking into account the actions of these automorphisms and the homotopy classification of lens spaces, we see that $W_{1} \simeq W_{2}$ if and only if $W_{1}^{\prime} \simeq W_{2}^{\prime}$. 


\section{CHAPTER 7}

\section{Nonorientable $\mathrm{PD}_{3}$-complexes}

In this chapter we turn to the nonorientable case, where the situation is somewhat different. We begin by showing that if $X$ is an indecomposable $\mathrm{PD}_{3}$-complex which is not orientable and $\pi=\pi_{1}(X)$ is virtually free, then $X$ is homotopy equivalent to $S^{2} \widetilde{\times} S^{1}$ or to $R P^{2} \times S^{1}$, and so $\pi$ is isomorphic to $\mathbb{Z}$ or to $\mathbb{Z} / 2 \mathbb{Z} \oplus \mathbb{Z}$. In particular, $\pi^{+}$is torsion-free. We then show that this remains true if $\pi$ is not virtually free. As a warm-up, we give a short proof for the case of 3-manifolds, based on the "projective plane theorem" of [36]. (The fact that $R P^{2}$ does not bound provides a further restriction in the 3-manifold case which is not yet known in general.) Our main result is Theorem 7.10, in which we show that if $P$ is an indecomposable, nonorientable $\mathrm{PD}_{3}$-complex such that $\pi=\pi_{1}(P)$ has infinitely many ends, then $\pi^{+}$is torsion-free, but not free, and $\pi \cong \pi^{+} \rtimes \mathbb{Z} / 2 \mathbb{Z}^{-}$. By passing to Sylow subgroups of the torsion in $\pi$, we may reduce potential counter-examples to special cases, which are eliminated by Lemmas 7.7, 7.8 and 7.9. This chapter is based on [68;69]; the arguments are similar to those of Chapter 6.

\subsection{Nonorientable $\mathrm{PD}_{3}$-complexes with $\pi$ virtually free}

Here we shall show that the only indecomposable nonorientable $\mathrm{PD}_{3}$-complexes with virtually free fundamental group are the two 3-manifolds $S^{2} \widetilde{\sim} S^{1}$ and $R P^{2} \times S^{1}$.

Theorem 7.1. Let $P$ be an indecomposable nonorientable $\mathrm{PD}_{3}$-complex with $\pi=\pi_{1}(P) \cong \pi \mathcal{G}$, where $(\mathcal{G}, \Gamma)$ is a finite graph of finite groups. If all the vertex groups are orientation-preserving then $P \simeq$ $S^{2} \tilde{\times} S^{1}$.

Proof. If some edge group is nontrivial then all are nontrivial, since $\pi$ is indecomposable. As they are orientation-preserving, they are also edge groups for a corresponding graph-of-groups structure for $\pi^{+}$, and so have even order, by Theorem 6.10. But then $\Gamma$ would be a tree, as in Corollary 6.7.2, and $P$ would be orientable. Thus $\pi$ must be a free group. Since it is nontrivial and indecomposable it must be $\mathbb{Z}$. The result now follows from Theorem 5.8.

When there is an orientation-reversing element of finite order the argumnent is more involved, and we need two preparatory lemmas.

Lemma 7.2. Set $R=\mathbb{Z}[\mathbb{Z} / 2 \mathbb{Z}]=\mathbb{Z}[a] /\left(a^{2}-1\right)$. Let $\pi$ be a finitely presentable group, $w: \pi \rightarrow \mathbb{Z} / 2 \mathbb{Z}$ an epimorphism, and $f: \mathbb{Z}[\pi] \rightarrow R$ the epimorphism of rings induced by $w$. Suppose that $R \otimes_{f} I(\pi) \cong$ $R /(a+1) \oplus T$, where $T$ is a $\mathbb{Z}$-torsion module. Then $[I(\pi)]_{\mathrm{pr}} \neq[D I(\pi)]_{\mathrm{pr}}$.

Proof. Every finitely generated $\mathbb{Z}$-torsion-free $R$-module is a direct sum of copies of $R, \mathbb{Z}=R /(a-1)$ and $\mathbb{Z}^{w}=R /(a+1)$, and the number of summands of each type is uniquely determined [CR, Theorem 74.3]. In particular, all finitely generated projective $R$-modules are free, and so the numbers of summands of types $\mathbb{Z}$ and $\mathbb{Z}^{w}$ are invariant under stabilization. 
Let $P$ be a presentation matrix for $T$. Then $A=\left(\begin{array}{cc}a+1 & 0 \\ 0 & P\end{array}\right)$ is a presentation matrix for $R \otimes_{f} I(\pi)$. The stable isomorphism class $\left[R \otimes_{f} D I(\pi)\right]$ contains the module presentated by $\bar{A}^{\mathrm{tr}}=\left(\begin{array}{ccc}1-a & 0 \\ 0 & \frac{\mathscr{P}^{\mathrm{tr}}}{}\end{array}\right)$. This has $\mathbb{Z}$ as a direct summand, whereas $R \otimes_{f} I(\pi)$ does not. Therefore the conclusion of Corollary 2.4.1 does not hold, and so $[I(\pi)]_{\mathrm{pr}} \neq[D I(\pi)]_{\mathrm{pr}}$.

Lemma 7.3. Let $P$ be an indecomposable $\mathrm{PD}_{3}$-complex with $\pi=\pi_{1}(P) \cong F(r) \rtimes G$, where $G$ is finite. If $\pi$ has an orientation-reversing element $g$ of finite order then it has an orientation-reversing element of order 2, and $G$ has order $2 m$, for some odd $m$.

Proof. If an orientation-reversing element $g$ has order $2^{k} d$ with $d$ odd then $k \geqslant 1$ and $g^{d}$ is orientationreversing and of order $2^{k}$. Suppose that $k>1$. After passing to an indecomposable factor of a finite covering space, if necessary, we may assume that $G$ is generated by $g^{d}$, and the graph of groups is reduced. Hence $G$ is cyclic of order $2^{k}$, while $\pi$ is indecomposable and infinite. The vertex groups are cyclic of order dividing $2^{k}$, and $\Gamma$ has at least one edge $e$. The edge group $G_{e}$ must be $\mathbb{Z} / 2 \mathbb{Z}^{-}$by the Normalizer and Centralizer Conditions. Since the inclusion of $G_{e}$ splits $w$, it follows that $k=1$. Thus $g^{d}$ is an orientation-reversing element of order 2.

Suppose that $|G|$ is a multiple of 4 . We may assume now that $G$ is a 2-group, $\pi$ is indecomposable and the graph of groups is reduced. The edge groups must be generated by orientation-reversing elements of order 2 , and the vertex groups must have order 4 . The vertex groups are $\mathbb{Z} / 2 \mathbb{Z} \oplus \mathbb{Z} / 2 \mathbb{Z}^{-}$, since the inclusion of an edge group splits $w$. (Thus $k=1$ and each vertex group has two conjugacy classes of orientation-reversing elements of order 2.)

All vertices of $\Gamma$ have valency at most 2 , for otherwise there would be an orientation-reversing involution with centralizer containing $(\mathbb{Z} / 2 \mathbb{Z}) *(\mathbb{Z} / 2 \mathbb{Z}) *(\mathbb{Z} / 2 \mathbb{Z})$. Thus either $\Gamma$ is a tree or $\beta_{1}(\Gamma)=1$.

Let $w=w_{1}(X)$ and let $f: \mathbb{Z}[\pi] \rightarrow R=\mathbb{Z}[\mathbb{Z} / 2 \mathbb{Z}]=\mathbb{Z}[a] /\left(a^{2}-1\right)$ be the epimorphism induced by $w$. Then $f$ induces an epimorphism from $I_{\pi}$ to $I_{\mathbb{Z} / 2 \mathbb{Z}}=R /(a+1)$, which factors through an epimorphism $h: R \otimes_{f} I_{\pi} \rightarrow R /(a+1)$. The inclusion of an edge group splits $h$, and so $R \otimes_{f} I_{\pi} \cong R /(a+1) \oplus N$, where $N=\operatorname{Ker}(h)$.

If $\Gamma$ is a tree then $\pi$ has a presentation

$$
\left\langle\begin{array}{l|l}
a_{1}, \ldots, a_{n}, b_{1}, \ldots, b_{n} \mid \begin{array}{ll}
a_{i}^{2}=b_{i}^{2}=a_{i} b_{i} a_{i}^{-1} b_{i}^{-1}=1 & \forall i \leqslant n \\
a_{i}=a_{i+1} b_{i+1} & \forall 1 \leqslant i<n
\end{array}
\end{array}\right\},
$$

where $w\left(a_{i}\right)=-1$ and $w\left(b_{i}\right)=1$ for all $i \leqslant n$. (The amalgamations must be essentially as in the final set of relations since the edge groups are generated by orientation-reversing involutions and each of the edge group centralizers has two ends.) Consideration of the Fox-Lyndon presentation matrix for $R \otimes_{f} I_{\pi}$ shows that $\mathbb{Q} \otimes_{\mathbb{Z}} N=0$ in this case. Thus $N$ is a $\mathbb{Z}$-torsion module, so $\left[I_{\pi}\right] \neq\left[J_{\pi}\right]$, by Lemma 7.2. Therefore $\Gamma$ cannot be a tree.

If $\beta_{1}(\Gamma)=1$ then $\pi$ has a presentation

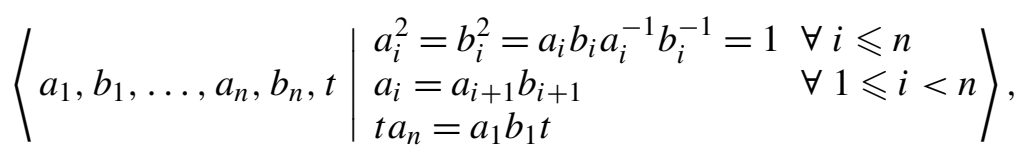

where $w\left(a_{i}\right)=-1$ and $w\left(b_{i}\right)=1$ for all $i \leqslant n$. After replacing $t$ by $t a_{n}$, if necessary, we may assume 
that $w(t)=1$. In this case $N=\operatorname{Ker}(h)$ is not a $\mathbb{Z}$-torsion module. Instead we find that

$$
R \otimes_{f} I_{\pi} \cong R /(a+1) \oplus(R /(2, a-1))^{n-1} \oplus M,
$$

where $M$ is an indecomposable $R$-module with underlying abelian group $\mathbb{Z} \oplus \mathbb{Z} / 2 \mathbb{Z}$ and $R$-action determined by $a \cdot(m,[n])=(m,[m+n])$ for all $(m,[n]) \in \mathbb{Z} \oplus \mathbb{Z} / 2 \mathbb{Z}$. In particular, the augmentation module $\mathbb{Z}$ is not a summand of $R \otimes_{f} I_{\pi}$. On the other hand, $R \otimes_{f} J_{\pi}$ does have $\mathbb{Z}$ as a summand. Therefore $R \otimes_{f} I_{\pi}$ and $R \otimes_{f} J_{\pi}$ are not stably isomorphic, and so $\left[I_{\pi}\right] \neq\left[J_{\pi}\right]$.

Thus $|G|$ is not divisible by 4 , so $|G|=2 m$ for some odd $m$.

In particular, if $w\left(G_{v}\right) \neq 1$ then $G_{v} \cong D_{2 m}$ for some odd $m$.

Theorem 7.4. Let $P$ be an indecomposable nonorientable $\mathrm{PD}_{3}$-complex with $\pi=\pi_{1}(P)$ virtually free. If $\pi$ has an orientation-reversing element of order 2 then $P \simeq R P^{2} \times S^{1}$.

Proof. Since $\pi$ is indecomposable and has nontrivial torsion, $\pi=\pi \mathcal{G}$, where $(\mathcal{G}, \Gamma)$ is a reduced finite graph of finite groups. At least one vertex group has an orientation-reversing element, by Theorem 7.1. If there is an edge $e$ such that $G_{o(e)}$ is orientable and $G_{t(e)}$ is nonorientable then $G_{e}$ must be cyclic of odd order, since $G_{t(e)} \cong \mathbb{Z} / m \mathbb{Z} \rtimes \mathbb{Z} / 2 \mathbb{Z}$ with $m$ odd, by Lemma 7.3. But then it is properly contained in each of its normalizers, contradicting the Centralizer Condition. Thus we may assume that all vertex groups are orientation-reversing. Hence they are all such semidirect products, and the edge groups are $\mathbb{Z} / 2 \mathbb{Z}$. Thus each vertex group has an unique conjugacy class of orientation-reversing elements of order 2 .

Suppose that there is a vertex group of order $2 m>2$. On passing to a subgroup of finite index, if necessary, we may assume that $\pi \cong F(r) \rtimes G$, where $G$ has order $2 p$, for some odd prime $p$. Then the vertex groups must all be isomorphic to $G$, and $G \cong D_{2 p}$, by the Normalizer and Centralizer Conditions.

Let $T$ be a maximal tree in $\Gamma$. Then $T$ omits at most one edge of $\Gamma$, since the centralizer of an orientation-reversing element of order 2 is finite or has two ends.

Suppose first that $\Gamma$ is a tree. Let $f: \mathbb{Z}[\pi] \rightarrow R=\mathbb{Z}[a] /\left(a^{2}-1\right)$ be the epimorphism induced by $w$. Then $R \otimes_{f} I(\pi) \cong R /(a+1) \oplus M$, where $M$ is a $\mathbb{Z}$-torsion module. Therefore $[I(\pi)] \neq[D I(\pi)]$, by Lemma 7.2, and so $\Gamma$ cannot be a tree.

If $\beta_{1}(\Gamma)=1$ then $\pi$ has a presentation

$$
\left\langle\begin{array}{l|l}
a, b_{1}, \ldots, b_{n}, t \mid \begin{array}{l}
b_{i}^{p}=a b_{i} a^{-1} b_{i}^{-\varepsilon}=a^{2}=1 \forall i \leqslant n \\
t a=a t
\end{array}
\end{array}\right\},
$$

where $\varepsilon=1$ if $G$ is cyclic and $\varepsilon=-1$ if $G$ is dihedral. Moreover, $w(a)=-1, w\left(b_{i}\right)=1$ for all $i \leqslant n$ and $w(t)=1$. Hence

$$
R \otimes_{f} I(\pi) R /(a+1) \oplus R /(a-1) \oplus(R /(p, a-\varepsilon))^{n},
$$

and so the $\mathbb{Z}$-torsion of $R \otimes_{f} I(\pi)$ is $(\mathbb{Z} / p \mathbb{Z})^{n}$, with $a$ acting as multiplication by $\varepsilon=-1$. On the other hand,

$$
R \otimes_{f} D I(\pi) \cong R /(a-1) \oplus R /(a+1) \oplus N^{n},
$$

where $N \cong R^{2} / R(p,-a-\varepsilon)$ is generated by two elements $n, n^{\prime}$, with $p n=(a+\varepsilon) n^{\prime}$. Let $v=(a-\varepsilon) n$. Then $v \neq 0$, but $p v=(a-\varepsilon)(a+\varepsilon) n^{\prime}=0$ and $(a+\varepsilon) v=(a+\varepsilon)(a-\varepsilon) n=0$. Thus $a$ acts as multiplication by $-\varepsilon$ on this nontrivial $p$-torsion element of $N$. Since $-\varepsilon \not \equiv \varepsilon \bmod p$ it follows that $R \otimes_{f} I(\pi)$ and $R \otimes_{f} D I(\pi)$ are not stably isomorphic, and so $[I(\pi)] \neq[D I(\pi)]$. 
Since $\pi$ is infinite, the only remaining possibility is that $\Gamma$ has one vertex $v$ and one edge $e$, with $G_{e}=G_{v}=\mathbb{Z} / 2 \mathbb{Z}$. Thus $\pi \cong \mathbb{Z} / 2 \mathbb{Z} \oplus \mathbb{Z}$, and so $P \simeq R P^{2} \times S^{1}$, by Theorem 5.8 .

\subsection{Indecomposable nonorientable 3-manifolds}

The main result of this chapter (Theorem 7.10) is relatively easy (and no doubt well-known) in the case of irreducible 3-manifolds, as we may use the Sphere Theorem, as strengthened by Epstein [36].

Theorem 7.5. Let $M$ be an indecomposable nonorientable 3-manifold with fundamental group $\pi$. If $\pi$ has infinitely many ends then $\pi \cong \pi^{+} \rtimes \mathbb{Z} / 2 \mathbb{Z}^{-}$and $\pi^{+}$is torsion-free, but not free.

Proof. Let $\mathcal{P}$ be a maximal set of pairwise non-parallel 2-sided projective planes in $M$. Then $\mathcal{P}$ is nonempty, since $M$ is indecomposable and $\pi$ has infinitely many ends. In particular, $\pi \cong \pi^{+} \rtimes \mathbb{Z} / 2 \mathbb{Z}^{-}$, since the inclusion of a member of $\mathcal{P}$ splits $w=w_{1}(M): \pi \rightarrow \mathbb{Z} / 2 \mathbb{Z}$. The preimage of $\mathcal{P}$ in $M^{+}$is a set $\mathcal{P}^{+}$of disjoint 2-spheres, and each of the components of $M^{+} \backslash \mathcal{P}^{+}$is a double cover of a component of $M \backslash \mathcal{P}$. Each such component of $M \backslash \mathcal{P}$ is indecomposable [36].

Suppose that $M \backslash \mathcal{P}$ has a component $Y$ such that $\pi_{1}(Y)$ is virtually free. The double $D Y$ is indecomposable (see Lemma 1.4), nonorientable and $\pi_{1}(D Y)$ is virtually free. Moreover, $D Y \cong R P^{2} \times S^{1}$, by Theorem 7.4, since the inclusion of a boundary component of $Y$ splits $w$. Therefore $Y \cong R P^{2} \times[0,1]$. This is contrary to the hypothesis that the members of $\mathcal{P}$ are non-parallel. Thus the components of $M \backslash \mathcal{P}$ are punctured aspherical 3-manifolds.

Let $\Gamma$ be the graph with vertex set $\pi_{0}(M \backslash \mathcal{P})$ and edge set $\mathcal{P}$, with an edge joining contiguous components. Then $\pi^{+} \cong G * F(s)$, where $G$ is a free product of $\mathrm{PD}_{3}$-groups (corresponding to the fundamental groups of the components of $M \backslash \mathcal{P}$ ), and $s=\beta_{1}(\Gamma)$. Hence $\pi^{+}$is torsion-free.

We remark also that each component $Y$ of $M \backslash \mathcal{P}$ has an even number of boundary components, since $\chi(\partial Y)$ is even (for any odd-dimensional manifold $Y$ ), by Poincaré duality. Thus the vertices of the graph $\Gamma$ have even valence.

Example. The canonical involution $\iota$ of the topological group $T^{3}=\mathbb{R}^{3} / \mathbb{Z}^{3}$ has 8 isolated fixed points (the points of order 2). Let $X$ be the complement of an equivariant open regular neighbourhood of the fixed point set, and let $M=D(X /\langle\iota\rangle)$. Then $M$ is indecomposable and nonorientable, and $\pi \cong$ $\left(\mathbb{Z}^{3} * \mathbb{Z}^{3} * F(7)\right) \rtimes \mathbb{Z} / 2 \mathbb{Z}^{-}$.

\subsection{Indecomposable nonorientable $\mathrm{PD}_{3}$-complexes}

We return to the setting of $\mathrm{PD}_{3}$-complexes.

Lemma 7.6. Let $P$ be an indecomposable nonorientable $\operatorname{PD}_{3}$-complex with $\pi=\pi_{1}(P)$ and $w=w_{1}(P)$. Suppose that $\pi \cong \pi \mathcal{G}$, where $(\mathcal{G}, \Gamma)$ is an admissible graph of groups.

(1) If $e$ is an edge with $G_{o(e)}$ or $G_{t(e)}$ infinite, then $G_{e}=\mathbb{Z} / 2 \mathbb{Z}^{-}$.

(2) If $P \nsucceq S^{2} \widetilde{\times} S^{1}$ then $\pi \cong \pi^{+} \rtimes \mathbb{Z} / 2 \mathbb{Z}^{-}$.

(3) If all finite vertex groups are 2-groups, then they are nonorientable, and all edge groups are $\mathbb{Z} / 2 \mathbb{Z}^{-}$. Proof. Suppose first that the vertex groups are all finite. Then $P \simeq S^{2} \widetilde{\times} S^{1}$ (if all the vertex groups are orientation-preserving) or $R P^{2} \times S^{1}$ (otherwise), by Theorems 7.1 and 7.4, respectively, and so the 
lemma holds. Hence we may assume that $(\mathcal{G}, \Gamma)$ has at least one infinite vertex group $G_{v}$ and at least one edge $e$ with $o(e)=v$ or $t(e)=v$. If $w(g)=1$ for some $g \in G_{e}$ of prime order then both $G_{o(e)}^{+}$and $G_{t(e)}^{+}$would be finite, by the Centralizer Condition. But then $G_{v}$ would be finite, contrary to hypothesis. Thus $G_{e}=\mathbb{Z} / 2 \mathbb{Z}^{-}$, and the inclusion of $G_{e}$ into $\pi$ splits $w$, so $\pi \cong \pi^{+} \rtimes \mathbb{Z} / 2 \mathbb{Z}^{-}$.

Suppose that all finite subgroups are 2-groups. Let $f$ be an edge such that the vertex groups $G_{o(f)}$ and $G_{t(f)}$ are finite. If $G_{f}=G_{o(f)}\left(\right.$ or $\left.G_{f}=G_{t(f)}\right)$ then $f$ must be a loop isomorphism, since $(\mathcal{G}, \Gamma)$ is reduced. But then $C_{\pi}\left(G_{f}\right)$ is infinite, and so $G_{f}=\mathbb{Z} / 2 \mathbb{Z}^{-}$, by the Centralizer Condition. Since $(\mathcal{G}, \Gamma)$ is reduced, $f$ must be the only edge, contrary to the assumption that there is an infinite vertex group. Thus we may assume that $G_{o(f)}$ and $G_{t(f)}$ each properly contain $G_{f}$. Since $G_{o(f)}$ and $G_{t(f)}$ are 2-groups and hence nilpotent, $N_{\pi}\left(G_{f}\right)$ is infinite, by the Normalizer Condition. Since $C_{\pi}\left(G_{f}\right)$ has finite index in $N_{\pi}\left(G_{e}\right)$ we must have $G_{f}=\mathbb{Z} / 2 \mathbb{Z}^{-}$, by the Centralizer Condition. Since $\Gamma$ is connected it follows easily that every finite vertex group is nonorientable and every edge group is $\mathbb{Z} / 2 \mathbb{Z}^{-}$.

The next two lemmas consider two parallel special cases, involving a prime $p$, which is odd or 2, respectively.

Lemma 7.7. Let $P$ be an indecomposable $\mathrm{PD}_{3}$-complex with $\pi=\pi_{1}(P) \cong \kappa \rtimes W$, where $\kappa$ is orientable and torsion-free, and $W$ has order $2 p$, for some odd prime $p$. Then $P$ is orientable.

Proof. Suppose that $P$ is not orientable. Then $\pi$ and $\kappa$ are infinite. Since $\pi$ has a subgroup $W$ of finite order $>2$, we may assume that $\pi \cong \pi \mathcal{G}$, where $(\mathcal{G}, \Gamma)$ is an admissible graph of groups with $r \geqslant 1$ finite vertex groups and at least one edge. Let $s=\beta_{1}(\Gamma)$.

Each finite vertex group is mapped injectively by any projection from $\pi$ onto $W$ with kernel $\kappa$. If a vertex group $G_{v}$ has prime order then every edge $e$ with one vertex at $v$ is a loop isomorphism, since $(\mathcal{G}, \Gamma)$ is reduced. But then $\Gamma$ has just one vertex and $\pi \cong G_{v} \rtimes F$, which contradicts the hypothesis. Hence all finite vertex groups are isomorphic to $W$. If an edge $e$ is a loop isomorphism then $G_{e}^{+} \cong \mathbb{Z} / p \mathbb{Z}$ has infinite normalizer, contradicting the Centralizer Condition. If there is an edge $e$ with $G_{e}$ of order $p$ then both of the vertex groups $G_{o(e)}$ and $G_{t(e)}$ are finite, by Lemma 7.6. But then $\left[G_{o(e)}: G_{e}\right]=\left[G_{t(e)}: G_{e}\right]=2$, and so $N_{\pi}\left(G_{e}\right)$ is infinite, which again contradicts the Centralizer Condition. Since the orientation character $w$ factors through $W$ it follows that every edge group is $\mathbb{Z} / 2 \mathbb{Z}^{-}$and $w$ is nontrivial on every vertex group.

Since each edge group is $\mathbb{Z} / 2 \mathbb{Z}^{-}, w$ is nontrivial on each vertex group and so $\pi^{+}=\pi \mathcal{G}^{+}$, where $\left(\mathcal{G}^{+}, \Gamma\right)$ is a graph of groups with the same underlying graph $\Gamma$, trivial edge groups and vertex groups $G_{v}^{+}$, for all $v \in V(\Gamma)$. Hence $\pi^{+} \cong G * F(s) * J$, where $G$ is a free product of orientable $\mathrm{PD}_{3}$-groups and $J$ is a free product of $r$ copies of $\mathbb{Z} / p \mathbb{Z}$. We have $J \cong F(t) \rtimes \mathbb{Z} / p \mathbb{Z}$ for some $t \geqslant 0$. (In fact, $t=(p-1)(r-1)$, by a simple virtual Euler characteristic argument.)

Let $a \in \pi$ be such that $a^{2}=1$ and $w(a)=-1$. The involution of $\pi^{+}$induced by conjugation by $a$ maps each indecomposable factor which is not infinite cyclic to a conjugate of an isomorphic factor [47]. However, its behaviour on the free factor $F(s)$ may be more complicated. The subgroup $\lambda \cong \kappa \rtimes \mathbb{Z} / 2 \mathbb{Z}^{-}$ generated by $\kappa$ and $a$ is also the group of a $\mathrm{PD}_{3}$-complex, since it has finite index in $\pi$.

Let $w: \mathbb{Z}[\pi] \rightarrow R=\mathbb{Z}[\langle a\rangle]=\mathbb{Z}[a] /\left(a^{2}-1\right)$ be the linear extension of the orientation character. Then $I(\langle a\rangle) \cong \widetilde{\mathbb{Z}}=R /(a+1)$. We may factor out the action of $\pi^{+}$on a $\mathbb{Z}[\pi]$-module by tensoring with $R$. The derived sequence of the functor $R \otimes_{w}$ - applied to the augmentation sequence

$$
0 \rightarrow I(\pi) \rightarrow \mathbb{Z}[\pi] \rightarrow \mathbb{Z}
$$


gives an exact sequence

$$
0 \rightarrow H_{1}(\pi ; R)=\kappa / \kappa^{\prime} \rightarrow R \otimes_{w} I(\pi) \rightarrow R \rightarrow \mathbb{Z} \rightarrow 0 .
$$

The inclusion of $\langle a\rangle$ into $\pi$ splits the epimorphism from $R \otimes_{w} I(\pi)$ onto $I(\langle a\rangle)$, and so $R \otimes_{w} I(\pi) \cong$ $\kappa / \kappa^{\prime} \oplus \widetilde{\mathbb{Z}}$.

Let $\gamma$ be the normal subgroup of $\pi$ generated by $G \cup F(s)$, and let $H$ be the image of $\gamma$ in $\kappa / \kappa^{\prime}$. Then similar arguments show that

$$
R \otimes_{w} I(\pi)=H \oplus\left(R \otimes_{w} I(\pi / \gamma)\right) \quad \text { and } \quad R \otimes_{w} I(\lambda)=H \oplus\left(R \otimes_{w} I(\lambda / \gamma)\right) .
$$

The group $J$ and its free normal subgroup $F(t)$ have presentations

$$
J=\left\langle b_{i}, 1 \leqslant i \leqslant r \mid b_{i}^{p}=1 \forall i\right\rangle \quad \text { and } \quad F(t)=\left\langle x_{i, j}, 1 \leqslant i \leqslant r-1,1 \leqslant j \leqslant p-1 \mid\right\rangle,
$$

where $x_{i, j}$ has image $b_{1}^{j} b_{i+1}^{-j}$ in $J$, for $1 \leqslant i \leqslant r-1$ and $1 \leqslant j \leqslant p-1$.

The quotient $\pi /\langle G\rangle\rangle$ is the fundamental group of the (possibly unreduced) graph of groups $(\overline{\mathcal{G}}, \Gamma$ ) with vertex groups $W$ (or $\mathbb{Z} / 2 \mathbb{Z}^{-}$) and edge groups $\mathbb{Z} / 2 \mathbb{Z}^{-}$, obtained by replacing each infinite vertex group $G_{v}$ of $(\mathcal{G}, \Gamma)$ by $G_{v} / G_{v}^{+}=\mathbb{Z} / 2 \mathbb{Z}^{-}$. Thus if $W$ is abelian (and so has an unique element of order 2) then $\pi /\langle G\rangle \cong(F(s) * J) \times \mathbb{Z} / 2 \mathbb{Z}^{-}$. Hence $\pi / \gamma \cong J \times \mathbb{Z} / 2 \mathbb{Z}^{-}$and $\lambda / \gamma \cong F(t) \times \mathbb{Z} / 2 \mathbb{Z}^{-}$, and so

$$
R \otimes_{w} I(\pi / \gamma) \cong(R /(p, a-1))^{r} \oplus \widetilde{\mathbb{Z}} \quad \text { and } \quad R \otimes_{w} I(\lambda / \gamma) \cong(R /(a-1))^{t} \oplus \widetilde{\mathbb{Z}}=\mathbb{Z}^{t} \oplus \widetilde{\mathbb{Z}} .
$$

The quotient ring $R / p R=\mathbb{F}_{p}[a] /\left(a^{2}-1\right)$ is semisimple, and so $p$-torsion $R$-modules have unique factorizations as sums of simple modules. Since $I(\pi) \otimes_{w} R$ and $I(\lambda) \otimes_{w} R$ satisfy Corollary 2.4.1 (and projective $R$-modules are $\mathbb{Z}$-torsion-free), the $p$-torsion submodule of $R \otimes_{w} I(\pi)$ has the same numbers of summands of types $R /(p, a-1)$ and $R /(p, a+1)$, and similarly for $R \otimes_{w} I(\lambda)$. Since $R \otimes_{w} I(\lambda / \gamma)$ is $p$-torsion-free, the number of summands of types $R /(p, a-1)$ and $R /(p, a+1)$ in $H$ must also be equal. On the other hand, $R \otimes_{w} I(\pi / \gamma)$ has $r>0$ summands of type $R /(p, a-1)$ and none of type $R /(p, a+1)$. These conditions are inconsistent, and so $\pi$ is not the group of a nonorientable $\mathrm{PD}_{3}$-complex.

If $W$ is not abelian then it has an unique conjugacy class of elements of order 2 , and $\pi / \gamma \cong J \rtimes \mathbb{Z} / 2 \mathbb{Z}^{-}$ and $\lambda / \gamma \cong F(t) \rtimes \mathbb{Z} / 2 \mathbb{Z}^{-}$have presentations

$$
\left\langle a, b_{i}, 1 \leqslant i \leqslant r \mid a^{2}=1, b_{i}^{p}=1, a b_{i} a=b_{i}^{-1} \forall i\right\rangle,
$$

and

$$
\left\langle a, x_{i, j}, 1 \leqslant i \leqslant r-1,1 \leqslant j \leqslant p-1 \mid a^{2}=1, a x_{i j} a=x_{i, p-j} \forall i, j\right\rangle,
$$

respectively. (In particular, $\lambda / \gamma \cong F(t / 2) * \mathbb{Z} / 2 \mathbb{Z}^{-}$.) In this case

$$
R \otimes_{w} I(\pi / \gamma) \cong(R /(p, a+1))^{r} \oplus \widetilde{\mathbb{Z}} \quad \text { and } \quad R \otimes_{w} I(\lambda / \gamma) \cong R^{t / 2} \oplus \widetilde{\mathbb{Z}} \text {. }
$$

Consideration of the $p$-torsion submodules again shows that the conclusion of Corollary 2.4.1 does not hold, and so $\pi$ is not the group of a nonorientable $\mathrm{PD}_{3}$-complex. Thus $P$ must be orientable.

The case $p=2$ involves slightly different calculations.

Lemma 7.8. Let $P$ be an indecomposable $\mathrm{PD}_{3}$-complex with $\pi=\pi_{1}(P) \cong \kappa \rtimes W$, where $\kappa$ is orientable and torsion-free, and $W$ has order 4 . Then $P$ is orientable. 
Proof. As in Lemma 7.7, we suppose that $P$ is not orientable, so $\pi$ and $\kappa$ are infinite, and may assume that $\pi \cong \pi \mathcal{G}$, where $(\mathcal{G}, \Gamma)$ is an admissible graph of groups with $r \geqslant 1$ finite vertex groups and at least one edge. We continue the notation $J, \gamma, a$ and $R$ from Lemma 7.7. Note that $J$ is now a free product of $r$ copies of $\mathbb{Z} / 2 \mathbb{Z}$.

The inclusions of the edge groups split $w$, by Lemma 7.6. In this case $W \cong(\mathbb{Z} / 2 \mathbb{Z})^{2}=\mathbb{Z} / 2 \mathbb{Z} \oplus \mathbb{Z} / 2 \mathbb{Z}^{-}$ and has two orientation-reversing elements. The quotient $\pi / \gamma$ is the group of a finite graph of groups with all vertex groups $W$ and edge groups $\mathbb{Z} / 2 \mathbb{Z}^{-}$. Since $J$ is a free product of cyclic groups, $\pi / \gamma$ has a presentation

$$
\left\langle a, b_{i}, 1 \leqslant i \leqslant r \mid a^{2}=1, b_{i}^{2}=\left(a w_{i}\right)^{2}=\left(a w_{i} b_{i}\right)^{2}=1 \quad \forall i\right\rangle,
$$

where $w_{1}=1$ and $w_{i} \in F(t)$ for $2 \leqslant i \leqslant r$. The free subgroup $F(t)$ has basis $\left\{x_{i} \mid 1 \leqslant i \leqslant r-1\right\}$, where $x_{i}$ has image $b_{1} b_{i+1}$ in $J$, and $\lambda / \gamma$ has a presentation

$$
\left\langle a, x_{i}, 1 \leqslant i \leqslant r-1 \mid a^{2}=1, a x_{i} a=x_{i} b_{i+1} w_{i+1} b_{i+1} w_{i+1}^{-1} \quad \forall i\right\rangle .
$$

In this case

$$
R \otimes_{w} I(\pi / \gamma) \cong(R /(2, a-1))^{r} \oplus \widetilde{\mathbb{Z}} \quad \text { and } \quad R \otimes_{w} I(\lambda / \gamma) \cong \mathbb{Z}^{r-1} \oplus \widetilde{\mathbb{Z}}
$$

Since $R /(2, a+1)=R /(2, a-1)$, torsion considerations do not appear to help. If $r>1$ we may instead compare the quotients by the $\mathbb{Z}$-torsion submodules, since finitely generated torsion-free $R$-modules are direct sums of copies of $R, \mathbb{Z}$ and $\widetilde{\mathbb{Z}}[\mathrm{CR}$, Theorem 74.3]. We again see that $\pi$ is not the group of a nonorientable $\mathrm{PD}_{3}$-complex.

The case when $p=2$ and $r=1$ requires a little more work. Let $N$ be the $R$-module presented by the transposed conjugate of $\left(\begin{array}{c}2 \\ a-1\end{array}\right)$. If $\{e, f\}$ is the standard basis for $R^{2}$ then $N=R^{2} / R(2 e+(a+1) f)$. The $\mathbb{Z}$-torsion submodule of $N$ is generated by the image of $(a-1) e$, and has order 2 , but is not a direct summand. The quotient of $N$ by its $\mathbb{Z}$-torsion submodule is generated by the images of $e$ and $f-e$, and is a direct sum $\mathbb{Z} \oplus \widetilde{\mathbb{Z}}$. In particular, it has no free summand. It now follows easily that $H \oplus \widetilde{\mathbb{Z}} \oplus R /(2, a-1)$ is not stably isomorphic to $H \oplus \widetilde{\mathbb{Z}} \oplus N$. Therefore the conclusion of Corollary 2.4.1 does not hold, and so $\pi$ is not the group of a nonorientable $\mathrm{PD}_{3}$-complex. Thus $P$ must be orientable.

Our final lemma is needed to cope with three exceptional cases.

Lemma 7.9. Let $G=H \rtimes \mathbb{Z} / 2 \mathbb{Z}$, where $H=T_{1}^{*}, O_{1}^{*}$ or $I^{*}$. Suppose that every element of $G$ of order divisible by 4 is in $H$. Then $G$ has a subgroup $W$ of order 6 such that $[W: W \cap H]=2$.

Proof. Let $g$ be an element of order 2 in $G \backslash H$.

Suppose first that $H=T_{1}^{*}$, with presentation

$$
\left\langle x, y, z \mid x^{2}=(x y)^{2}=y^{2}, z^{3}=1, z x z^{-1}=y, z y z^{-1}=x y\right\rangle .
$$

Then $\zeta T_{1}^{*}=\left\langle x^{2}\right\rangle$ has order 2 . The outer automorphism group $\operatorname{Out}\left(T_{1}^{*}\right)$ is generated by the class of the involution $\rho$ which sends $x, y$ and $z$ to $y^{-1}, x^{-1}$ and $z^{2}$, respectively. (See [Hi, page 221].) Hence $\rho$ preserves the subgroup $S$ of order 3 generated by $z$.

If conjugation by $g$ induces an inner automorphism of $T_{1}^{*}$ there is an $h \in T_{1}^{*}$ such that $g x g^{-1}=h x h^{-1}$ for all $x \in T_{1}^{*}$. Then $g h=h g$ and $h^{2}$ is central in $T_{1}^{*}$, so $\left(h^{-1} g\right)^{2}=h^{2}$ has order dividing 4 . Therefore $h^{-1} g$ has order 2 , by hypothesis. 
Otherwise we may assume that there is an $h \in G^{+}$such that $g x g^{-1}=h \rho(x) h^{-1}$ for all $x \in T_{1}^{*}$, and so $\rho$ is conjugation by $h^{-1} g$. Since $\rho$ is an involution $\left(h^{-1} g\right)^{2}$ is central in $T_{1}^{*}$. We again see that $h^{-1} g$ has order 2. In each case $h^{-1} g$ normalizes $S$, so $W=\left\langle S, h^{-1} g\right\rangle$ has order 6 , while $h^{-1} g \notin H$, so $[W: W \cap H]=2$.

The commutator subgroup of $O_{1}^{*}$ is $T_{1}^{*}$. Since this is a characteristic subgroup, it is preserved by $g$. The group $T_{1}^{*}$ is a non-normal subgroup of $I^{*}$, of index 5. Since $g$ acts as an involution on the set of conjugates of $T_{1}^{*}$ we may assume that it preserves $T_{1}^{*}$. In each case the lemma follows easily from its validity for $H=T_{1}^{*}$.

We may now give our main result.

Theorem 7.10. Let $P$ be an indecomposable nonorientable $\mathrm{PD}_{3}$-complex such that $\pi=\pi_{1}(P)$ has infinitely many ends. Then:

(1) $\pi \cong \pi \mathcal{G}$, where $(\mathcal{G}, \Gamma)$ is an admissible graph of groups with all vertex groups one-ended and all edge groups $\mathbb{Z} / 2 \mathbb{Z}^{-}$.

(2) $\pi \cong \pi^{+} \rtimes \mathbb{Z} / 2 \mathbb{Z}^{-}$.

(3) $\pi^{+} \cong G * H$, where $G$ is a nontrivial free product of $\mathrm{PD}_{3}$-groups and $H$ is free. In particular, $\pi^{+}$is torsion-free.

Proof. Let $\pi \cong \pi \mathcal{G}$, where $(\mathcal{G}, \Gamma)$ is an admissible graph of groups. At least one vertex group is infinite, for otherwise $\pi$ has two ends, by Theorems 7.1 and 7.4. Hence $\pi^{+} \cong G * H$, where $G$ is a nontrivial free product of $\mathrm{PD}_{3}$-groups and $H$ is virtually free. Therefore $\pi^{+}$is virtually torsion-free. Let $\kappa$ be the intersection of the conjugates in $\pi$ of a torsion-free subgroup of finite index in $\pi^{+}$, and let $\phi: \pi \rightarrow \pi / \kappa$ be the canonical projection. Then $\kappa$ is orientable, torsion-free and of finite index, and $w$ factors through $\pi / \kappa$.

If $F$ is a finite subgroup then $\left.\phi\right|_{F}$ is injective, and $\phi^{-1}(\phi(F))$ has finite index in $\pi$. Hence $\phi^{-1}(\phi(F))$ has a graph of groups structure in which all finite vertex groups are isomorphic to subgroups of $F$. In particular, if $F$ is a nonorientable 2-group then at least one of these vertex groups is a nonorientable 2-group, and so there is a $g \in F$ such that $g^{2}=1$ and $w(g)=-1$, by part (3) of Lemma 7.6. Hence if, moreover, $F$ is cyclic then it has order 2.

Assume that there is a nonorientable finite vertex group $G_{v}$. Then $G_{v}$ has a nonorientable Sylow 2-subgroup $S(2)$, and so there is a $g \in S(2)$ such that $g^{2}=1$ and $w(g)=-1$. The orientable subgroup $G_{v}^{+}$has periodic cohomology, with period dividing 4, by Theorem 6.8. Moreover, every element of $G_{v}$ divisible by 4 is in $G_{v}^{+}$, by the argument of the previous paragraph.

Let $g$ be an element of order 2 whose image generates $G_{v} / G_{v}^{+}$. We may assume that $G_{v}^{+} \cong B \times \mathbb{Z} / d \mathbb{Z}$, where $B$ is either $\mathbb{Z} / a \mathbb{Z} \rtimes Q\left(2^{i}\right)$ (with $a$ odd and $i \geqslant 3$ ), $T_{k}^{*}$ or $O_{k}^{*}$ (for some $k \geqslant 1$ ), $I^{*}, P_{48 \cdot 3^{k-1} a}^{\prime \prime}$ (with $a>1$ odd) or $A(a, e)$ (with $a>1$ odd and $e \geqslant 1$ ). Suppose first that $G_{v}^{+}$is not a 2-group. Then it has a nontrivial subgroup $S$ of order $p$, for some odd prime $p$. If $d>1$ we may assume that $p$ divides $d$, and then $S$ is characteristic in $G_{v}^{+}$. This is also the case if $G_{v}^{+}$is isomorphic to $\mathbb{Z} / a \mathbb{Z} \rtimes Q(8), A(a, e)$ or $P_{48 \cdot 3^{k-1} a}^{\prime \prime}$ with $a$ odd (so $p$ divides $a$ or $p=3$ ), or $T_{k}^{*}$ or $O_{k}^{*}$ with $k>1$ (so $p=3$ ). In these cases $S$ is normalized by $g$, and the subgroup $H$ generated by $S$ and $g$ has order $2 p$. The remaining possibilities are that $G_{v}^{+}$is isomorphic to $T_{1}^{*} \times \mathbb{Z} / d \mathbb{Z}, O_{1}^{*} \times \mathbb{Z} / d \mathbb{Z}$ or $I^{*} \times \mathbb{Z} / d \mathbb{Z}$. For these cases we appeal to Lemma 7.9 , to see that $G_{v}$ has a nonorientable subgroup $W$ of order $2 p$. 
Since $\phi^{-1} \phi(W)$ has finite index in $\pi$, it is again the group of a nonorientable $\mathrm{PD}_{3}$-complex. This complex has an indecomposable factor whose group has $W$ as one of its finite vertex groups, and so has fundamental group $\kappa \rtimes W$. But this factor is nonorientable, and so contradicts Lemma 7.7.

Therefore we may assume that $G_{v}^{+}$is a 2-group. If $S(2)^{+} \neq 1$ (i.e., if $G_{v}^{+}$is a nontrivial 2-group) it is cyclic or generalized quaternionic, and so has an unique central element of order 2, by Lemma 5.3. Hence $G_{v}$ has a subgroup $W \cong \mathbb{Z} / 2 \mathbb{Z} \times \mathbb{Z} / 2 \mathbb{Z}^{-}$of finite index. Passage to $\phi^{-1} \phi(W)$ again leads to a contradiction, by Lemma 7.8 .

Therefore all finite vertex groups are orientable. But the graph $\Gamma$ is connected, and any edge connecting a finite vertex group to an infinite vertex group must be nonorientable, as in Lemma 7.6. Since at least one vertex group is infinite, there are no finite vertex groups.

The second assertion follows from part (2) of Lemma 7.6, and $\pi^{+}=\pi \mathcal{G}^{+}$is the fundamental group of a graph of groups $\left(\mathcal{G}^{+}, \Gamma\right)$ with the same underlying graph $\Gamma$, trivial edge groups and vertex groups $G_{v}^{+}$all $\mathrm{PD}_{3}$-groups. Hence $\pi^{+}$is torsion-free, but not free.

As observed at the end of $\S 2$, when $M$ is a 3-manifold and $(\mathcal{G}, \Gamma)$ is an admissible graph of groups such that $\pi_{1}(M)=\pi \mathcal{G}$, all vertices of $\Gamma$ have even valence. Can this observation be extended to the case of $\mathrm{PD}_{3}$-complexes? Although there are indecomposable orientable $\mathrm{PD}_{3}$-complexes which are not homotopy equivalent to 3-manifolds, it remains possible that every indecomposable nonorientable $\mathrm{PD}_{3}$-complex is homotopy equivalent to a 3-manifold.

We shall say that a nonorientable $\mathrm{PD}_{3}$-complex $P$ is algebraically $P^{2}$-irreducible if $\pi$ is indecomposable and $w_{1}(P)$ does not split.

Corollary 7.10.1. Let $P$ be a nonorientable $\mathrm{PD}_{3}$-complex. If $P$ is algebraically $P^{2}$-irreducible then either $P$ is aspherical or $P \simeq S^{2} \tilde{\times} S^{1}$.

Proof. This follows from Theorems 7.1, 7.4 and 7.10.

We may now refine the Centralizer Condition of Chapter 4.

Corollary 7.10.2. Let $P$ be a $\mathrm{PD}_{3}$-complex and $g \in \pi=\pi_{1}(P)$ a nontrivial element of finite order. If $C_{\pi}(g)$ is infinite then $g$ has order 2 and is orientation-reversing, and $C_{\pi}(g)=\langle g\rangle \times \mathbb{Z}$.

Proof. If $\pi$ is virtually free this follows from Theorem 7.4. Otherwise it follows from the Centralizer Condition and Theorem 7.10. 



\section{CHAPTER 8}

\section{Asphericity and 3-manifolds}

As a consequence of the results of Chapters 2-7, the study of $\mathrm{PD}_{3}$-complexes reduces largely to the study of $\mathrm{PD}_{3}$-groups. Every homotopy equivalence between aspherical 3-manifolds is homotopic to a homeomorphism. It is natural to ask also whether every $\mathrm{PD}_{3}$-group is a 3-manifold group. The fundamental groups of aspherical 3-manifolds which are Seifert fibred or are finitely covered by surface bundles may be characterized among all $\mathrm{PD}_{3}$-groups in simple group-theoretic terms. An affirmative answer in general would suggest that a large part of the study of 3-manifolds may be reduced to algebra.

We begin with some brief observations on the good behaviour of 3-manifold groups and the bad behaviour of $\mathrm{PD}_{n}$-groups when $n>3$. We then propose three possible approaches to showing that $\mathrm{PD}_{3}$ groups are 3-manifold groups. In $\S 3$ we give several lemmas relating to the third of these approaches, and in $\$ 4$ we state a number of results that we shall use in later chapters. (These results are not specifically about 3-dimensional issues, so we choose to treat them as "black box" tools.) In the final two sections we show that the groups of aspherical mapping tori, aspherical Seifert fibred 3-manifolds, and $\mathrm{Sol}^{3}$ manifolds are (virtually) the $\mathrm{PD}_{3}$-groups with nontrivial $\mathrm{FP}_{2}$ normal subgroups of infinite index. (We give two characterizations of groups of Seifert type, one using relatively simple arguments and an additional hypothesis, and the other using the much deeper Bowditch Theorem.) In Chapter 9 we shall see that allowing the subgroup to be merely subnormal, or even ascendant, leads to similar outcomes.

\subsection{Some properties of 3-manifold groups}

We shall henceforth use "3-manifold group" to mean the fundamental group of an aspherical closed 3-manifold, and shall summarize here a few of their properties. See [AFW] for further details.

If $M$ is a closed 3-manifold we may assume it has one 0-cell and one 3-cell, and equal numbers of 1and 2-cells. Hence $\pi=\pi_{1}(M)$ has a finite presentation of deficiency 0 ; if $M$ is aspherical this is clearly best possible, since $\beta_{1}\left(\pi ; \mathbb{F}_{2}\right)=\beta_{2}\left(\pi ; \mathbb{F}_{2}\right)$. Moreover, $\pi$ is FF, i.e., the augmentation module $\mathbb{Z}$ has a finite free $\mathbb{Z}[\pi]$-resolution.

As a consequence of the Geometrization Theorem of Perelman and Thurston, and the earlier work of Farrell and Jones, $\widetilde{K}_{0}(\mathbb{Z}[\pi])=\mathrm{Wh}(\pi)=0$ and the Novikov Conjecture holds for 3-manifold groups [77; 121]. Moreover, $\widetilde{M} \cong \mathbb{R}^{3}$, so $\pi$ is 1 -connected at $\infty$, and $\pi$ is residually finite. With the exception of certain proper graph manifolds, most aspherical 3-manifolds are virtually mapping tori [1].

The central role played by incompressible surfaces in the topology of 3-manifolds suggests strongly the importance of studying subgroups of infinite index in $\mathrm{PD}_{3}$-groups. There are substantial constraints on the subgroups of 3-manifold groups. Every finitely generated subgroup of a 3-manifold group is the fundamental group of a compact 3-manifold (possibly with boundary), by Scott's Core Theorem [123], and thus is finitely presentable. The Tits alternative holds; subgroups are either virtually solvable 
(in which case they are polycyclic) or contain nonabelian free groups. The centralizer of any element in a 3-manifold group is finitely generated. One of our goals is to try to establish such properties for $\mathrm{PD}_{3}$-groups.

In contrast, the product of two nonabelian $\mathrm{PD}_{2}^{+}$-groups contains a copy of $F(2) \times F(2)$, and so is a $\mathrm{PD}_{4}^{+}$-group which is not almost coherent. Moreover, any group of finite geometric dimension 2 is the fundamental group of a compact aspherical 4-manifold with boundary, obtained by attaching 1- and 2handles to $D^{4}$. On applying the reflection group trick of Davis [24] to the boundary we see that each such group embeds in a $\mathrm{PD}_{4}$-group. This leads to many interesting examples. For instance, there are $\mathrm{PD}_{4}$ groups which have Baumslag-Solitar groups as subgroups. Such groups have abelian subgroups which are not finitely generated, and are not residually finite (since this property is inherited by subgroups). Davis has shown that if $n \geqslant 4$ then there are $\mathrm{PD}_{n}$-groups which are not finitely presentable, and Leary has extended this to show that there are uncountably many such $\mathrm{PD}_{n}$-groups [102, Theorem 18.1].

Thus the position of $\mathrm{PD}_{3}$-groups is critical, being between the now fairly well delineated world of surfaces and 3-manifolds and the wilderness of exotic phenomena in dimensions $\geqslant 4$.

\subsection{Are $\mathrm{PD}_{3}$-groups 3-manifold groups?}

The key approaches to this question seem to be through

(1) splitting over proper subgroups/geometric group theory;

(2) homological algebra; or

(3) topology.

Of course, there are overlaps between these. The fact that $\mathrm{PD}_{2}$-groups are surface groups is one common ingredient.

We shall see that it is no loss of generality to assume that $G$ is orientable. It may also be convenient to assume also that $G$ is coherent, and, in particular, finitely presentable.

(1) This approach has been most studied, particularly in the form of the Cannon Conjecture: that an atoroidal Gromov hyperbolic $\mathrm{PD}_{3}$-group should be a cocompact lattice in $\operatorname{PSL}(2, \mathbb{C})$. There is a good exposition based on JSJ decompositions of (finitely presentable) $\mathrm{PD}_{3}$-groups and pairs of groups (as in [27]) in [152]. If one takes this approach it is natural to consider also the question of realizing $\mathrm{PD}_{3}$-pairs of groups.

Splitting of $\mathrm{PD}_{3}$-groups over proper subgroups was first considered by Thomas [142]. Kropholler showed that $\mathrm{PD}_{n}$-groups with max-c have canonical splittings along codimension-1 polycyclic subgroups [94]. (When $n=3$ such subgroups are $\mathbb{Z}^{2}$ or Kb.) Castel used [127] to give a JSJ decomposition for arbitrary $\mathrm{PD}_{3}$-groups (i.e., not assuming finite presentability), and showed that all $\mathrm{PD}_{3}$-groups have max-c [20]. (We give another proof of max-c for $\mathrm{PD}_{3}$-groups in Chapter 9, and state Kropholler's decomposition theorem in Chapter 10.)

In the simplest cases, $G$ is either solvable, of Seifert type or atoroidal. The solvable case is easy, and the Seifert case was settled by Bowditch [15]. (See Theorem 8.9.) Suppose that $G$ is atoroidal. If, moreover, $G$ acts geometrically on a locally compact CAT(0) space then it is Gromov hyperbolic [82]. Hence it is finitely presentable, $\widetilde{K}_{0}(\mathbb{Z}[G])=0$ and $\mathrm{Wh}(G)=0$, and so $K(G, 1)$ is a finite simple $\mathrm{PD}_{3}$-complex. Moreover $G$ has boundary $S^{2}$ [7]. (Note also the related result of [143] for the bounded 
case.) If the problem is stabilized by taking products with a closed manifold then surgery methods are applicable. In [39] it is shown that $G \times \mathbb{Z}^{d}$ is the fundamental group of an aspherical $(d+3)$-manifold, for all $d \geqslant 2$.

(2) The homological approach perhaps has the least prospect of success, as it starts from the bare definition of a $\mathrm{PD}_{3}$-group, and needs something else to connect with topology. However, it has proven useful in the subsidiary task of finding purely algebraic proofs for algebraic properties of 3-manifold groups, an activity that shall be pursued in later chapters. One can also show that if $G$ has sufficiently nice subgroups then it is a 3-manifold group. For instance, if $G$ has a nontrivial $\mathrm{FP}_{2}$ ascendant subgroup of infinite index, then either $G$ is the group of a Seifert fibred 3-manifold or it is virtually the group of a mapping torus. (See Chapters 9 and 10.)

This strategy seems to work best when there are subgroups which are surface groups. One relatively new ingredient is the Algebraic Core Theorem, which ensures that this is so if $G$ has an $\mathrm{FP}_{2}$ subgroup with one end. However, it remains possible that there may be $\mathrm{PD}_{3}$-groups which are simple groups, or even Tarski monsters, whose only proper subgroups are infinite cyclic. It is then unclear what to do. (The Davis construction may be used to give $\mathrm{PD}_{n}$-groups containing Tarski monsters, for all $n>3$ [122].)

(3) To the best of my knowledge, no-one has explored the third option in any detail. It has the advantage of direction connection with topology, but needs $G$ to be finitely presentable. Here one starts from the fact that if $P$ is an orientable $\mathrm{PD}_{3}$-complex then there is a degree-1 map $f: M \rightarrow P$ with domain a closed orientable 3-manifold. (This follows from the Atiyah-Hirzebruch Spectral Sequence, but can also be seen by "bare hands"; we may represent a generator of $H_{3}(P ; \mathbb{Z})$ by a finite union of tetrahedra, with faces identified in pairs. The resulting "Euler complex" has finitely many non-manifold points, which are cones over surfaces. Replacing these conical neighbourhoods by handlebodies gives a 3-manifold M.) Then $f$ is a homotopy equivalence if and only if $\operatorname{Ker}\left(f_{*}\right)=1$. Since $\pi_{1}(f)$ is surjective and $\pi_{1}(M)$ and $\pi=\pi_{1}(P)$ are finitely presentable, $\operatorname{Ker}\left(\pi_{1}(f)\right)$ is normally generated by finitely many elements of $\pi_{1}(M)$, which may be represented by the components of a link $L \subset M$. (The link $L$ is far from being unique!) We might hope to modify $M$ by Dehn surgery on $L$ to render the kernel trivial. This is possible if $P$ is homotopy equivalent to a closed orientable 3-manifold $N$, for $M$ and $N$ may then be obtained from each other by Dehn surgeries on links whose components are null-homotopic in $N$ [46]. However, there are $\mathrm{PD}_{3}$-complexes which are not homotopy equivalent to manifolds, and so this cannot be carried through in all cases. The known counterexamples have finite covering spaces which are homotopy equivalent to $\sharp^{r}\left(S^{2} \times S^{1}\right)$ for some $r \geqslant 0$. Thus it remains possible that every $\mathrm{PD}_{3}$-complex is virtually a 3-manifold, i.e., has a finite covering space which is homotopy equivalent to a closed orientable 3-manifold. If this is true it must be possible to $\operatorname{kill} \operatorname{Ker}\left(f_{*}\right)$ by surgery and passing to finite covering spaces.

In general, we might expect to encounter obstructions in $L_{3}(\pi, w)$ to obtaining a $\mathbb{Z}[\pi]$-homology equivalence by integral surgery. For instance, there are finite $\mathrm{PD}_{3}$-complexes with $\pi$ dihedral, but such groups do not act freely on homology 3-spheres. However, the validity of the Novikov conjecture for aspherical 3-manifolds suggests that such obstructions may never arise in the cases of most interest to us. (See $[77 ; 86]$.) In any case, we allow Dehn surgeries also.

We shall say that a link $L=\coprod_{i \leqslant m} L_{i}$ in a 3-manifold $N$ with an open regular neighbourhood $n(L)=$ $\bigsqcup_{i \leqslant m} n\left(L_{i}\right)$ admits a drastic surgery if there is a family of slopes $\gamma_{i} \subset \partial n\left(L_{i}\right)$ such that the normal 
closure of $\left\{\left[\gamma_{1}\right], \ldots,\left[\gamma_{n}\right]\right\}$ in $\pi_{1}(N \backslash n(L))$ meets the image of each peripheral subgroup $\pi_{1}\left(\partial n\left(L_{i}\right)\right)$ in a subgroup of finite index.

If $P$ is an aspherical orientable $\operatorname{PD}_{3}$-complex and $f: M \rightarrow P$ is a degree-1 map such that $\operatorname{Ker}\left(f_{*}\right)$ is represented by a link $L$ which admits a drastic surgery, then after the surgery we may assume that $\operatorname{Ker}\left(f_{*}\right)$ is normally generated by finitely many elements of finite order. Let $M=\sharp_{i=1}^{r} M_{i}$ be the decomposition into irreducibles. Since $P$ is aspherical, the map $f$ extends to a map $f_{\vee}: \bigvee_{i=1}^{r} M_{i} \rightarrow P$. Elementary considerations then show that $f_{\vee}$ restricts to a homotopy equivalence from one of the aspherical summands of $M$ to $P$.

Unfortunately, there are knots which admit no drastic surgery. The following example was suggested by Cameron Gordon. Let $M$ be an orientable 3-manifold which is Seifert fibred over the 2-orbifold $S^{2}(p, q, r)$, where $\frac{1}{p}+\frac{1}{q}+\frac{1}{r} \leqslant 1$, and let $K \subset M$ be a regular fibre. Let $\phi, \mu \subset \partial n(K)$ be a regular fibre and a meridian, respectively. Then surgery on the slope $s \mu+t \phi$ gives a 3-manifold which is Seifert fibred over $S^{2}(p, q, r, s)$, if $s \neq 0$, or is a connected sum of lens spaces, if $s=0$. If $s \neq 0$ the image of $\phi$ has infinite order in $\pi_{1}(N)$; otherwise, the image of $\mu$ has infinite order there. Thus no surgery on a regular fibre of $M$ is drastic. (We may modify this example to obtain one with $M$ not Seifert fibred, by replacing a tubular neighbourhood of another regular fibre by the exterior of a hyperbolic knot.)

However, we do have considerable latitude in our choice of link $L$ representing $\operatorname{Ker}\left(f_{*}\right)$. In particular, we may modify $L$ by a link homotopy, and so the key question may be whether every knot $K \subset M$ is homotopic to one which admits a drastic surgery.

The existence of $\mathrm{PD}_{3}$-complexes which are not homotopy equivalent to 3-manifolds shows that we cannot expect a stronger result, in which "contains the image of each peripheral subgroup $\pi_{1}\left(\partial \overline{n\left(L_{i}\right)}\right)$ " replaces "meets the image ... finite index" in the definition of drastic surgery. Can we combine Dehn surgery with passage to finite covers and varying $L$ by link-homotopy?

The argument for the existence of a degree-1 map $f: M \rightarrow P$ does not require us to assume a priori that $P$ be finite, nor even that $\pi_{1}(P)$ be finitely presentable. The latter condition is needed to ensure that $\operatorname{Ker}\left(f_{*}\right)$ is represented by a link in $M$. This leaves the question: are $\mathrm{PD}_{3}$-groups finitely presentable? Our strategy does not address this issue.

\subsection{Some reductions}

Lemma 8.1. Let $P=P_{1} \sharp P_{2}$ be a $\mathrm{PD}_{3}$-complex which is the connected sum of $\mathrm{PD}_{3}$-complexes which are virtually 3-manifolds. Then $P$ is virtually a 3-manifold.

Proof. Let $\widehat{P}_{i}$ be a finite regular covering space of $P_{i}$ which is homotopy equivalent to a closed 3manifold $M_{i}$, where $i=1,2$. Let $G_{i}=\operatorname{Aut}\left(\widehat{P}_{i} / P_{i}\right)$ and let $H$ be the kernel of the natural projection of $\pi_{1}(P)=\pi_{1}\left(P_{1}\right) * \pi_{1}\left(P_{2}\right)$ onto $G_{1} \times G_{2}$. Then the associated covering space $P_{H}$ with fundamental group $H$ is homotopy equivalent to a connected sum of copies of $M_{1}$ and $M_{2}$.

We shall assume henceforth that $P$ is aspherical.

Lemma 8.2. If an aspherical $\mathrm{PD}_{3}$-complex $P$ is virtually a 3-manifold then $P$ is homotopy equivalent to a 3-manifold.

Proof. Let $f: M \rightarrow \widehat{P}$ be a homotopy equivalence from a closed 3-manifold $M$ to a finite regular covering space $\widehat{P}$. Then $M$ is aspherical, and is either Seifert fibred, hyperbolic or Haken, by the Geometrization 
Theorem of Thurston and Perelman. If $M$ is Seifert fibred then $P$ is homotopy equivalent to a Seifert fibred 3-manifold [15, Corollary 15.2]. If $M$ is hyperbolic then $G=\operatorname{Aut}(\widehat{P} / P)$ is isomorphic to a group of isometries of $M$, by Mostow rigidity. The group $\Gamma$ of all lifts of such isometries to $\widetilde{M}=\mathbb{H}^{3}$ is isomorphic to $\pi_{1}(P)$ and acts properly discontinuously on $\mathbb{H}^{3}$. Since $\Gamma$ is torsion-free the action is free, and so $\mathbb{H}^{3} / \Gamma$ is a closed hyperbolic 3-manifold homotopy equivalent to $P$. If $M$ is Haken then it has a canonical JSJ decomposition into Seifert fibred and hyperbolic pieces, and a similar conclusion holds [158]. (Zimmermann assumes $M$ orientable, but his argument holds more generally.)

Thus we may further assume that $P$ is orientable. There is a degree-1 map $f: M \rightarrow P$ with domain a closed 3-manifold, as observed above. As every closed orientable 3-manifold is the target of a degree-1 map from a hyperbolic 3-manifold [83], we could also assume that $M$ is aspherical. As we may lose asphericity of $M$ under surgery, we shall settle for a simpler result.

Lemma 8.3. Let $P$ be an aspherical $\mathrm{PD}_{3}$-complex and $f: M \rightarrow P$ a degree-1 map. Then we may assume that the irreducible factors of $M$ are aspherical.

Proof. Let $M=\sharp_{i=1}^{i=k} M_{i}$ be a factorization of $M$ as a connected sum of irreducible 3-manifolds, with $M_{i}$ aspherical if $i \leqslant r$ and $\pi_{1}\left(M_{i}\right)$ finite, $\mathbb{Z}$ or $\mathbb{Z} / 2 \mathbb{Z} \oplus \mathbb{Z}$ if $i>r$. Since $P$ is aspherical, $f$ extends to a map $F: \bigvee_{i=1}^{i=k} M_{i} \rightarrow P$. If $\pi_{1}\left(M_{i}\right)$ is finite then $\left.F\right|_{M_{i}}$ is null-homotopic, while if $\pi_{1}\left(M_{i}\right)$ is isomorphic to $\mathbb{Z}$ or $\mathbb{Z} / 2 \mathbb{Z} \oplus \mathbb{Z}$ then $\left.F\right|_{M_{i}}$ factors through $S^{1}$. In either case the restriction to such terms has degree 0 . Hence $F$ induces a degree-1 map from $\sharp_{i=1}^{i=r} M_{i}$ to $P$.

Let $L \subset M$ be a link whose components represent a subset of $\pi_{1}(M)$ whose normal closure is $\operatorname{Ker}\left(f_{*}\right)$. We may assume that the number of components of $L$ is minimal among all such pairs $(f, L)$.

Lemma 8.4. If $P$ is an aspherical $\mathrm{PD}_{3}$-complex and $L$ admits a drastic surgery, then $P$ is homotopy equivalent to a 3-manifold.

Proof. After a drastic surgery on $L$ we may assume that $\operatorname{Ker}\left(f_{*}\right)$ is normally generated by finitely many elements of finite order. Let $M=N \sharp N^{\prime}$, where $\pi_{1}(N)$ is torsion-free and the fundamental groups of the irreducible summands of $N^{\prime}$ are finite. As in the previous lemma, $f$ factors through the collapse of $M$ onto $N$, and so induces a degree-1 map $g: N \rightarrow P$. This map is clearly $\pi_{1}$-injective, and so it is a homotopy equivalence.

\subsection{A compendium of frequently cited theorems}

Here we collate some useful general results about $\mathrm{PD}_{n}$-groups and related issues, in some cases simplified to meet our needs.

We begin with several results from [Bi]. We shall refer to these in the standard way, rather than give them names, but it may be useful to have the statements immediately at hand. (There are many other results in this book which we use, but shall not single out here.)

Theorem [Bi, Theorem 8.4]. Let $G$ be a finitely generated group such that $\mathrm{cd} G=2$ and let $N$ be a finitely generated, free normal subgroup of $G$. Then $G / N$ is virtually free.

(The original version of this theorem considers finitely generated groups $G$ such that cd $G=n<\infty$, and normal subgroups $N$ which are duality groups of dimension $n-1$; the conclusion is the same.) 
Lemma [Bi, Corollary 8.6]. Let $G$ be a group such that $\mathrm{cd} G=2$, and let $N$ be a normal $\mathrm{FP}_{2}$ subgroup of $G$. Then either $[G: N]$ is finite or $N$ is free.

Theorem [Bi, Theorem 8.8]. Let $G$ be a nonabelian group such that $\mathrm{cd} G=n<\infty$. Then:

(1) $\operatorname{cd} \zeta G<n$.

(2) If $\zeta G \cong \mathbb{Z}^{n-1}$ then $G^{\prime}$ is free.

It is easy to see that a torsion-free virtually polycyclic group of Hirsch length $n$ is a $\mathrm{PD}_{n}$-group. Conversely:

Theorem [Bi, Theorem 9.23]. Every virtually solvable $\mathrm{PD}_{n}$-group is virtually polycyclic.

The next result is particularly useful when $G$ is almost coherent, for then the base and associated subgroups are again $\mathrm{FP}_{2}$.

Theorem (Bieri-Strebel Theorem [10]). Let $G$ be an $\mathrm{FP}_{2}$ group with $G / G^{\prime}$ infinite. Then $G$ is an HNN extension with finitely generated base and associated subgroups.

Infinite covering spaces of closed $n$-manifolds are open, and so have cohomological dimension $<n$. In the PL case this is easy to see, as open PL $n$-manifolds collapse into their $(n-1)$-skeleton. Strebel showed that a similar result holds for subgroups of infinite index in $\mathrm{PD}_{n}$ groups [134].

Theorem (Strebel Theorem). Let $G$ be $a \mathrm{PD}_{n}$-group and let $H$ be a subgroup of infinite index in $G$. Then $\operatorname{cd} H<n$.

When $n=3$, either $H$ is free or cd $H=2$. Under more stringent conditions on the subgroup, Shapiro's Lemma with Poincaré duality (for each of the ambient and quotient groups) together imply the next result. (See [Hi, Theorem 1.19] for the general case.)

Theorem (PD Extension Theorem). Let $\pi$ be a $\mathrm{PD}_{n}$-group with a normal subgroup $K$ such that $\pi / K$ is $a \mathrm{PD}_{r}$-group. Then $K$ is a $\mathrm{PD}_{n-r}$-group if and only if it is $\mathrm{FP}_{[n / 2]}$.

The easiest part of the argument gives a useful special case of the Strebel Theorem. Let $A$ be a left $\mathbb{Z}[K]$-module, and let $W=\operatorname{Hom}_{\mathbb{Z}[K]}(\mathbb{Z}[\pi], A)$ be the coinduced module. Then $H^{s}(K ; A) \cong H^{s}(\pi ; W) \cong$ $H_{n-s}(\pi ; \bar{W})$, by Shapiro's Lemma and Poincaré duality for $\pi$. In particular, if $\pi / K$ is infinite then $H^{n}(K ; A)=0$ for all $A$, and so cd $K<n$.

Theorem 3.3 of [49] gives a criterion for the vanishing of certain cohomology modules for a group which is a union of an increasing chain of FP subgroups. In our context, their argument gives the following result, which we use together with the Strebel Theorem.

Theorem (Gildenhuys-Strebel Theorem). Let $G=\bigcup_{n \in I} G_{n}$ be the union of an increasing sequence of $\mathrm{FP}_{2}$, one-ended subgroups, indexed by an interval $I \subseteq \mathbb{N}$. If $\left[G_{n+1}: G_{n}\right]<\infty$ for all $n \in I$ and $\mathrm{cd} G \leqslant 2$, then the sequence is finite, so $G$ is $\mathrm{FP}, \operatorname{cd} G=2$ and $G$ has one end.

The enunciation of this theorem in [49] assumes that $G_{n}$ is FP, cd $G_{n}=r$ and $H^{s}\left(G_{n} ; \mathbb{Z}\left[G_{n}\right]\right)=0$ for all $s<r$ and all $n \geqslant 1$, and concludes that if $G$ is not finitely generated then $H^{s}(G ; W)=0$ for all free $\mathbb{Z}[G]$-modules $W$ and all $s \leqslant r$. However, the argument establishes the above assertion.

Kapovich and Kleiner have introduced ideas from "coarse geometry" to the study of $\mathrm{PD}_{n}$-groups [81]. Their principal results are formulated in terms of discrete groups acting on "coarse $\operatorname{PD}(n)$-complexes", 
and apply to $\mathrm{PD}_{n}$-groups through their Example 11.6. For our purposes, their main result is their Corollary 1.2:

Theorem (Algebraic Core Theorem). Let $G$ be an $\mathrm{FP}_{2}$ subgroup of infinite index in a $\mathrm{PD}_{3}$-group $\pi$. Then each factor of $G$ with one end is the ambient group of a $\mathrm{PD}_{3}$-pair. Hence either $G$ contains a surface group or $G$ is free. If $G$ has one end then $\chi(G) \leqslant 0$.

Finally, we note that if $(G, \Omega)$ is a $\mathrm{PD}_{n}$-pair of groups with nonempty boundary, we may double $G$ along its boundary to obtain a $\mathrm{PD}_{n}$-group $D(G, \Omega)$ in which $G$ embeds, and so questions about subgroups of $\mathrm{PD}_{n}$-pairs of groups may often be reduced to questions about subgroups of infinite index in $\mathrm{PD}_{n}$-groups. This is a special case of the following result [8, Theorem 8.4].

Theorem (Bieri-Eckmann Splitting Theorem). Let $(\mathcal{G}, \Gamma)$ be a finite graph of groups, in which all edge groups are $\mathrm{PD}_{n-1}$-groups. For each vertex $v$ let $\mathcal{E}_{v}=\sqcup_{o(e)=v} G_{v} / G_{e} \sqcup \sqcup_{t(e)=v} G_{v} / G_{e}$, and suppose that $\Omega_{v}$ is a $G_{v}$-set with finitely many $G_{v}$-orbits and all point stabilizers $\mathrm{PD}_{n-1}$-groups. Let $\Omega=\sqcup_{v \in V}\left(G \times_{G_{v}} \Omega_{v}\right)$ be the disjoint union of the induced $G$-sets. Then $(\pi \mathcal{G}, \mathcal{T})$ is a $\mathrm{PD}_{n}$-pair of groups if and only if $\left(G_{v}, \mathcal{E}_{v} \sqcup \Omega_{v}\right)$ is a $\mathrm{PD}_{n}$-pair of groups for all $v$.

We note here one immediate consequence of the Algebraic Core Theorem. If $(H, \mathcal{T})$ is a $\mathrm{PD}_{3}$-pair of groups then $\sum_{H \cdot \tau} \chi\left(H_{\tau}\right)=2 \chi(H)$ (where the sum is over representatives of the boundary components), by Lemma 3.1. If $r, s>1$ then $H=F(r) \times F(s)$ is finitely presentable and has one end. However, $\chi(H)=(1-r)(1-s)>0$, and so no such product is a subgroup of a $\mathrm{PD}_{3}$-group. (This was first proven in [101].) It is well known that $F(r) \times F(s)$ has subgroups which are finitely generated but not $\mathrm{FP}_{2}$. Thus this result supports the hope that $\mathrm{PD}_{3}$-groups may all be coherent.

We shall also use the following simple consequence of the Strebel Theorem. If $f: G \rightarrow H$ is a homomorphism of $\mathrm{PD}_{n}^{+}$-groups, then $\operatorname{deg} f$ is the integer $g \geqslant 0$ such that $f_{*}[G]= \pm d[H]$, for any choice of fundamental classes $[G],[H]$.

Lemma 8.5. Let $G$ and $H$ be $\mathrm{PD}_{n}^{+}$-groups and $\phi: G \rightarrow H$ a homomorphism.

(1) If $\operatorname{deg} \phi \neq 0$ then $[H: \phi(G)]$ is finite and $\operatorname{cd} \operatorname{Ker}(\phi)<n$.

(2) If $\phi: G \rightarrow H$ is a monomorphism then $[H: \phi(G)]$ is finite and $|\operatorname{deg} \phi|=[H: \phi(G)]$.

Proof. Let $w=w_{H}$. Since $\phi$ factors through $\phi(G)$ and $H_{n}(\phi) \neq 0$, we see that $H_{n}\left(\phi(G) ; \mathbb{Z}^{w}\right) \neq 0$ and so $\operatorname{cd} \phi(G)=n$. Therefore $[H: \phi(G)]$ is finite, by the Strebel Theorem. Since $[G: \operatorname{Ker}(\phi)]=|\phi(G)|$ is infinite, the same result implies that $\operatorname{cd} \operatorname{Ker}(\phi)<n$.

If $\phi: G \rightarrow H$ a monomorphism then $[H: \phi(G)]$ is finite, and so the restriction of $w=w_{H}$ to $G$ is $w_{G}$, by the Strebel Theorem again. The restriction homomorphism Res : $H_{n}\left(H ; \mathbb{Z}^{w}\right) \rightarrow H_{n}\left(G ; \mathbb{Z}^{w}\right)$ is an isomorphism [Bi, Section 5.3]. As $\operatorname{deg}(\phi \circ \mathrm{Res})$ is multiplication by the index, the second assertion holds also.

Note also that if $[H: G]$ is finite then $\chi(G)=[H: G] \chi(H)$.

In general, finiteness of the index $[H: \phi(G)]$ does not imply that $\operatorname{deg} \phi \neq 0$. For instance, if $\pi=$ $F(a, b) * \mathbb{Z} F(x, y)$ is the fundamental group of the orientable surface of genus 2 , with presentation $\langle a, b, x, y \mid[a, b]=[x, y]\rangle$, the epimorphism $p: \pi \rightarrow \pi /\langle b, y,[a, x]\rangle \cong \mathbb{Z}^{2}$ factors through the free group $F(a, x)$ and so has degree 0 .

If $\operatorname{deg} \phi \neq 0$ and $\operatorname{Ker}(\phi) \neq 1$ then $\operatorname{Ker}(\phi)$ is not $\mathrm{FP}_{n-1}$ [Bi, Theorem 9.11]. 


\subsection{Mapping tori and Seifert fibred 3-manifolds}

Let $M$ be a closed 3-manifold. Hempel and Jaco showed that a nontrivial finitely generated normal subgroup of infinite index in $\pi=\pi_{1}(M)$ is either $\mathbb{Z}$ or a surface group, and in the latter case the quotient group has two ends [56]. Subsequently, Gabai [44] and Casson and Jungreis [19] showed that if $\pi$ has an infinite cyclic normal subgroup then $M$ is homotopy equivalent to a Seifert fibred 3-manifold. In this section we shall establish the corresponding results for $\mathrm{PD}_{3}$-groups. The full result for the Seifert case rests substantially on the Bowditch Theorem, but we shall first give an earlier argument from [60], which assumes that $v \beta(G)>0$ and uses [31;32] instead of [15] to make the connection with surfaces.

Lemma 8.6. Let $(G, \Omega)$ be a $\mathrm{PD}_{n}$-pair of groups. If $\Omega$ has a finite $G$-orbit then $|\Omega|=2$.

Proof. If $G \cdot \omega$ is finite for some $\omega \in \Omega$ then $\left[G: G_{\omega}\right]<\infty$, and so $G$ is a $\mathrm{PD}_{n-1}$-group. Hence $\left[G: G_{\eta}\right]<\infty$ for all $\eta \in \Omega$, by the Strebel Theorem, and so every orbit is finite.

The exact sequence of coefficients

$$
0 \rightarrow \mathbb{F}_{2} \otimes \Delta(G, \Omega) \rightarrow \mathbb{F}_{2}[\Omega] \rightarrow \mathbb{F}_{2} \rightarrow 0
$$

gives rise to an exact sequence

$$
0 \rightarrow H_{n}\left(G, \Omega ; \mathbb{F}_{2}\right)=\mathbb{F}_{2} \rightarrow H_{n-1}\left(G ; \mathbb{F}_{2}[\Omega]\right) \rightarrow H_{n-1}\left(G ; \mathbb{F}_{2}\right)=\mathbb{F}_{2} .
$$

Since $H_{n-1}\left(G ; \mathbb{F}_{2}[\Omega]\right) \cong \oplus H_{n-1}\left(G_{\eta} ; \mathbb{F}_{2}\right)$ summed over representatives of the orbits, there are at most two orbits.

If $G_{\eta}<G$ then $\Omega$ has at most two $G_{\eta}$-orbits, since $\left(G_{\eta},\left.\Omega\right|_{G_{\eta}}\right)$ is again a $\mathrm{PD}_{n}$-pair of groups. Since the $G_{\eta}$-orbits in $G \cdot \eta$ are singletons, we have $\left[G: G_{\eta}\right]=2$, and $|\Omega|=2$, with $G$ acting nontrivially on $\Omega$. If all boundary components are $G$ then the orbits are singletons, and $\oplus H_{n-1}\left(G_{\eta} ; \mathbb{F}_{2}\right)$ maps onto $H_{n-1}\left(G ; \mathbb{F}_{2}\right)$. Hence we again have $|\Omega|=2$.

When $n=3$ such pairs are realized by $I$-bundles over closed surfaces - the product when $G$ acts trivially on $\Omega$, and the mapping cylinder of a double cover otherwise. We shall say that such pairs are of $I$-bundle type. Note that if $|\Omega|=2$ and $G$ acts nontrivially then $w_{(G, \Omega)}$ is not the canonical orientation character of $G$; the difference is the first Stiefel-Whitney class of the $I$-bundle.

A Seifert 3-manifold group is the fundamental group of an aspherical closed Seifert-fibred 3-manifold.

Theorem 8.7. A $\mathrm{PD}_{3}$-group $G$ is a Seifert 3-manifold group if and only if it has a normal subgroup $C \cong \mathbb{Z}$ and $v \beta(G)>0$.

Proof. An aspherical Seifert fibred 3-manifold is finitely covered by the total space of an $S^{1}$-bundle over an aspherical closed surface, and every such bundle space is Seifert fibred. Thus Seifert 3-manifold groups have such subgroups. Suppose $G$ is such a group. A subgroup of finite index in a group with these properties has them also. On the other hand, if a subgroup of finite index is a Seifert 3-manifold group then so is $G$, by Lemma 8.2. Thus we may assume that $G$ is orientable, $G / G^{\prime}$ is infinite and $C$ is central.

Suppose first that $C$ has finite image in $G / G^{\prime}$. Let $\theta: G \rightarrow \mathbb{Z}$ be an epimorphism, with kernel $K$, and let $t \in G$ represent a generator of $G / K$. Then hd $K=\operatorname{cd} K=2$ and $C \leqslant \zeta K$. Hence either $K$ is abelian or $K^{\prime}$ is a nontrivial free group [Bi, Theorem 8.8].

If $K$ is abelian then $K \cong \mathbb{Z}^{2}$, since hd $K=\operatorname{cd} K=2$. If $K^{\prime} \cong \mathbb{Z}$ then $K \cong \mathrm{Kb}$. In either case, $G / C$ is virtually $\mathbb{Z}^{2}$. 
If $K^{\prime}$ is a nonabelian free group then $C \cap K^{\prime}=1$. Hence $C$ embeds as a submodule of the $\Lambda$-module $M=K / K^{\prime}$, where $\Lambda=\mathbb{Z}[G / K] \cong \mathbb{Z}\left[t, t^{-1}\right]$. Since $G$ is finitely generated as a group, $M$ is finitely generated as a $\Lambda$-module. Let $(t-1)^{s}$ be the highest power of $t-1$ which divides the order ideal of the $\Lambda$-torsion submodule of $M$. Then $C$ embeds in $N=M /(t-1)^{s} M$, which is a finitely generated abelian group, and so $C$ is an abelian group direct summand of $m N$ for some $m \geqslant 1$. The preimage of $m N$ in $G$ is a direct product $K_{1} \cong C \times H$ which is of finite index in $K$ and normal in $G$. Then cd $H=1$, since $\operatorname{cd} C+\operatorname{cd} H=2$. Let $G_{1}$ be the subgroup generated by $K_{1}$ and $t$. Then $G_{1}$ has finite index in $G$ and $G_{1} / C$ is an extension of $K_{1} / \zeta G \cong H$. Hence $\operatorname{cd} G_{1} / C=2$.

If $C$ has infinite image in $G / G^{\prime}$ then $C \cap G^{\prime}=1$, so $G$ is virtually a product $G_{1}=C \times J$. We again have $\mathrm{cd} G_{1} / C=2$.

In each case $\operatorname{cd} G_{1} / C<\infty$ and $H^{2}\left(G_{1} / C ; \mathbb{Z}\left[G_{1} / C\right]\right) \cong \mathbb{Z}$, by an LHS spectral sequence corner argument. Hence $G_{1} / C$ is a $\mathrm{PD}_{2}$-group, and so $G_{1} / C \cong \pi_{1}(F)$, for some aspherical closed surface $F$ [31; 32]. Therefore $G_{1}$ is the fundamental group of an $S^{1}$-bundle over $F$, and so $G$ is a Seifert 3-manifold group.

There is an analogous result for $\mathrm{PD}_{3}$-pairs of groups.

Theorem 8.8. Let $(G, \Omega)$ be a $\mathrm{PD}_{3}$-pair of groups with nonempty boundary. If $G$ has a normal subgroup $A \cong \mathbb{Z}$ then each boundary component is $\mathbb{Z}^{2}$ or $\mathrm{Kb}$, and $(G, \Omega)$ is the peripheral system of a Seifert fibred 3-manifold $M$ with nonempty boundary.

Proof. Since cd $G=2$ and each boundary component $S$ is a $\mathrm{PD}_{2}$-group, we have $A \cap S \neq 1$, for otherwise $\operatorname{cd} A S=3$. Since $A \cap S \cong \mathbb{Z}$ is normal in $S$, it follows that $S \cong \mathbb{Z}^{2}$ or $S \cong \mathrm{Kb}$.

We may assume that $[G: S]=\infty$ for each boundary component $S$, for otherwise the result follows immediately from Lemma 8.6.

Suppose first that $A$ is central in $G$. Then $\zeta G \cong \mathbb{Z}$, and $G^{\prime}$ is a nonabelian free group, by [Bi, Theorem 8.8]. Hence there is an epimorphism $\phi: G \rightarrow \mathbb{Z}$ such that $\left.\phi\right|_{A}$ is injective. Let $N=\operatorname{Ker}(\phi)$, and let $H=A N$. Then $H$ is normal in $G$ and $G / H$ is a finite cyclic group, and $H \cong N \times \mathbb{Z}$. Hence $N$ is a finitely generated free group, and there is a $\operatorname{PD}_{2}$-pair $(N, \mathcal{T})$ such that $\left(H,\left.\Omega\right|_{H}\right) \cong(N, \mathcal{T}) \times \mathbb{Z}$. The pair $(N, \mathcal{T})$ is the peripheral system of a bounded surface $F$ [32].

Let $\alpha$ be the automorphism of $N$ induced by conjugation in $G$ by an element representing a generator of $G / N$. The image of $\alpha$ in $\operatorname{Out}(N)$ has finite order, $k$ say, and preserves the set of conjugacy classes corresponding to the boundary components of $F$, and so is induced by a self-homeomorphism $h$ of $F$, of order $k$, by the Dehn-Nielsen-Baer Theorem [FM, Theorem 8.8]. Let $M$ be the mapping torus of $h$. Then $\pi_{1}(M) \cong N \rtimes_{\alpha} \mathbb{Z} \cong G$, and the peripheral system of $(M, \partial M)$ is isomorphic to $(G, \Omega)$.

If $A$ is not central in $G$ then $\left[G: C_{G}(A)\right]=2$. Let $C=C_{G}(A)$. Since $A \leqslant \zeta C$ and $(G, \Omega)$ is not of $I$-bundle type, $C^{\prime}$ is a nonabelian free group, and $A \cap C^{\prime}=1$. Let $t \in G \backslash C$. Then conjugation in $G$ gives $C^{\mathrm{ab}}$ the structure of a $\mathbb{Z}[t] /\left(t^{2}-1\right)$-module. Let $D$ be the quotient of $C^{\mathrm{ab}} /(t+1) C^{\mathrm{ab}}$ by its torsion subgroup. Then $t$ acts by -1 on $D$, and $A$ maps injectively to $D$. It follows easily that there is an epimorphism $\psi: C \rightarrow \mathbb{Z}$ such that $\left.\psi\right|_{A}$ is injective, and $K=\operatorname{Ker}(\psi)$ is normalized by $t$. Hence $\psi$ extends to an epimorphism $\Psi: G \rightarrow D_{\infty}$, and $G \cong H *_{K} J$, where $[H: K]=[J: K]=2$.

Since $K A \cong K \times A$ has finite index in $G$, it is $\mathrm{FP}_{2}$, and $\left(K A,\left.\Omega\right|_{K A}\right)$ is a $\mathrm{PD}_{3}$-pair of groups. Hence $K$ is a finitely generated free group, and $(K, \Omega / A)$ is a $\mathrm{PD}_{2}$-pair of groups such that $\left(K A,\left.\Omega\right|_{K A}\right) \cong$ $(K, \Omega / A) \times \mathbb{Z}$. Let $h \in H \backslash K$. Then $H$ is also a finitely generated free group, and $h^{2} \in K$. The pair $(H, \Omega / A)$ restricts to $(K, \Omega / A)$, and so is also a $\mathrm{PD}_{2}$-pair of groups [8, Theorem 7.6]. Similarly, $J$ is 
the ambient group of a $\mathrm{PD}_{2}$-pair of groups which restricts to $(K, \mathcal{T})$. These $\mathrm{PD}_{2}$-pairs of groups are the peripheral systems of compact surfaces with nonempty boundary [32]. Hence $(G, \Omega)$ is the peripheral system of a bounded 3-manifold which is the union of two $I$-bundles over bounded surfaces.

This result supports Kropholler's version of the JSJ decomposition for $\mathrm{PD}_{3}$-pairs of groups, considered in Chapter 10.

It follows easily from Theorem 8.7 that if a $\mathrm{PD}_{3}$-group $G$ with nontrivial centre is virtually split over a $\mathrm{PD}_{2}$-group, then it is a Seifert 3-manifold group. However, if we use the Bowditch Theorem, we do not need any hypotheses on $v \beta(G)$ or splitting.

Theorem 8.9. Let $G$ be a $\mathrm{PD}_{3}$-group with a nontrivial $\mathrm{FP}_{2}$ normal subgroup $N$ of infinite index. Then either

(1) $N \cong \mathbb{Z}$ and $G / N$ is virtually a $\mathrm{PD}_{2}$-group, or

(2) $N$ is a $\mathrm{PD}_{2}$-group and $G / N$ has two ends.

Proof. If $N$ is free then $H^{3}(G ; \mathbb{Z}[G]) \cong H^{2}\left(G / N ; H^{1}(N ; \mathbb{Z}[G])\right)$. Since $N$ is finitely generated and $G / N$ is $\mathrm{FP}_{2}$, this is in turn isomorphic to $H^{2}(G / N ; \mathbb{Z}[G / N])^{e(N)-1}$. Since $G$ is a $\mathrm{PD}_{3}$-group we must have $e(N)-1=1$, and so $N \cong \mathbb{Z}$. We then have

$$
H^{2}(G / N ; \mathbb{Z}[G / N]) \cong H^{3}(G ; \mathbb{Z}[G]) \cong \mathbb{Z}^{w_{1}(G)}
$$

Hence $G / N$ is virtually a $\mathrm{PD}_{2}$-group, by the Bowditch Theorem.

Otherwise $\operatorname{cd} N=2$ and so $e(N)=1$ or $\infty$. The Lyndon-Hochschild-Serre spectral sequence gives

$$
H^{2}(G ; \mathbb{Z}[G]) \cong H^{1}(G / N ; \mathbb{Z}[G / N]) \otimes H^{1}(N ; \mathbb{Z}[N]) \cong E(G / N)^{e(N)-1} .
$$

Hence either $e(N)=1$ or $E(G / N)=0$. In the latter case, $H^{3}(G ; \mathbb{Z}[G]) \cong H^{2}(G / N ; \mathbb{Z}[G / N]) \otimes E(N)$ and so $H^{3}(G ; \mathbb{Z}[G])$ is either 0 or infinite dimensional. Therefore $e(N)=1$, and so

$$
H^{3}(G ; \mathbb{Z}[G]) \cong H^{1}(G / N ; \mathbb{Z}[G / N]) \otimes H^{2}(N ; \mathbb{Z}[N]) .
$$

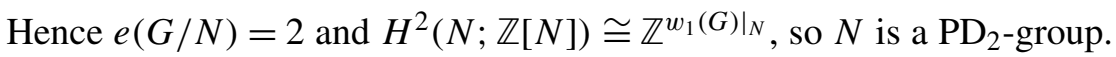

A version of this theorem was first given by Thomas [142], under the additional hypothesis that $G / N$ have an element of infinite order. (This was perhaps the first evidence suggesting that all $\mathrm{PD}_{3}$-groups might be 3-manifold groups.)

Corollary 8.9.1. A $\mathrm{PD}_{3}$-space $P$ is homotopy equivalent to the mapping torus of a self homeomorphism of a closed surface if and only if there is an epimorphism $\phi: \pi_{1}(P) \rightarrow \mathbb{Z}$ with finitely generated kernel.

Proof. If $\operatorname{Ker}(\phi)$ is finite this follows from Theorem 5.8. Otherwise $\pi_{1}(P)$ has one end, so $P$ is aspherical, and we may apply the PD Extension Theorem.

If $\pi_{1}(P)$ is infinite and is a nontrivial direct product, then $P$ is homotopy equivalent to the product of $S^{1}$ with a closed surface. 


\subsection{The groups of aspherical geometric 3-manifolds}

The fundamental groups of 3-manifolds with geometry $\mathbb{E}^{3}, \mathbb{N i l}^{3}$, $\mathbb{S o l}^{3}, \mathbb{M}^{2} \times \mathbb{E}^{1}$ or $\widetilde{\mathbb{S} \mathbb{L}}$ have nontrivial abelian normal subgroups [126]. For four of these, the group is virtually a central extension of a $\mathrm{PD}_{2}^{+}$group by $\mathbb{Z}$, while Sol $^{3}$-groups are virtually extensions of $\mathbb{Z}$ by $\mathbb{Z}^{2}$.

Theorem 8.10. Let $G$ be a $\mathrm{PD}_{3}$-group. Then $G$ is the fundamental group of an aspherical Seifert fibred 3-manifold or a Sol $^{3}$-manifold if and only if $\sqrt{G} \neq 1$. Moreover:

(1) $h(\sqrt{G})=1$ if and only if $G$ is the group of an $\mathbb{U}^{2} \times \mathbb{E}^{1}$ - or $\widetilde{\mathbb{S} \mathbb{L}-m a n i f o l d}$.

(2) $h(\sqrt{G})=2$ if and only if $G$ is the group of a Sol $^{3}$-manifold.

(3) $h(\sqrt{G})=3$ if and only if $G$ is the group of an $\mathbb{E}^{3}$ - or $\mathbb{N i l}^{3}$-manifold.

In all cases, $\sqrt{G}$ is finitely generated.

Proof. The necessity of the conditions is clear.

In general, $h(\sqrt{G}) \leqslant \operatorname{cd} \sqrt{G} \leqslant 3$. Moreover cd $\sqrt{G}=3$ if and only if $[G: \sqrt{G}]$ is finite, by the Strebel Theorem. Hence $G$ is virtually nilpotent if and only if $h(\sqrt{G})=3$. If $h(\sqrt{G})=2$ then $\sqrt{G}$ is locally abelian, and hence abelian. Moreover $\sqrt{G}$ must be finitely generated, for otherwise $\operatorname{cd} \sqrt{G}=3$. Thus $\sqrt{G} \cong \mathbb{Z}^{2}$ and case (2) follows from Theorem 8.9.

Suppose now that $h(\sqrt{G})=1$ and let $C=C_{G}(\sqrt{G})$. Then $\sqrt{G}$ is torsion-free abelian of rank 1, so $\operatorname{Aut}(\sqrt{G})$ is isomorphic to a subgroup of $\mathbb{Q}^{\times}$. Therefore $G / C$ is abelian. If $G / C$ is infinite then $\operatorname{cd} C \leqslant 2$, by the Strebel Theorem. Moreover, $\operatorname{Aut}(\sqrt{G})$ is infinite, so $\sqrt{G} ¥ \mathbb{Z}$. Therefore $C$ is abelian [Bi, Theorem 8.8], and hence $G$ is solvable. But then $G$ is polycyclic, with $h(G)=3[\mathrm{Bi}$, Theorem 9.23], and so $h(\sqrt{G})>1$, which is contrary to our hypothesis. Therefore $G / C$ is isomorphic to a finite subgroup of $\mathbb{Q}^{\times} \cong \mathbb{Z}^{\infty} \oplus \mathbb{Z} / 2 \mathbb{Z}$. We again see that $[G: C] \leqslant 2$. In particular, if $A$ is an infinite cyclic subgroup of $\sqrt{G}$ then $A$ is normal in $G$, and so $G / A$ is virtually a $\mathrm{PD}_{2}$-group, by Theorem 8.9. If $G / A$ is a $\mathrm{PD}_{2}$-group then $G$ is the fundamental group of an $S^{1}$-bundle over a closed surface. In general, a finite torsion-free extension of a Seifert 3-manifold group is again such a group, by Lemma 8.2.

The final assertion is clear.

Case-by-case inspection shows that torsion-free virtually polycyclic groups of Hirsch length $\leqslant 3$ are polycyclic. (This is also so when $n=4$, by the results of [Hi, Chapter 8]. The smallest value of $n$ known to us for which there is a torsion-free, virtually polycyclic group which is not solvable is $n=15$ : there is a torsion-free extension of $I^{*}=\operatorname{PSL}(2,5)$ by $\mathbb{Z}^{15}[116]$.) Henceforth we shall use "polycyclic", rather than "virtually polycyclic", when considering $\mathrm{PD}_{3}$-groups.

A recent and much deeper result is that $\mathbb{Q}^{3}$-groups are virtually extensions of $\mathbb{Z}$ by nonabelian $\mathrm{PD}_{2}^{+}$groups [1]. In this light, the Cannon Conjecture is that every atoroidal $\mathrm{PD}_{3}$-group is virtually such an extension.

This may be reformulated in terms of two other properties. A finitely generated group $G$ which is virtually RFRS (residually finite and rationally solvable) is virtually a semidirect product $H \rtimes \mathbb{Z}$ with $H$ finitely generated if and only if $\beta_{1}^{(2)}(G)=0$ [84]. If such a group $G$ is a $\mathrm{PD}_{3}$-group then it is virtually the group of a mapping torus, by Corollary 8.9.1. Atoroidal mapping tori are hyperbolic, by the Geometrization Theorem. 



\section{CHAPTER 9}

\section{Centralizers, normalizers and ascendant subgroups}

In this chapter we shall consider centralizers and normalizers of subgroups of $\mathrm{PD}_{3}$-groups, $\mathrm{PD}_{3}$-group pairs and open $\mathrm{PD}_{3}$-groups. In $\S 1$ we show that there are essentially three types of pairs $\{C, H\}$ where $C=C_{G}(H)$ is the centralizer of a subgroup of a $\mathrm{PD}_{3}$-group $G$ and $H=C_{G}(C)$ is the maximal subgroup centralized by $C$. We then adapt an argument of Kropholler to show that every strictly increasing chain of centralizers in a $\mathrm{PD}_{3}$-group has length at most 4 . (This bound is best possible.) In particular, Kropholler's pioneering work on JSJ decompositions for $\mathrm{PD}_{n}$-groups with max-c applies to all $\mathrm{PD}_{3}$-groups. (See Chapter 10.) In $\S 3$ and $\S 4$ we consider normalizers of subgroups of $\mathrm{PD}_{3}$-groups that are abelian and $\mathrm{FP}_{2}$; our arguments also apply to subgroups of open $\mathrm{PD}_{3}$-groups. In $\S 5$ we show that if $K$ is an ascendant $\mathrm{FP}_{2}$ subgroup of a $\mathrm{PD}_{3}$-group $G$ then either $N_{G}(K)$ has finite index in $G$ or $K \cong \mathbb{Z}$ and $G$ is polycyclic. In $\S 6$ we summarize work of Elkalla [35] on subnormal subgroups in 3-manifolds groups, which we have not been able to prove in the present setting. Finally, we give short proofs of work of Heil [54] on 3-manifold groups which split over subgroups which are $\mathrm{PD}_{2}$-groups and are properly contained in their normalizers.

\subsection{Centralizers}

If $H$ is a subgroup of $G$ then $\zeta H=H \cap C_{G}(H)$, and $H$ is abelian if and only if $H \leqslant \zeta C_{G}(H)$. Let $H^{\circ}=C_{G}\left(C_{G}(H)\right)$. Then $H \subseteq H^{\circ}$, while $C_{G}\left(H^{\circ}\right)=C_{G}(H)^{\circ}=C_{G}(H)$ and so $H^{\circ \circ}=H^{\circ}$. Thus " $C_{G}(-)$ " is an inclusion-reversing involution on the set of centralizers of subgroups of $G$, and $H^{\circ}$ is the unique maximal subgroup centralized by $C_{G}(H)$.

Lemma 9.1. Let $G$ be a $\mathrm{PD}_{3}$-group such that $\zeta G$ is not cyclic. Then $G \cong \mathbb{Z}^{3}$ or $G \cong \mathrm{Kb} \times \mathbb{Z}$.

Proof. Since $\zeta G \leqslant \sqrt{G}$, we have $h(\sqrt{G}) \geqslant 2$, and so we may apply Theorem 8.10. The centres of $\mathrm{Sol}^{3}$-groups are trivial, while the centres of $\mathrm{Nil}^{3}$-groups are cyclic. Hence $G$ must be virtually $\mathbb{Z}^{3}$, and the lemma follows on examination of the ten posssibilities.

Theorem 9.2. Let $G$ be a $\mathrm{PD}_{3}$-group. If $H$ is a nontrivial subgroup and $C_{G}(H) \neq 1$, we are in one of the following situations.

(1) $C_{G}(H)=H^{\circ}$ and $H^{\circ}$ is a maximal abelian subgroup of rank $\leqslant 2$.

(2) $C_{G}(H) \cong \mathbb{Z}, H^{\circ}$ is not abelian and $\mathrm{cd} H^{\circ}=2$.

(3) $C_{G}(H) \cong \mathbb{Z}, H^{\circ}$ is not abelian and $\left[G: H^{\circ}\right] \leqslant 2$.

(4) $C_{G}(H)$ is not abelian, $\operatorname{cd} C_{G}(H)=2$ and $H^{\circ} \cong \mathbb{Z}$.

(5) $\left[G: C_{G}(H)\right] \leqslant 2, H^{\circ}$ is isomorphic to $\mathbb{Z}$ and is normal in $G$.

(6) $G \cong \mathrm{Kb} \times \mathbb{Z}, C_{G}(H)=\zeta G \cong \mathbb{Z}^{2}$ and $H^{\circ}=G$. 
(7) $G \cong \mathrm{Kb} \times \mathbb{Z}, C_{G}(H)=G$ and $H^{\circ}=\zeta G$.

(8) $G \cong \mathbb{Z}^{3}$.

Proof. If $\zeta H=1$, we have $H \cdot C_{G}(H) \cong H \times C_{G}(H)$. Since $H$ and $C_{G}(H)$ are nontrivial either $\operatorname{cd} H+\operatorname{cd} C_{G}(H)=3$, in which case $H$ is a $\mathrm{PD}_{2}$-group and $C_{G}(H) \cong \mathbb{Z}$, or $H$ and $C_{G}(H)$ are both free, in which case we again have $C_{G}(H) \cong \mathbb{Z}$, since $H \supsetneqq \mathbb{Z}$ and $\mathrm{PD}_{3}$-groups do not contain products of nonabelian free groups [101]. (As observed in Chapter 8, this follows easily from the Algebraic Core Theorem.) Thus we may assume that $H \cap C_{G}(H)=\zeta H \neq 1$.

If $C_{G}(H)$ is abelian then $C_{G}(H)=\zeta H^{\circ}$; if moreover $\operatorname{cd} C_{G}(H)=\mathrm{cd} H^{\circ}$ then $H^{\circ}$ is abelian [Bi, Theorem 8.8]. Thus if $\mathrm{cd} H^{\circ} \leqslant 2$ either (1) or (2) holds. If $\mathrm{cd} H^{\circ}=3$ then $\left[G: H^{\circ}\right]$ is finite, by the Strebel Theorem. Let $B=\bigcap g H^{\circ} g^{-1}$ be the intersection of the conjugates of $H^{\circ}$ in $G$. Then $B$ and $C_{G}(B)$ are normal in $G$ and $\left[C_{G}(B): \zeta B\right] \leqslant[G: B]<\infty$. If $G$ is not virtually $\mathbb{Z}^{3}$ then $\zeta B \cong \mathbb{Z}$, by Lemma 9.1. Hence $C_{G}(B) \cong \mathbb{Z}$, since $G$ is torsion-free. As $C_{G}(H) \leqslant C_{G}(B)$ it follows that $C_{G}(H) \cong \mathbb{Z}$ and is normal in $G$. Hence $H^{\circ}$ is also normal in $G$ and $\left[G: H^{\circ}\right] \leqslant 2$, and so (3) holds.

If $C_{G}(H)$ is free then it is infinite cyclic, since it has nontrivial centre. Thus if $C_{G}(H)$ is nonabelian $\operatorname{cd} C_{G}(H)=2$ or 3. If $\operatorname{cd} C_{G}(H)=2$ then $\operatorname{cd} \zeta C_{G}(H) \leqslant 1$ [Bi, Theorem 8.8], and so $\zeta C_{G}(H) \cong \mathbb{Z}$, since it is nontrivial. If $H^{\circ}=\zeta C_{G}(H)$ then (4) holds. Otherwise, let $J$ be an infinite cyclic subgroup of $C_{G}(H)$ such that $J \cap \zeta C_{G}(H)=1$. Then $J H^{\circ} \cong \mathbb{Z} \times H^{\circ}$ and $\operatorname{cd} J H^{\circ}=3$. Since $\zeta J H^{\circ} \cong \mathbb{Z}^{2}$, it follows that $J H \cong \mathrm{Kb} \times \mathbb{Z}$, by Lemma 9.1 , so $G$ is virtually $\mathbb{Z}^{3}$.

If $\operatorname{cd} C_{G}(H)=3$, arguing as for (3), we see that either $H^{\circ} \cong \mathbb{Z}$ and is normal in $G$, so $\left[G: C_{G}(H)\right] \leqslant 2$ and (5) holds, or $G$ is virtually $\mathbb{Z}^{3}$.

If none of the above five cases apply then $G$ is virtually $\mathbb{Z}^{3}$, and then examination of the ten such groups $G$ shows that nonabelian centralizers have finite index in $G$, so cases (2) and (4) cannot occur, and the remaining possibilities are as in (6), (7) and (8).

In particular, a centralizer $C_{G}(H)$ is never a nonabelian free group. The pairs of cases (2) and (4), (3) and (5), and (6) and (7) are interchanged on applying " $C_{G}(-)$ ", while cases (1) and (8) are invariant under this involution.

Let $G=\pi 3_{1} *_{\mathbb{Z}^{2}} \pi 4_{1}$ be obtained by amalgamating the trefoil and figure-eight knot groups along their peripheral subgroups, and let $H$ be the image of $\pi 3_{1}$ and $\widetilde{H} \cong \mathbb{Z}$ be the subgroup generated by a nontrivial element of $\pi 4_{1}$ which is not conjugate to a peripheral element. Then $C_{G}(\widetilde{H})=\widetilde{H}$ and $C_{G}(H) \cong \mathbb{Z}=\zeta H$ are examples representing cases (1) and (2). The common $\mathbb{Z}^{2}$ subgroup in the amalgamation of two copies of $\pi 4_{1}$ gives an example of rank 2 in case (1). If $C$ is a $\mathrm{PD}_{2}$-group with trivial centre then $G=C \times \mathbb{Z}$ gives an example for cases (3) and (5).

Theorem 9.3. Let $G$ be an atoroidal $\mathrm{PD}_{3}$-group and $h \in G \backslash\{1\}$. Then $C=C_{G}(h)$ is a maximal abelian subgroup, and has rank 1.

Proof. If [ $G: C]<\infty$ then $C$ is a $\mathrm{PD}_{3}$-group of Seifert type, and so $G$ is not atoroidal. If cd $C=2$ then either $C \cong \mathbb{Z}$ or $\mathrm{Kb}$, or $C^{\prime}$ is a nonabelian free group, or $C$ is an abelian group of rank 1 . The first three possibilities are again inconsistent with $G$ being atoroidal. Finally, if $\operatorname{cd} C \leqslant 1$ then $C \cong \mathbb{Z}$, since it is a free group with nontrivial centre.

If $C \leqslant D$, where $D$ is an abelian group, then $h d=d h$ for all $d \in D$, since $h \in C$. Hence $D \leqslant C$ and so $C$ is maximal abelian. 
We do not yet know whether $C_{G}(h)$ must be finitely generated.

Theorem 9.4. Let $G$ be a $\mathrm{PD}_{3}$-group. If $C_{G}(K)$ is finitely generated for all infinite cyclic subgroups $K<G$ then $C_{G}(H)$ is finitely generated for all subgroups $H<G$, and every abelian subgroup of $G$ is finitely generated.

Proof. We may assume that $G$ is not polycyclic, since all subgroups of such groups are finitely generated. The first assertion follows immediately from Theorem 9.2 if $H \cong \mathbb{Z}^{2}$ or if $H$ is nonabelian, for then $C_{G}(H) \cong \mathbb{Z}^{2}$ or $\mathbb{Z}$ or 1 , respectively. Suppose that $H$ is abelian of rank 1 , but is not finitely generated. Let $K$ be an infinite cyclic subgroup of $H$. Then $K \leqslant H \leqslant C_{G}(K)$, so $H / K \leqslant C_{G}(K) / K$, and $C_{G}(K)$ is finitely generated, by hypothesis. If $\operatorname{cd} C_{G}(K)=2$ then $C_{G}(K) / K$ is virtually free [Bi, Theorem 8.4], while if $\operatorname{cd} C_{G}(K)=3$ then $C_{G}(K) / K$ is virtually a $\mathrm{PD}_{2}$-group, by the Bowditch Theorem. In either case the torsion subgroups of $C_{G}(K) / K$ are finite, and so $H$ must be finitely generated, contrary to the assumption.

Castel showed that if every abelian subgroup of $G$ is finitely generated then $G$ has no nontrivial infinitely divisible elements, and hence that the three conditions in Theorem 9.4 are equivalent [20, Theorem 1]. (We shall return to this in Chapter 10.) All known abelian subgroups of $\mathrm{PD}_{3}$-groups are finitely generated.

\subsection{The maximum condition on centralizers}

Kropholler [96] showed that if $M$ is a 3-manifold, every strictly increasing sequence of centralizers $1 \leqslant C_{0}<C_{2}<\ldots C_{n}=G$ in $G=\pi_{1}(M)$ has length $n$ at most 16 , while if $M$ is aspherical, $n \leqslant 11$. (Note that in [96] such a sequence is said to have length $n+1$.) Using the JSJ decomposition theory of Scott and Swarup [127], Castel has shown that every such sequence of centralizers in a $\mathrm{PD}_{3}$-group is finite [20]. We shall reprove and sharpen this result. Our argument follows [96, Lemma 8] closely, using the geometry of plane isometry groups instead of imbedding flat 3-manifold groups in matrix groups, and using the Algebraic Core Theorem rather than the 3-manifold version. In particular, it does not use any JSJ decomposition theory.

Theorem 9.5. Let $G$ be a $\mathrm{PD}_{3}$-group. Then every strictly increasing sequence of centralizers in $G$ has length at most 4.

Proof. Suppose first that $G$ has an infinite cyclic normal subgroup $N$. Then $G / N$ is virtually a $\mathrm{PD}_{2}$-group, by Theorem 8.9. We may assume that $G / N$ has no finite normal subgroup, and hence is isomorphic to a discrete cocompact group of isometries of the euclidean or hyperbolic plane [33]. Proper centralizers in such groups are maximal abelian: cyclic, $(\mathbb{Z} / 2 \mathbb{Z})^{2}, \mathbb{Z} / 2 \mathbb{Z} \oplus \mathbb{Z}$ or $\mathbb{Z}^{2}$, the latter in the euclidean case only. It follows easily that chains of centralizers in $C_{G}(N)$ have length at most 2 , and hence that chains of centralizers in $G$ have length at most 3.

Suppose now that $G$ has no infinite cyclic normal subgroup, and that $G$ has a centralizer sequence $1<$ $C_{1}<\cdots<C_{5}=G$ of length 5. Then either $C_{3}$ is nonabelian or $C_{G}\left(C_{3}\right)$ is nonabelian or $C_{3}=C_{G}\left(C_{3}\right)$ is self-centralizing and maximal abelian, so $C_{G}\left(C_{2}\right)$ is nonabelian. In each case either $C_{3}<C_{4}$ or $C_{G}\left(C_{2}\right)<$ $C_{G}\left(C_{1}\right)$ are proper nonabelian centralisers. (This argument is from [96, Lemma 7].) Relabelling, we let $C_{1}<C_{2}$ denote such a nested pair of proper centralizers. Since $G$ has no infinite cyclic normal subgroup, $\left[G: C_{2}\right]$ is infinite. In particular, $Z_{i}=C_{G}\left(C_{i}\right)=\zeta C_{i} \cong \mathbb{Z}$ for $i \leqslant 2$, by Theorem 9.2. Moreover $C_{1}$ is not 
normal in $C_{2}$, for otherwise $\zeta C_{1} \cong \mathbb{Z}$ would be central in $C_{2}$. Let $H$ be a finitely generated subgroup of $C_{2}$ such that $Z_{1}<H \cap C_{1}<H$ and $\left(H \cap C_{1}\right) / Z_{1}$ is infinite and nonabelian. Then $Z_{2} \leqslant \zeta H<Z_{1}$ and so $H$ has one end, since it has nontrivial centre but is not virtually $\mathbb{Z}$. Since cd $H \leqslant 2$ and $\zeta H \cong \mathbb{Z}, H / \zeta H$ is virtually free [ $\mathrm{Bi}$, Theorem 8.4$]$. Hence $H$ is finitely presentable, and so it is the ambient group of a $\mathrm{PD}_{3}$-pair of groups $(H, \mathcal{T})$, by the Algebraic Core Theorem. Doubling $H$ along $\mathcal{T}$ gives a $\mathrm{PD}_{3}$-group $D H$ with nontrivial centre [8]. Proper centralizers in $D H / \zeta D H$ are abelian, as in the first paragraph. But $\left(H \cap C_{1}\right) / \zeta H$ is nonabelian and centralizes the nontrivial subgroup $Z_{1} / \zeta H$. Thus every centralizer sequence in $G$ has length at most 4.

This proof of Theorem 9.5 uses the Algebraic Core Theorem. The first proof that $\mathrm{PD}_{3}$-groups have max-c used Scott-Swarup JSJ decomposition theory [20]. However, Theorem 9.5 shows that Kropholler's earlier work on JSJ decompositions of $\mathrm{PD}_{3}$-groups [94] applies in full generality.

The bound 4 is best possible. For let $M$ be the 3-manifold obtained by replacing a neighbourhood of an $S^{1}$-fibre in $S^{1} \times S^{1} \times S^{1}$ by the exterior of a hyperbolic knot $K$. Then $G=\pi_{1}(M) \cong(\mathbb{Z} \times F(2)) * \mathbb{Z}^{2} \pi K$, and $G$ has a centralizer sequence

$$
1<\mathbb{Z}<\mathbb{Z}^{2}<\mathbb{Z} \times F(2)<G .
$$

(It follows easily from the argument for Theorem 9.5 that the middle term of any centralizer sequence of length 4 must be a self-centralizing maximal abelian subgroup.)

If the fundamental group $G$ of an open 3-manifold is not finitely generated and has nontrivial centre, then $G$ is torsion-free and either $G$ is abelian of rank 1 or $\zeta G \cong \mathbb{Z}$ [124]. The arguments of Theorem 9.2 extend easily to show that if $G$ is an open $\mathrm{PD}_{3}$-group and $H$ is a nontrivial subgroup with nontrivial centralizer then either (1), (2) or (4) holds. The argument of the second paragraph of the proof of Theorem 9.5 applies almost without change to show that any centralizer sequence in an open $\mathrm{PD}_{3}$-group has length at most 4 . This estimate is again best possible, for the fundamental group $\mathbb{Z} \rtimes F(2)$ of the nontrivial $S^{1}$-bundle over the once-punctured torus has a centralizer sequence of length 4 . In particular, if $(G, \Omega)$ is a $\mathrm{PD}_{3}$-pair of groups then $G$ has max-c.

If $\theta$ is an automorphism of finite order of a finitely generated free group $F$, the semidirect product $F \rtimes_{\theta} \mathbb{Z}$ has cohomological dimension 2 and nontrivial centre. It is easy to find such groups with proper nonabelian centralizers. The following example suggests that some additional topological input (such as the appeal to [33]) may be necessary for the final step of Kropholler's argument.

Example. Let $F$ be the free group with basis $\left\{x_{i} \mid i \geqslant 2\right\}$, and let $\theta: F \rightarrow F$ be the automorphism determined by $\theta\left(x_{i}\right)=x_{i+1}$ if $i+1$ is not a power of 2 , and $\theta\left(x_{2^{k}-1}\right)=x_{2^{k-1}}$ for $k \geqslant 1$. The subgroup $F_{n}$ generated by $\left\{x_{i} \mid 2 \leqslant i<2^{n}\right\}$ is invariant under $\theta$, for each $n \geqslant 2$, and $F=\bigcup_{n \geqslant 2} F_{n}$. Let $B_{n}=F_{n} \rtimes_{\theta} \mathbb{Z}$. Then $\zeta B_{n}=\left\langle t^{2^{n-1}}\right\rangle$, and $B_{n}$ is the fundamental group of a bounded aspherical 3-manifold. Hence $H=$ $F \rtimes_{\theta} \mathbb{Z}=\bigcup_{n \geqslant 2} B_{n}$ is an open $\mathrm{PD}_{3}$-group, and is coherent (although not finitely generated). The sequence of centralizers $H_{n}=C_{H}\left(\zeta B_{n}\right)$ is properly increasing, with union $H$, and so $H$ does not have max-c.

\subsection{Normalizers of rank 1 abelian subgroups}

Let $G$ be a $\mathrm{PD}_{3}$-group and $A<G$ an infinite cyclic subgroup. Then $\left[N_{G}(A): C_{G}(A)\right] \leqslant 2$, since $\operatorname{Aut}(A)=\{ \pm 1\}$. If $C_{G}(A)$ is finitely generated then either $N_{G}(A)=C_{G}(A) \cong \mathbb{Z}$, or $\operatorname{cd} N_{G}(A) \leqslant 2$ and $N_{G}(A) / A$ is virtually free, or $\left[G: N_{G}(A)\right]<\infty$. In this last case $G$ is the fundamental group of an 
aspherical closed Seifert fibred 3-manifold and either $A$ is normal in $G$ or $G$ is polycyclic.

If $C=C_{G}(A)$ is not finitely generated then it is a rank 1 abelian group [20, Theorem 1] (see Theorem 10.11), and so is maximal abelian, by Theorem 9.2. The quotient $N_{G}(C) / C$ is isomorphic to a subgroup of $\operatorname{Aut}(C) \leqslant \mathbb{Q}^{\times}$, and hence is free abelian (since $N_{G}(C)$ is torsion-free). Hence $\left[G: N_{G}(C)\right]$ is infinite. For otherwise $G$ would be virtually solvable and hence polycyclic [Bi, Theorem 9.23], and so every subgroup of $G$ would be finitely generated. Suppose that $C<N_{G}(C)$ and choose elements $c \in C \backslash\{1\}$ and $n \in N_{G}(C) \backslash C$. The subgroup $H$ generated by $\{c, n\}$ is solvable and $\operatorname{cd} H \leqslant 2$. Since $H$ is not virtually abelian, we have $H \cong \mathrm{BS}(1, q)=\mathbb{Z} *_{q}$ for some $q \notin\{0, \pm 1\}$ [48]. As $H$ is finitely presentable and has one end, it is the ambient group of a $\mathrm{PD}_{3}$-pair of groups, by the Algebraic Core Theorem. But this is impossible (see $\S 5$ of Chapter 10 ), and so $C=N_{G}(C)$. In particular, no such subgroup $A$ is normal in $G$.

Suppose now that $G$ is an open $\mathrm{PD}_{3}$-group. If $A$ is an infinite cyclic subgroup of $G$ then either $N_{G}(A) \cong \mathbb{Z}$ or $\operatorname{cd} N_{G}(A)=2$ and $N_{G}(A) / A$ is locally virtually free. However, if $G=\mathbb{Z} \times F(\infty)$ and $A=\zeta G$ then $N_{G}(A)$ is not finitely generated. If $C$ is an abelian subgroup of $G$ which is not finitely generated then $C_{G}(C)$ is maximal abelian of rank 1. The argument given for the closed case again implies that $N_{G}(C)=C_{G}(C)$. Every rank 1 abelian group is trivially an open $\mathrm{PD}_{3}$-group, as it has no finitely generated subgroup with one end. (In fact such groups are the fundamental groups of open 3-manifolds given by increasing unions of solid tori $S^{1} \times D^{2}$.) Is there a nonabelian open $\mathrm{PD}_{3}$-group with a non-finitely generated abelian subgroup?

\subsection{Normalizers of other $\mathrm{FP}_{2}$ subgroups}

Let $G$ be a $\mathrm{PD}_{3}$-group and $A<G$ an abelian subgroup of rank $r>1$. Then $2 \leqslant r \leqslant \operatorname{cd} A \leqslant 3$, and therefore either $r=\operatorname{cd} A=2$ or $\operatorname{cd} A=3$. In either case, $A$ is finitely generated. The determination of $N_{G}(A)$ follows easily from the next lemma.

Lemma 9.6. Let $G$ be a $\mathrm{PD}_{3}$-group with subgroups $H$ and $J$ such that $H$ is $\mathrm{FP}_{2}$, has one end and is normal in $J$. Then either $[J: H]$ or $[G: J]$ is finite.

Proof. Suppose that $[J: H]$ and $[G: J]$ are both infinite. Since $H$ has one end it is not free and so $\operatorname{cd} H=\operatorname{cd} J=2$, by the Strebel Theorem. Hence there is a free $\mathbb{Z}[J]$-module $W$ such that $H^{2}(J ; W) \neq 0$ [Bi, Proposition 5.1]. Since $H$ is $\mathrm{FP}_{2}$ and has one end, $H^{q}(H ; W)=0$ for $q=0$ or 1 and $H^{2}(H ; W)$ is an induced $\mathbb{Z}[J / H]$-module. Since $[J: H]$ is infinite, $H^{0}\left(J / H ; H^{2}(H ; W)\right)=0$ [Bi, Lemma 8.1]. The LHSSS for $J$ as an extension of $J / H$ by $H$ now gives $H^{r}(J ; W)=0$ for $r \leqslant 2$, which is a contradiction.

If $G$ is a $\mathrm{PD}_{3}$-group and $K$ is an $\mathrm{FP}_{2}$ subgroup of $G$ which is not free then either $\left[G: N_{G}(K)\right]$ is finite or $\left[N_{G}(K): K\right]$ is finite, by Lemma 9.6 (in the one-ended case) and the following result.

Theorem 9.7. Let $G$ be a $\mathrm{PD}_{3}$-group with an $\mathrm{FP}_{2}$ subgroup $K$ which is a nontrivial free product but is not free. Then $\left[N_{G}(K): K\right]$ is finite and $C_{G}(K)=1$.

Proof. Since $K$ has infinitely many ends, $\operatorname{cd} N_{G}(K) \leqslant 2$, by a spectral sequence corner argument and Poincaré duality, and since $K$ is not free, $\operatorname{cd} K=\operatorname{cd} N_{G}(K)=2$. Hence $\left[N_{G}(K): K\right]$ is finite [Bi, Corollary 8.6]. Since nontrivial free products have trivial centre and $G$ is torsion-free it follows that $C_{G}(K)=1$. 
Theorem 9.8. Let $G$ be a $\mathrm{PD}_{3}$-group. If $F$ is a finitely generated nonabelian free subgroup of $G$ then $N_{G}(F)$ is finitely generated and $N_{G}(F) / F$ is finite or virtually $\mathbb{Z}$. Moreover $C_{G}(F) \cong \mathbb{Z}$ or 1 .

Proof. Since $F$ is not cyclic, $N_{G}(F)$ cannot be a $\mathrm{PD}_{3}$-group, by Theorem 8.9 , and so cd $N_{G}(F) \leqslant 2$. Let $H$ be a finitely generated subgroup of $N_{G}(F)$ which contains $F$. Then $H / F$ is virtually free [Bi, Theorem 8.4], and so $H$ is finitely presentable. In particular, $\chi(H)=\chi(F) \chi(H / F)$. Now $\chi(H) \leqslant 0$, by the Algebraic Core Theorem and Lemma 3.1. Since $\chi(F)<0$ this is only possible if $\chi(H / F) \geqslant 0$. Therefore either $H / F$ is finite, in which case $H$ is free and $[H: F]=\chi(F) / \chi(H) \leqslant|\chi(F)|$, or $H / F$ is virtually $\mathbb{Z}$ and $H$ has one end.

If $N_{G}(F) / F$ is locally finite it is finite (and $\left[N_{G}(F): F\right] \leqslant|\chi(F)|$ ). Hence we may assume that $N_{G}(F)$ is the union of an increasing sequence $N_{0}=F<N_{1} \leqslant \cdots$ of finitely generated subgroups with $N_{i} / F$ virtually $\mathbb{Z}$, for $i \geqslant 1$. For each $i \geqslant 1$ the group $N_{i}$ is $\mathrm{FP}_{2}, \operatorname{cd} N_{i}=2, H^{s}\left(N_{i} ; \mathbb{Z}\left[N_{i}\right]\right)=0$ for $s \leqslant 1$ and $\left[N_{i+1}: N_{i}\right]$ is finite. Hence $N_{G}(F)$ is finitely generated, by the Gildenhuys-Strebel Theorem, and so $N_{G}(F) / F$ is virtually $\mathbb{Z}$.

Since $C_{G}(F) \cap F=\zeta F$ is trivial, projection to $N_{G}(F) / F$ is injective on $C_{G}(F)$, and so $C_{G}(F)$ is infinite cyclic or trivial.

After replacing $F$ by a finite extension $\hat{F}$ with $[\hat{F}: F]$ maximal, if necessary, we may assume that either $F=N_{G}(F)$ or that $N_{G}(F) / F \cong \mathbb{Z}$ or $D_{\infty}$. Both possibilities can occur; if $G=K \times \mathbb{Z}$, where $K$ is a $\mathrm{PD}_{2}$-group and $\chi(K)<0$, then any noncyclic 2-generator subgroup $F$ of $K$ is free and its normalizer in $G$ is the direct product $F \times \mathbb{Z}$. The fundamental group of a closed hyperbolic 3-manifold $M$ is atoroidal. If moreover $\beta_{1}(M ; \mathbb{Z}) \geqslant 3$ then $G=\pi_{1}(M)$ has noncyclic 2-generator subgroups, and every such subgroup $F$ has infinite index. Hence $F$ is free [JS, Theorem VI.4.1], and $F=N_{G}(F)$, for otherwise $\left[N_{G}(F): F\right]$ would be infinite and $G$ would contain a copy of $\mathbb{Z}^{2}$, by the argument of the Corollary below.

Can the fact that $\chi(H) \leqslant 0$ be proven directly without appeal to the Algebraic Core Theorem?

Corollary 9.8.1. If $G$ is an atoroidal $\mathrm{PD}_{3}$-group and $F$ is a finitely generated nonabelian free subgroup of $G$ then $\left[N_{G}(F): F\right]$ is finite.

Proof. If $\left[N_{G}(F): F\right]$ is infinite then $N=N_{G}(F)$ is $\mathrm{FP}_{2}$ and has one end. Hence it is the ambient group of a $\mathrm{PD}_{3}$-pair of groups, by the Algebraic Core Theorem. Since $N / F$ is virtually $\mathbb{Z}$, cd $N=2$, so the boundary is nonempty, and since $\chi(N)=\chi(F) \chi(N / F)=0$ the boundary components are copies of $\mathbb{Z}^{2}$ or $\mathrm{Kb}$, contrary to the hypothesis that $G$ be atoroidal.

If $G$ has no $\mathrm{PD}_{2}$-subgroup of genus less than $k$, and $H$ is a one-ended $\mathrm{FP}_{2}$ subgroup of infinite index in $G$, then the Algebraic Core Theorem implies that $\chi(H) \leqslant 1-k$. In the 3-manifold case every finitely generated subgroup $H$ of infinite index in a 3-manifold group has a finite 2-dimensional $K(H, 1)$ space, and there is a stronger result [5]. For if $K(H, 1)$ is a finite 2-complex and $\chi(H) \leqslant 1-k$, then $H$ has deficiency at least $k$. Now a $k$-generator group with a finite presentation of deficiency $k$ is free [Ro, 14.1.7]. Therefore, if $H$ is generated by at most $k$ elements then $H$ is free. In particular, if $G$ is atoroidal then all 2-generator subgroups of infinite index and with finite 2-dimensional classifying spaces are free.

If $F \leqslant G$ are as in Theorem 9.8 and $\left[N_{G}(F): F\right]$ is infinite, then $N_{G}(F)$ is the ambient group of a $\mathrm{PD}_{3}$-pair of groups with nonempty boundary and a nontrivial finitely generated normal subgroup, by the Algebraic Core Theorem. 
Theorem 9.9. Let $(G, \Omega)$ be a $\mathrm{PD}_{3}$-pair of groups with nonempty boundary, and let $N$ be a nontrivial finitely generated normal subgroup of infinite index in $G$. Then:

(1) $N$ is free and $G / N$ is virtually free of positive rank.

(2) If $S$ is a boundary component of $(G, \Omega)$ then $S \cong \mathbb{Z}^{2}$ or $S \cong \mathrm{Kb}$, and $S \cap N \cong \mathbb{Z}$.

(3) If $N \cong \mathbb{Z}$ there is a subgroup $H$ of finite index in $G$ and a $\mathrm{PD}_{2}$-pair of groups $(F, \mathcal{T})$ such that $\left(H,\left.\Omega\right|_{H}\right) \cong \mathbb{Z} \times(F, \mathcal{T})$.

(4) If $\operatorname{rank}(N)>1$ then $G / N$ has two ends and $\operatorname{rank}(N) \geqslant|\Omega / G|-1$.

Proof. Since $G$ has a finitely generated infinite normal subgroup of infinite index, $\beta_{1}^{(2)}(G)=0$, and so $\chi(G) \geqslant 0$. Hence $\chi(G)=0$, and so $N$ is $\mathrm{FP}_{2}$ [88].

The first assertion follows from [Bi, Corollary 8.6], since $\operatorname{cd} G=2$. It follows that $\chi(N)$ and $\chi(G / N)$ are well-defined, and nonpositive. Hence $\chi(G)=\chi(N) \chi(G / N) \geqslant 0$. But $2 \chi(G)=\sum \chi\left(G_{\eta}\right) \leqslant 0$, where the sum is over $G$-orbits $G \eta$ in $\Omega$, by Lemma 3.1. Hence $\chi(G)=0$ and either $\chi(N)=0$ or $\chi(G / N)=0$. Moreover $\chi(S)=0$ and so $S \cong \mathbb{Z}^{2}$ or $S \cong \mathrm{Kb}$, for all boundary components $S$. Each of the subgroups $S \cap N$ is free, and is nontrivial, for otherwise the $\mathrm{PD}_{2}$-group $S$ would map injectively to the virtually free group $G / N$. Therefore $S \cap N \cong \mathbb{Z}$, for all such $S$.

If $N \cong \mathbb{Z}$ then (on passing to a subgroup of finite index in $G$, if necessary) we may assume that $N$ is central in $G$ and that $G / N$ is free. Then $G \cong N \times(G / N)$. Choose a splitting $p: G \rightarrow N$. Then $F=\operatorname{Ker}(p)$ is a finitely generated free group and $F \cap S \cong \mathbb{Z}$, for each boundary component $S$. Let $K=N \cap \bigcap S$ and $H=K \cdot F \cong K \times F$. Then $K \cong \mathbb{Z}$ and $\operatorname{cd} F \leqslant \operatorname{cd} G<\infty$, so $(F, \Omega / N)$ is a $\mathrm{PD}_{2}$-pair of groups and $\left(H,\left.\Omega\right|_{H}\right) \cong \mathbb{Z} \times(F, \Omega / N)$, by [8, Theorem 7.3].

If $N$ has rank $>1$ then $\chi(N)<0$, so $\chi(G / N)=0$ and $G / N$ is virtually $\mathbb{Z}$. The coset space $G / N S$ is finite, for each boundary component $S$. Let $T_{S} \subset G$ be a transversal to this coset space. Then $N$ is the ambient group for a $\mathrm{PD}_{2}$-pair of groups $(N, \mathcal{N})$, for which the boundary components are represented by the infinite cyclic subgroups $t(N \cap S) t^{-1}$, taken over all $t \in T_{S}$ and boundary components $S$. (See [8, Theorem 6.2].) Hence $\operatorname{rank}(N) \geqslant|\mathcal{N}|-1 \geqslant|\Omega / G|-1$.

If $N \cong \mathbb{Z}$ then $(G, \Omega)$ is the peripheral system of a Seifert fibre space $M$ with nonempty boundary. If $N$ has rank $>1$ then $G$ has a normal subgroup $K$ which contains $N$ as a subgroup of finite index and such that $G / K$ is isomorphic to $\mathbb{Z}$ or $D_{\infty}$, and so $G$ is virtually the group of a surface bundle over a circle. (As in the closed case, it is easy to construct examples with $G / N \cong D_{\infty}$ from unions of twisted $I$-bundles over bounded surfaces.) See Theorem 8.8 .

Let $M$ be the mapping torus of a rotation of the thrice-punctured sphere which permutes the punctures. Then $G=\pi_{1}(M)$ has the presentation $\left\langle x, t \mid x t x t x=t^{2}\right\rangle$, and $S=\pi_{1}(\partial M)=\left\langle x, t^{3}\right\rangle \cong \mathbb{Z}^{2}$. The normal subgroup $N=\langle\langle x\rangle$ is free of rank $2>|\partial M|-1=0$, and the boundary components of $\mathcal{N}$ are represented by $S \cap N, t(S \cap N) t^{-1}$ and $t^{2}(S \cap N) t^{-2}$ (corresponding to the cosets in $G / N \sigma(S) \cong \mathbb{Z} / 3 \mathbb{Z}$ ).

\subsection{Ascendant $\mathrm{FP}_{2}$ subgroups}

A subgroup $K$ of a group $G$ is ascendant if there is a series

$$
K=K_{0}<K_{1}<\cdots<K_{\beth}=G
$$

of subgroups indexed by ordinals $\leqslant \beth$, such that $K_{\alpha}$ is normal in $K_{\alpha+1}$ if $\alpha<\beth$, and $K_{\beta}=\bigcup_{\alpha<\beta} K_{\alpha}$ 
for all limit ordinals $\beta \leqslant \beth$. If $\beth$ is finite, $K$ is subnormal in $G$. Such ascendant series are well suited to arguments by transfinite induction. For instance, it is easily seen that $\sqrt{K} \leqslant \sqrt{K_{\alpha}}$, for all $\alpha \leqslant \beth$.

We may extend [Bi, Corollary 8.6] to ascendant $\mathrm{FP}_{2}$ subgroups.

Lemma 9.10. Let $G$ be a group such that $\mathrm{cd} G=2$. If $K$ is an ascendant $\mathrm{FP}_{2}$ subgroup of $G$ then either $[G: K]$ is finite or $K$ is free.

Proof. We may assume that $K$ is not free, and so $\operatorname{cd} K=\operatorname{cd} G=2$. Suppose first that $K$ is normal in $G$. Then $G / K$ is locally finite [Bi, Corollary 8.6], and so $G$ is the increasing union of a (possibly finite) sequence of $\mathrm{FP}_{2}$ subgroups $U_{i}$ such that $U_{0}=K$ and $\left[U_{i+1}: U_{i}\right]$ is finite, for all $i \geqslant 0$. It follows from the Kurosh subgroup theorem that if $U \leqslant V$ are finitely generated groups and $[V: U]$ is finite, then $V$ has strictly fewer indecomposable factors than $U$, unless both groups are indecomposable [124, Lemma 1.4]. Hence if $K$ is a nontrivial free product then $[G: K]$ is finite. Otherwise $K$ has one end, and so $H^{s}\left(U_{i} ; \mathbb{Z}\left[U_{i}\right]\right)=0$ for $s \leqslant 1$ and $i \geqslant 0$. Since $K$ is $\mathrm{FP}_{2}$, the successive indices are finite and $\operatorname{cd} U_{i}=2=\operatorname{cd} G$ for all $i \geqslant 0$, we see that the union is finitely generated, by the Gildenhuys-Strebel Theorem. Hence the sequence terminates and $[G: K]$ is again finite.

If $K=K_{0}<K_{1}<\cdots<K_{\beth}=G$ is an ascendant series then $\left[K_{\alpha+1}: K_{\alpha}\right]<\infty$ for all $\alpha$, by the argument just given. Let $\omega$ be the union of the finite ordinals in $\beth$. Then $\bigcup_{\alpha<\omega} K_{\alpha}$ is finitely generated, by the Gildenhuys-Strebel Theorem, and so $\omega$ is finite. Hence the series is finite, and so $[G: K]<\infty$.

This lemma indicates how the interaction between the Gildenhuys-Strebel Theorem and the Strebel Theorem may be the key to showing that ascendant $\mathrm{FP}_{2}$ groups are virtually normal.

It follows from Lemma 9.6, Theorem 9.7 and Theorem 9.8 that if $H$ is an $\mathrm{FP}_{2}$ subgroup of a $\mathrm{PD}_{3}$-group $G$ and $N_{G}(H)$ is not finitely generated, then $H \cong \mathbb{Z}$. Our next theorem extends this result.

Theorem 9.11. Let $G$ be an open $\mathrm{PD}_{3}$-group. If $K$ is a nontrivial $\mathrm{FP}_{2}$ ascendant subgroup of $G$, then either

(1) $G$ is $\mathrm{FP}_{2}$ and $\left[G: N_{G}(K)\right]$ is finite, or

(2) $K$ is isomorphic to $\mathbb{Z}$ and normal in $G$.

Proof. Let $K=K_{0}<K_{1}<\cdots<K_{\beth}=G$ be an ascendant series. We may assume that $[G: K]=\infty$, and so $K$ is free, by Lemma 9.10. Suppose first that $K$ is nonabelian. If $\left[K_{\alpha}: K\right]$ is finite then $\alpha \leqslant|\chi(K)|$, so $j=\min \left\{\alpha \mid\left[K_{\alpha+1}: K\right]=\infty\right\}$ is a finite ordinal. Then $K_{j}$ is a finitely generated nonabelian free group, and so $K_{j+1} / K_{j}$ is virtually $\mathbb{Z}$, by Theorem 9.8. Hence $K_{j+1}$ is $\mathrm{FP}_{2}$ and cd $K_{j+1}=2$. Since $K_{j+1}$ is ascendant, $\left[G: K_{j+1}\right]$ is finite, by Lemma 9.10, and so $G$ is $\mathrm{FP}_{2}$ also. Since $K_{j}$ is finitely generated, it has only finitely many subgroups of index $\left[K_{j}: K\right]$. Therefore $N_{G}(K) \cap K_{j+1}$ has finite index in $K_{j+1}$ and so $\left[G: N_{G}(K)\right]$ is finite.

If $K \cong \mathbb{Z}$ then $K \leqslant \sqrt{K_{\alpha}}$, for all $\alpha \leqslant \beth$, and so $K \leqslant \sqrt{G}$. Moreover cd $\sqrt{G} \leqslant \operatorname{cd} G \leqslant 2$, and so $\sqrt{G}$ is a torsion-free abelian group. If $\sqrt{G} \cong \mathbb{Z}$ then $G$ acts on $\sqrt{G}$ through $\{ \pm 1\}$, and so $K$ is normal in $G$. If $\sqrt{G} \cong \mathbb{Z}^{2}$ then $[G: \sqrt{G}]$ is finite, by Lemma 9.10 , so $G \cong \mathbb{Z}^{2}$ or $G \cong \mathrm{Kb}$. Otherwise $\sqrt{G}$ is a nonfinitely generated rank 1 abelian group. In the latter case $\sqrt{G}=C_{G}(\sqrt{G})$ [Bi, Theorem 8.8], and $\operatorname{Aut}(\sqrt{G})$ is abelian, so $G$ is solvable. If $G$ is finitely generated it is $\mathrm{FP}_{2}$ and one-ended [48], and so is the ambient group of a $\mathrm{PD}_{3}$-pair of groups $(G, \mathcal{S})$ (by the definition of open $\mathrm{PD}_{3}$-group). Since cd $G<3$ the set $\mathcal{S}$ is nonempty, and so $\mathbb{Z}^{2}$ is a subgroup of $G$. It follows easily that $G \cong \mathbb{Z}^{2}$ or $G \cong \mathrm{Kb}$, contradicting the 
hypothesis on $\sqrt{G}$. Hence $G$ is not finitely generated, and so is a rank 1 abelian group [48]. In this case $K$ is again normal in $G$.

The corresponding result for normal subgroups of the fundamental group of an open 3-manifold is due to Scott [124]. (Our argument and that of Scott have some parallel features.)

Theorem 9.12. If $G$ is a $\mathrm{PD}_{3}$-group and $K$ is a nontrivial $\mathrm{FP}_{2}$ ascendant subgroup of infinite index in $G$, we are in one of the following situations:

(1) $K$ is a $\mathrm{PD}_{2}$-group, $\left[G: N_{G}(K)\right]$ is finite and $N_{G}(K) / K$ has two ends.

(2) $K \cong \mathbb{Z}$ and $K$ is normal in $G$.

(3) $K \cong \mathbb{Z}$ and $G$ is polycyclic.

Proof. Let $K=K_{0}<K_{1}<\cdots<K_{\beth}=G$ be an ascendant series, and let $\beta=\min \left\{\alpha \mid\left[K_{\alpha}: K\right]=\infty\right\}$. If $K_{\beta}$ is finitely generated then $\beta$ is not a limit ordinal. Hence $\beta=j+1$ for some finite ordinal $j$, and $K_{j}$ is $\mathrm{FP}_{2}$. Hence $K_{j}$ is indecomposable or free, by Theorem 9.7. If $K$ has one end then so does $K_{j}$, and $\left[G: K_{j+1}\right]$ is finite, by Lemma 9.6. Hence $\left[G: N_{G}(K)\right]$ is finite (by the argument in the first paragraph of the proof of Theorem 9.11). Since $N_{G}(K)$ is a $\mathrm{PD}_{3}$-group, $\left[N_{G}(K): K\right]$ is infinite and $K$ has one end, we see that $K$ is a $\mathrm{PD}_{2}$-group and $N_{G}(K) / K$ has two ends, by Theorem 8.9.

If $K$ is free then so is $K_{j}$. Hence $K_{j+1}$ is $\mathrm{FP}_{2}$ and one-ended, by Theorem 9.8, and so is a $\mathrm{PD}_{2}$-group, by the argument just given. But then $K_{j} \cong \mathbb{Z}$ and so $K \cong \mathbb{Z}$.

If $K_{\beta}$ is not finitely generated then it is an open $\mathrm{PD}_{3}$-group, and so $K \cong \mathbb{Z}$, by Theorem 9.11.

In each of these last two cases $K \cong \mathbb{Z}$, and so $K \leqslant \sqrt{G}$, since $K$ is ascendant. Hence either $K$ is normal in $G$ or $G$ is polycyclic, by Theorem 8.10.

The original version of this result [9] assumed that $K$ is subnormal in $G$, and did not use the Bowditch or Algebraic Core Theorems.

The groups considered in Theorem 9.12 are 3-manifold groups $\pi_{1}(M)$, where $M$ is either Seifert fibred or is finitely covered by the total space of a surface bundle over $S^{1}$. If $K$ is a $\mathrm{PD}_{2}$-group, $N_{G}(K)$ is the fundamental group of a 3-manifold which is double covered by the mapping torus of a surface homeomorphism. There are, however, $\mathrm{Nil}^{3}$-groups with no normal $\mathrm{PD}_{2}$-subgroup (although they always have subnormal copies of $\mathbb{Z}^{2}$ ).

Corollary 9.12.1. Let $G$ be a $\mathrm{PD}_{3}$-group and $H=H_{0}$ an $\mathrm{FP}_{2}$ subgroup with one end and of infinite index in $G$. Let $H_{i+1}=N_{G}\left(H_{i}\right)$, for $i \geqslant 0$. Then $\widehat{H}=\bigcup H_{i}$ is $\mathrm{FP}_{2}$ and has one end, and either $\mathrm{cd} \widehat{H}=2$ and $N_{G}(\widehat{H})=\widehat{H}$, or $[G: \widehat{H}]<\infty$ and $G$ is virtually the group of a surface bundle.

Proof. Let $\widehat{H}=\bigcup H_{i}$. Then cd $\widehat{H} \geqslant 2$, since it has a subgroup $H$ with one end. If $[G: \widehat{H}]=\infty$ then cd $\widehat{H}=2$, and so $[\widehat{H}: H]<\infty$, by Lemma 9.10 . Hence $\widehat{H}$ is $\mathrm{FP}_{2}$ and has one end. If $[G: \widehat{H}]<\infty$ then $\widehat{H}$ is again $\mathrm{FP}_{2}$. In each case, $\widehat{H}=H_{i}$ for all $i \gg 0$, and so $N_{G}(\widehat{H})=\widehat{H}$. The final assertion follows from Theorem 9.12.

Corollary 9.12.2. If $G$ has a subgroup $H$ which is a $\mathrm{PD}_{2}$-group with $\chi(H)=0$ (respectively, $\chi(H)<0$ ), then either $G$ has a subgroup I which is also a $\mathrm{PD}_{2}$-group with $\chi(I)=0$ (respectively, $\left.\chi(I)<0\right)$ and which is its own normalizer in $G$, or $G$ is virtually the group of a surface bundle.

Proof. If cd $\widehat{H}=2$ then $[\widehat{H}: H]<\infty$, so $\widehat{H}$ is a $\mathrm{PD}_{2}$-group, and $\chi(H)=[\widehat{H}: H] \chi(\widehat{H})$. 
If $H$ is a subgroup of $G$ which is a $\mathrm{PD}_{2}$-group such that $\chi(H)<0$, then it follows easily from the finite divisibility of $\chi(H)$ that $H$ has finite index in a maximal such subgroup. If $\chi(H)=0$ we may argue instead that an infinite increasing union of copies of $\mathbb{Z}^{2}$ must have cohomological dimension 3 .

The geometric conclusions of Theorem 8.10 and the coherence of 3-manifold groups suggest that Theorems 8.9 and 9.12 should hold under the weaker hypothesis that the ascendant subgroup be finitely generated. (Compare the PD Extension Theorem.)

\subsection{Extending Elkalla's results?}

The argument of Scott in [124] was extended to subnormal subgroups by Elkalla, who showed in [35] that

(1) if $\pi$ is a non-finitely generated 3-manifold group with subgroups $1 \neq N \leqslant U<\pi$ such that $N$ is subnormal in $\pi$ and $U$ is finitely generated then $N \cong \mathbb{Z}$ and is normal in $\pi$; and

(2) if $M$ is a compact $P^{2}$-irreducible 3-manifold and $N \leqslant U$ are such subgroups of $\pi=\pi_{1}(M)$ with $U$ indecomposable and $[G: U]=\infty$, and if $\pi$ is $U$-residually finite, then either $N \cong \mathbb{Z}$ or $M$ is virtually a mapping torus and $U$ is commensurable with $\pi_{1}(F)$, where $F$ is the fibre.

(Here $G$ is $U$-residually finite means that if $g \in G \backslash U$ then there is a subgroup $G_{1}<G$ such that $U \leqslant G_{1}$, $\left[G: G_{1}\right]<\infty$ and $g \notin G_{1}$. In [AFW] the subgroup $U$ is said to be separable in $G$.) When $N=U$ these results follow from Theorems 9.11 and 9.12, since 3-manifold groups are coherent.

If $N$ is normal in $G$ then it is contained in all of the conjugates of $U$, and so $N \leqslant \operatorname{Core}_{G}(U)=$ $\bigcap_{g \in G} g U g^{-1}$. Thus if $U$ is a finitely generated subgroup of a 3-manifold group $\pi$ and $\pi$ is $U$-residually finite, these results imply that either $\operatorname{Core}_{\pi}(U)$ is cyclic or $U$ is commensurable with a subgroup $V$ such that $\left[\pi: N_{\pi}(V)\right]<\infty$, since $\operatorname{Core}_{\pi}(U) \leqslant U$ and is normal in $\pi$.

We cannot expect that $\operatorname{Core}_{G}(U)$ is always finitely generated. Let $M$ be a closed 3-manifold with $H_{1}(M ; \mathbb{Z})$ finite of odd order. Then $\pi=\pi_{1}(M)$ has no quotient with two ends. If $M$ is hyperbolic it is virtually fibred, and $\pi$ is LERF, i.e., is $U$-residually finite for all finitely generated subgroups $U$ [1; AFW, Chapter 5]. Let $K<\pi$ be a subgroup which is a $\mathrm{PD}_{2}$-group such that $\left[\pi: N_{\pi}(K)\right]<\infty$. Let $N=\operatorname{Core}_{\pi}\left(N_{\pi}(K)\right)$ and $L=K \cap N$. Then $N$ is normal in $\pi,[\pi: N]$ is finite and $N / L$ has two ends. After replacing $N$ by the preimage of $\sqrt{N / L}$ and enlarging $L$, if necessary, we may assume that $N / L \cong \mathbb{Z}$. Hence $N^{\prime} \leqslant L$, and so $N^{\prime} \leqslant C L=\operatorname{Core}_{\pi}(L)$. Since $M$ is hyperbolic, $N^{\prime} \neq 1$, so $C L \neq 1$, while $[L: C L]=\infty$, since $\pi / C L$ does not have two ends. Hence $C L$ is not finitely generated.

Can we extend Elkalla's results to our setting? Neither the main result of [35] nor Theorem 9.12 fully encompasses the other.

\subsection{Splitting $\mathbf{P D}_{2}$-subgroups with nontrivial normalizers}

Let $M$ be a $P^{2}$-irreducible 3-manifold. If $F$ is an incompressible closed 2-sided surface in $M$ then either $F \subseteq \partial M$ or $G=\pi_{1}(M)$ splits as an HNN extension $A *_{H} \phi$ or a free product with amalgamation $A *_{H} B$, where $H$ is the image of $\pi_{1}(F)$ in $G$. Moreover, either $M$ is the total space of an $F$-bundle over $S^{1}$, or $F$ bounds a submanifold of $M$ which is the total space of a twisted $I$-bundle over a closed surface, or $H=N_{G}(H)$ [54]. (The cases with $F$ a bounded surface are considered in [55].) In particular, either $H$ is normal in $G$ or $\left[N_{G}(H): H\right] \leqslant 2$. We shall give a short algebraic proof of these facts, with the topological 
hypothesis $M$ has an incompressible embedded surface $F$ replaced by the algebraic consequence $G$ splits over a subgroup $H$.

Theorem 9.13. Let $(G, \Omega)$ be a $\mathrm{PD}_{3}$-pair of groups. If there is a boundary component $H$ such that $N_{G}(H) \neq H$, then $[G: H]=2$ and $(G, \Omega)$ is the peripheral system of a twisted I-bundle.

Proof. The double $D G=D_{\Omega}(G, \Omega)$ is a $\mathrm{PD}_{3}$-group [8, Theorem 8.1]. Since $\left[N_{D G}(H): H\right]$ is clearly infinite, $\left[D G: N_{D G}(H)\right]$ is finite, by Lemma 9.6. Therefore $\left[G: N_{G}(H)\right]$ is also finite. Since $G$ is finitely generated, cd $G=2$ and $H$ is an $\mathrm{FP}_{2}$ subgroup with one end, we see that $[G: H]$ is also finite [Bi, Theorem 8.2]. In particular, $G$ is also a $\mathrm{PD}_{2}$-group. Since $H<N_{G}(H)$, it follows from Lemma 8.6 that $[G: H]=2$, and that $(G, \Omega)$ is the peripheral system of a twisted $I$-bundle.

Corollary 9.13.1. If $G=A *_{H} B$ is a $\mathrm{PD}_{3}$-group which is a nontrivial free product with amalgamation over an $\mathrm{FP}_{2}$ subgroup $H$, and if $\left[G: N_{G}(H)\right]$ is finite, then $H$ is normal in $G$ and $G$ is the group of the union of two twisted I-bundles.

Proof. Since $G$ is a nontrivial free product, $[G: H]$ is infinite. A Mayer-Vietoris argument shows that $H$ cannot be free (compare [Bi, Proposition 9.14]). As $H$ is a normal subgroup of $N_{G}(H)$, which is a $\mathrm{PD}_{3}-$ group, it must be a $\mathrm{PD}_{2}$-subgroup. Therefore $(A, H)$ and $(B, H)$ are $\mathrm{PD}_{3}$-group pairs [8]. Moreover, $H$ is a proper subgroup of each of $N_{A}(H)$ and $N_{B}(H)$, and so $[A: H]=[B: H]=2$, by Theorem 9.13. Hence $H$ is normal in $G$. The final assertion also follows from Theorem 9.13.

Similarly, if $G$ is a nontrivial HNN extension with base $A$ and associated subgroups $H$ and $\phi(H)$, where $H$ is $\mathrm{FP}_{2}$ and has one end, and if $\left[G: N_{G}(H)\right]$ is finite, then $G$ is the group of a surface bundle, and $H$ is normal in $G$ and is the image of the group of a fibre.

In the final corollary we need to assume that the group splits over a surface group. (There is again a similar result for $\mathrm{HNN}$ extensions.)

Corollary 9.13.2. If $G=A *_{H} B$ is a $\mathrm{PD}_{3}$-group which is a nontrivial free product with amalgamation over a $\mathrm{PD}_{2}$-group $H$, and if $\left[G: N_{G}(H)\right]$ is infinite, then $\left[N_{G}(H): H\right] \leqslant 2$.

Proof. The index $\left[N_{G}(H): H\right]$ is finite, by Lemma 9.6. Therefore $\left[N_{A}(H): H\right]$ and $\left[N_{B}(H): H\right]$ are also finite, and at least one is 1 . If $N_{A}(H) \neq H$, say, then $[A: H]=2$ (by Theorem 9.13) and so $N_{G}(A)=A$ and the corollary follows. 



\section{CHAPTER 10}

\section{Splitting along $\mathrm{PD}_{2}$-subgroups}

In $\S 1$ we shall give a brief summary of the key definitions and results of the work of Kropholler and Roller on splitting $\mathrm{PD}_{n}$-groups (with max-c) over subgroups which are $\mathrm{PD}_{n-1}$-groups, and in $\S 2$ we extend some results of theirs on commensurators of $\mathrm{PD}_{2}$-subgroups. Their work led to Kropholler's JSJ decomposition for such groups, which we outline in $\S 3$. There have subsequently been other extensions of the notion of JSJ decomposition, but Kropholler's arguments apply to all $\mathrm{PD}_{3}$-pairs of groups, since they all have max-c. In contrast to the earlier chapters, we shall only give the main definitions and some of the simpler supporting lemmas, and shall not attempt to prove the main results, as the arguments are quite long, and do not involve specifically 3-dimensional aspects. In $\$ 4$ and $\S 5$ we outline in some detail Castel's applications of JSJ decompositions to centralizers and Baumslag-Solitar relations.

\subsection{Kropholler-Roller splitting theorems}

Kropholler and Roller wrote a series of three papers on splitting $\mathrm{PD}_{n}$-pairs of groups along subgroups which are $\mathrm{PD}_{n-1}$-groups. These considered in turn the absolute case [98], the relative case [99], and splitting along virtually solvable groups [101], and ideas from these papers were used also in Kropholler's work on JSJ decompositions of $\mathrm{PD}_{n}$-groups with max-c [94].

Let $G$ a group with a subgroup $H$, and let $\mathcal{P} H$ be the set of all subsets of $H$. A subset $B \subseteq G$ is $H$-finite if $B \subseteq H X$, for some finite $X \subset G$. The set $\mathcal{F}_{H} G$ of $H$-finite subsets of $G$ is an abelian group (of exponent 2) with respect to symmetric difference, and has a natural right $G$-action: $B \mapsto B g$. There is a natural isomorphism

$$
\mathcal{F}_{H} G \cong \mathcal{P} H \otimes_{\mathbb{F}_{2}[H]} \mathbb{F}_{2}[G],
$$

and so $\mathcal{F}_{H} G$ is an induced (right) $\mathbb{F}_{2}[G]$-module.

Let $H$ and $J$ be subgroups which are $\mathrm{PD}_{n-1}$-groups, and let $K=J \cap H$. If $J$ is $H$-finite then $[J: K]<\infty$, and so $K$ is also a $\mathrm{PD}_{n-1}$-group. But then $[H: J]$ is finite also, by the Strebel Theorem, and so $H$ and $J$ are commensurable.

The key definition is based on the following simple lemma.

Lemma 10.1 [99, Lemma 2.1]. Let $(G, \Omega)$ be a $\mathrm{PD}_{n}$-pair of groups and $H$ a subgroup which is a $\mathrm{PD}_{n-1}$ group. Then

$$
H^{1}\left(G, \Omega ; \mathcal{F}_{H} G\right) \cong \mathbb{F}_{2} \text {. }
$$

Proof. Poincaré duality for the pair gives $H^{1}\left(G, \Omega ; \mathcal{F}_{H} G\right) \cong H_{n-1}\left(G ; \mathcal{F}_{H} G\right)$, which, by Shapiro's Lemma and Poincaré duality for $H$, reduces to $H_{n-1}(H ; \mathcal{P} H) \cong H^{0}(H ; \mathcal{P} H) \cong \mathbb{F}_{2}$.

Let $\operatorname{sing}_{G, \Omega}(H)$ be the restriction to $H$ of the nonzero element of $H^{1}\left(G, \Omega ; \mathcal{F}_{H} G\right)$. If $G$ is a $\operatorname{PD}_{n^{-}}$ group we shall write just $\operatorname{sing}(H)$. 
We may now state some of the principal results of [98] and [101]. In each case $G$ is assumed to be a $\mathrm{PD}_{n}$-group.

Theorem [98, Theorem A]. Let $S$ a subgroup which is a $\mathrm{PD}_{n-1}$-group. Then $G$ splits over a subgroup $H$ commensurable with $S$ if and only if $\operatorname{sing}(S)=0$.

If $G$ splits thus, there is exactly one such subgroup $H$.

In [70] it is shown that if a $\mathrm{PD}_{3}^{+}$-group $G$ splits as an $\mathrm{HNN}$ extension $A *_{C} \varphi$ over a $\mathrm{PD}_{2}$-group $C$ then the image of $[C]$ in $H_{2}(G ; \mathbb{Z})$ is Poincaré dual to the epimorphism from $G$ to $\mathbb{Z}$ with kernel $\langle\langle A\rangle\rangle$. This leads to a slight modification of [98]: $G$ splits as an HNN extension over $S$ if and only if $\operatorname{sing}(S)=0$ and $[S]$ generates a $\mathbb{Z}$ direct summand of $H_{2}(G ; \mathbb{Z})$.

Theorem [98, Corollaries A1 and A2]. If $G$ splits over a subgroup $H$ which is a $\mathrm{PD}_{n-1}$-group, then $\left.w_{1}(G)\right|_{H}=w_{1}(H)$, and $H$ is maximal among groups commensurable with $H$ satisfying such a condition on orientation characters. Moreover:

(1) $N_{G}(H)=\operatorname{Comm}_{G}(H)$, and either $\left[N_{G}(H): H\right] \leqslant 2$ or $H$ is normal in $G$.

(2) If $H$ is normal in $G$ then $G / H$ has two ends.

(3) If $\left[N_{G}(H): H\right]=2$ then $G \cong N_{G}(H) *_{H} J$ for some $\mathrm{PD}_{n}$-pair of groups $(J, H)$.

Theorem [98, Theorem B]. Let $S$ be a subgroup which is a $\mathrm{PD}_{n-1}$-group. If there is no $g \in G$ such that $\operatorname{cd}\left(S \cap g S g^{-1}\right)=n-2$, then $G$ splits over a subgroup commensurable with $S$.

A subgroup $L$ is malnormal in $G$ if $L \cap g L g^{-1}=1$ for all $g \notin L$. If $S$ is malnormal in $G$ then $\operatorname{Comm}_{G}(S)=S$, and so if also $n>2$ then $G$ splits over $S$, by the above results. However, $S \times \mathbb{Z}$ splits over $S$, so malnormality is not necessary for splitting.

Lemma [98, Lemma 2.5]. If a subgroup $S$ is a $\mathrm{PD}_{n-1}$-group which is closed in the profinite topology on $G$, then $G$ has a subgroup of finite index which splits over a subgroup commensurable with $S$.

This lemma applies if $G$ is residually finite and $S$ is polycyclic [98, Theorem C]. We shall use this result in Theorem 11.19.

Theorem [101, Theorem A]. If $G$ splits over a virtually solvable subgroup $H$, then $H$ is virtually polycyclic and $h(H)=n-1$.

Theorem [101, Theorem C]. If a subgroup $H$ is isomorphic to a direct product of $n-1$ nontrivial groups, then at most one factor is not cyclic, and any such factor is either free or a $\mathrm{PD}_{2}$-group.

In particular, direct products of two nonabelian free groups cannot be subgroups of $\mathrm{PD}_{3}$-groups. As we observed in Chapter 8, this also follows from the (more recent) Algebraic Core Theorem.

There are analogous results for the relative case in [99]. As these are somewhat more complicated in their formulation, we shall not give them here.

\subsection{Commensurators of $\mathrm{PD}_{2}$-subgroups}

In a fourth joint paper, Kropholler and Roller introduced a variant of the relative end invariant of Houghton and Scott [100]. One of their applications was to show that if $S$ is a $\mathrm{PD}_{2}$-subgroup of a $\mathrm{PD}_{3}$-group $G$ with $\left[\operatorname{Comm}_{G}(S): S\right]=\infty$, then $\left[G: \operatorname{Comm}_{G}(S)\right]<\infty$ and $G$ is virtually a semidirect 
product $S_{0} \rtimes \mathbb{Z}$, for some $S_{0}$ of finite index in $S$ [100, Theorem 1.3 and Proposition 4.4]. We shall give an alternative argument, using results from $\S 4$ and $\S 5$ of Chapter 8.

Theorem 10.2. Let $G$ be a $\mathrm{PD}_{3}$-group with an $\mathrm{FP}_{2}$ subgroup $S$ which has one end. If $\left[\operatorname{Comm}_{G}(S): S\right]=$ $\infty$ then $G$ is virtually a semidirect product $T \rtimes \mathbb{Z}$, where $S$ and $T$ are commensurable.

Proof. We use the notation of [95]. If $X$ is a subset of $G$ let $S^{X}$ be the subgroup generated by the conjugates $S^{x}=x S x^{-1}$, for $x \in X$, and let $S_{X}=\bigcap_{x \in X} S^{x}$. Then $S_{X}$ is normal in $S^{X}$. The union of $S^{X}$ over all finite subsets $X$ of $C=\operatorname{Comm}_{G}(S)$ is $S^{C}=\left\langle\langle S\rangle_{C}=\bigcup S^{X}\right.$.

If $X \subset C$ is finite and $1 \in X$, then $S_{X}$ has finite index in $S$, and so is again $\mathrm{FP}_{2}$ and has one end. Hence if $\left[\operatorname{Comm}_{G}(S): S\right]=\infty$ and $\left[G: S^{X}\right]<\infty$ then we may apply Theorem 8.9: $S_{X}$ is normal in $S^{X}$, and so $S^{X}$ is virtually $S_{X} \rtimes \mathbb{Z}$.

If $\left[G: S^{X}\right]=\infty$ then $\operatorname{cd} S^{X}=2$, and so $\left[S^{X}: S_{X}\right]<\infty$, by [Bi, Corollary 8.6]. Hence $S^{X}$ is again $\mathrm{FP}_{2}$ and has one end. Thus if $\left[G: S^{X}\right]=\infty$ for all finite $X \subset C$ then $\left[S^{C}: S\right]$ is finite, by the GildenhuysStrebel Theorem. Since $S^{C}$ is normal in $C$, either $\left[C: S^{C}\right]$ or $[G: C]$ is finite. Thus either $[C: S]$ is finite, or $C$ is a $\mathrm{PD}_{3}$-group. In the latter case we may again apply Theorem 8.9.

Corollary 10.2.1. If $[G: S]=\infty$ and $S$ is $\mathrm{FP}_{2}$ and has one end, but is not a $\operatorname{PD}_{2}$-group, then $\left[\operatorname{Comm}_{G}(S)\right.$ : $S]<\infty$.

Let $G$ be a torsion-free central extension of a flat 2-orbifold group, such as $S(2,3,6), S(2,4,4)$ or $S(3,3,3)$, by $\mathbb{Z}$. Then $G$ is a polycyclic $\operatorname{PD}_{3}$-group with subgroups $S \cong \mathbb{Z}^{2}$ such that $\operatorname{Comm}_{G}(S)=S$.

Margolis has used coarse geometry to show that if $H$ is an $\mathrm{FP}_{2}$ subgroup of a $\mathrm{PD}_{3}$-group $G$ such that $\operatorname{Comm}_{G}(H)=G$, then $H$ is commensurable with a normal subgroup of $G$ [106].

The next result is a useful complement to Lemma 8.6.

Lemma 10.3 [99, Lemma 2.2]. Let $(G, \Omega)$ be a $\mathrm{PD}_{n}$-pair of groups with nonempty boundary. If $G$ is not of I-bundle type then

(1) $\operatorname{Comm}_{G}(S)=S$, for all boundary components $S$, and

(2) stabilizers of points in distinct $G$-orbits in $\Omega$ are not conjugate to commensurable subgroups.

Proof. Let $T<G$ be a subgroup which is a $\operatorname{PD}_{n-1}$-group. Since $(G, \Omega)$ is not of $I$-bundle type, $G$ is not a $\mathrm{PD}_{n-1}$-group, and so $[G: T]=\infty$. (In particular, $[G: S]=\infty$ for all boundary components $S$.) Then $H^{0}\left(G ; \mathcal{F}_{T} G\right)=0$, since $\mathcal{F}_{T} G$ is induced from an $\mathbb{F}_{2}[T]$-module. Hence

$$
H^{0}\left(\Omega ; \mathcal{F}_{T} G\right)=\oplus_{G_{\eta} \subset \Omega} H^{0}\left(G_{\eta} ; \mathcal{F}_{T} G\right)=\oplus_{G_{\eta} \subset \Omega}\left(\mathcal{F}_{T} G\right)^{G_{\eta}}
$$

is a submodule of $H^{1}\left(G, \Omega ; \mathcal{F}_{T} G\right)$. Since the latter module is $\mathbb{F}_{2}$, at most one of the summands is nonzero. If $T=G_{\tau}$ for some $\tau \in \Omega$ then $T$ is itself a $T$-finite subset which is invariant under right multiplication by $G_{\tau}$, and then the summand corresponding to $\tau$ is nonzero.

If $g \in \operatorname{Comm}_{G}(S)$, where $S$ is a boundary component, then $g S g^{-1}$ is $S$-finite, so $g S$ is $S$-finite. Since it is clearly invariant under right multiplication by $S$, and there is just one such nonempty subset, $g S=S$. Hence $g \in S$, and so $\operatorname{Comm}_{G}(S)=S$.

If $G_{\eta}$ and $g G_{\tau} g^{-1}$ are commensurable, then they are equal, since these groups are their own commensurators, by the preceding paragraph. Since $H^{0}\left(\Omega ; \mathcal{F}_{T} G\right)$ has dimension $\leqslant 1$ this is only possible if $\eta=g \tau$, i.e., they are in the same $G$-orbit. 
In particular, the boundary components corresponding to distinct $G$-orbits in $\Omega$ are pairwise nonconjugate, and are maximal among $\mathrm{PD}_{n-1}$-subgroups. (See [81, Lemma 3.3] for a proof of these assertions based on doubling $G$ over $\mathcal{S}$ and applying the Strebel Theorem.)

The uniqueness results of [98, Corollaries A1 and A2] cited above largely follow from Theorem 10.2 and Lemma 10.3, for $n=3$. For suppose that $G \cong A *_{H} B$ and $\left.\operatorname{Comm}_{G}(H): H\right]<\infty$. If $g \in \operatorname{Comm}_{G}(H)$ then some power of $g$ is in $H$, and so $g$ is in either $A$ or $B$, by a normal form argument. Hence either both of $(A, H)$ and $(B, H)$ are of $I$-bundle type and $H$ is normal in $G$, or just one is of $I$-bundle type and $\left[N_{G}(H): H\right]=2$, or $g \in H$, by Lemma 10.3. A similar argument applies if $G \cong A *_{H} \varphi$.

\subsection{Kropholler's JSJ Decomposition Theorem}

Let $(G, \Omega)$ be a $\mathrm{PD}_{n}$-pair of groups. A reduced $G$-tree $T$ is adapted to $\Omega$ if

(1) each boundary component fixes a vertex of $T$, and

(2) all edge stabilizers are $\mathrm{PD}_{n-1}$-groups.

If $v \in V(T)$, let $\operatorname{Star}(v)=\{e \in E(T) \mid v=o(e)$ or $v=t(e)\}$, and let

$$
\Omega_{v}=\left\{G_{\eta} \mid G_{\eta} \leqslant G_{v}\right\} \sqcup\left\{\iota_{e} \mid e \in \operatorname{Star}(v)\right\},
$$

where $\iota_{e}: G_{e} \rightarrow G_{v}$ is the canonical inclusion. The pair $\left(G_{v}, \Omega_{v}\right)$ is again a $\mathrm{PD}_{n}$-pair of groups, by the Bieri-Eckmann Splitting Theorem [8, Theorem 8.4].

Theorem [94]. Let $(G, \Omega)$ be a $\mathrm{PD}_{n}$-pair of groups with $n \geqslant 3$. If $G$ has max-c but is not virtually polycyclic, then there is a reduced $G$-tree $T$, adapted to $\Omega$, with $G \backslash T$ finite and satisfying these conditions:

(1) $G_{e}$ is virtually polycyclic of Hirsch length $n-1$ for all $e \in E(T)$.

(2) $\left(G_{v}, \Omega_{v}\right)$ is either atoroidal or of Seifert type for all $v \in V(T)$.

(3) Every virtually polycyclic subgroup $H<G$ with $h(H)=n-1$ lies in some vertex stabilizer.

Any two such $G$-trees are $G$-isomorphic.

All $\mathrm{PD}_{3}$-groups and $\mathrm{PD}_{3}$-pairs of groups have max-c, by Theorem 9.5 and the subsequent remarks. Hence we obtain:

Theorem (JSJ Decomposition Theorem [94]). Let $(G, \Omega)$ be a $\mathrm{PD}_{3}$-pair of groups. If $G$ is not polycyclic then there is a reduced $G$-tree $T$, adapted to $\Omega$, with $G \backslash T$ finite and satisfying these conditions:

(1) $G_{e}$ is isomorphic to $\mathbb{Z}^{2}$ or to $\mathrm{Kb}$ for all $e \in E(T)$.

(2) $\left(G_{v}, \Omega_{v}\right)$ is either atoroidal or of Seifert type for all $v \in V(T)$.

(3) Every subgroup $H<G$ which is isomorphic to $\mathbb{Z}^{2}$ or to $\mathrm{Kb}$ lies in some vertex stabilizer.

Any two such $G$-trees are $G$-isomorphic.

At this point, we should observe that the atoroidal vertex groups are never of Seifert type. This follows from the following lemma.

Lemma 10.4. Let $G$ be an extension of a finite group $J$ by a free group $F$ of finite rank $r>1$. Then $G$ has infinitely many conjugacy classes of maximal infinite cyclic subgroups. 
Proof. If $G / G^{\prime}$ has rank at least 2 this is obvious, since conjugate elements of $G$ have the same image in $G / G^{\prime}$, and $G / G^{\prime}$ has infinitely many primitive elements. In general, let $s: J \rightarrow G$ be a section for the epimorphism $G \rightarrow J$. If $w_{1}=g w g^{-1}$ in $G$ and $w^{m} \in F$ then $w_{1}^{m}=f s(j) w^{m} s(j)^{-1} f^{-1}$ in $F$, for some $j \in J$ and $f \in F$. Since there are only finitely many possibilities for $s(j)$ and since $F / F^{\prime} \cong \mathbb{Z}^{r}$, the result follows.

If $(H, \mathcal{T})$ is a $\mathrm{PD}_{3}$-pair of groups of Seifert type and with nonempty boundary, then $\mathrm{cd} H \leqslant 2$, and so $H / \sqrt{H}$ is virtually free. If, moreover, every subgroup $S \cong \mathbb{Z}^{2}$ is conjugate to the image of a member of $\mathcal{T}$ then $H \cong \mathbb{Z}^{2}$ or $H \cong \mathrm{Kb}$, by Lemma 10.4 . Thus $(H, \mathcal{T})$ is of $I$-bundle type, by Lemma 8.6 , and is not atoroidal.

Condition (3) of the JSJ Decomposition Theorem implies that the decomposition is minimal, in that if $e$ is an edge such that $o(e) \neq t(e)$ then $J=\left\langle G_{o(e)}, G_{t(e)}\right\rangle$ is not of Seifert type. For if $\sqrt{J}=\langle j\rangle$ for some $j \neq 1$ then $j \in G_{e}$. Since $T$ is reduced, $G_{e} \neq G_{o(e)}$ and $G_{e} \neq G_{t(e)}$, and we may choose $a \in G_{o(e)}$ and $b \in G_{t(e)}$ such that neither $a$ nor $b$ is in any edge stabilizer, by Lemma 10.3. Since $a$ and $b$ normalize $\langle j\rangle$, the group $\langle a b, j\rangle$ is isomorphic to $\mathbb{Z}^{2}$ to $\mathrm{Kb}$. But this subgroup is not contained in any vertex stabilizer, and so (3) fails. (Similarly, if $o(e)=t(e)$ then $\left\langle G_{o(e)}, t_{e}\right\rangle$ is not of Seifert type.)

We shall refer to the associated graph-of-groups structure for $G$ as the JSJ decomposition of $(G, \Omega)$, and $T$ as the associated Bass-Serre tree. The decomposition is proper if $G \backslash T$ is not a single point. We shall also say that a vertex group $G_{v}$ is atoroidal or of Seifert type if the pair $\left(G_{v}, \Omega_{v}\right)$ is atoroidal or of Seifert type. If a $\mathrm{PD}_{3}$-group $G$ is of Seifert type then it is a Seifert 3-manifold group, by Theorems 8.7 and 8.10. In general, the vertex pairs $\left(G_{v}, \Omega_{v}\right)$ of Seifert type are the peripheral systems of bounded aspherical Seifert fibred 3-manifolds, by Theorem 8.8.

Similar results have since been obtained by Dunwoody and Swenson for $\mathrm{PD}_{3}$-pairs of groups with $G$ finitely presentable [27], and for a much broader class of groups by Scott and Swarup [127; 128].

The main result of [94] applies to $\mathrm{PD}_{n}$-groups with max-c, in all dimensions $n$. Kropholler gave another argument in [97] for the case $n=3$, which did not assume max-c, but had a somewhat weaker conclusion. He showed that if a $\mathrm{PD}_{3}$-group $G$ has an abelian subgroup $A \cong \mathbb{Z}^{2}$ then either $G$ has an infinite cyclic normal subgroup or it is a generalized free product with amalgamation $H *_{K} J$ or an HNN extension $H *_{K} \varphi$, where $K$ is in the class $\mathcal{X}$ (see page 4). The Algebraic Core Theorem implies that noncyclic groups in $\mathcal{X}$ which are subgroups of $\mathrm{PD}_{3}$-groups are the ambient groups of $\mathrm{PD}_{3}$-pairs of groups of Seifert type. See Corollary 10.14.1.

The following result of Dunwoody, showing that $\mathrm{PD}_{n}$-groups do not have unbounded splittings over $\mathrm{PD}_{n-1}$-groups, is used in [94].

Theorem [26]. Let $G$ be a $\mathrm{PD}_{n}$-group and $T$ a reduced $G$-tree in which all edge stabilizers are $\mathrm{PD}_{n-1}{ }^{-}$ groups. Then there is an integer $n(G)$ such that $G \backslash V(T) \leqslant n(G)$ and $G \backslash E(T) \leqslant n(G)$.

\subsection{Castel's Centralizer Theorem}

Castel adapted the JSJ decomposition theory of Scott and Swarup to obtain several striking results on $\mathrm{PD}_{3}$-groups. We shall follow his strategy closely, but shall modify some of the details of the proofs. In particular, we shall cite Kropholler's JSJ Decomposition Theorem rather than the later Scott-Swarup theory. 
We begin with two special cases of Castel's theorem on centralizers which are also ingredients in its proof.

Theorem 10.5. Let $(G, \Omega)$ be a $\mathrm{PD}_{3}$-pair of groups such that $\sqrt{G} \neq 1$ but $G$ is not polycyclic.

(1) If $h \in \sqrt{G}$ then $\left[G: C_{G}(h)\right] \leqslant 2$.

(2) If $h \notin \sqrt{G}$ then $C_{G}(h)$ is isomorphic to $\mathbb{Z}, \mathbb{Z}^{2}$ or $\mathrm{Kb}$.

(3) $\sqrt{G}<S$, for all boundary components $S$.

Proof. Since $G$ is not polycyclic, we have $\sqrt{G} \cong \mathbb{Z}$, and $G / \sqrt{G}$ has no nontrivial finite normal subgroup. (For the preimage of any such subgroup in $G$ would be normal, virtually $\mathbb{Z}$, and torsion-free. Hence it would have to be $\sqrt{G}$.) Moreover, $G / \sqrt{G}$ is isomorphic to a lattice in $\operatorname{PGL}(2, \mathbb{R})$, which is cocompact if $G$ is a $\mathrm{PD}_{3}$-group, and is virtually free otherwise [33].

If $h \in \sqrt{G}$ then $G=N_{G}(\langle h\rangle)$ and $\left[G: C_{G}(h)\right] \leqslant 2$. Elementary matrix calculations show that centralizers of nontrivial elements of $\operatorname{PSL}(2, \mathbb{R})$ are virtually cyclic. Therefore, if $h \notin \sqrt{G}$ then the image of $C_{G}(h)$ in $G / \sqrt{G}$ is virtually cyclic, and so $C_{G}(h)$ is virtually abelian of rank $\leqslant 2$. Hence $C_{G}(h)$ is isomorphic to $\mathbb{Z}, \mathbb{Z}^{2}$ or $\mathrm{Kb}$.

If $\Omega$ is nonempty then $\operatorname{cd} G=2$, and so $\operatorname{cd} \sqrt{G} S \leqslant 2$, for each boundary component $S$. Since $S \cong \mathbb{Z}^{2}$ or $S \cong \mathrm{Kb}$, and $\sqrt{G}$ is normal in $G$, it follows that $S$ has finite index in $\sqrt{G} S$. But $\operatorname{Comm}_{G}(S)=S$, by Lemma 10.3. Therefore $\sqrt{G}<S$.

The next theorem is based on [20, Lemme 3 and Proposition 1], and extends Theorem 9.3 to certain atoroidal $\mathrm{PD}_{3}$-pairs of groups.

Theorem 10.6. Let $(G, \Omega)$ be an atoroidal $\mathrm{PD}_{3}$-pair of groups such that each boundary component $S \cong \mathbb{Z}^{2}$ is malnormal in $G$. If $G$ has a subgroup $H \cong \mathbb{Z} \times F(r)$ then $r \leqslant 1$. Hence if $h \in G \backslash\{1\}$ then $C_{G}(h)$ is a maximal abelian subgroup of $G$.

Proof. We may assume that $(G, \Omega)$ is orientable, and that the boundary is nonempty. If $G$ has such a subgroup with $r>1$, we may assume that $r=2$ and that $H$ has the presentation $\langle a, x, y \mid a \leftrightharpoons x, y\rangle$. Since $(G, \Omega)$ is atoroidal, there are boundary components $S_{i}$ and $g_{i} \in G$, for $1 \leqslant i \leqslant 3$, such that $B=\langle a, x\rangle \leqslant g_{1} S_{1} g_{1}^{-1}, C=\langle a, y\rangle \leqslant g_{2} S_{2} g_{2}^{-1}$ and $D=\langle a, x y\rangle \leqslant g_{3} S_{3} g_{3}^{-1}$.

Let $D_{o o}$ be the "pair of pants" surface. Then $B, C, D<H$ are boundary components for a $\mathrm{PD}_{3}$-pair of groups $(H, \mathcal{T})$ which is the peripheral system of $D_{o o} \times S^{1}$. The inclusion of $H$ into $G$ induces a map of $\mathrm{PD}_{3}$-pairs of groups, from $(H, \mathcal{T})$ to $(G, \Omega)$, and the restrictions to the boundary components $B \mapsto g_{1} S_{1} g_{1}^{-1}, C \mapsto g_{2} S_{2} g_{2}^{-1}$ and $D \mapsto g_{3} S_{3} g_{3}^{-1}$ each have nonzero degree. There is an associated commutative diagram of exact sequences of homology.

If $S_{2}=j S_{1} j^{-1}$ then $g_{1}^{-1} a g_{1} \in S_{1} \cap h S_{1} h^{-1}$, where $h=g_{1}^{-1} g_{2} j$, contrary to the malnormality hypothesis. Therefore no two of the boundary components $S_{i}$ are conjugate in $G$. Consideration of the commutative diagram of homologies shows that $H_{3}(H, \mathcal{T} ; \mathbb{Z})$ maps injectively to $H_{3}(G, \Omega ; \mathbb{Z})$. (See [20, Lemme 3].) Hence the induced map of the doubles $D(H, \mathcal{T}) \rightarrow D(G, \Omega)$ has nonzero degree, and so the image has finite index, by Lemma 8.5. Therefore $[G: H]$ is finite, and so $H$ is atoroidal. But then $r \leqslant 1$, by Lemma 10.4 .

If $h \in G \backslash\{1\}$ and $C=C_{G}(h)$ is nonabelian then $\operatorname{cd} C=\operatorname{cd} G=2$, since $C$ is not free, and $C^{\prime}$ is a free group such that $C^{\prime} \cap\langle h\rangle=1$. Hence $C$ has a subgroup $H \cong \mathbb{Z}^{2}$, which contains $h$. This is conjugate into 
one of the boundary components $S$, since $(G, \Omega)$ is atoroidal. Clearly $S \cong \mathbb{Z}^{2}$, and so $S$ is malnormal in $G$. We may assume that $H \leqslant S$. Then $C \leqslant S$ also, since $S$ is malnormal in $G$, and so $C$ is abelian after all. It is clearly maximal among such subgroups.

In order to prove the main theorem, which extends Theorems 10.5 and 10.6 to the cases with proper JSJ decompositions, we shall need to show that if $(G, \Omega)$ is an atoroidal $\mathrm{PD}_{3}$-pair of groups and a boundary component $S$ is abelian then $S$ is malnormal in $G$. As we have not understood some details of the argument for this in [20, Lemme 2], we shall cite a parallel result of Scott and Swarup, which is a consequence of their work on JSJ decompositions. (We have paraphrased their result.)

Theorem [128, Proposition 5.3]. Let $(G, \Omega)$ be an atoroidal orientable $\mathrm{PD}_{n}$-pair of groups which is not of I-bundle type. Suppose that $\eta, \omega \in \Omega$ are such that $G_{\eta}$ and $G_{\omega}$ are virtually polycyclic, and that there are subgroups $S<G_{\eta}$ and $T<G_{\omega}$ of Hirsch length $n-2$ and an element $g \in G$ such that $S=g T g^{-1}$. Then $\omega=\eta$ and $g \in G_{\eta}$.

When $n=3$ the malnormality condition follows from Lemma 10.3 and this result.

Lemma 10.7 [20, Lemme 2]. Let $(G, \Omega)$ be an atoroidal orientable $\mathrm{PD}_{3}$-pair of groups. If $G_{\omega} \cong \mathbb{Z}^{2}$ for some $\omega \in \Omega$, then $G_{\omega}$ is malnormal in $G$.

Proof. Suppose that $g \in G$, and let $S=g G_{\omega} g^{-1} \cap G_{\omega}$ and $T=G_{\omega} \cap g^{-1} G_{\omega} g$. Then $S=g T g^{-1}$. If $g \notin G_{\omega}$ then $G_{\omega}$ and $g G_{\omega} g^{-1}$ are not commensurable, by Lemma 10.3, and so $\operatorname{cd} S=\operatorname{cd} T \leqslant 1$. We cannot have $S \cong T \cong \mathbb{Z}$, by the result just cited, and so $g G_{\omega} g^{-1} \cap G_{\omega}=1$.

Lemma 10.8 [DD, Proposition I.4.7]. Let $G$ be a group which acts on a tree T. Then $G$ stabilizes a vertex of $T$ if and only if there is a vertex $v$ and an integer $N$ such that $d(v, g \cdot v) \leqslant N$ for all $g \in G$.

Proof. The condition is clearly necessary. Suppose that it holds, and let $U$ be the subtree with vertex set the orbit $G \cdot v$. Then $U$ is $G$-invariant, and has diameter $\leqslant 2 N$. The extreme distance is realized by pairs of vertices of valence 1 . Since this subset is invariant under $G$, discarding the extreme vertices gives a $G$-tree of strictly smaller diameter. The lemma follows by induction on the diameter.

Lemma 10.9 [20, Lemme 4]. Let $(G, \Omega)$ be a $\mathrm{PD}_{3}$-pair of groups with a proper JSJ decomposition. Let $\gamma$ be a path in the Bass-Serre tree T associated to the JSJ decomposition. If there is a nontrivial element $h \in G$ which fixes $\gamma$ pointwise, then $\gamma$ has length $\leqslant 2$.

Proof. We note first that if $v$ is a vertex of $\gamma$ which is not an endpoint, then $G_{v}$ must be of Seifert type. For if $G_{v}$ is atoroidal and $e, f$ are edges of $\gamma$ meeting at $v$, then the edge groups $G_{e}, G_{f}$ both contain $h$. Since $h$ is central in $\left\langle G_{e}, G_{f}\right\rangle$ and $G_{v}$ is not of Seifert type, $\left\langle G_{e}, G_{f}\right\rangle /\langle h\rangle$ is virtually free, and so $\left\langle G_{e}, G_{f}\right\rangle$ must be virtually $\mathbb{Z}^{2}$, by Theorem 10.6. But this contradicts Lemma 10.3.

If $\gamma$ has length $>2$ then there are a pair of adjacent interior vertices, $w$ and $z$, say, connected by an edge $e$. Since $h$ fixes each of the edges ending at $w$, it is in $\sqrt{G_{w}}$. (For otherwise the corresponding edge groups would be commensurable, since they each meet $\sqrt{G_{w}}$ nontrivially. But this would again contradict [98, Corollary A1].) A similar argument applies for the vertex $z$, and so $h \in \sqrt{G_{w}} \cap \sqrt{G_{z}}$. Thus $\langle h\rangle$ is normal in $H=\left\langle G_{w}, G_{z}\right\rangle=G_{w} *_{G_{e}} G_{z}$, and so $\sqrt{H} \neq 1$. Let $g_{w} \in G_{w}$ and $g_{z} \in G_{z}$ be elements which are not in $\sqrt{H}$. Then $k=\left(g_{w} g_{z}\right)^{2}$ commutes with $h$, but $\langle h, k\rangle$ is not conjugate into any vertex group of the JSJ decomposition. This contradicts property (3) of the decomposition. Thus $\gamma$ has at most one internal vertex, and so has length $\leqslant 2$. 
Lemma 10.10 [20, Lemme 5]. Let $(G, \Omega)$ be a $\mathrm{PD}_{3}$-pair of groups which has a proper JSJ decomposition. If $h \in G \backslash\{1\}$ fixes a vertex $v$ of the the Bass-Serre tree $T$ associated to the JSJ decomposition of $G$, then $C_{G}(h)$ fixes a vertex of $T$.

Proof. Let $a \in C_{G}(h)$. If $A=\langle a, h\rangle \cong \mathbb{Z}^{2}$ then $A$ fixes a vertex $v^{\prime}$ of $T$, by condition (3) of the JSJ Decomposition Theorem. If $A \cong \mathbb{Z}=\langle t\rangle$, say, then $a=t^{m}$ and $h=t^{n}$ for some exponents $m, n$. Since $t^{n}$ fixes $v, t$ must fix a vertex $v^{\prime}$, say. Hence $h$ fixes $v$ and $v^{\prime}$ also. In each case, $d\left(v, v^{\prime}\right) \leqslant 2$, by Lemma 10.9. Hence

$$
d(v, a \cdot v) \leqslant d\left(v, v^{\prime}\right)+d\left(v^{\prime}, a \cdot v\right)=d\left(v, v^{\prime}\right)+d\left(a \cdot v^{\prime}, a, v\right) \leqslant 4 .
$$

Since this estimate holds for each $a \in C_{G}(h)$, it follows that $C_{G}(h)$ fixes a vertex of $T$.

The next theorem is the main result of this section.

Theorem 10.11 (Castel's Centralizer Theorem [20, Théorème 1]). Let $(G, \Omega)$ be a $\mathrm{PD}_{3}$-pair of groups. If $h \in G$ is not infinitely divisible, we are in one of these situations:

(1) $C_{G}(h)$ is isomorphic to $\mathbb{Z}$.

(2) $C_{G}(h)$ is isomorphic to $\mathbb{Z}^{2}$ or $\mathrm{Kb}$.

(3) $C_{G}(h)$ is conjugate to a subgroup of index at most 2 in one of the Seifert pieces of the JSJ decomposition of $G$.

If $h$ is infinitely divisible and $h \neq 1$, then $C_{G}(h)$ fixes an atoroidal vertex of the JSJ decomposition of $G$, and is abelian of rank 1 , but not finitely generated.

Proof. We may assume that $G$ is not polycyclic. We may also assume that $G$ is orientable. (Note that a torsion-free group which is virtually abelian of rank 1 must be abelian.)

Assume first that $h$ is not infinitely divisible, and let $C=C_{G}(h)$. If cd $C=1$ then $C \cong \mathbb{Z}$, while if $\operatorname{cd} C=3$ then $\sqrt{G} \neq 1$, and (3) holds. Hence we may assume that $\operatorname{cd} C=2$, and that $C$ is not virtually $\mathbb{Z}^{2}$. Therefore $C^{\prime}$ is a nonabelian free group and $\zeta C \cong \mathbb{Z}$. We may assume without loss of generality that $h$ generates $\zeta C$. As in Theorem 10.6, $C$ has a subgroup $H \cong \mathbb{Z}^{2}$, which contains $h$. This must fix a vertex of the Bass-Serre tree $T$ associated to the JSJ decomposition, by part (3) of the JSJ Decomposition Theorem. But then $C$ also fixes a vertex $v \in V(T)$, by Lemma 10.10. Since $C$ has nontrivial centre, $G_{v}$ is of Seifert type, and (3) then follows from Theorem 10.5.

Assume now that $h \neq 1$ is infinitely divisible. If $h$ does not fix a vertex of $T$ then there is an unique axis which is invariant under $h$, and along which $h$ has a well-defined, integral translation length $\ell(h) \neq 0$ [Se, Proposition 24]. If $h=g^{k}$ for some $g \in G$ then $g$ is also fixed-point free, and $\ell(h)=k \ell(g)$. Hence $|k| \leqslant \ell(g)$, contrary to hypothesis. Therefore $h$ fixes a vertex of $T$. It follows from Lemma 10.10 that $C_{G}(h)$ also fixes a vertex $v \in V(T)$. Since groups of Seifert type do not have infinitely divisible elements, $G_{v}$ must be atoroidal. Hence $C_{G}(h)$ is abelian, by Theorem 10.6. It is not finitely generated, since $h$ is infinitely divisible. Therefore $\operatorname{cd}_{G}(h) \leqslant 2$, and so $C_{G}(h)$ has rank 1 .

With Theorem 9.5, it follows that if every abelian subgroup is finitely generated then all centralizers are finitely generated, as asserted at the end of $\S 1$ of Chapter 9.

It is a consequence of the Geometrization Theorem that 3-manifold groups have no infinitely divisible elements. However, this remains an open question for $\mathrm{PD}_{3}$-groups in general. 


\subsection{No nontrivial Baumslag-Solitar relations}

If $G$ is a torsion-free group then we shall say that $x, y \in G$ satisfy a nontrivial Baumslag-Solitar relation if $x, y \neq 1$ and $x y^{p} x^{-1}=y^{q}$ for some exponents $p, q \neq 0$ such that $|p| \neq|q|$. Castel showed that no nontrivial Baumslag-Solitar relation holds in any $\mathrm{PD}_{3}$-group. The argument involves reduction to the following special case.

Theorem 10.12 [81]. Let $G$ be a $\mathrm{PD}_{3}$-group. If the Baumslag-Solitar group $\mathrm{BS}(1, q)$ is a subgroup of $G$, then $q= \pm 1$.

Proof. The Baumslag-Solitar groups BS $(p, q)$ are finitely presentable and have one end. Therefore if $H=\mathrm{BS}(1, q)<G$ then $H$ is the ambient group of a $\mathrm{PD}_{3}$-pair $(H, \mathcal{T})$, by the Algebraic Core Theorem. On passing to a subgroup of index 2 , if necessary, we may assume that $(H, \mathcal{T})$ is orientable. Since $H_{1}(H ; \mathbb{Z})=\mathbb{Z} \oplus(\mathbb{Z} /(q-1) \mathbb{Z})$ and $H_{i}(H ; \mathbb{Z})=0$ for $i>1$, it follows from the exact sequence of the pair that there are 1 or 2 boundary components, each with group $\mathbb{Z}^{2}$. But $\mathbb{Z}^{2}$ is only a subgroup of $\operatorname{BS}(1, q)$ if $q= \pm 1$.

We shall use the following lemma, rather than appealing to the topology of Seifert fibred 3-manifolds. Lemma 10.13. Let $(G, \Omega)$ be a $\mathrm{PD}_{3}$-pair of groups of Seifert type. Then no nontrivial Baumslag-Solitar relation holds in $G$.

Proof. We may assume that $G$ is not polycyclic. Then $\sqrt{G} \cong \mathbb{Z}$ and $G / \sqrt{G}$ has a subgroup $K$ of finite index $m$ which is either a $\mathrm{PD}_{2}$-group (if $\mathcal{S}$ is empty) or a free group. We may also assume that $\sqrt{G}$ is central in the preimage of $K$ in $G$. Suppose that $x, y \in G$ satisfy $x^{p}=y x^{q} y^{-1}$, and let $r=p^{m}$ and $s=q^{m}$. Then $u=x^{m}$ and $v=y^{m}$ satisfy $u^{r}=v u^{s} v^{-1}$, and the images of $u$ and $v$ in $G / \sqrt{G}$ are in $K$. No nontrivial Baumslag Solitar relation holds in any $\mathrm{PD}_{2}$-group or free group, so either $r= \pm s$, in which case $p= \pm q$, or $u \in \sqrt{G}$. But in the latter case $u^{r}=v u^{s} v^{-1}=u^{s}$, so either $r=s$ and $p=q$, or $u=1$, in which case $x=1$.

We may now give Castel's proof of his theorem.

Theorem 10.14 [20, Théorème 2]. Let $(G, \Omega)$ be a $\mathrm{PD}_{3}$-pair of groups. Then no nontrivial BaumslagSolitar relation holds in $G$.

Proof. It is sufficient to prove this in the absolute case, for if a nontrivial Baumslag-Solitar relation holds in $G$ then it holds also in the $\mathrm{PD}_{3}$-group obtained by doubling $(G, \Omega)$ along its boundary.

Suppose then that $G$ is a $\mathrm{PD}_{3}$-group and $x y^{p} x^{-1}=y^{q}$ for some $x, y \in G \backslash\{1\}$. Let $H$ be the subgroup generated by $x$ and $y$. It is clear that $x, y \in \operatorname{Comm}_{H}(\langle y\rangle)$, and so $\operatorname{Comm}_{H}(\langle y\rangle)=H$. Therefore, there are functions $p, q: H \rightarrow \mathbb{Z} \backslash\{0\}$ such that

$$
g y^{p(g)} g^{-1}=y^{q(g)}, \quad \text { for all } g \in H .
$$

Following [96], we may define a homomorphism $\Psi: H \rightarrow \mathbb{Q}_{+}^{\times}$by

$$
\Psi(g)=\left|\frac{p(g)}{q(g)}\right|, \quad \text { for all } g \in H .
$$

Clearly $\Psi(x)=\left|\frac{p}{q}\right| \neq 1$, while $\Psi(y)=1$, and $\operatorname{so} \operatorname{Im}(\Psi) \cong \mathbb{Z}$. 
Let $T$ be the $G$-tree associated to the JSJ decomposition of $G$. If $y$ fixes no vertex of $T$ then it has an invariant axis, on which $y$ acts by translation, with translation length $\ell(y)=d(a, y \cdot a)>0$, for all $a \in A$ [Se, Proposition 24]. The translation length is additive and invariant under conjugation, which quickly leads to a contradiction, since $\ell\left(y^{p}\right)=\ell\left(x y^{p} x^{-1}\right)=\ell\left(y^{q}\right)$. Therefore $y$ fixes some $v \in V(T)$. For each $g \in H$ we have

$$
d\left(g^{-1} \cdot v, y^{p(g)} g^{-1} \cdot v\right)=d\left(v, g y^{p(g)} g^{-1} \cdot v\right)=d\left(v, y^{q(g)} \cdot v\right)=0 .
$$

Since $y^{p(g)} \neq 1$ fixes both $v$ and $g^{-1} \cdot v$, it follows from Lemma 10.9 that $d\left(v, g^{-1} \cdot v\right) \leqslant 2$. Thus $H \cdot v$ lies in a bounded neighbourhood of $v$ and so is finite. Therefore $H$ fixes a vertex $w \in V(T)$.

Since no nontrivial Baumslag-Solitar relation holds in any group of Seifert type, by Lemma 10.13, $G_{w}$ is atoroidal. Let $g \in \operatorname{Ker}(\Psi)$. Then $g^{2} y^{p(g)} g^{-2}=y^{p(g)}$, and so $y^{p(g)} \in C_{G_{w}}\left(g^{2}\right)$. Since $G_{w}$ is atoroidal this centralizer is abelian, and so $g$ commutes with $y^{p(g)}$. If $h$ is another element of $\operatorname{Ker}(\Psi)$ then $g, h$ are both in $C_{G_{w}}\left(y^{p(g) p(h)}\right)$, which is again abelian. Hence $\operatorname{Ker}(\Psi)$ is abelian.

Since $\operatorname{Im}(\Psi) \cong \mathbb{Z}$ and $\operatorname{Ker}(\Psi)$ is abelian, $H$ is solvable. If $[G: H]$ is finite then $H$ is polycyclic of Hirsch length 3 , and it is easy to see that no nontrivial Baumslag-Solitar relation holds in any such group. Therefore $\mathrm{cd} H=2$, and so $H$ must be an ascending $\mathrm{HNN}$ extension $\mathrm{BS}(1, m)=\mathbb{Z} *_{m}$, for some $m \neq 0$. But then $m= \pm 1$, by Theorem 10.12 .

Corollary 10.14.1. Let $(G, \Omega)$ be a $\mathrm{PD}_{3}$-pair of groups. Let $H$ be a subgroup of $G$ which is in the class $\mathcal{X}$. Then $\sqrt{H} \neq 1$, and either $H \cong \mathbb{Z}$ or $H$ is the ambient group of a $\mathrm{PD}_{3}$-pair of groups of Seifert type. In the latter case, $\sqrt{H} \cong \mathbb{Z}$ or $\sqrt{H} \cong \mathbb{Z}^{2}$.

Proof. We may assume that $H \not \mathbb{Z}$, and so cd $H=2$. As $H$ is $\mathrm{FP}_{2}$ and has one end, it is the ambient group of a $\mathrm{PD}_{3}$-pair of groups $(H, \mathcal{T})$, by the Algebraic Core Theorem. We also have $H \cong \pi \mathcal{G}$, where $(\mathcal{G}, \Gamma)$ is a finite graph of groups in which all vertex and edge groups are infinite cyclic. Since no nontrivial Baumslag-Solitar relations hold in $G$, we must have $\left[G_{o(e)}: G_{e}\right]=\left[G_{t(e)}: G_{e}\right]$ for all edges $e \in E(\Gamma)$. It follows easily that $\sqrt{H} \neq 1$. Hence $(H, \mathcal{T})$ is of Seifert type. The final assertion is clear.

As an easy consequence of his two main results, Castel gave a parallel result for commensurators.

Theorem 10.15 [20, Proposition 4]. Let $(G, \Omega)$ be a $\mathrm{PD}_{3}$-pair of groups. If $h \in G$ is not infinitely divisible, we are in one of these situations:

(1) $\operatorname{Comm}_{G}(\langle h\rangle)$ is isomorphic to $\mathbb{Z}$.

(2) $\operatorname{Comm}_{G}(\langle h\rangle)$ is isomorphic to $\mathbb{Z}^{2}$ or $\mathrm{Kb}$.

(3) $\operatorname{Comm}_{G}(\langle h\rangle)$ is isomorphic to the fundamental group of a Seifert fibred 3-manifold.

If $h$ is infinitely divisible and $h \neq 1$, then $\operatorname{Comm}_{G}(\langle h\rangle)$ is isomorphic to a non-cyclic subgroup of $\mathbb{Q}$.

Proof. Let $x \in \operatorname{Comm}_{G}(\langle h\rangle)$. Then there are exponents $p(x)$ and $q(x)$ such that $x h^{p(x)} x^{-1}=h^{q(x)}$, by the definition of the commensurator. Since $|p(x)|=|q(x)|$, by Theorem $10.14, x^{2}$ commutes with $h^{p(x)}$. The chain of centralizers $C_{G}\left(h^{n !}\right)$ is finite, by Theorem 9.5, and so there is an exponent $p$ such that $x^{2} \in C_{G}\left(h^{p}\right)$ for all $x \in \operatorname{Comm}_{G}(\langle h\rangle)$. The result now follows from Theorem 10.11.

The argument shows that $\operatorname{Comm}_{G}(\langle h\rangle)=N_{G}\left(\left\langle h^{n !}\right\rangle\right)$ for $n$ large. 


\section{CHAPTER 11}

\section{The Tits Alternative}

A group satisfies the Tits Alternative if every finitely generated subgroup is either virtually solvable or has a nonabelian free subgroup. This expression derives from a well-known result of Tits, who showed that linear groups (in characteristic 0) satisfy this alternative. Thus it is a necessary condition for the group to have a faithful linear representation.

Interestingly, much of the work towards showing that $\mathrm{PD}_{3}$-groups might satisfy the Tits Alternative makes use of profinite or pro- $p$ completions, or related ideas like $p$-adic analytic groups [89; 90; 109; 115]. (This is implicit also in [14].)

We begin with a relatively simple argument (from [63]) for the Tits Alternative for almost coherent subgroups of infinite index. We then recall the definition of profinite and pro- $p$ completions, and show that the derived series and lower central series of $\mathrm{PD}_{3}$-groups either terminate early or continue indefinitely. In Sections 4 and 5 (which are based on [14]) we show that the Tits Alternative holds for almost coherent $\mathrm{PD}_{3}$-groups which are not virtually "properly locally cyclic". In the final section we consider "largeness".

\subsection{The virtually Haken case}

Most 3-manifold groups are linear over $\mathbb{Z}$ [AFW, Flowchart 4]. (This is not known for the fundamental groups of proper graph manifolds admitting no non-positively curved metric.) All 3-manifold groups satisfy the Tits Alternative. This is easy to see for Seifert fibred 3-manifolds and hyperbolic 3-manifolds, and was proven for virtually Haken 3-manifolds in [37]. These three classes include all aspherical closed 3-manifolds, by the Geometrization Theorem.

Theorem 11.1. Let $H$ be an $\mathrm{FP}_{2}$ group and $\phi: H \rightarrow H$ an injective endomorphism. If the HNN extension $G=H *_{\phi}$ is a $\mathrm{PD}_{3}$-group then $\phi$ is an isomorphism. and hence $H$ is normal in $G$.

Proof. If $G$ is a $\mathrm{PD}_{3}$-group then $\mathrm{cd} H=2$, by the Strebel Theorem. Let $X$ be the tree associated to the HNN extension. Let $\mathcal{D}^{2}$ be the local coefficient system on $X$ which associates to each vertex or edge the group $H^{2}(H ; \mathbb{Z}[H])$, and to the inclusions of the initial and terminal vertices of an edge the identity homomorphism and the restriction induced by $\phi$, respectively. Then $H^{3}(G ; \mathbb{Z}[G]) \cong H_{c}^{1}\left(X ; \mathcal{D}^{2}\right)$ [16, Theorem 3.2]. This is in turn a direct $\operatorname{limit} \underset{\lim }{\longrightarrow} M_{k}$, indexed by $\mathbb{Z}$, where the map from $M_{k}$ to $M_{k+1}$ is equivalent to the direct sum of $[H: \phi(H)]$ copies of the restriction map from $H^{2}(H ; \mathbb{Z}[H])$ to $H^{2}(\phi(H) ; \mathbb{Z}[H])[16$, Remark 3.5]. Thus this direct limit can only be infinite cyclic if $[H: \phi(H)]=1$.

In [70] the Algebraic Core Theorem and [16] are used to show that no $\mathrm{FP}_{2}$ subgroup of a $\mathrm{PD}_{3}$-group is a properly ascending HNN extension. (See also Theorems 11.1 and 11.2.) 
A group $G$ is restrained if it has no nonabelian free subgroup.

Theorem 11.2. Let $G$ be a $\mathrm{PD}_{3}$-group and $H$ an almost coherent restrained subgroup of infinite index. Then either $H \cong \mathbb{Z}^{2}$ or $H \cong \mathrm{Kb}$ or $H$ is abelian of rank 1 .

Proof. We may assume that $H$ is not abelian of rank 1. Suppose first that $H$ is finitely generated, and hence $\mathrm{FP}_{2}$ (since it is almost coherent). Since $H$ is restrained and not $\mathbb{Z}$, it has one end, and c.d.H=2. Hence $H$ is the ambient group of a $\mathrm{PD}_{3}$-pair of groups $(H, \mathcal{S})$, by the Algebraic Core Theorem. Moreover, $\chi(\operatorname{Im}(\sigma))=0$ for all $\sigma \in \mathcal{S}$, since $H$ is restrained, and so $\chi(H)=0$, by Lemma 3.1. Hence $H^{1}(H ; \mathbb{Z}) \neq 0$, and so $H$ is an HNN extension with finitely generated base, by the Bieri-Strebel Theorem. The extension must be ascending, since $H$ is restrained, and the base $B$ is again $\mathrm{FP}_{2}$, since $H$ is almost coherent. Now $H^{2}(H ; \mathbb{Z}[H]) \neq 0$, since $c . d . H=2$, and so $H^{1}(B ; \mathbb{Z}[H]) \neq 0[16$, Theorem 0.1$]$. Hence $B$ has one end, and so $H \cong \mathrm{BS}(1, m)$, for some $m \neq 0$. In fact, we must have $m= \pm 1$, and so $H \cong \mathbb{Z}^{2}$ or $H \cong \mathrm{Kb}$, by Theorem 10.12.

In general, $H$ is an increasing union of such subgroups, and so is virtually abelian of rank 2 . Since cd $H=2$, by the Strebel Theorem, $H$ must be finitely generated.

Corollary 11.2.1. Let $G$ be a $\mathrm{PD}_{3}$-group. Then $G$ is polycyclic if and only if it is coherent, it is restrained and either $v \beta(G)>0$ or $G$ is virtually split over a finitely generated subgroup.

Proof. The conditions are clearly necessary. For the converse, we may assume that $G$ is orientable and either $G / G^{\prime}$ is infinite or $G \cong A *_{C} B$, where $C$ is a $\mathrm{PD}_{2}$-group. In the first case, $G$ is an $\mathrm{HNN}$ extension with finitely generated base $B$ and associated subgroups, by the Bieri-Strebel Theorem. The HNN extension is ascending, since $G$ is restrained, and $B$ is $\mathrm{FP}_{2}$, since $G$ is almost coherent. Hence $B$ is a restrained $\mathrm{PD}_{2}$-group, and $G \cong B \rtimes \mathbb{Z}$, by Theorems 8.9 and 11.1 . Thus $G$ is polycyclic. In the second case, $A, B$ and $C$ are each finitely generated [Bi, Proposition 2.13] and of infinite index, and $\operatorname{cd} A=\operatorname{cd} B=\operatorname{cd} C=2$. Hence $A, B$ and $C$ are virtually abelian of rank 2 , by Theorem 11.2 , and so $[A: C]$ and $[B: C]$ are finite. Therefore $[A: C]=[B: C]=2$, by Lemma 8.6, and so $C$ is a normal subgroup of $G$ with quotient $G / C \cong D_{\infty}$.

Theorem 11.2 and Corollary 11.2.1 show that the fundamental groups of virtually Haken 3-manifolds satisfy the Tits Alternative, since such groups are coherent $\mathrm{PD}_{3}$-groups.

In any group the subgroup generated by normal restrained subgroups is again normal and restrained, and so there is an unique maximal normal restrained subgroup.

Corollary 11.2.2. Let $G$ be an almost coherent $\mathrm{PD}_{3}$-group, and let $N$ be its maximal normal restrained subgroup. If $N \neq G$ then $N \cong \mathbb{Z}$ or $N=1$.

Proof. Since $N \neq G$, it has infinite index in $G$. Moreover, $\sqrt{G}$ is cyclic, by Theorem 8.9 , since $G$ is not itself restrained. If $N$ is not abelian then $N \cong \mathrm{Kb}$, by Theorem 11.2. But then $h(\sqrt{N})=2$ and so $h(\sqrt{G}) \geqslant 2$, contradicting Theorem 8.9. If $N$ is abelian then $N \leqslant \sqrt{G}$, and so $N \cong \mathbb{Z}$ or $N=1$.

We expect that if $N=G$ then $G$ is polycyclic. The maximal elementary amenable normal subgroup $E$ is virtually solvable [73]. If $E \neq 1$ then $\sqrt{G} \neq 1$, and so either $E$ is cyclic or $G$ is polycyclic. by Theorem 8.10.

A group $G$ is indicable if there is an epimorphism from $G$ to $\mathbb{Z}$, and is locally indicable if all of its finitely generated subgroups are indicable. 
Theorem 11.3. Let $G$ be an almost coherent $\mathrm{PD}_{3}$-group such that $G / G^{\prime}$ is infinite. Then $G$ is locally indicable.

Proof. Let $H$ be a finitely generated subgroup of $G$. If $H$ has finite index in $G$ then its image in $G / G^{\prime}$ is infinite. If $[G: H]=\infty$ then the indecomposable factors of $H$ are either $\mathbb{Z}$ or are ambient groups of $\mathrm{PD}_{3}$-pairs of groups, by the Algebraic Core Theorem. In all cases $H$ is indicable.

Can this result be strengthened to show that every $\mathrm{PD}_{3}$-group is virtually residually solvable?

\subsection{Profinite and pro- $p$ completions}

The set of all normal subgroups $N$ of finite index in a (finitely generated) group $G$ forms a directed system $\mathfrak{N}$, and $G$ is residually finite if $\bigcap \mathfrak{N}=1$. The profinite completion of $G$ is

$$
\widehat{G}=\lim _{N \in \mathfrak{N}} G / N .
$$

There is a natural homomorphism from $G$ to $\widehat{G}$, which is a monomorphism if and only if $G$ is residually finite. Since the projective limit is a subgroup of the direct product $\prod_{N \in \mathfrak{N}} N$ defined by equations, $\widehat{G}$ has a natural (metrizable) topology, as a compact totally disconnected topological group. The kernels of the projections of $\widehat{G}$ onto $G / N$, for $N \in \mathfrak{N}$, form a basis of open neighbourhoods of the identity. The image of $G$ in $\widehat{G}$ under the natural homomorphism is dense. If $p$ is a prime, the pro-p completion $\widehat{G}_{p}$ is defined similarly, using the set of all normal subgroups of index a power of $p$. Clearly, if $\widehat{G}_{p}$ is infinite then so is $\widehat{G}$.

Pro- $p$ completions were used in [29] to study $\mathrm{PD}_{2}$-groups, and the profinite topology was used in connection with the relative end invariant by Scott [125]. The latter use played a part in the proof of [98, Theorem C], which is used in Theorem 11.19 below. The related notion of $p$-adic analytic group underlies part of the arguments of [109].

The cohomological properties of pro- $p$ groups are in some ways better understood than those of general profinite groups, but requiring that $\widehat{G}_{p}$ be infinite is a more restrictive condition. We refer to [90; 156] for the notion of pro- $p$ Poincare duality group. In [90] it is shown that if $G$ is a $\mathrm{PD}_{3}$-group such that $\widehat{G}_{p}$ is infinite, then either $\widehat{G}_{p} \cong \widehat{\mathbb{Z}}_{p}$, or $\widehat{G}_{p}$ is a pro- $p$ Poincaré duality group and every subgroup of finite index in $\widehat{G}_{p}$ has deficiency 0 , or $\widehat{G}_{p}$ is not a pro- $p$ Poincaré duality group and $\widehat{G}_{p}$ has subgroups of finite index with arbitrarily large deficiency. (This includes the result of [156] that if $\widehat{G}_{p}$ is infinite, and if every subgroup of finite index in $G$ has finite abelianization, then $\widehat{G}_{p}$ is a pro- $p$ Poincaré duality group.)

If $G$ is residually finite and $\left\{N_{k}\right\}$ is a descending sequence of normal subgroups of finite index such that $\bigcap N_{k}=1$, then

$$
\limsup \frac{\beta_{1}\left(N_{k}\right)}{\left[G: N_{k}\right]}=\beta_{1}^{(2)}(G)
$$

by the $L^{2}$-Approximation Theorem [Lu, page 453]. (It is easy to see that if $G$ is any quotient of $F(r)$ and $N$ is any subgroup, then $\beta_{1}(N) /[G: N] \leqslant r$.) If $\pi$ is a 3-manifold group then it is residually finite [AFW], and $\beta_{1}^{(2)}(\pi)=0[\mathrm{Lu}$, Theorem 4.1]. (Each of these results relies on the Geometrization Theorem.) 


\subsection{Nilpotent and solvable quotients}

The derived series $G^{(n)}$ and lower central series $G_{[n]}$ of a group $G$ are defined inductively by $G^{(0)}=G$ and $G^{(n+1)}=G^{(n)^{\prime}}$, and $G_{[0]}=G$ and $G_{[n+1]}=\left[G, G_{[n]}\right]$, for $n \geqslant 0$, respectively. We shall show that if $G$ is a $\mathrm{PD}_{3}$-group such that one of these series terminates, then the maximal quotient is strongly restricted.

Theorem 11.4 [62]. Let $P$ be a $\mathrm{PD}_{3}$-space with fundamental group $\pi$, and such that $\pi^{(n+1)}=\pi^{(n)}$ for some $n \geqslant 0$. Then we are in one of the following situations:

(1) $\pi / \pi^{(n)}$ is finite and has cohomological period dividing 4 , and $\pi$ is orientable.

(2) $\pi / \pi^{(n)}$ is isomorphic to one of $\mathbb{Z}, \mathbb{Z} \oplus(\mathbb{Z} / 2 \mathbb{Z})$ or $D_{\infty}$.

(3) $\pi / \pi^{(n)}$ is torsion-free and polycyclic, and $h\left(\pi / \pi^{(n)}\right)=3$.

In each case, $n \leqslant 3$.

Proof. Let $\kappa=\pi^{(n)}$. Then $\kappa$ is a perfect normal subgroup and $G=\pi / \kappa$ is solvable. Since $\kappa$ acts trivially on $\mathbb{Z}[G]$ and is perfect,

$$
H^{1}(\kappa ; \mathbb{Z}[G])=\operatorname{Hom}(\kappa, \mathbb{Z}[G])=0 .
$$

Hence the canonical homomorphism $H^{1}(G ; \mathbb{Z}[G]) \rightarrow H^{1}(\pi ; \mathbb{Z}[G])$ is an isomorphism.

Since $G$ is solvable it has finitely many ends. If $G$ is finite then the covering space $P_{\kappa}$ is again a $\mathrm{PD}_{3}$-complex, and so $H_{2}\left(P_{\kappa} ; \mathbb{Z}\right)=H^{1}(\kappa ; \mathbb{Z})=0$. Moreover, $P_{\kappa}$ is orientable, since $H_{1}\left(\kappa ; \mathbb{F}_{2}\right)=0$. Considering the Serre spectral sequence for the projection of $X_{\kappa}$ onto $P$ (with coefficients $\mathbb{Z}$ ), we see that $G$ acts orientably on $P_{\kappa}$ and has cohomological period dividing 4 .

Lemma 4.6 and Corollary 4.9.1 extend to show that if $G$ is infinite then it has no nontrivial finite normal subgroup other than $\mathbb{Z} / 2 \mathbb{Z}^{-}$. Hence if $G$ has two ends then it is either $\mathbb{Z}, \mathbb{Z} \oplus(\mathbb{Z} / 2 \mathbb{Z})$ or $D_{\infty}$.

If $G$ has one end then

$$
H_{2}\left(P_{\kappa} ; \mathbb{Z}\right)=H_{2}(\pi ; \mathbb{Z}[G]) \cong H^{1}(\pi ; \mathbb{Z}[G])=H^{1}(G ; \mathbb{Z}[G])=0,
$$

while $H_{3}\left(P_{\kappa} ; \mathbb{Z}\right)=0$. We may assume that $C_{*}(P ; \mathbb{Z}[\pi]) \simeq C_{*}$, where $C_{*}$ is a finite projective $\mathbb{Z}[\pi]$ chain complex of length 3 . Hence $\mathbb{Z}[G] \otimes_{\mathbb{Z}[\pi]} C_{*}$ is a finite projective resolution of the augmentation $\mathbb{Z}[G]$-module $\mathbb{Z}$. Hence $G$ is FP, and $\operatorname{cd} G \leqslant 3$. Since $H_{j}\left(P_{\kappa} ; \mathbb{Z}[G]\right)=0$ for $j>0$, we also have $H^{j}\left(P_{\kappa} ; \mathbb{Z}[G]\right)=0$ for $j>0$, by the Universal Coefficient Theorem with simple coefficients $\mathbb{Z}[G]$. Hence the Serre spectral sequence with these coefficients collapses, to give $H^{3}(G ; \mathbb{Z}[G]) \cong H^{3}(\pi ; \mathbb{Z}[G]) \cong \mathbb{Z}$. Hence $G$ is a $\mathrm{PD}_{3}$-group. Since it is solvable, it is polycyclic and of Hirsch length 3.

The final assertion holds for each possible quotient $\pi / \pi^{(n)}$.

If $G$ has two ends and $\kappa=\pi^{(n)}$ is infinite, then it is not finitely generated. For otherwise $\pi$ would have one end, and so $P$ would be aspherical. Then $\kappa$ would be a $\mathrm{PD}_{2}$-group, by the PD Extension Theorem, but no $\mathrm{PD}_{2}$-group is perfect.

Each of the possible quotients $\pi / \kappa$ is a 3-manifold group. Examples with $\kappa \neq 1$ may be constructed from such manifolds $M$ by replacing a regular neighbourhood of an essential simple closed curve by the exterior of a nontrivial knot with Alexander polynomial 1.

There is an analogous result for the lower central series.

Lemma 11.5 [140, Lemma 4]. Let $N$ be a finitely generated nilpotent group. If there is a $k \geqslant 0$ such that $H_{i}(N ; \mathbb{Z})=0$ for all $i>k$, then $N$ is torsion-free and $h(N) \leqslant k$. 
Proof. If $h(N)=0$ then $N$ is finite, and so $N=1$, since nontrivial finite groups have nonzero homology in infinitely many degrees [135]. If $h(N)>0$ then $N / N^{\prime}$ is infinite [Ro, 5.2.6], and so there is an epimorphism $f: N \rightarrow \mathbb{Z}$. Let $U_{1}=U=\operatorname{Ker}(f)$, and let $U_{k+1}=\left[N, U_{k}\right]$, for $k>0$. Then $N / U$ acts trivially on $U_{k} / U_{k+1}$, by definition. Assume that $N / U$ acts nilpotently on the homology of $U / U_{k}$. It then follows from the LHSSS for $U / U_{k+1}$ as an extension of $U / U_{k}$ by $U_{k} / U_{k+1}$ that $N / U$ acts nilpotently on the homology of $U / U_{k+1}$. Hence $N / U$ acts nilpotently on $H_{i}(U)$, for all $i \geqslant 0$.

The LHSSS for $N$ as an extension of $\mathbb{Z}$ by $U$ reduces to a long exact ("Wang") sequence

$$
H_{i}(U) \rightarrow H_{i}(U) \rightarrow H_{i}(N) \rightarrow H_{i-1}(U) \rightarrow \ldots,
$$

where $H_{i}(-)$ denotes homology with integral coefficients. Hence $H_{i}(U)=(t-1) H_{i}(U)$, for $i>k$, since $H_{i}(N)=0$ for $i>k$. But then $H_{i}(U)=(t-1)^{j} H_{i}(U)$ for all $j \geqslant 0$, and so $H_{i}(U)=0$. The lemma now follows by induction on $h(N)$.

If $N$ is torsion-free then it is a $\mathrm{PD}_{h(N)}^{+}$-group, so $H_{h(N)}(N ; \mathbb{Z}) \neq 0$, while $H_{i}(N ; \mathbb{Z})=0$ for all $i>h(N)$. Lemma [28]. Let $N$ be a finitely generated nilpotent group and $M$ a finitely generated $\mathbb{Z}[N]$-module. If $H_{0}(N ; M)=0$ then $H_{i}(N ; M)=0$ for all $i \geqslant 0$.

Let $\Gamma_{q}$ be the central extension of $\mathbb{Z}^{2}$ by $\mathbb{Z}$ with characteristic class $\pm q \in H^{2}\left(\mathbb{Z}^{2} ; \mathbb{Z}\right) \cong \mathbb{Z}$. Then $\Gamma_{q}$ has the presentation

$$
\left\langle x, y, z \mid x, y \leftrightharpoons z,[x, y]=z^{q}\right\rangle .
$$

Theorem 11.6 [140]. Let $P$ be an orientable $\mathrm{PD}_{3}$-space with fundamental group $\pi$, such that $\pi_{[n]}=$ $\pi_{[n+1]}$ for some $n$. Then we are in one of the following situations:

(1) $\pi / \pi_{[n]}$ is isomorphic to $\mathbb{Z} / m \mathbb{Z}$ or to $Q\left(2^{k}\right) \times \mathbb{Z} /(2 k+1) \mathbb{Z}$, for some $k \geqslant 3$ and $m \geqslant 1$.

(2) $\pi / \pi_{[n]} \cong \mathbb{Z}$.

(3) $\pi / \pi_{[n]}$ is isomorphic to $\mathbb{Z}^{3}$ or to $\Gamma_{q}$, for some $q \geqslant 1$.

Proof. Let $N=\pi_{[n]}$ and $G=\pi / N$. Since $G$ is nilpotent it has finitely many ends.

If $G$ is finite then it is the direct product of its Sylow $p$-subgroups. Since $G$ is the maximal nilpotent quotient of $\pi, N$ has no characteristic quotient which is a $p$-group for any $p$ dividing $|G|$. Hence $N / N^{\prime}$ is finite and $\left(|G|,\left|N / N^{\prime}\right|\right)=1$, and so the $\mathrm{PD}_{3}$-space $P_{N}$ is a $\mathbb{Z}_{(p)}$-homology 3 -sphere. It follows easily that each Sylow $p$-subgroup has cohomological period dividing 4 , and so the same is true for $G$. The finer detail in (1) then follows from inspection of the list of such groups in Chapter 5.

If $G$ has two ends then it is an extension of $\mathbb{Z}$ by a finite normal subgroup, since $D_{\infty}$ is not nilpotent. We refer to the original paper [140] for the proof that this finite subgroup is trivial, and so $G \cong \mathbb{Z}$. (We do not need this detail for the corollary below.)

If $G$ has one end then $H_{i}\left(P_{N} ; \mathbb{Z}\right)=H_{i}(P ; \mathbb{Z}[G])=0$ for $i \geqslant 2$, by Poincaré duality for $P$, while $H_{0}\left(G ; H_{1}\left(P_{N} ; \mathbb{Z}\right)\right)=N /[\pi, N]=0$. Hence $H_{i}\left(G ; H_{1}\left(P_{N} ; \mathbb{Z}\right)\right)=0$ for all $i \geqslant 0[28$, Lemma 4], and so the spectral sequence

$$
H_{p}\left(G ; H_{q}\left(P_{N} ; \mathbb{Z}\right)\right) \Rightarrow H_{p+q}(P ; \mathbb{Z})
$$

for the covering $P_{N} \rightarrow P$ collapses to give isomorphisms $H_{i}(P ; \mathbb{Z}) \cong H_{i}(G ; \mathbb{Z})$, for all $i \geqslant 0$. Since $P$ is an orientable $\mathrm{PD}_{3}$-complex, $G$ is torsion-free and $h(G)=3$, by Lemma 11.5. Hence $G$ is isomorphic to $\mathbb{Z}^{3}$ or to $\Gamma_{q}$, for some $q \geqslant 1$. 
Corollary 11.6.1. If $\beta_{1}\left(G ; \mathbb{F}_{p}\right)>1$ for an odd prime $p$ then the pro- $p$ completion of $G$ is infinite. Similarly, if $\beta_{1}\left(G ; \mathbb{F}_{2}\right)>2$ then the pro-2 completion is infinite.

There are parallel results for coefficients $\mathbb{Q}$. If the rational lower central series terminates, then $\beta=$ $\beta_{1}(G) \leqslant 3$; if, moreover, $\beta=3$ then there is a nonzero triple cup-product, while if $\beta=2$ there is a nonzero Massey product. (See $[43 ; 140]$.)

\subsection{PD $_{3}$-groups with infinite profinite completion}

In a much-cited but unpublished preprint, Mess showed that if $M$ is a closed $P^{2}$-irreducible 3-manifold and $\pi=\pi_{1}(M)$ is such that $\widehat{\pi}$ is infinite, then either $\pi$ is polycyclic, or there is a prime $p$ such that $v \beta(\pi ; p)=\infty$, and in the latter case $\pi$ has a subgroup that is a nonabelian free group [109, Proposition 3]. Here we shall give some related results on $\mathrm{PD}_{3}$-groups, from [14], as steps towards the Tits Alternative, considered in the next section. This work implies most of [109, Proposition 3] for almost coherent residually finite $\mathrm{PD}_{3}$-groups. (We discuss this briefly at the end of the chapter. The other results of [109] are similar to Corollary 11.6.1 above and Theorem 11.11 below.)

The $\operatorname{rank} \operatorname{rk}(G)$ is the minimal number of elements needed to generate the group $G$. The Prüfer rank $u(G)$ is the maximum of the ranks of all finitely generated subgroups of $G$. The sectional $p$-rank $u_{p}(G)$ is the minimal $r \in \mathbb{Z} \cup\{\infty\}$ such that the $p$-Sylow subgroups of finite quotients of $G$ all have Prüfer rank $\leqslant r$.

Lemma 11.7 [14, Lemma 2.3]. Let $G$ be a finitely generated group. If $v \beta(G ; p)$ is finite for some prime $p$ then $u_{p}(H) \leqslant v \beta(G ; p)$, for every subgroup $H$ of finite index in $G$.

Proof. Let $H<G$ have finite index in $G$ and let $f: H \rightarrow F$ be an epimorphism onto a finite group $F$. Let $S_{p}(F)$ be the $p$-Sylow subgroup of $F$, and let $d=u\left(S_{p}(F)\right)$. Then $S_{p}(F)$ maps onto $(\mathbb{Z} / p \mathbb{Z})^{d}$. Let $K=f^{-1}\left(S_{p}(F)\right)$. Then $K$ has finite index in $G$, and $K$ maps onto $(\mathbb{Z} / p \mathbb{Z})^{d}$. Hence $v \beta(G ; p) \geqslant$ $\beta_{1}\left(K ; \mathbb{F}_{p}\right) \geqslant d$. In other words, $u_{p}(H) \leqslant v \beta(G ; p)$.

If $G$ is a group and $p$ is a prime, let $X^{p}(G)=\left\langle g^{p} \mid g \in G\right\rangle$ denote the subgroup generated by all $p$-th powers in $G$. Let $G_{[0], p}=G$ and $G_{[n+1], p}=\left[G, G_{[n], p}\right] X^{p}\left(G_{[n], p}\right)$, for all $n \geqslant 0$. The $p$-lower central series $\left\{G_{[n], p}: n \geqslant 0\right\}$ is the most rapidly descending central series such that all subquotients are elementary abelian $p$-groups. If $N$ is a normal subgroup of $G$, then the LHSSS for $G$ as an extension of $G / N$ with coefficients $\mathbb{F}_{p}$ gives a 5 -term "exact sequence of low degree"

$$
H_{2}(G) \rightarrow H_{2}(G / N) \rightarrow N /[G, N] \rightarrow H_{1}(G) \rightarrow H_{1}(G / N) \rightarrow 0,
$$

where $H_{i}(-)$ now denotes homology with coefficients $\mathbb{F}_{p}$ [132].

Lemma 11.8. Let $G$ be a group and $E$ a subgroup generated by $k$ elements. Then $\beta_{1}\left(E G_{[1], p} ; \mathbb{F}_{p}\right) \geqslant$ $\operatorname{rk}\left(G_{[1], p} / G_{[2], p}\right)-\left(\begin{array}{l}k \\ 2\end{array}\right)$.

Proof. Let $F=E G_{[1], p} / G_{[1], p}$. Then $F \cong(\mathbb{Z} / p \mathbb{Z})^{r}$, for some $r \leqslant k$. Hence $\beta_{1}\left(F ; \mathbb{F}_{p}\right)=r$ and $\beta_{2}\left(F ; \mathbb{F}_{p}\right)=\left(\begin{array}{c}r+1 \\ 2\end{array}\right)$, by the Künneth Theorem. The result then follows from the exact sequence of low degree for $E G_{[1], p}$ as an extension of $F$ by $G_{[1], p}$.

Lemma 11.9. Let $G$ be an orientable $\mathrm{PD}_{3}$-group and $p$ a prime. Then $\operatorname{rk}\left(G_{[1], p} / G_{[2], p}\right) \geqslant\left(\begin{array}{c}\beta \\ 2\end{array}\right)$, where $\beta=\beta_{1}\left(G ; \mathbb{F}_{p}\right)$. If $\beta>3$ then $v \beta(G ; p)=\infty$. 
Proof. Since $G / G_{[1], p} \cong(\mathbb{Z} / p \mathbb{Z})^{\beta}$, we see that $\beta_{1}\left(G / G_{[1], p} ; \mathbb{F}_{p}\right)=\beta$ and $\beta_{2}\left(G / G_{[1], p} ; \mathbb{F}_{p}\right)=\left(\begin{array}{c}\beta+1 \\ 2\end{array}\right)$. Since $\beta_{2}\left(G ; \mathbb{F}_{p}\right)=\beta$, by Poincaré duality, it follows from the exact sequence of low degree for $G$ as an extension of $G / G_{[1], p}$ by $G_{[1], p}$ that $\operatorname{rk}\left(G_{[1], p} / G_{[2], p}\right) \geqslant\left(\begin{array}{c}\beta+1 \\ 2\end{array}\right)-\beta=\left(\begin{array}{c}\beta \\ 2\end{array}\right)$.

Clearly $G_{[1], p}^{\prime} \leqslant\left[G, G_{[1], p]}\right.$. Therefore if $\beta>3$ then

$$
\beta_{1}\left(G_{[1], p} ; \mathbb{F}_{p}\right) \geqslant \operatorname{rk}\left(G_{[1], p} / G_{[2], p}\right) \geqslant\left(\begin{array}{c}
\beta \\
2
\end{array}\right)>\beta=\beta_{1}\left(G ; \mathbb{F}_{p}\right) .
$$

Iterating this argument, we get $v \beta(G ; p)=\infty$.

Lemma 11.10 [14, Proposition 2.5]. Let $G$ be an orientable $\mathrm{PD}_{3}$-group such that $v \beta(G ; p)$ is finite for every prime $p$. Then $G$ has a subgroup $H$ of finite index such that each finite quotient of $H$ is solvable with Prüfer rank $\leqslant 4$.

Proof. The group $G$ has finite sectional 2-rank, by Lemma 11.7, and so has a finite index subgroup $H$ all of whose finite quotients are solvable [104]. On the other hand, if $S$ is a finite solvable group all of whose Sylow subgroups have Prüfer rank $\leqslant r$, then $S$ has Prüfer rank $\leqslant r+1$ [92]. The result now follows from Lemma 11.9.

Theorem 11.11 [14, Proposition 2.2]. Let $G$ be an orientable $\mathrm{PD}_{3}$-group such that $\widehat{G}$ is infinite. Then either

(1) $v \beta(G)>0$, or

(2) $v \beta(G ; p)=\infty$ for some prime $p$.

Proof. Suppose that $v \beta(G ; p)$ is finite for every prime $p$. Then $G$ has a subgroup $H$ of finite index as in Lemma 11.10. Let $K=\bigcap \mathfrak{N}$, where $\mathfrak{N}$ is the directed system of normal subgroups of finite index in $H$. Then $H / K$ is finitely generated, residually finite, and each finite quotient of $H / K$ is solvable with Prüfer rank $\leqslant 4$. Hence $H / K$ is nilpotent-by-abelian [129]. Since $H / K$ is infinite, it has a subgroup of finite index which maps onto $\mathbb{Z}$, and so $v \beta(G)>0$.

In [109, Proposition 2] it is shown that if $M$ is a closed $P^{2}$-irreducible 3-manifold, $p$ is a prime and $\pi=\pi_{1}(M)$ is such that $\widehat{\pi}_{p}$ is infinite, then either $\pi$ has an infinite solvable quotient of derived length $\leqslant 2$, in which case $v \beta(G)>0$, or $v \beta(G ; p)=\infty$. (The argument involves embedding $\widehat{\pi}_{p}$ in a $p$-adic analytic group.)

Lemma 11.12 [130, Proposition 1.1]. Let $G$ be an orientable $\mathrm{PD}_{3}$-group and $p$ a prime, and suppose that $\beta_{1}\left(G ; \mathbb{F}_{p}\right)=k+2$ for some $k \geqslant 2$. If $E$ is a subgroup of $G$ which can be generated by $k$ elements then $[G: E]=\infty$, and $E$ is contained in infinitely many distinct subgroups of finite index.

Proof. Let $\Delta<G$ be a subgroup of finite index such that $E \leqslant \Delta$ and $\beta_{1}\left(\Delta ; \mathbb{F}_{p}\right)=n \geqslant k+2$. Then $\Delta$ is an orientable $\mathrm{PD}_{3}$-group. Let $D=E \Delta_{[1], p}$. Then $D$ is a proper subgroup of $\Delta$, which has finite index and contains $E$. Moreover,

$$
\beta_{1}\left(D ; \mathbb{F}_{p}\right) \geqslant\left(\begin{array}{l}
n \\
2
\end{array}\right)-\left(\begin{array}{l}
k \\
2
\end{array}\right) \geqslant\left(\begin{array}{l}
n \\
2
\end{array}\right)-\left(\begin{array}{c}
n-2 \\
2
\end{array}\right)=2 n-3>\beta_{1}\left(\Delta ; \mathbb{F}_{p}\right),
$$

by Lemmas 11.8 and 11.9 . It follows that $E$ is contained in infinitely many such subgroups, and so $[G: E]=\infty$. 
A group is $k$-free if every subgroup generated by at most $k$ elements is a free group, and is properly locally cyclic if every finitely generated proper subgroup is cyclic.

Lemma 11.13 [14, Lemma 4.1]. Let $G$ be a $\mathrm{PD}_{3}$-group which is properly locally cyclic. Then $G$ is simple, and is generated by any two non-commuting elements.

Proof. If $N$ is a proper normal subgroup then it is abelian of rank 1 , and so $\sqrt{G} \neq 1$. Hence $G$ is either of Seifert type or is a $\mathrm{Sol}^{3}$-group, by Theorem 8.10. In each case, such groups are not properly locally cyclic. Since $G$ is simple and not cyclic, it has two non-commuting elements $x, y$, say. Since $\langle x, y\rangle \supsetneqq \mathbb{Z}$, we must have $G=\langle x, y\rangle ¥ \mathbb{Z}$, by definition of "properly locally cyclic".

Must a properly locally cyclic group be finitely presentable?

Theorem 11.14 [14, Theorem 2.1]. Let $G$ be an almost coherent $\mathrm{PD}_{3}$-group. If $\widehat{G}$ is infinite then either

(1) G contains a $\mathrm{PD}_{2}$-group, or

(2) $G$ is virtually $k$-free, for all $k \geqslant 0$.

In particular, $G$ is not virtually properly locally cyclic.

Proof. We may assume without loss of generality that $G$ is orientable. If $v \beta(G)>0$ then there is a subgroup $H<G$ of finite index which maps onto $\mathbb{Z}$. This subgroup is an HNN extension with finitely generated base $B$, by the Bieri-Strebel Theorem. Since $H$ is a $\mathrm{PD}_{3}$-group, $3=\mathrm{cd} H \leqslant \mathrm{~cd} B+1$, while since $[H: B]=\infty, \operatorname{cd} B \leqslant 2$, by the Strebel Theorem. Hence $B$ has an indecomposable factor $D$ which is finitely generated and has one end. Since $G$ is almost coherent, finitely generated subgroups are $\mathrm{FP}_{2}$, the subgroup $D$ is the ambient group of a $\mathrm{PD}_{3}$-pair of groups, and so $D$ (and hence $G$ ) has a subgroup which is a $\mathrm{PD}_{2}$-group.

Suppose that $G$ does not contain a $\mathrm{PD}_{2}$-group. Then $v \beta(G)=0$, and so there is a prime $p$ such that $v \beta(G ; p)=\infty$, by Theorem 11.11 . Let $G_{k}$ be a subgroup of finite index and such that $\beta\left(G_{k} ; \mathbb{F}_{p}\right) \geqslant k+2$, and let $H$ be a subgroup of $G_{k}$ of rank $\leqslant k$. Then $H$ has infinite index in $G_{k}$, by Lemma 11.12. Since $H$ is $\mathrm{FP}_{2}$ and $G$ does not have any subgroups which are $\mathrm{PD}_{2}$-groups, it follows from the Algebraic Core Theorem that $H$ must be free. Hence $G_{k}$ is $k$-free.

Since $G$ has normal subgroups of arbitrarily large finite index, it is not virtually properly locally cyclic, by Lemma 11.13 .

If $M$ is an irreducible orientable 3-manifold such that $\pi=\pi_{1}(M)$ has no subgroup isomorphic to $\pi_{1}\left(T_{g}\right)$ for any $g<k$, then $\pi$ is $k$-free, and finitely generated freely indecomposable subgroups of infinite index in $\pi$ have deficiency $\geqslant k$ [5]. There is an analogue of this for almost coherent $\mathrm{PD}_{3}^{+}$-groups, but we must modify the notion of deficiency.

If $G$ is an $\mathrm{FP}_{2}$ group, its augmentation ideal has presentations

$$
P \rightarrow \mathbb{Z}[G]^{r} \rightarrow I(G) \rightarrow 0
$$

where $r$ is finite and $P$ is a finitely generated projective module. Let

$$
\operatorname{def}_{\mathbb{Z}[G]}(G)=\max \left\{r-\operatorname{rank}\left(\mathbb{Z} \otimes_{\mathbb{Z}[G]} P\right)\right\},
$$

where the maximum is taken over all such presentations of $I(G)$. Then $\operatorname{def}_{\mathbb{Z}[G]}(G) \leqslant \beta_{1}(G)-\beta_{2}(G)$, while if $G$ is finitely presentable then $\operatorname{def}(G) \leqslant \operatorname{def}_{\mathbb{Z}[G]}(G)$. 
Theorem 11.15. Let $G$ be an almost coherent $\mathrm{PD}_{3}$-group, with no subgroup which is a $\mathrm{PD}_{2}^{+}$-group of genus $<k$, and let $H$ be a finitely generated freely indecomposable subgroup of $G$ such that $\mathrm{cd} H=2$. Then $\operatorname{def}_{\mathbb{Z}[H]}(H) \geqslant k$, and $G$ is $k$-free.

Proof. Since $G$ is almost coherent, $H$ is $\mathrm{FP}_{2}$. If $H$ has one end then $\mathrm{cd} H=2$, and $H$ is the ambient group of a $\mathrm{PD}_{3}$-pair of groups $(H, \mathcal{T})$, by the Algebraic Core Theorem. Since $[G: H]=\infty, \mathcal{T}$ is nonempty, and $\sum_{H \cdot \tau} \chi\left(H_{\tau}\right)=2 \chi(H)$ (where the sum is over representatives of the boundary components), by Lemma 3.1. Hence $\chi(H) \leqslant 1-k$, by the hypothesis on $G$. Since $H$ is $\mathrm{FP}_{2}$ and $c d H=2$, there is a resolution

$$
0 \rightarrow P \rightarrow \mathbb{Z}[H]^{r} \rightarrow \mathbb{Z}[H] \rightarrow \mathbb{Z} \rightarrow 0
$$

and so $\chi(H)=1-r+\operatorname{rank}\left(\mathbb{Z} \otimes_{\mathbb{Z}[G]} P\right)$. Hence

$$
\operatorname{def}_{\mathbb{Z}[H]}(H) \geqslant r-\operatorname{rank}\left(\mathbb{Z} \otimes_{\mathbb{Z}[G]} P\right)=1-\chi(H) \geqslant k
$$

If $H$ can be generated by at most $k$ elements then we may assume that $r=k$, and so $\mathbb{Z} \otimes_{\mathbb{Z}[G]} P=0$. Since cd $H=2$ it follows that $P=0$ [30]. But then cd $H=1$, contrary to assumption. It follows easily that $G$ must be $k$-free.

In the 3-manifold case we have the advantage of knowing that $K(H, 1)$ is homotopy equivalent to a finite 2-complex, by Scott's Core Theorem. Moreover, since $\pi$ is coherent the fact that $\pi$ is $k$-free is an easy consequence of [Ro, 14.1.7]. Orientability simplifies the formulation of the result, but is not otherwise necessary.

\subsection{The Tits Alternative}

A group is a Tarski monster if every proper subgroup is cyclic. Olshanskii has shown that there are finitely generated groups of cohomological dimension 2 which are Tarski monsters [O1, §§28-29], but it is not clear whether there are aspherical finite 2-complexes with such groups, nor whether these groups are finitely presentable. It is not known whether there are any $\mathrm{PD}_{3}$-groups which are Tarski monsters, or which are properly locally cyclic.

It follows from Lemma 11.13 that if a $\mathrm{PD}_{3}$-group $G$ is virtually properly locally cyclic then it does not satisfy the Tits Alternative. The main result of [14] is the following converse to this lemma, which encompasses earlier results of Parry [115] and Kochloukova and Zaleskii [89].

Theorem 11.16 [14]. Let $G$ be a $\mathrm{PD}_{3}$-group which is not virtually properly locally cyclic. If $H$ is an almost coherent restrained subgroup of $G$, then $H$ is solvable.

Proof. If $[G: H]=\infty$ then $H$ is virtually abelian, by Theorem 11.2. Thus we may assume that $[G: H]<\infty$. Then $H$ is an almost coherent $\mathrm{PD}_{3}$-group which is not virtually properly locally cyclic. If $\widehat{H}$ is finite then $H$ has a normal subgroup $K$ of maximal finite index. Since $K$ is again not virtually properly locally cyclic, it has a finitely generated proper subgroup $L$ which is not cyclic. This subgroup must have infinite index, for otherwise the intersection of its conjugates in $H$ would be a normal subgroup of index strictly greater than $[H: K]$. Hence $L \cong \mathbb{Z}^{2}$ or $L \cong \mathrm{Kb}$, by Theorem 11.2.

Thus $H$ has a subgroup $L^{+} \cong \mathbb{Z}^{2}$. By the JSJ decomposition Theorem, either $H$ is of Seifert typein which case it is virtually polycyclic, since it is restrained - or $H$ splits over a subgroup $A \cong \mathbb{Z}^{2}$. But 
it is easy to see that in the latter case $H$ must be either an extension of $D_{\infty}$ by $A$ or a semidirect product $A \rtimes \mathbb{Z}$, and so $H$ is again solvable.

If $\widehat{H}$ is infinite then the result follows from Theorem 11.11 .

Corollary 11.16.1 [14, Corollary 1.4]. If $G$ is almost coherent and either $\beta_{1}\left(G ; \mathbb{F}_{p}\right)>1$, for an odd prime $p$, or $\beta_{1}\left(G ; \mathbb{F}_{2}\right)>2$, then $G$ satisfies the Tits Alternative.

Proof. Since $\widehat{G}$ is infinite, by Corollary $11.6 .1, G$ is not virtually properly locally cyclic, by Theorem 11.11 and Lemma 11.13 .

We may use the doubling construction to settle the case for the ambient groups of $\mathrm{PD}_{3}$-pairs of groups. Corollary 11.16.2. Let $(G, \Omega)$ be a $\mathrm{PD}_{3}$-pair of groups with $\Omega$ nonempty. Assume that $G$ is almost coherent. If $H$ is a finitely generated restrained subgroup of $G$, then $H$ is virtually abelian.

Proof. We may assume that $\operatorname{cd} H=2$, for otherwise $H \cong \mathbb{Z}$ or $H=1$. Since $H$ is restrained it has one end, and since $G$ is almost coherent, $H$ is $\mathrm{FP}_{2}$. Since $H$ embeds in the $\mathrm{PD}_{3}$-group $D(G, \Omega)$ (the double of $G$ along its boundary), it is the ambient group of a $\mathrm{PD}_{3}$-pair of groups $(H, \mathcal{T})$, by the Algebraic Core Theorem. The double $D(H, \mathcal{T})$ is clearly not properly locally cyclic, since it has a finitely generated subgroup $H \nsubseteq \mathbb{Z}$. The result now follows from Theorem 11.16.

There are two further main results in [14]. (We have reformulated the statements of the next lemma and theorem.)

Lemma 11.17 [14, Lemma 4.2]. Let $G$ be a $\mathrm{PD}_{3}$-group. If $G$ is virtually properly locally cyclic then $u(G) \leqslant 3$.

Proof. Let $G_{0}$ be a properly locally cyclic subgroup of finite index in $G$. Since $G$ is simple, by Lemma 11.13 , it is normal in $G$. Let $p: G \rightarrow F=G / G_{0}$ be the quotient epimorphism. Then $F$ is a finite group with cohomological period dividing 4 . Inspection of the possibilities listed in $\S 3$ of Chapter 5 shows that $u(F) \leqslant 3$.

Let $H$ be a finitely generated subgroup of $G$. If $H \cap G_{0}$ is 1 or $\mathbb{Z}$ then $H$ is finite or has two ends. Since $G$ is torsion-free, $H$ must be cyclic, and so $\operatorname{rk}(H) \leqslant 1$. If $G_{0} \leqslant H$ then $H$ is a $\mathrm{PD}_{3}$-group. Choose elements $\left\{a_{1}, \ldots, a_{r}\right\}$ in $H$ representing a minimal generating set for $H / G_{0} \leqslant F$, and let $K=\left\langle a_{1}, \ldots, a_{r}\right\rangle$. If $K \cap G_{0}=1$ then $K=1$, so $H=G_{0}$ and $\operatorname{rk}(H)=2$, by Lemma 11.13. If $K \cap G_{0}$ is a proper subgroup of $G_{0}$, then $K \cong\langle t\rangle$ for some $t$ in $G$, and $t^{n} \in G_{0}$ for $n=\left[G: G_{0}\right]$. Choose $x \in G_{0} \backslash K$. Then $\left\langle t^{n}, x\right\rangle$ is not cyclic, and so $G_{0}=\left\langle t^{n}, x\right\rangle$, by Lemma 11.3. Hence $H=\langle t, x\rangle$ and so $\operatorname{rk}(H)=2$. Finally, if $G_{0} \leqslant K$ then $H=K$ and $\operatorname{rk}(H) \leqslant u(F)$. In all cases, $\operatorname{rk}(H) \leqslant 3$ and so $u(G) \leqslant 3$.

Theorem 11.18 [14, Theorem 1.6]. Let $G$ be a $\mathrm{PD}_{3}$-group. Then $G$ is restrained if and only if $u(G) \leqslant 3$.

Proof. If $G$ is virtually properly locally cyclic then $u(G) \leqslant 3$, by Lemma 11.17 , and $G$ is clearly restrained. Therefore we may assume that $G$ satisfies the Tits Alternative, by Theorem 11.16. Since $G$ is restrained, it must be solvable. Hence every subgroup $H$ is torsion-free polycyclic, with $h(H) \leqslant 3$. But it is easily checked that (in this case) any such subgroup can be generated by at most $h(H)$ elements. Hence $u(G) \leqslant 3$.

The final result involves other ideas, and we shall not prove it. 
Theorem [14, Theorem 1.8]. If a $\mathrm{PD}_{3}$-group $G$ has at least 3 distinct strictly irreducible PSL(2, $\left.\mathbb{C}\right)$ characters, then $\widehat{G}$ is infinite. Moreover, if $G$ is almost coherent then it has a subgroup which is a nonabelian free group.

\subsection{Largeness}

A group $G$ is $S Q$-universal if every countable group is a subgroup of a quotient of $G$, and is large if it has a subgroup of finite index which maps onto a nonabelian free group. If $G$ is SQ-universal then it has a nonabelian free subgroup, while if it is large then it is SQ-universal, $v \beta(G)=\infty$ and $v \beta(G ; p)=\infty$ for all primes $p$.

If $M$ is a compact orientable irreducible 3-manifold having at least one aspherical boundary component and $\pi=\pi_{1}(M)$ is not virtually abelian, then every finitely generated subgroup of $\pi$ is either solvable or SQ-universal; if also $\pi_{2}(M)=0$ then "SQ-universal" can be improved to "large" [17]. This strengthens the Tits conjecture for such groups. Can either statement be extended to $\mathrm{PD}_{3}$-groups or $\mathrm{PD}_{3}$-pairs of groups?

The final result of this chapter is a small step in this direction, and a partial strengthening of Theorem 11.11. It was originally proven for 3-manifolds with an embedded essential torus [105], and then extended to 3-manifolds with an embedded essential annulus [91]. Our strategy is based on the latter paper, but differs in detail.

Theorem 11.19. Let $G$ be a residually finite $\mathrm{PD}_{3}$-group with a subgroup $C \cong \mathbb{Z}^{2}$. Then $G$ is either polycyclic or large.

Proof. After passing to a subgroup of finite index in $G$, if necessary, we may assume that $G$ is orientable, and that $G$ splits over $C$ [98, Theorem C]. Since $G$ is residually finite, there is a descending sequence $\left\{\Delta_{i}\right\}_{i \geqslant 0}$ of normal subgroups of finite index in $G$ such that $\cap \Delta_{i}=1$.

Suppose first that $G$ is a free product with amalgamation $A *_{C} B$, and let $A_{i}=A /\left(A \cap \Delta_{i}\right), B_{i}=$ $B /\left(B \cap \Delta_{i}\right)$ and $C_{i}=C /\left(C \cap \Delta_{i}\right)$, for $i \geqslant 0$. These quotients are finite, and $C_{i}$ embeds in each of $A_{i}$ and $B_{i}$. The canonical epimorphisms of $A, B$ and $C$ onto these quotients induce an epimorphism from $G$ to $A_{i} *_{C_{i}} B_{i}$. This group is finite if $A_{i}$ or $B_{i}$ is equal to $C_{i}$, has two ends if $\left[A_{i}: C_{i}\right]=\left[B_{i}: C_{i}\right]=2$, and is virtually a nonabelian free group otherwise. It is easy to see that $\left[A_{i}: C_{i}\right] \leqslant\left[A_{i+1}: C_{i+1}\right]$ and $\left[B_{i}: C_{i}\right] \leqslant\left[B_{i+1}: C_{i+1}\right]$, for all $i$.

If $A_{i}=C_{i}$ for all $i$ then $A$ is residually finite abelian, and hence must be abelian. (For otherwise a nontrivial commutator would have nontrivial image in some finite quotient.) Since cd $A=2$ and $A$ is finitely generated, $A \cong \mathbb{Z}^{2}$, and so $[A: C]$ is finite. But $(A, C)$ is an orientable $\mathrm{PD}_{3}$-pair of groups, and this contradicts Lemma 8.6. (Note the remark on $w_{1}$ at the start of the proof of Lemma 8.6.) After discarding finitely many of the subgroups $\Delta_{i}$, we may assume that $\left[A_{i}: C_{i}\right]$ and $\left[B_{i}: C_{i}\right]$ are at least 2 for all $i$.

If $\left[A_{i}: C_{i}\right]=2$ for all $i$ then the intersection of all the subgroups of index 2 in $A$ is abelian, by a similar argument, and so $A$ is virtually abelian. As $A$ is finitely generated and $\operatorname{cd} A=2$, and as $(A, C)$ is a $\mathrm{PD}_{3}$-pair, we must have $A \cong \mathrm{Kb}$, and $C$ is normal in $A$. Hence if $\left[A_{i}: C_{i}\right]=\left[B_{i}: C_{i}\right]=2$ for all $i$ then $C$ is normal in $G$, which is then polycyclic.

If $\left[A_{i}: C_{i}\right]>2$ or $\left[B_{i}: C_{i}\right]>2$ for some $i$ then $G$ is large. 
If $G$ is an HNN extension $A *_{C} \varphi$ with stable letter $t$, then $t\left(C \cap \Delta_{n}\right) t^{-1}=t C t^{-1} \cap \Delta_{n}$ for all $n$, and so similar arguments show that either $C=A$, and so $G$ is polycyclic, or $G$ is large.

If $M$ is an aspherical closed 3-manifold, then $\pi=\pi_{1}(M)$ is residually finite and is polycyclic or large [AFW, Flowcharts 1 and 4]. Large groups are easily seen to have uniformly exponential growth. (These assertions include all of [108].)

An argument related to that for Theorem 11.19 shows that if a residually finite $\mathrm{PD}_{3}^{+}$-group splits over a $\mathrm{PD}_{2}$-group $C$ then either $G$ maps onto $\mathbb{Z}$ or $D_{\infty}$, or $G$ is large. Hence $v \beta(G)>0[70]$. 


\section{CHAPTER 12}

\section{Homomorphisms of nonzero degree}

Aspherical closed 3-manifolds may be partitioned into eight classes, according to the nature of the geometric pieces of the JSJ decomposition. Wang defined a directed graph $\Gamma$ whose vertices correspond to these classes and which has an edge whenever every manifold in the target class is the image of a map of nonzero degree from some manifold in the source class [153]. We shall give purely algebraic proofs of Wang's results for the cases when the atoroidal parts in a JSJ decomposition of the domain are of Seifert type, and our arguments apply to Poincaré duality groups in all dimensions. (In higher dimensions we partition $\mathrm{PD}_{n}$-groups into ten classes, in terms of properties of the JSJ decomposition [94].) In many cases we may find degree-1 homomorphisms between such groups.

We begin by showing that $\mathbb{N i l}^{3}$ - and $\widetilde{S L}$-manifolds are orientable, and automorphisms of their fundamental groups are orientation-preserving. In $§ 2$ we give Wang's partition and our extension to higher dimensions. In $\S 3$ we consider homomorphisms $\phi: G \rightarrow H$ with $G$ having a JSJ decomposition with all vertex groups of Seifert type and $H$ atoroidal, and in $\$ 4$ we assume that $G$ is of Seifert type or is solvable. In the final section we comment briefly on some related issues considered by Wang and others: cohopficity [51; 154; 155], the volume condition [157] and commensurability [111; 113]. However, our observations here are confined to $\mathrm{PD}_{n}$-groups which are either virtually polycyclic or of Seifert type.

\subsection{Automorphisms of $\mathbb{N i l}^{3}$ - and $\widetilde{S L}$-groups}

$\mathbb{N i l}^{3}$ - and $\widetilde{S L}$-manifolds are orientable, and so their groups are $\mathrm{PD}_{3}^{+}$-groups. This can be seen algebraically, as every such group has a characteristic subgroup $H$ which is a non-split central extension of a $\mathrm{PD}_{2}^{+}$-group $\beta$ by $\mathbb{Z}$. We shall show that an automorphism of such a group $H$ must be orientationpreserving. (See also [41].)

If $\phi: G \rightarrow H$ is a homomorphism of $\mathrm{PD}_{n}$-groups such that $w_{G}=w_{H} \phi$, the degree of $\phi$ is the induced homomorphism

$$
\operatorname{deg} \phi: H_{n}\left(G ; \mathbb{Z}^{w_{G}}\right) \rightarrow H_{n}\left(H ; \mathbb{Z}^{w_{H}}\right)
$$

(If $w_{G} \neq w_{H} \phi$ then we set $\operatorname{deg} \phi=0$.) The absolute value $|\operatorname{deg} \phi|$ is independent of the choice of generators for these groups.

Lemma 12.1. Let $\pi$ be a $\mathrm{PD}_{3}$-group with a central subgroup $A \cong \mathbb{Z}$ such that $\beta=\pi / A$ is a $\mathrm{PD}_{2}$-group, and let $\phi$ be an automorphism of $\pi$ such that $\phi(A)=A$. Then either $\pi$ is orientable and $\phi$ is orientationpreserving, or $\pi^{+} \cong \mathbb{Z} \times \beta^{+}$.

Proof. Let $e(\xi) \in H^{2}(\beta ; A)$ be the characteristic class of the central extension

$$
\xi: \quad 0 \rightarrow A \rightarrow \pi \rightarrow \beta \rightarrow 1
$$


If $\beta$ is nonorientable then $H^{2}(\beta ; A) \cong \mathbb{Z} / 2 \mathbb{Z}$, and so $\xi$ has image 0 in $H^{2}\left(\beta^{+} ; A\right) \cong \mathbb{Z}$. Hence the induced extension of $\beta^{+}$splits. Since $A$ is central in $\pi$ it follows that $\pi$ is nonorientable and $\pi^{+} \cong \mathbb{Z} \times \beta^{+}$.

If $\beta$ is orientable then so is $\pi$. The automorphism $\phi$ induces automorphisms $\eta$ and $\bar{\phi}$ of $A$ and $\beta$, respectively, and $\xi=\eta_{\#} \bar{\phi}^{*} \xi$. Therefore

$$
e(\xi)=(\operatorname{deg} \eta) \cdot e\left(\bar{\phi}^{*} \xi\right)=(\operatorname{deg} \eta) \cdot(\operatorname{deg} \bar{\phi}) \cdot e(\xi)=(\operatorname{deg} \phi) \cdot e(\xi),
$$

and so either $\operatorname{deg} \phi=1$ or $e(\xi)=0$.

Theorem 12.2. Let $M$ be a $\mathbb{N i l}^{3}$-manifold or an $\widetilde{S \mathbb{L}-m a n i f o l d . ~ T h e n ~} M$ is orientable, and every selfhomotopy equivalence of $M$ is orientation-preserving.

Proof. Let $G=\pi_{1}(M)$. Then $G$ is a $\mathrm{PD}_{3}$-group with a normal subgroup $\pi$ of finite index such that $\zeta \pi \cong \mathbb{Z}$ and such that $\beta=\pi / \zeta \pi$ is a $\mathrm{PD}_{2}^{+}$-group, and no such subgroup is a direct product. After replacing $\pi$ by the intersection of its images under automorphisms of $G$, if necessary, we may assume that $\pi$ is a characteristic subgroup.

Since $\pi$ is characteristic, every automorphism $\theta$ of $G$ restricts to an automorphism of $\pi$. Since $\pi$ is not virtually a product, $\left.\theta\right|_{\pi}$ is orientation-preserving, by Lemma 12.1 . Hence it acts trivially on $H^{3}(\pi ; \mathbb{Z}[\pi]) \cong H^{3}(G ; \mathbb{Z}[G])$. In particular, $G$ is orientable, and every automorphism of $G$ is orientationpreserving.

Necessary and sufficient conditions for an orientable Seifert fibred 3-manifold with infinite $\pi_{1}$ to have an orientation-reversing self-homeomorphism are given in [112].

All $\mathbb{S}^{3}$-manifolds are orientable. Lens spaces of the form $L(p, q)$ with $q^{2} \equiv-1 \bmod p$ have orientationreversing self homotopy equivalences. However, no such map also preserves the (Hopf) fibration over $S^{2}$. Self homotopy equivalences of the other $\mathbb{S}^{3}$-manifolds are orientation-preserving.

\subsection{Wang's partition}

If $G$ is any group, the subgroup $E(G)$ generated by all virtually solvable normal subgroups is elementary amenable. Hence if $G$ has finite cohomological dimension then $E(G)$ is itself virtually solvable [Hi, Theorem 1.11].

Lemma 12.3. Let $G$ be a $\mathrm{PD}_{n}$-group. If $h(E(G)) \geqslant n-1$ then $E(G)=G$.

Proof. Since $h(E(G)) \leqslant \operatorname{cd} E(G) \leqslant n$, either $\operatorname{cd} E(G)=n-1$ or $\operatorname{cd} E(G)=n$. In the first case, $\operatorname{cd} E(G)=h(E(G))$, so $E(G)$ is a duality group and has a finite $K(E(G), 1)$ complex [93]. A spectral sequence argument then shows that $G / E(G)$ has two ends. Otherwise $[G: E(G)]$ is finite, by the Strebel Theorem. In either case $G / E(G)$ is virtually solvable, and so $G=E(G)$.

If a $\mathrm{PD}_{n}$-group $G$ is virtually of Seifert type and is not virtually polycyclic, it has a normal subgroup $H$ of finite index such that $E(H)$ is virtually polycyclic and $H / E(H)$ is a $\mathrm{PD}_{2}$-group with $\chi(H / E(H))<0$. Since $E(H)$ is characteristic in $H$ it is normal in $G$, and so $G$ is itself of Seifert type. There are three $\mathrm{PD}_{4}$-groups which are virtually $\mathbb{Z}^{4}$, but are not of Seifert type [Hi, Chapter 7.7]. Thus "virtually of Seifert type" does not imply "of Seifert type" in general.

If $G$ is virtually of Seifert type and virtually polycyclic, it is of Seifert type and virtually nilpotent if $n \leqslant$ 3 , and is either virtually nilpotent or of Seifert type if $n=4$. However, if $A \in S L(2, \mathbb{Z})$ has trace $>2$ then 
the semidirect product $\mathbb{Z}^{2} \rtimes_{A} \mathbb{Z}$ is torsion-free and polycyclic of Hirsch length $\geqslant 3$, but is neither virtually nilpotent nor virtually of Seifert type. With more work it can be shown that there are polycyclic groups of Hirsch length $\geqslant 6$ which are virtually of Seifert type but are not virtually nilpotent or of Seifert type.

Define ten classes of $\mathrm{PD}_{n}$-groups as follows:

(1) $G$ is atoroidal;

(2) $G$ has a proper JSJ decomposition with at least one atoroidal vertex group;

(3) $G$ has a proper JSJ decomposition with all vertex groups of Seifert type;

(4) (a) $G$ is virtually polycyclic, but not virtually of Seifert type;

(b) $G$ is virtually a product $E \times \mathbb{Z}^{2}$, where $E$ is polycyclic but not virtually nilpotent;

(c) $G$ is virtually polycyclic and virtually of Seifert type but is neither virtually nilpotent nor of type (4b);

(5) $G$ is virtually a product $S \times \pi$ of a polycyclic group $S$ with $h(S)=n-2$ and a surface group $\pi$ with $\chi(\pi)<0$;

(6) $G$ is of Seifert type, but is neither virtually such a product nor virtually polycyclic;

(7) $G$ is virtually nilpotent, but not virtually abelian;

(8) $G$ is virtually abelian.

These classes are disjoint, and their union contains all $\mathrm{PD}_{n}$-groups $G$ such that $G$ has max-c [94]. The classification is also stable under passage to subgroups of finite index. Classes (4a), (4b), (4c), (7) and (8) consist of the virtually solvable $\mathrm{PD}_{n}$-groups, and class (4b) is empty if $n \leqslant 4$, while class (4c) is empty if $n \leqslant 3$. (It is occasionally convenient to treat $(4)=(4 a) \cup(4 b) \cup(4 c)$ as a single class. The classification could be refined further by considering higher-dimensional geometries.)

Every $\mathrm{PD}_{3}$-group has max-c, and we can be more explicit.

(1) $G$ is atoroidal;

(2) $G$ has a proper JSJ decomposition with at least one atoroidal vertex group;

(3) $\sqrt{G}=1$ and $G$ has a JSJ decomposition with all vertex groups of Seifert type;

(4) $\sqrt{G} \cong \mathbb{Z}^{2}$;

(5) $\sqrt{G} \cong \mathbb{Z}$ and $G$ is virtually a product;

(6) $\sqrt{G} \cong \mathbb{Z}$ but $G$ is not virtually a product;

(7) $G$ is virtually nilpotent, but not virtually abelian;

(8) $\sqrt{G} \cong \mathbb{Z}^{3}$ (that is, $G$ is virtually abelian).

Class (1) contains the fundamental groups of closed hyperbolic 3-manifolds and class (2) contains the groups of aspherical closed 3-manifolds with a nontrivial characteristic variety such that at least one component of the complement is hyperbolic. Class (3) consists of the groups of aspherical graph manifolds (closed 3-manifolds with a proper JSJ decomposition into Seifert fibred pieces), excepting Sol-manifolds. Classes (4), (5), (6), (7) and (8) consist of the groups of $\mathbb{S o l}^{3}-, \mathbb{R}^{2} \times \mathbb{E}^{1}-, \widetilde{\mathbb{S L}}-, \mathbb{N i l}^{3}-$ and flat 3-manifolds, respectively, by Theorem 8.10. It is not known whether classes (1) and (2) have any members which are not 3-manifold groups. These classes correspond to the eight classes of [153]. 
Let $\Gamma_{n}$ be the directed graph with vertices the set

$$
V\left(\Gamma_{n}\right)=\{1,2,3,4 \mathrm{a}, 4 \mathrm{~b}, 4 \mathrm{c}, 5,6,7,8\}
$$

and with an edge $(i, j)$ if and only if $i \neq j$ and for every group $H$ in class $(j)$ there is a $\operatorname{PD}_{n}$-group $G$ in class $(i)$ and a homomorphism of nonzero degree from $G$ to $H$. Wang showed that the corresponding graph $\Gamma$ for the fundamental groups of aspherical 3-manifolds has edges $(1, n)$ and $(2, n)$ for all $n,(3, m)$ for all $m \geqslant 3,(5,8)$ and $(6,7)$. Moreover, any homomorphism between 3-manifold groups in classes not connected by an edge in $\Gamma$ has degree 0 . (We have taken into account the Geometrization Theorem of Perelman and Thurston in stating the results of $[153 ; 154]$.)

We shall give algebraic arguments for these results, excepting the existence of edges $(1,2),(2,1)$ and $(1,3)$ and the nonexistence of an edge $(3,2)$. In verifying these assertions we may pass to subgroups of finite index whenever convenient. In particular, we may assume that all Poincaré duality groups considered are orientable, and hence that the edge groups in graph-of-groups splittings (as in cases (2) and (3)) are also orientable. We may also assume without loss of generality that the graph of groups is reduced. Then the vertex groups are nonabelian, for if $G_{v}$ is abelian then $\left\{G_{e} \mid v \in \partial e\right\}$ has two members, and the inclusions are isomorphisms. (However, $\mathrm{PD}_{3}$-groups of class (4) are virtually $\mathrm{HNN}$ extensions with base $\mathbb{Z}^{2}$.)

We shall see that when $n=4$ the only nontrivial edges emanating from any of the last five vertices of $\Gamma_{4}$ are $(5,4 c),(5,8)$ and $(6,7)$. If $n>4$ then $(4 a),(4 b),(4 c),(7)$ and (8) are terminal vertices of $\Gamma_{n}$, but there are also edges $(5,4 b),(5,8),(6,4 c)$ and $(6,7)$. (Also, many groups in class $(7)$ are degree-1 quotients of groups in class (5).)

That $\Gamma$ has edges $(1, n)$ with $n \geqslant 3$ follows, as in [153], from the fact that every closed orientable manifold has a 2-fold branched cover which is the mapping torus of a pseudo-Anasov diffeomorphism. The same argument would give an edge $(1,2)$ if all groups of class (2) were 3-manifold groups. We refer to [153] for the existence of an edge $(2,1)$ in the 3-manifold case.

\subsection{Homomorphisms with domain a graph of groups}

Let $(G, \mathcal{T})$ be a $\mathrm{PD}_{3}^{+}$-pair with $\zeta G \cong \mathbb{Z}$ and $\mathcal{T} \neq \varnothing$. Then $G \cong F(r) \rtimes \mathbb{Z}$, by Theorem 8.8 . Hence $G$ has a presentation $\left\langle x_{1}, \ldots x_{r}, t \mid t x_{i} t^{-1}=\alpha\left(x_{i}\right)\right\rangle$, for some $\alpha \in \operatorname{Aut}(F(r))$. Let $\widetilde{G}=\left\langle x_{1}, \ldots x_{r}, y, t\right| t x_{i} t^{-1}=$ $\left.\alpha\left(x_{i}\right), t y=y t\right\rangle$ and $\widehat{G}=\widetilde{G} *_{\mathbb{Z}^{2}} \pi 4_{1}$, where $t$ and $y \in \widetilde{G}$ are identified with a meridian and longitude in the figure-eight knot group $\pi 4_{1}$, respectively. Then $(\widehat{G}, \mathcal{T})$ is a $\mathrm{PD}_{3}$-pair, and the natural epimorphism from $\hat{G}$ to $G \cong \hat{G} /\left\langle\left(\pi 4_{1}\right)^{\prime}\right\rangle$ induces a degree-1 map of pairs. It follows easily that $\Gamma$ has an edge $(2,3)$.

If $G$ is a $\mathrm{PD}_{3}$-group in class (4) we may assume that $\widetilde{G} \cong \mathbb{Z}^{2} \times_{\theta} \mathbb{Z}$, where $\theta \in \operatorname{SL}(2, \mathbb{Z})$ has infinite order. The automorphism $\theta$ lifts to an automorphism $\Theta$ of the free group $F(x, y)$, such that $\Theta([x, y])=[x, y]$, by a theorem of Nielsen [MKS, Section 3.5]. Let

$$
\left(G_{1}, \mathbb{Z}^{2}\right)=(F(a, b) \times \mathbb{Z},\langle[a, b]\rangle \times \mathbb{Z})
$$

and

$$
\left(G_{2}, \mathbb{Z}^{2}\right)=\left(F(x, y) \times_{\Theta} \mathbb{Z},\langle[x, y]\rangle \times \mathbb{Z}\right) .
$$

Then $G_{1} *_{\mathbb{Z}^{2}} G_{2}$ is a group in class (2) which maps onto $\widetilde{G}$ via a degree-1 homomorphism. Hence $\Gamma$ has 
an edge $(2,4)$. (We shall see below that $\Gamma$ has an edge $(3,4)$, so composition gives an edge $(2,4)$, but this construction is simpler).

The argument excluding $(3,1)$ as an edge applies in all dimensions.

Theorem 12.4. Let $G$ and $H$ be $\mathrm{PD}_{n}$-groups such that $G$ has a proper JSJ decomposition with all vertex groups of Seifert type and such that $H$ is atoroidal. If $\phi: G \rightarrow H$ is a homomorphism, then $\operatorname{deg} \phi=0$.

Proof. We may assume that $G$ is orientable. Let $(\mathcal{G}, \Gamma)$ be the graph of groups corresponding to the JSJ decomposition of $G$. Let $s_{e}: G_{e} \rightarrow G_{o(e)}$ and $t_{e}: G_{e} \rightarrow G_{t(e)}$ be the canonical inclusions of the edge group $G_{e}$ into the adjacent vertex groups. Let $\overline{\mathcal{G}}$ be the graph of groups with underlying graph $\Gamma$ and with $\bar{G}_{v}=\phi\left(G_{v}\right)$ and $\bar{G}_{e}=\phi\left(G_{e}\right)$, for all $v \in V(\Gamma)$ and $e \in E(\Gamma)$. Then $\phi$ factors as $\phi=\bar{\phi} \rho$, where $\rho: G \rightarrow \bar{G}=\pi(\overline{\mathcal{G}})$ and $\bar{\phi}: \bar{G} \rightarrow H$.

Let $E_{v}=E\left(G_{v}\right)$ and $K_{v}=\operatorname{Ker}\left(\left.\phi\right|_{E_{v}}\right)$, for all $v \in V(\Gamma)$. Then $\operatorname{cd} E_{v}=\operatorname{cd} G_{v}-1$, and so $G_{v} / E_{v}$ is virtually free [Bi, Theorem 8.4]. Suppose that $K_{v}=1$. Then $\operatorname{cd} \phi\left(G_{v}\right) \geqslant n-2$. If $\operatorname{cd} \phi\left(G_{v}\right)=n$ then $\phi\left(G_{v}\right)$ is a $\mathrm{PD}_{n}$-group, and so $H^{2}\left(\phi\left(G_{v}\right) / \phi\left(E_{v}\right) ; \mathbb{Z}\right) \cong \mathbb{Z}$, by an LHSSS corner argument. Hence $\phi\left(G_{v}\right) / \phi\left(E_{v}\right)$ is virtually a $\mathrm{PD}_{2}$-group, by the Bowditch Theorem. Similarly, if $\operatorname{cd} \phi\left(G_{v}\right)=n-1$ then $\phi\left(G_{v}\right) / \phi\left(E_{v}\right)$ is virtually free. In either case, $\phi\left(G_{v}\right) / \phi\left(E_{v}\right)$ would contain an element of infinite order. This would contradict $H$ being atoroidal. Hence $\operatorname{cd} \phi\left(G_{v}\right)=n-2$. Therefore, if $K_{v}=1$ for all $v \in V(\Gamma)$ then $\operatorname{cd} \bar{G} \leqslant n-1$, and so $\operatorname{deg} \phi=0$.

Suppose that $K_{v} \neq 1$. Let $G_{v}^{*}$ be a normal subgroup of finite index in $G_{v}$ which contains $E_{v}$ and is such that $G_{v}^{*} / E_{v}$ is a free group. Conjugation by coset representatives for $G_{v} / G_{v}^{*}$ determines lifts of the embeddings $s_{e}$ and $t_{e}$ to embeddings of $s_{e}\left(G_{e}\right) \cap G_{v}^{*}$ and $t_{e}\left(G_{e}\right) \cap G_{v}^{*}$ in $G_{v}^{*}$. Let $\mathcal{T}_{v}^{*}$ be the set of $G_{v}^{*}$-conjugacy classes of such lifts. Then $\left(G_{v}^{*}, \mathcal{T}_{v}^{*}\right)$ is a $\mathrm{PD}_{n}^{+}$-pair, and the inclusion of $G_{v}^{*}$ into $G_{v}$ has degree $\left[G_{v}: G_{v}^{*}\right] \neq 0$ (by the relative version of Lemma 8.5).

Let $\hat{G}_{v}=G_{v}^{*} / K_{v}$ and let $\widehat{\mathcal{T}}_{v}$ be the corresponding set of embeddings of quotients of members of $\mathcal{T}_{v}^{*}$. Since $E_{v} / K_{v}$ is isomorphic to $\phi\left(E_{v}\right)$, it is torsion-free, and so is a $\mathrm{PD}_{m}$-group for some $m<h\left(E_{v}\right)=n-2$. Then $\left(\hat{G}_{v}, \widehat{\mathcal{T}}_{v}\right)$ is a $\mathrm{PD}_{m+2}$-pair. Let $\overline{\mathcal{T}}_{v}=\left\{\bar{G}_{e} \mid s(e)=v\right\} \coprod\left\{\bar{G}_{e} \mid t(e)=v\right\}$, for each $v \in V(\Gamma)$.

Now $\left.\rho\right|_{G_{v}^{*}}$ factors through $\left(\hat{G}_{v}, \widehat{\mathcal{T}}_{v}\right)$, and so the homomorphism from $H_{n}\left(G_{v}^{*}, \mathcal{T}_{v}^{*} ; \mathbb{Z}\right)$ to $H_{n}\left(\bar{G}_{v}, \overline{\mathcal{T}}_{v} ; \mathbb{Z}\right)$ induced by $\left.\rho\right|_{G_{v}^{*}}$ is 0 . This homomorphism is the top row of a commuting square whose bottom row is $H_{n}(\rho ; \mathbb{Z})$. Since the inclusion of $G_{v}^{*}$ into $G$ induces an isomorphism $H_{n}\left(G_{v}^{*}, \mathcal{T}_{v}^{*} ; \mathbb{Z}\right) \rightarrow H_{n}(G ; \mathbb{Z})[8]$, it follows that $H_{n}(\rho ; \mathbb{Z})=0$. Hence we again have $\operatorname{deg} \phi=0$.

Corollary 12.4.1. There is no edge $(3,1)$ in $\Gamma_{n}$.

In the 3-manifold case it follows that there is no edge $(3,2)$. Can the above argument be adapted to show this is true in general?

Let $G$ be a torsion-free virtually polycyclic group of Hirsch length $n \geqslant 3$. Then $G$ has a subgroup $\widetilde{G}$ of finite index which is an extension of a free abelian group $\mathbb{Z}^{r}$ by a nilpotent normal subgroup $N$ [Ro, 15.1.6]. If $r=1$ then $\widetilde{G} \cong N \rtimes_{\alpha} \mathbb{Z}$, for some $\alpha \in \operatorname{Aut}(N)$. Since $N$ is nilpotent and $h(N) \geqslant 2$, there is a subgroup $P<N$ containing $N^{\prime}$ and such that $N / P \cong \mathbb{Z}^{2}$. Let $F=\langle w, x, y, z \mid w=x y z\rangle$ and $\hat{F}=\left\langle F, t \mid t w t^{-1}=z\right\rangle$ be the fundamental groups of the quadruply punctured sphere and the twice punctured torus. Let $\theta$ be the automorphism of $\hat{F}$ defined by $\theta(f)=f$ for all $f \in F$ and $\theta(t)=z t$, and let $H=\hat{F} \rtimes_{\theta} \mathbb{Z}$. (Thus $H$ is the fundamental group of the mapping torus of the Dehn twist corresponding to $\theta$.) Let $J \cong F \times \mathbb{Z}$ be the subgroup of $H$ generated by $F$ and $s$, and let $J_{f} \cong \mathbb{Z}^{2}$ be the subgroup of $J$ generated by $\{f, s\}$, for all $f \in F$. Then $H$ is also the HNN extension with base $H$, associated subgroups 
$J_{w}$ and $J_{z}$, and stable letter $t$, since $t s t^{-1}=z^{-1} s$ and $t w t^{-1}=z$. Moreover, $\left(J,\left\{J_{w}, J_{x}, J_{y}, J_{z}\right\}\right)$ is a $\operatorname{PD}_{3^{-}}$ pair of Seifert type, while $\left(H,\left\{J_{x}, J_{y}\right\}\right)$ is a $\mathrm{PD}_{3}$-pair with $\sqrt{H}=1$. Let $\gamma: H \rightarrow H /\langle t, z\rangle \cong \mathbb{Z}^{2}$ be the canonical epimorphism. Then $\gamma$ induces a degree-1 homomorphism from $\left(H,\left\{J_{x}, J_{y}\right\}\right)$ to $\left(\mathbb{Z}^{2},\left\{\mathbb{Z}^{2}, \mathbb{Z}^{2}\right\}\right)$. In particular, $\gamma$ induces isomorphisms $J_{x} \cong N$ and $J_{y} \cong N$. Let $M$ be the extension of $H$ by $P$ obtained by pullback over $\gamma$, and let $M_{x}$ and $M_{y}$ be the preimages in $M$ of $J_{x}$ and $J_{y}$, respectively. Let $\mu_{x}: M_{x} \cong N$ and $\mu_{y}: M_{y} \cong N$ be the isomorphisms determined by $\theta$. The HNN extension $G^{*}$ with base $M$, associated subgroups $M_{x}$ and $M_{y}$ and stable letter $u$ acting via $u m u^{-1}=\mu_{y}^{-1} \alpha \mu_{x}(m)$ for all $m \in M_{x}$ is a $\mathrm{PD}_{n}$-group in class (3), and the projection onto $G^{*} /\langle x, y\rangle \cong \widetilde{G}$ has degree 1 . Thus $\Gamma_{n}$ has an edge $(3,4 \mathrm{a})$. If $r \geqslant 2$ then $\widetilde{G}$ is of Seifert type, and we shall treat this case in the next paragraph.

Let $H$ be an extension of a $\mathrm{PD}_{2}^{+}$-group $\pi$ of genus $g$ by a torsion-free virtually polycyclic group $E$ of Hirsch length $n-2$. Let $F$ be the free group with basis $\left\{a_{i}, b_{i} \mid 1 \leqslant i \leqslant g\right\}$. Then $\pi \cong F /\left\langle\prod\left[a_{i}, b_{i}\right]\right\rangle$ and $H \cong E \rtimes_{\alpha} F /\left\langle\prod w^{-1} \prod\left[a_{i}, b_{i}\right]\right\rangle$, for some homomorphism $\alpha: F \rightarrow \operatorname{Aut}(E)$ and element $w \in E$ such that $\alpha\left(\prod\left[a_{i}, b_{i}\right]\right)(e)=w e w^{-1}$. Let $H_{1}=E \rtimes_{\alpha} F$ and let $\partial H_{1}$ be the subgroup generated by $E$ and $\prod\left[a_{i}, b_{i}\right]$. Then $\partial H_{1} \cong E \times \mathbb{Z}$, where the second factor is generated by $w^{-1} \prod\left[a_{i}, b_{i}\right]$. Let $H_{2}=E \times F(r, s)$ and let $\partial H_{2}=E \times \mathbb{Z}$, where the infinite cyclic factor is generated by $[r, s]$. Then $\left(H_{1}, \partial H_{1}\right)$ and $\left(H_{2}, \partial H_{2}\right)$ are $\mathrm{PD}_{n}$-pairs, with isomorphic boundary terms. Define an isomorphism $\psi: \partial H_{1} \rightarrow \partial H_{2}$ by $\psi(e)=e[r, s]$ for $e \in E$ and $\psi\left(\prod\left[a_{i}, b_{i}\right]\right)=[r, s] w$. If we identify the boundaries via $e \mapsto e$ for all $e \in E$ and $w^{-1} \prod\left[a_{i}, b_{i}\right]$ maps to $[r, s]$, we obtain a $\mathrm{PD}_{n}$-group $G$ with a nontrivial JSJ decomposition of Seifert type, but which is not itself of Seifert type. The canonical epimorphism from $G$ to $G /\langle r, s\rangle \cong H$ has degree 1 . Since every $\mathrm{PD}_{n}$-group of Seifert type has such a subgroup $H$ of finite index, it follows that $\Gamma_{n}$ has edges $(3,4 \mathrm{~b}),(3,4 \mathrm{c}),(3,5)$ and $(3,6)$. When $n=3$ this construction can be adapted to show that every group in class (5) or (6) is the degree-1 quotient of a group in class (3).

\subsection{Homomorphisms with solvable or Seifert domain}

If $E(G) \neq G$ then $h(E(G)) \leqslant n-2$, by Lemma 12.3. If $n=3$ and $E(G) \neq G$ then $E(G)=\sqrt{G}$, so that $E(G) \cong \mathbb{Z}$ or $E(G) \cong 1$, by Theorem 8.10. If $n=4$ and $h(E(G))=2$ then $E(G) \cong \mathbb{Z}^{2}$ or $E(G) \cong \mathrm{Kb}$, and $G / E(G)$ is virtually a $\mathrm{PD}_{2}$-group [Hi, Theorems 9.1 and 9.2]. To what extent can this be generalized? If $E(G)$ is of type FP and $h(E(G))=n-2$ then the LHS spectral sequence collapses to give $H^{n-2}(E(G) ; \mathbb{Z}[E(G)]) \cong H^{2}(G / E(G) ; \mathbb{Z}[G / E(G)]) \cong \mathbb{Z}$, so $E(G)$ is virtually polycyclic and $G / E(G)$ is virtually a $\mathrm{PD}_{2}$-group, by the Bowditch Theorem. If $G / E(G)$ is virtually a $\mathrm{PD}_{2}$-group then $h(E(G))=$ hd $E(G)=n-2$; must $E(G)$ be virtually polycyclic?

Lemma 12.5. Let $G$ and $H$ be $\mathrm{PD}_{n}$-groups and $\phi: G \rightarrow H$ a homomorphism such that $\operatorname{deg} \phi \neq 0$. If $K$ is a virtually polycyclic normal subgroup of $G$ and $\operatorname{ved}(G / K)$ is finite, then $\left.\phi\right|_{K}$ is a monomorphism and $\phi(K) \leqslant E(H)$. Hence if $G$ is virtually polycyclic then so is $H$, and $G$ is virtually abelian (respectively, nilpotent) if and only if $H$ is.

Proof. The image $\phi(K)$ is torsion-free and virtually polycyclic, since $H$ is torsion-free and $K$ is virtually polycyclic. If $\operatorname{Ker}\left(\left.\phi\right|_{K}\right) \neq 1$ then $\phi$ factors through $\bar{G}=G / \operatorname{Ker}\left(\left.\phi\right|_{K}\right)$, and

$$
\operatorname{cd} \bar{G}=h(\phi(K))+\operatorname{vcd}(G / K)<n=\operatorname{cd} G=h(K)+\operatorname{vcd}(G / K),
$$

since $K$ and $\phi(K)$ are $\mathrm{FP}$ and $\operatorname{vcd}(G / K)$ is finite [Bi, Theorem 5.6]. Thus $\phi$ is a monomorphism if $\operatorname{deg} \phi \neq 0$. Since $\phi(E(G))$ is a characteristic subgroup of $G$ and $\phi(G)$ has finite index in $H$, it follows 
easily that $\phi(E(G)) \leqslant E(H)$. In particular, $\phi(K) \leqslant E(H)$. The final assertion is clear.

Corollary 12.5.1. There are no edges emanating from the vertices (4a), (4b), (4c), (7) or (8) in $\Gamma_{n}$.

Lemma 12.6. Let $G$ and $H$ be $\mathrm{PD}_{n}$-groups and $\phi: G \rightarrow H$ a homomorphism such that $\operatorname{deg} \phi \neq 0$, and suppose that $G / E(G)$ is virtually a $\mathrm{PD}_{2}$-group.

(1) If $G$ is virtually a product of a solvable group with a $\mathrm{PD}_{2}$-group, then so is $H$.

(2) $G$ is virtually such a product if $\left.\phi\right|_{E(G)}$ is a monomorphism and $H$ is either virtually abelian or virtually a product of a solvable group with a nonsolvable $\mathrm{PD}_{2}$-group.

Proof. Let $\widetilde{G} \leqslant G$ be a subgroup of finite index in $G$ such that $\phi(\widetilde{G})$ is normal in $H$ and $\pi=\widetilde{G} / E(\widetilde{G})$ is a $\mathrm{PD}_{2}$-group. Note that $E(G / E(G))=1$, so $\pi, \widetilde{G}$ and $G$ are not solvable.

If $\widetilde{G} \cong E(\widetilde{G}) \times \pi$, where $\pi$ is a $\mathrm{PD}_{2}$-group, then $E(G)$ is a $\mathrm{PD}_{n-2}$-group, and hence is virtually polycyclic [Bi, Theorems 9.11 and 9.23]. Hence $\left.\phi\right|_{E(G)}$ is a monomorphism, by Lemma 12.5. As $\phi(\widetilde{G}) \cong E(G) \times \phi(\pi)$ has finite index in $H$, the latter group is also virtually a product, and $\phi(\pi)$ is a $\mathrm{PD}_{2}$-group.

If $H$ is virtually abelian, then on passing to subgroups of finite index we may assume that $\widetilde{H}$ is abelian, hence free of finite rank, and that $\phi(E(\widetilde{G}))$ is a direct factor of $\widetilde{H}$. If $H$ has a subgroup $\widetilde{H} \cong S \times \sigma$ of finite index, with $S$ solvable and $\sigma$ a nonsolvable $\operatorname{PD}_{2}$-group, then $S=E(\widetilde{H}), S$ is virtually polycyclic and $h(S)=n-2$. Moreover $\phi(E(\widetilde{G})) \leqslant S$. In either case, composition of $\phi$ with projection onto a factor splits the inclusion of $E(\widetilde{G}) \cap \phi^{-1}(\widetilde{H})$ into $\phi^{-1}(\widetilde{H})$, and so $G$ is virtually such a product.

If a $\mathrm{PD}_{n}$-group is a nontrivial direct product, its factors are $\mathrm{PD}_{m}$-groups for suitable $m<n$.

Corollary 12.6.1. If $n=3$ or 4 then $(5,4 \mathrm{c}),(5,8)$ and $(6,7)$ are the only edges emanating from the vertices (5) and (6) in $\Gamma_{n}$.

Proof. Let $G$ and $H$ be $\mathrm{PD}_{n}$-groups such that $G / E(G)$ is virtually a $\mathrm{PD}_{2}$-group, and let $\phi: G \rightarrow H$ be homomorphism such that $\operatorname{deg} \phi \neq 0$. Then $E(H) \neq 1$, by Lemma 12.5, so $H$ is not in classes (1)-(3).

If $n=3$ then $E(G) \cong \mathbb{Z}$ and so $\phi(G)$ is virtually nilpotent. Hence $H$ is virtually nilpotent. If $n=4$ then $|h(E(G))|=2$, so $h(E(G)) \cong \mathbb{Z}^{2}$ or $\mathrm{Kb}$. Therefore, if $H$ is not virtually nilpotent, it is of Seifert type and not virtually a product with $\mathbb{Z}^{2}$. The other exclusions follow easily from Lemma 12.6.

Groups in classes (7) and (8) are extensions of flat 2-orbifold groups by an infinite cyclic normal subgroup. Given such a group $G$ we may construct a group of type (6) or (5), respectively, and a degree1 homomorphism to $G$ by pulling back the extension over an epimorphism corresponding to a degree-1 map from a hyperbolic 2-orbifold. (This construction could be paraphrased in purely algebraic terms, but at somewhat greater length.)

If $n>4$ then (4a), (4b), (4c), (7) and (8) are terminal vertices of $\Gamma_{n}$, but there are also edges $(5,4 b)$, $(5,8),(6,4 c)$ and $(6,7)$, and many groups in class $(7)$ are degree-1 quotients of groups in class (5).

\subsection{Endomorphisms and subgroups of finite index}

A group $G$ is hopfian if surjective endomorphisms of $G$ are automorphisms, and is cohopfian if injective endomorphisms are automorphisms. The volume condition holds for $G$ if whenever $H_{1}$ and $H_{2}$ are isomorphic subgroups of finite index, then $\left[G: H_{1}\right]=\left[G: H_{2}\right]$. If $G$ is a $\mathrm{PD}_{n}$-group and satisfies the volume condition, then $G$ is cohopfian, since injective endomorphisms must have image of finite index, 
by the Strebel Theorem. On the other hand, finitely generated nonabelian free groups satisfy the volume condition but are not cohopfian.

If $\pi=\mathbb{Z}^{2}$ or $\pi=\mathrm{Kb}$ then $\pi$ is hopfian, by Lemma 12.5. The hopficity of the other $\mathrm{PD}_{2}$-groups follows from the next lemma, which is based on a variation of the argument given for [29, Theorem A].

Lemma 12.7. Let $\pi, \sigma$ be $\mathrm{PD}_{2}$-groups with $\chi(\sigma) \leqslant \chi(\pi)<0$, and let $\theta: \pi \rightarrow \sigma$ be a homomorphism. The following conditions are equivalent:

(1) $H_{1}\left(\theta ; \mathbb{F}_{2}\right)$ is an epimorphism.

(2) $\theta^{*} w_{\sigma}=w_{\pi}$ and $\operatorname{deg} \theta \neq 0$.

(3) $\theta$ is an isomorphism.

Proof. If $H_{1}\left(\theta ; \mathbb{F}_{2}\right)$ is an epimorphism it is an isomorphism, since $\chi(\pi)=\chi(\sigma)$, and so $\theta^{*} w_{\sigma}=w_{\pi}$, by the nondegeneracy of Poincaré duality and the Wu relation $x^{2}=x \cup w_{G}$, for $x \in H^{1}\left(G ; \mathbb{F}_{2}\right)$ and $G=\pi$ or $G=\sigma$. In particular, $\sigma \cong \pi$. Hence $\theta(\pi)$ is not a free group, so $[\sigma: \theta(\pi)]<\infty$ and $\theta(\pi)$ is a $\mathrm{PD}_{2}$-group. Since $\beta_{1}\left(\theta(\pi) ; \mathbb{F}_{2}\right) \leqslant \beta_{1}\left(\pi ; \mathbb{F}_{2}\right)=\beta_{1}\left(\sigma ; \mathbb{F}_{2}\right)$ and $\chi(\theta(\pi))=[\sigma: \theta(\pi)] \chi(\sigma)<0$, it follows that $[\sigma: \theta(\pi)]=1$, so $\theta$ is onto.

If $\theta^{*} w_{\sigma}=w_{\pi}$ and $\operatorname{deg} \theta \neq 0$, then $\sigma \cong \pi,[\sigma: \theta(\pi)]<\infty$ and $\theta$ is onto, as before.

We shall henceforth fix an isomorphism $\sigma \cong \pi$ and view $\theta$ as an endomorphism of $\pi$. If $\theta$ is onto then $H_{1}(\theta ; \mathbb{Z})$ is an isomorphism, since $H_{1}(\pi ; \mathbb{Z})=\pi / \pi^{\prime}$ is finitely generated. The induced homomorphism $\theta^{\prime}: \pi^{\prime} \rightarrow \pi^{\prime}$ is also onto, and induces an onto endomorphism of $\pi^{\prime} / \pi^{\prime \prime}$. The latter group is finitely generated as a module over the noetherian ring $\mathbb{Z}\left[\pi / \pi^{\prime}\right]$. Since the kernel $K_{n}$ of the endomorphism induced by $\theta^{n}$ is a normal subgroup of $\pi / \pi^{\prime \prime}$, it is a $\mathbb{Z}\left[\pi / \pi^{\prime}\right]$-submodule. The increasing sequence of submodules $K_{n}$ must stabilize, since $\pi^{\prime} / \pi^{\prime \prime}$ is noetherian. Hence $K_{n}=0$ for all $n$ and so $H_{1}\left(\theta^{\prime} ; \mathbb{Z}\right)$ is also an isomorphism. Now cd $\pi^{\prime} \leqslant 1$ and so $\pi^{\prime}$ is free. Hence the endomorphisms induced on the nilpotent quotients $\pi^{\prime} / \pi_{[n]}^{\prime}$ by $\theta$ are isomorphisms for all $n \geqslant 1[132]$. Hence $\operatorname{Ker}(\theta) \leqslant \bigcap_{n \geqslant 1} \pi_{[n]}^{\prime}$. Since $\pi^{\prime}$ is free it is residually a finite $p$-group [Ro, 6.1.9]. Therefore $\operatorname{Ker}(\theta)=\operatorname{Ker}\left(\theta^{\prime}\right)=1$, so $\theta$ is an automorphism. Thus (1) and (2) each imply (3); the converse is clear.

The fundamental groups of 3-manifolds are residually finite, and thus hopfian [AFW]. Hence degree-1 self maps of such groups are automorphisms.

The cohopficity of surface groups other than $\mathbb{Z}^{2}$ or $\mathrm{Kb}$ is an easy consequence of the multiplicativity of the Euler characteristic in finite extensions. This extends to all hyperbolic 2-orbifold groups.

Lemma 12.8. Let $B, C$ be closed 2-orbifolds, with orbifold fundamental groups $\rho=\pi^{\text {orb }}(B)$ and $\sigma=$ $\pi^{\mathrm{orb}}(C)$. If $\chi(\sigma) \leqslant \chi(\rho)<0$ and $\theta: \rho \rightarrow \sigma$ is a homomorphism such that $[\sigma: \theta(\rho)]<\infty$, then $\theta$ is an isomorphism. In particular, $\rho$ is both cohopfian and hopfian.

Proof. We may assume that $\sigma$ is a $\mathrm{PD}_{2}$-group, after passing to finite covers, if necessary. Let $\kappa$ be the normal subgroup of $\rho$ generated by its elements of finite order. The group $\bar{\rho}=\rho / \kappa$ is the fundamental group of the surface obtained by replacing neighbourhoods of the singular points with discs. (We do this also for annular regions bounded by reflector curves.) Hence $\chi(\rho) \leqslant \chi(\bar{\rho})$, with equality only if $\kappa=1$. Since $\sigma$ is torsion-free, $\theta$ factors through $\bar{\rho}$, and $\chi(\bar{\rho})<0$ since $\chi(\sigma)<0$ and $[\sigma: \theta(\rho)]<\infty$. Clearly $\chi(\theta(\rho)) \leqslant \chi(\sigma) \leqslant \chi(\rho) \leqslant \chi(\bar{\rho})$, and so $\bar{\rho} \cong \theta(\rho)$, by Lemma 12.7. Hence $\chi(\theta(\rho))=\chi(\sigma)=\chi(\rho)=$ $\chi(\bar{\rho})$, so $\rho$ is a $\mathrm{PD}_{2}$-group and $\theta$ is an isomorphism. The final assertion is clear. 
Lemmas 12.5 and 12.7 together imply that virtually polycyclic groups and $\mathrm{PD}_{n}$-groups of Seifert type are hopfian. On the other hand, it is easy to see that many groups in classes (4), (5), (7) and (8) are not cohopfian.

Theorem 12.9. Let $G$ be a $\mathrm{PD}_{n}$-group which is either of Seifert type, with hyperbolic base 2-orbifold, or is virtually polycyclic. Then an endomorphism $\theta: G \rightarrow G$ such that $\operatorname{deg} \theta \neq 0$ is a monomorphism.

Proof. We may assume that $G$ is of Seifert type with hyperbolic base 2-orbifold and that $\left.\theta\right|_{E(G)}$ is a monomorphism, by Lemma 12.5 . The quotient $\bar{G}=G / E(G)$ is virtually a $\mathrm{PD}_{2}$-group and has no nontrivial finite normal subgroup, and the induced homomorphism $\bar{\theta}: \bar{G} \rightarrow \bar{G}$ has image of finite index. Therefore $\bar{\theta}$ is an automorphism, by Lemma 12.8 , and so $\theta$ is a monomorphism.

Corollary 12.9.1. An endomorphism $\theta$ is an automorphism if and only if $|\operatorname{deg} \theta|=1$.

Proof. If $|\operatorname{deg} \theta|=1$ then $\theta$ is onto, by Lemma 8.5, and so is an automorphism, by the theorem. The other implication is clear.

Every sequence of degree-1 maps between geometric 3-manifolds eventually becomes a sequence of homotopy equivalences [120]. This remains true for sequences of homomorphisms between $\mathrm{PD}_{n}$-groups which contain a term from one of the classes (4)-(8), by Lemmas 12.5 and 12.6 and Corollary 12.9.1, and the fact that Euler characteristics of $\mathbb{H}^{2}$-orbifolds are bounded above (by $-\frac{1}{42}$ ). Rong handles the other cases in dimension 3 using a measure of complexity based on the Gromov norm and the number of Seifert parts.

Wang showed that every endomorphism of nonzero degree of the group of an aspherical 3-manifold is a monomorphism, and established the cohopficity of such groups in classes (1), (2), (3) and (6). He uses the Gromov norm to handle classes (1) and (2); class (3) is the most demanding [154]. We shall verify only that $\mathrm{PD}_{3}$-groups in class (6) are cohopfian.

Theorem 12.10. Let $G$ be a $\mathrm{PD}_{3}$-group such that $\sqrt{G} \cong \mathbb{Z}$ and which is not virtually a product. Then $G$ is cohopfian.

Proof. Let $\phi: G \rightarrow G$ be a monomorphism and let $\bar{G}=G / \sqrt{G}$. Then the induced endomorphism $\bar{\phi}: \bar{G} \rightarrow \bar{G}$ is an automorphism, by Lemma 12.8 . The quotient $\bar{G}$ has a normal subgroup $\bar{H}$ of finite index which is a $\mathrm{PD}_{2}^{+}$-group such that $\chi(\bar{H})<0$, and such that $\sqrt{G}$ is a central subgroup of the preimage $H \leqslant G$. Since the automorphism $\bar{\phi}$ permutes the finitely many (torsion-free) subgroups of $\bar{G}$ of index $[\bar{G}: \bar{H}]$, there is an $n \geqslant 1$ such that $\bar{\phi}^{n}(\bar{H})=\bar{H}$. Hence $\phi^{n}(H) \leqslant H$. Such extensions are classified by elements $e \in H^{2}(\bar{H} ; \mathbb{Z}) \cong \mathbb{Z}$, and it is not hard to see that $e=\left[\sqrt{G}: \phi^{n}(\sqrt{G})\right] e$. Hence either $\sqrt{G}=\phi(\sqrt{G})$, so $\phi$ is an automorphism, or $e=0$, in which case $H$ is a product and $G$ is in class (5).

The Euler class $e$ is used in a similar way in $[51 ; 154 ; 155]$. It is easy to see that no $\mathrm{PD}_{3}$-group in class (4) is cohopfian, and a similar result is proven in [51] for classes (5), (7) and (8). (See also [GW] and [50] for cohopficity of groups of 3-manifolds, with and without boundary). In higher dimensions, it is not clear whether there are any cohopfian $\mathrm{PD}_{n}$ groups in classes (4)-(8). (Central extensions of $\mathrm{PD}_{2}$-groups by free abelian groups of rank $>1$ are never cohopfian, and it is probable that no virtually polycyclic group is cohopfian.)

Groups of aspherical geometric 3-manifolds in classes (1), (2), (3) and (6) satisfy the volume condition [157]. Again, this may be verified using the Gromov norm for classes (1) and (2), and class (3) presents 
the most difficulty. (Class (6) can be handled as in Theorem 12.10.) Since $\mathrm{PD}_{3}$-groups in the other classes are not cohopfian they do not satisfy the volume condition.

All $\mathrm{PD}_{3}$-groups in any one of the classes (5), (6), (7) or (8) are commensurable, while the commensurability classes of groups of type (4) correspond to the real quadratic extensions of $\mathbb{Q}$. The commensurability classification of hyperbolic 3-manifolds and 3-manifolds with nontrivial geometric decompositions (corresponding to classes (1), (2) and (3)) is considerably more delicate. See [111; 113]. 


\section{Appendix: Some open questions}

In the following sections we shall present a number of questions on subgroups of $\mathrm{PD}_{3}$-groups, motivated by results conjectured or already established geometrically for 3-manifold groups. The underlying question is whether every $\mathrm{PD}_{3}$-group $G$ is the fundamental group of some aspherical closed 3-manifold. The following questions represent possibly simpler consequences. (If we assume $G$ is coherent and has a finite $K(G, 1)$-complex, as is the case for all 3-manifold groups, a number of these questions have clear answers.)

The corresponding questions for subgroups of open $\mathrm{PD}_{3}$-groups should be considered with these. Any group with an Eilenberg-Mac Lane complex which is a finite 2-complex is the fundamental group of a compact aspherical 4-manifold with boundary, obtained by attaching 1- and 2-handles to $D^{4}$. (Conjecturally such groups are exactly the finitely presentable groups of cohomological dimension 2). On applying the reflection group trick of Davis to the boundary, we see that each such group embeds in a $\mathrm{PD}_{4}$-group [24]. Thus the case considered here is critical.

We assume throughout that $G$ is an orientable $\mathrm{PD}_{3}$-group.

\section{A.1. The group}

If $M$ is a closed 3-manifold then $\pi=\pi_{1}(M)$ has a finite presentation of deficiency 0 . Moreover, $M$ has a Heegaard decomposition as the union of two cubes with handles along a closed surface, and so $\pi$ has dual presentations, in the sense of [146]. If $\pi$ is torsion-free then it is FF, i.e., the augmentation module $\mathbb{Z}$ has a finite free $\mathbb{Z}[\pi]$-resolution, while $\widetilde{K}_{0}(\mathbb{Z}[\pi])=\mathrm{Wh}(\pi)=0$ and $\widetilde{M} \cong \mathbb{R}^{3}$, so $\pi$ is 1 -connected at $\infty$. If $M$ is aspherical then $\beta_{1}^{(2)}(\pi)=0$, and so all the $L^{2}$ Betti numbers of $\pi_{1}(M)$ are 0 [Lu, Theorem 4.1].

If $G$ is a $\mathrm{PD}_{3}$-group then $\operatorname{def}(G) \leqslant 0$, since $\beta_{1}\left(G ; \mathbb{F}_{2}\right)=\beta_{2}\left(G ; \mathbb{F}_{2}\right)$. The augmentation module $\mathbb{Z}$ has a finite projective $\mathbb{Z}[G]$-resolution, so $G$ is almost finitely presentable $\left(\mathrm{FP}_{2}\right)$, and has a 3-dimensional $K(G, 1)$ complex. The $K(G, 1)$-complex is finitely dominated, and hence a Poincaré complex, if and only if $G$ is finitely presentable. It is homotopy equivalent to a finite complex if and only if $G$ is also of type FF.

For each $g \in G$ with infinite conjugacy class, we have $\left[G: C_{G}(\langle g\rangle)\right]=\infty$, so $\operatorname{cd} C_{G}(\langle g\rangle) \leqslant 2$, by the Strebel Theorem. Hence $C_{G}(\langle g\rangle) /\langle g\rangle$ is locally virtually free, by [Bi, Theorem 8.4]. Therefore $G$ must satisfy the Strong Bass Conjecture [30].

An $\mathrm{FP}_{2}$ group $S$ with $H^{2}(S ; \mathbb{Z}[S]) \cong \mathbb{Z}$ is virtually a $\mathrm{PD}_{2}$-group [15].

(1) Is $G$ finitely presentable?

(2) If $G$ is finitely presentable, does it have deficiency 0 ?

(3) Is $G$ of type FF? 
(4) Is $\widetilde{K}_{0}(\mathbb{Z}[G])=0$ ? Is $\mathrm{Wh}(G)=0$ ?

(5) Is $G 1$-connected at $\infty$ ?

(6) Is $K(G, 1)$ homotopy equivalent to a finite complex?

(7) Is $\beta_{1}^{(2)}(G)=0$ ?

(8) If a group $S$ is $\mathrm{FP}_{3}$ and $H^{3}(S ; \mathbb{Z}[S]) \cong \mathbb{Z}$, is $S$ virtually a $\mathrm{PD}_{3}$-group?

If (8) is true, then centres of 2-knot groups are finitely generated.

\section{A.2. Subgroups in general}

Since $G$ has cohomological dimension 3, it has no nontrivial finite subgroups. Any nontrivial element $g$ generates an infinite cyclic subgroup $\langle g\rangle$; it is not known whether there need be any other proper subgroups. If a subgroup $H$ of $G$ has finite index then it is also a $\mathrm{PD}_{3}$-group. The cases when $[G: H]$ is infinite are of more interest, and then either cd $H=2$ or $H$ is free, by the Strebel Theorem. If there is a finitely generated (respectively, $\mathrm{FP}_{2}$ ) subgroup of cohomological dimension 2, there is one such which has one end (i.e., which is indecomposable with respect to free product). A solvable subgroup $S$ of Hirsch length $h(S) \geqslant 2$ must be polycyclic, since either $[G: S]$ is finite or cd $S=2=h(S)$, so $S \cong \mathrm{BS}(1, m)$ for some $m \neq 0$ [48], and no nontrivial Baumslag-Solitar relation holds in $G$. (In particular, abelian subgroups of rank $>1$ are finitely generated.)

Three-manifold groups are coherent: finitely generated subgroups are finitely presentable. In fact, something stronger is true: if $H$ is a finitely generated subgroup, it is the fundamental group of a compact 3-manifold (possibly with boundary), by Scott's Core Theorem [123]. If $\pi$ is the fundamental group of a graph manifold then the group ring $\mathbb{Z}[\pi]$ is coherent as a ring. (The corresponding result for lattices in $\operatorname{PSL}(2, \mathbb{C})$ is apparently not known.)

If $G$ is a $\mathrm{PD}_{3}$-group with a one-ended $\mathrm{FP}_{2}$ subgroup $H$, then $H$ is the ambient group of a $\mathrm{PD}_{3}$-pair of groups, by the Algebraic Core Theorem. Hence $\chi(H) \leqslant 0$, and so $H$ cannot be a product $F \times F$ with $F$ a noncyclic free group [101]. As such groups $F \times F$ have finitely generated subgroups which are not finitely related, this may be regarded as weak evidence for coherence. (On the other hand, every surface group $\sigma$ with $\chi(\sigma)<0$ has such a subgroup $F$, and so $F \times F$ is a subgroup of $\sigma \times \sigma$. Thus $\mathrm{PD}_{n}$-groups with $n \geqslant 4$ need not be coherent.)

Let $M$ be an aspherical closed orientable 3-manifold. Then $M$ is Haken, Seifert fibred or hyperbolic, by the Geometrization Theorem. With [79] it follows that if $\pi_{1}(M)$ is infinite then it has a $\mathrm{PD}_{2}$-subgroup. A transversality argument implies that every element of $H_{2}(M ; \mathbb{Z}) \cong H^{1}(M ; \mathbb{Z}) \cong\left[M ; S^{1}\right]$ is represented by an embedded submanifold. If $M$ is aspherical, it follows that $H_{2}\left(\pi_{1}(M)\right.$; $\left.\mathbb{Z}\right)$ is generated by elements represented by surface subgroups of $\pi_{1}(M)$.

If $M$ is a closed aspherical 3-manifold which is not a graph manifold then $M$ has a finite covering space which fibres over the circle [1;117]. Indecomposable finitely generated subgroups of infinite index in such groups are (finitely presentable) virtually semidirect products $F \rtimes \mathbb{Z}$, with $F$ a free group. Every such group $H$ is an HNN extension with finitely generated free base, and one of the associated subgroups a free factor of the base [38]. In particular, $H$ is finitely presentable and has a finite 2-dimensional $K(H, 1)$-complex. 
If $G / G^{\prime}$ is infinite then $G$ is an HNN extension with finitely generated base and associated subgroups, by the Bieri-Strebel Theorem, and so has a finitely generated subgroup of cohomological dimension 2. If, moreover, $G$ is almost coherent then it has a $\mathrm{PD}_{2}$-subgroup, by the Algebraic Core Theorem.

(9) Is there a noncyclic proper subgroup? If so, is there one of cohomological dimension 2 ? and finitely generated?

(10) Is there a subgroup which is a surface group?

(11) Is every element of $H_{2}(G ; \mathbb{Z})$ represented by a $\mathrm{PD}_{2}$-subgroup?

(12) Is $G$ (almost) coherent?

(13) Is $\mathbb{Z}[G]$ coherent as a ring?

(14) Does every (finitely presentable) subgroup of cohomological dimension 2 have a (finite) 2-dimensional Eilenberg-Mac Lane complex?

(15) Let $H$ be a finitely generated subgroup with one end and of infinite index in $G$. Does $H$ have infinite abelianization? Does $H$ contain a surface group?

(16) Is every indecomposable finitely generated subgroup of infinite index in $G$ virtually a semidirect product $F \rtimes \mathbb{Z}$, with $F$ free?

\section{A.3. Normal subgroups}

If $N$ is an $\mathrm{FP}_{2}$ ascendant subgroup of $G$ and $\operatorname{cd} N=2$, then $N$ is a $\mathrm{PD}_{2}$-group and $G$ is the fundamental group of a 3-manifold finitely covered by a surface bundle. If $\operatorname{cd} N=1$ then $N \cong \mathbb{Z}$, and either $G$ is polycyclic, or $N$ is normal in $G$ and $G$ is the fundamental group of a Seifert fibred 3-manifold. (See Chapters 8 and 9.) It is easy to find examples among normal subgroups of 3-manifold groups to show that finite generation of $N$ is necessary for these results.

If $N$ is finitely generated and normal and if $[G: N]=\infty$, then $E(G / N)=H^{1}(G / N ; \mathbb{Z}[G / N])$ is isomorphic to $H^{1}(G ; \mathbb{Z}[G / N])$, and hence also to $H_{2}(G ; \mathbb{Z}[G / N]) \cong H_{2}(N ; \mathbb{Z})$, by Poincaré duality. If $G / N$ has two ends, then after passing to a subgroup of finite index, we may assume that $G / N \cong \mathbb{Z}$. It then follows from the PD Extension Theorem that $N$ is again a $\mathrm{PD}_{2}$-group [Hi, Theorem 1.19].

(17) Is there a simple $\mathrm{PD}_{3}$-group?

(18) Is $v \beta(G)>0$ ?

(19) Must a finitely generated normal subgroup $N$ be finitely presentable?

(20) Let $U$ be an $\mathrm{FP}_{2}$ subgroup such that $\operatorname{Core}_{G}(U)=\bigcap_{g \in G} g U g^{-1}$ is not finitely generated. Is $U$ commensurable with a subgroup $V$ such that $\left[G: N_{G}(V)\right]<\infty$ ? Is this at least so if $U$ is indecomposable and $G$ is $U$-residually finite?

(21) If $G^{\prime}$ is free, is $G$ a semidirect product $K \rtimes \mathbb{Z}$ with $K$ a $\mathrm{PD}_{2}$-group?

\section{A.4. Centralizers, normalizers and commensurators}

Every strictly increasing sequence of centralizers $C_{0}<C_{1}<\cdots<C_{n}=G$ in a $\mathrm{PD}_{3}$-group $G$ has length $n$ at most 4 . If $G$ has an abelian subgroup $A$ which is not finitely generated, then $C_{G}(A)$ is abelian, and 
$1<C_{G}(A)<G$ is a maximal sequence of centralizers. (On the other hand, the chain of centralizers $C_{B}\left(x^{2^{k}}\right)$ with $k \geqslant 0$ in $B=\mathrm{BS}(2,3)=\left\langle t, x \mid t x^{2} t^{-1}=x^{3}\right\rangle$ is infinite, and hence the $\mathrm{PD}_{4}$-group obtained from $B$ by the Davis construction does not have max-c [97].)

If $h \in G$, then $C_{G}(h)$ is finitely generated if and only if $h$ is not infinitely divisible. An element $g$ is a root of $h$ if $h=g^{n}$ for some $n$. All roots of $h$ are in $C_{G}(h)$. If every abelian subgroup of $G$ is finitely generated then $C_{G}(h)$ is finitely generated, for all $h \in G$. It then follows that every centralizer is either $\mathbb{Z}$, finitely presentable and of cohomological dimension 2 or of finite index in $G$. (Applying the Davis construction to $\mathrm{BS}(1,2)=\mathbb{Z} *_{2}$ gives a $\mathrm{PD}_{4}$-group with an abelian subgroup which is not finitely generated [108].)

If $x$ is a nontrivial element of $G$ then $\left[N_{G}(\langle x\rangle): C_{G}(x)\right] \leqslant 2$ (since $\langle x\rangle \cong \mathbb{Z}$ ). If $H$ is an $\mathrm{FP}_{2}$ subgroup which is a nontrivial free product but is not free, then $\left[N_{G}(H): H\right]<\infty$ and $C_{G}(H)=1$, by Theorem 9.7. If $F$ is a finitely generated nonabelian free subgroup of $G$, then $N_{G}(F)$ is finitely generated and $N_{G}(F) / F$ is finite or virtually $\mathbb{Z}$, by Theorem 9.8 .

If $G$ has a subgroup $H$ which is $F P_{2}$ and has one end with $\chi(H)=0$ (respectively, $\chi(H)<0$ ), then either $H$ is commensurable with its own normalizer in $G$ or $G$ is virtually the group of a surface bundle, by Corollary 9.12.1. Either $\left[\operatorname{Comm}_{G}(H): H\right]<\infty$ or $H$ is commensurable with a subgroup $K$ such that $\left[G: N_{G}(K)\right]<\infty$. In the later case $H$ is a $\mathrm{PD}_{2}$-group and $\left[G: \operatorname{Comm}_{G}(H)\right]<\infty$.

If $x \neq 1$ then $\operatorname{Comm}_{G}(\langle x\rangle)=\bigcup_{n \geq 1} N_{G}\left(\left\langle x^{n !}\right\rangle\right)$, since no nontrivial Baumslag-Solitar relation holds in $G$. Since the chain of centralizers $C_{G}\left(\left\langle x^{n !}\right\rangle\right)$ is increasing and $\left[N_{G}\left(\left\langle x^{k}\right\rangle\right): C_{G}\left(\left\langle x^{k}\right\rangle\right)\right] \leqslant 2$ for any $k$, it follows that $\operatorname{Comm}_{G}(\langle x\rangle)=N_{G}\left(\left\langle x^{n !}\right\rangle\right)$ for some $n \geqslant 1$.

(22) Is every abelian subgroup finitely generated?

(23) If $G$ is not virtually abelian and $H$ is an $\mathrm{FP}_{2}$ subgroup such that $N_{G}(H)=H$, is $\left[\operatorname{Comm}_{G}(H): H\right]$ finite?

(24) Is there a $\mathrm{PD}_{3}$-group $G$ with a finitely generated subgroup $U$ such that $[G: U]=\infty, N_{G}(U)=U$ and $\operatorname{Core}_{G}(U)=\bigcap_{g \in G} g U g^{-1} \neq 1$ ?

\section{A.5. The derived series and perfect subgroups}

Let $G^{(\omega)}=\bigcap G^{(n)}$ be the intersection of the terms of the derived series for $G$. If $G^{(\omega)}=G^{(n)}$ for some finite $n$ then $n \leqslant 3$, and either $G / G^{(\omega)}$ is a finite solvable group with cohomological period dividing 4 (and $G^{(\omega)}$ is a perfect $\mathrm{PD}_{3}$-group), or it has two ends and is $\mathbb{Z}, \mathbb{Z} \oplus \mathbb{Z} / 2 \mathbb{Z}$ or $D_{\infty}$, or it has one end and is a solvable $\mathrm{PD}_{3}$-group. There is a similar result for the lower central series. If $G$ is orientable and $G_{[\omega]}=G_{[n]}$ for some finite $n$, then $n \leqslant 3$, and $G / G_{[\omega]}$ is finite, $\mathbb{Z}$ or a nilpotent $\mathrm{PD}_{3}$-group. (See Chapter 11.) If $G / G^{(\omega)}$ is infinite and $v \beta(G)=0$, then $G / G^{(\omega)}$ is a finitely generated residually finite-solvable group with one or infinitely many ends, and $v \beta(G ; p)=\infty$ for some prime $p$.

Let $M$ be the (aspherical) 3-manifold obtained by 0 -framed surgery on a nontrivial knot $K$ with Alexander polynomial $\Delta_{K} \doteq 1$, and let $G=\pi_{1}(M)$. Then $G^{\prime}$ is a perfect normal subgroup which is not finitely generated. (In this case $G_{[\omega]}=G^{(\omega)}=G^{\prime}$, and $G / G^{(\omega)} \cong \mathbb{Z}$.) Replacing a suitable solid torus in $R P^{3} \sharp R P^{3}$ by the exterior of such a knot $K$ gives an example with $G / G^{(\omega)} \cong D_{\infty}$.

Let $\kappa$ be a perfect normal subgroup of the fundamental group $\pi$ of a $\mathrm{PD}_{3}$-complex $X$. Then $\rho=\pi / \kappa$ is $\mathrm{FP}_{2}$, since $\pi$ is $\mathrm{FP}_{2}$ and $H_{1}(\kappa ; \mathbb{Z})=0$. The arguments of [22] give $\rho \cong\left(*_{i=1}^{r} G_{i}\right) * V$, where each factor $G_{i}$ has one end and $V$ is virtually free. Moreover, if $\rho$ is infinite and has a nontrivial finite normal 
subgroup then $\rho$ has two ends. (However, the further analysis of [68] does not apply, since there is no analogue of the Splitting Theorem of Turaev.) We also have $H_{2}(\kappa ; \mathbb{Z}) \cong H^{1}(\rho ; \mathbb{Z}[\rho])$ as an abelian group. In particular, if $\kappa$ is acyclic then $\rho$ has one end.

The intersection $P=\bigcap G^{(\alpha)}$ of the terms of the transfinite derived series for $G$ is the maximal perfect subgroup of $G$, and is normal in $G$. The quotient $G / P$ is $\mathrm{FP}_{2}$. If $P \neq 1$ and $[G: P]$ is infinite then cd $P=2$, but $P$ cannot be $\mathrm{FP}_{2}$, for otherwise it would be a surface group, by Theorem 8.9. Note that $P \subseteq G^{(\omega)}$, and if $\operatorname{cd} P=2$ then $\operatorname{cd} G^{(\omega)}=2$ also. If $[G: P]$ is infinite and $\zeta G \neq 1$ then $P=1$.

If $G$ is a $\mathrm{PD}_{3}$-group and $H$ is a nontrivial $\mathrm{FP}_{2}$ subgroup such that $H^{1}(H ; \mathbb{Z})=0$, then $[G: H]$ is finite. (For if $[G: H]=\infty$ and $J$ is an indecomposable factor of $H$, then $J$ would be the ambient group of a $\mathrm{PD}_{3}$-pair of groups, by the Algebraic Core Theorem. But then $\chi(J) \leqslant 0$ and so $H^{1}(H ; \mathbb{Z}) \neq 0$.) See [74] for 3-manifold groups.

(25) Can a nontrivial finitely generated normal subgroup of infinite index be perfect? acyclic?

(26) If $H$ is a finitely generated residually solvable group with infinitely many ends, is $v \beta(H)>0$ ?

(27) If $P=1$, is $G$ residually solvable (i.e., is $G^{(\omega)}=1$ also)?

\section{A.6. The Tits alternative}

Let $N$ be the maximal normal restrained subgroup of $G$. Then $N$ contains the maximal elementary amenable normal subgroup of $G$. If $N$ is nontrivial then either $N \cong \mathbb{Z}, \operatorname{cd} N=2$ or $N=G$. If $N$ is abelian then $N \leqslant \sqrt{G}$. If $\mathrm{cd} N=2$ then $N$ cannot be $\mathrm{FP}_{2}$, for otherwise it would be a $\mathrm{PD}_{2}$-group and $G$ would be virtually the group of a surface bundle, by Theorem 8.9. Since $N$ is restrained this would imply that $G$ is polycyclic, and so $N=G$. Similarly, if $N=G$ and $\beta_{1}(G) \geqslant 2$ then there is an epimorphism $\phi: G \rightarrow \mathbb{Z}$ with finitely generated kernel [11]. Hence $\operatorname{Ker}(\phi)$ is a surface group, and so $G$ is polycyclic. If $G$ is almost coherent and $N$ is neither $G$ nor 1 , then $N \cong \mathbb{Z}$.

If $G$ is an almost coherent $\mathrm{PD}_{3}$-group which is not virtually properly locally cyclic, then every finitely generated subgroup of $G$ satisfies the Tits alternative. (See Chapter 11.) Solvable subgroups are abelian or polycyclic.

(28) Is $N$ the maximal elementary amenable normal subgroup?

(29) If $H$ is a finitely generated subgroup which has no nonabelian free subgroup, must it be polycyclic?

(30) If $G$ has subexponential growth, is it virtually nilpotent?

(31) Is $G$ either solvable or large?

\section{A.7. Atoroidal groups}

Two-generator subgroups of atoroidal almost coherent $\mathrm{PD}_{3}$-groups are either free or of finite index, by [5] together with the Algebraic Core Theorem of [81]. 3-Manifolds with atoroidal fundamental group are hyperbolic, by the Geometrization Theorem. Every closed hyperbolic 3-manifold has a finite covering space which fibres over the circle $[1 ; 117]$.

If $G$ is atoroidal and acts geometrically on a locally compact CAT( 0$)$ space, then it is Gromov hyperbolic [82]. It then has boundary $S^{2}$ [7].

(32) Is every atoroidal $\mathrm{PD}_{3}$-group Gromov hyperbolic? 
(33) Does every atoroidal $\mathrm{PD}_{3}$-group have a boundary as in [6]?

(34) The Cannon Conjecture: Is every Gromov hyperbolic $\mathrm{PD}_{3}$-group isomorphic to a lattice in PSL(2, $\left.\mathbb{C}\right)$ ?

(35) Does every atoroidal $\mathrm{PD}_{3}$-group have a nontrivial finitely generated subnormal subgroup of infinite index?

\section{A.8. Splitting}

The central role played by incompressible surfaces in the geometric study of Haken 3-manifolds suggests strongly the importance of splitting theorems for $\mathrm{PD}_{3}$-groups. This issue was raised in [142], the first paper on $\mathrm{PD}_{3}$-groups, and considered in detail by Kropholler and Roller. Every $\mathrm{PD}_{3}$-group has max-c, and so has a JSJ decomposition. (See Chapter 10, and also [26].)

If $G$ is restrained and $G / G^{\prime}$ is infinite, then $G$ is an ascending HNN extension with finitely generated base. If $G$ is an ascending $\mathrm{HNN}$ extension with $\mathrm{FP}_{2}$ base $H$, then $H$ is a $\mathrm{PD}_{2}$-group and is normal in $G$, and so $G$ is the group of a surface bundle, by Theorems 11.1 and 8.9 , respectively.

If $G$ is residually finite and has a subgroup $H \cong \mathbb{Z}^{2}$, then it is virtually split over a subgroup commensurate with $H$ [98]. Moreover, either $G$ is polycyclic or it has a subgroup of finite index which maps onto a nonabelian free group, by Theorem 11.19 .

(36) If $G$ is a nontrivial free product with amalgamation or $\mathrm{HNN}$ extension, does it split over a $\mathrm{PD}_{2}$ group?

(37) If $G$ is a nontrivial free product with amalgamation, is $v \beta(G)>0$ ?

(38) Can $G$ be a properly ascending $\mathrm{HNN}$ extension (with base finitely generated, but not $\mathrm{FP}_{2}$ )?

(39) If a subgroup $H$ is a $\mathrm{PD}_{2}$-group and $\operatorname{Comm}_{G}(H)=H$, does $G$ have a subgroup of finite index which splits over $H$ ? In particular, is this so if $H \cong \mathbb{Z}^{2}$ ?

(40) Is there a bound for splittings of $\mathrm{PD}_{n}$-pairs of groups along $\mathrm{PD}_{n-1}$-pairs which extends [26]?

(41) Suppose that $G$ is an HNN extension with stable letter $t$, base $H$ and associated subgroup $F \subset H$. Is $\mu(G)=\bigcap t^{k} F t^{-k}$ finitely generated? (See [80] for a related result on knot groups, and also [131].)

\section{A.9. Residual finiteness, hopficity, cohopficity}

The groups of 3-manifolds are residually finite, and so are hopfian [AFW]. Since BS(2, 3) is non-hopfian [MKS, page 260], and embeds in a $\mathrm{PD}_{4}$-group by the Davis construction, there are $\mathrm{PD}_{4}$-groups which are not residually finite [108]. (No nontrivial Baumslag-Solitar relation holds in any $\mathrm{PD}_{3}$-group $G$. See Theorem 10.12.)

Many 3-manifold groups satisfy the stronger RFRS (residually finite and rationally solvable) condition: there is a descending series of finite-index normal subgroups $R_{n}$ such that $\bigcap R_{n}=1$ and such that the natural epimorphism from $R_{n}$ to $R_{n} / R_{n+1}$ factors through the quotient of $R_{n} / R_{n}^{\prime}$ by its torsion subgroup. Every 3-manifold with virtually RFRS fundamental group is virtually fibred [1]. This has a purely algebraic analogue. A finitely generated virtually RFRS group $S$ is virtually a semidirect product $H \rtimes \mathbb{Z}$ with $H$ finitely generated if and only if $\beta_{1}^{(2)}(S)=0$ [84]. The $L^{2}$ Betti numbers of 3-manifold groups are all 0 . If $G$ is a $\mathrm{PD}_{3}$-group then it follows from the $L^{2}$-Approximation Theorem that $\beta_{1}^{(2)}(G)=0$ if and only if the condition of (46) below holds for $G$ and all of its subgroups of finite index. 
A 3-manifold group satisfies the volume condition if and only if it is not solvable and is not virtually a product [155; 157]. In particular, such 3-manifold groups are cohopfian, i.e., injective endomorphisms are automorphisms. The volume condition is a property of commensurability classes; this is not so for cohopficity.

If $G$ is a finitely presentable, residually finite group then $\beta_{1}^{(2)}(G)=\lim \frac{\beta_{1}(H)}{[G: H]}$, where the limit is taken over the directed system of subgroups $H$ of finite index, by the $L^{2}$ Aproximation Theorem. Hence $\beta_{1}^{(2)}(G)=0$ if and only if (46) holds for $G$ and all of its subgroups of finite index.

(42) Does $G$ have a proper subgroup of finite index?

(43) Is $G$ residually finite?

(44) Are atoroidal $\mathrm{PD}_{3}$-groups virtually RFRS?

(45) Suppose that $\widehat{G}$ is a pro- $p \mathrm{PD}_{3}$-group. Is $v \beta(G)>0$ ?

(46) If $\beta_{1}(G)>0$ is there a subgroup $H$ of finite index such that $\beta_{1}(H) \leqslant \frac{1}{2}[G: H] \beta_{1}(G)$ ?

(47) Do all $\mathrm{PD}_{3}$-groups other than those which are solvable or are virtually products satisfy the volume condition?

\section{A.10. Other questions}

We conclude with some related questions. (In the first six of these questions $P$ is an indecomposable $\mathrm{PD}_{3}$-space.)

(48) Is $P$ homotopy equivalent to a standard $\mathrm{PD}_{3}$-complex?

(49) Is $P$ homotopy equivalent to a 3-manifold if its orientable double cover $P^{+}$is homotopy equivalent to a 3-manifold?

(50) Is $P \times S^{1}$ or $P \times S^{1} \times S^{1}$ homotopy equivalent to a closed manifold?

(51) Clarify the connection between $k_{1}(P)$ and $c_{P *}[P]$ when $\pi_{1}(P)$ is infinite.

(52) If $\pi_{1}(P) \cong \pi \mathcal{G}$, where $(\mathcal{G}, \Gamma)$ is a graph of groups with vertex groups one-ended and edge groups of order 2 , must each vertex of $\Gamma$ have even valence?

(53) Does $P$ have a 3-manifold 1-skeleton, i.e., is $P \simeq H \cup_{\partial} Y$, where $H$ is a cube with handles and $(Y, \partial H)$ is a 1-connected $\mathrm{PD}_{3}$-pair?

(54) Is $\left(D_{2 m} \times \mathbb{Z} / 3 \mathbb{Z}\right) * \mathbb{Z} / 6 \mathbb{Z} T_{1}^{*}$ the group of a $\mathrm{PD}_{3}$-complex, for some $m \geqslant 5$ ?

(55) Find an explicit example of a free action of a generalized quaternionic group $Q(8 a, b, 1)$ (with $a, b>1$ and $(a, b)=1)$ on an homology 3 -sphere.

(56) Can the Classification and Realization Theorems be extended to $\mathrm{PD}_{3}$-pairs with spherical boundary components?

(57) Let $(G, \Omega)$ be a $\mathrm{PD}_{3}$-pair of groups. Suppose that $\theta \in H^{1}(G ; \mathbb{Z})$ has nontrivial restriction to each boundary component. If $\operatorname{Ker}(\theta)$ is locally free, must it be free? (See [42].)

(58) Does every finite group with cohomological period 4 have a balanced presentation?

(59) Let $G$ be a group with infinite subgroups $N<U$ of infinite index, such that $N$ is normal in $G$ and $U$ is finitely generated. Is $\beta_{1}^{(2)}(G)=0$ ? 
(60) Is there an algebraic analogue of the reflection group trick which may be used to embed FP groups of cohomological dimension $k$ in $\mathrm{PD}_{2 k}$-groups?

(61) Are there any indecomposable $\mathrm{PD}_{3}$-complexes other than $R P^{2} \times S^{1}$ whose groups have an element of order 2 with infinite centralizer?

(62) Suppose that $G$ is $\mathrm{FP}_{2}$, has one end and cd $G=2$. Is $G$ the ambient group of at most finitely many $\mathrm{PD}_{3}$-pairs of groups?

(63) Suppose that $G$ is an atoroidal $\mathrm{PD}_{3}$-group. Is the outer automorphism group Out $(G)$ finite?

(64) Is there a useful notion of open $\mathrm{PD}_{3}$-complex which includes all infinite covering spaces of $\mathrm{PD}_{3}$ complexes?

Every $\mathrm{PD}_{3}$-complex is homotopy equivalent to one of the form $Y \cup e^{3}$, where $Y$ is cohomologically 2-dimensional. Thus (48) is related to Wall's $\mathcal{D}(2)$-problem. (See [Jo].) If it holds, then $\pi$ has deficiency $\geqslant 0$, as we may assume that $X$ has one 0-cell, and $\chi(X)=0$. Hence (48) implies (2).

One way to prove (52) might be to extend the Realization Theorem to show that $\left(G_{v},\left\{G_{e}: o(e)=\right.\right.$ $v$ or $t(e)=v, o(e) \neq t(e)\})$ is the fundamental group system of a $\mathrm{PD}_{3}$-pair (with all boundary components $R P^{2}$ ), for each vertex $v \in V(\Gamma)$.

Since there are $\mathrm{PD}_{3}$-complexes which are not homotopy equivalent to 3-manifolds, and thus which do not have homotopy Heegaard decompositions, we cannot expect that $(Y, \partial H)$ is also homotopy equivalent to a cube with handles in (53).

Reeves, Scott and Swarup have given an algebraic analogue of Johannson's Deformation Theorem [119]. In the 3-manifold case this theorem implies that aspherical 3-manifolds with incompressible boundary are determined up to finite ambiguity by their fundamental groups, and we may expect the analogous result to hold for $\mathrm{PD}_{3}$-pairs of groups. This suggested question (63) above. It is true in the atoroidal, aspherical case: if $(G, \Omega)$ is an atoroidal $\mathrm{PD}_{3}$-pair of groups and $(Q, \partial Q)$ is a $\mathrm{PD}_{3}$-pair with $\pi_{1}(Q) \cong G$ and aspherical boundary components then $Q$ is aspherical and the peripheral system of $(Q, \partial Q)$ is isomorphic to $(G, \Omega)$ [71, Theorem 28]. Note, however, that the $\mathrm{PD}_{3}$-pairs of groups corresponding to the exteriors of the reef and granny knots shows that the ambient group need not determine the pair. 


\section{References}

\section{Books}

[AFW] M. Aschenbrenner, S. Friedl, and H. Wilton, 3-manifold groups, European Mathematical Society, Zürich, 2015.

[AM] A. Adem and R. J. Milgram, Cohomology of finite groups, 2nd ed., Grundlehren der Mathematischen Wissenschaften 309, Springer, 2004.

[Bi] R. Bieri, "Homological dimension of discrete groups", Queen Mary College Mathematics Notes, Queen Mary College, London, 1976.

[Br] K. S. Brown, Cohomology of groups, Graduate Texts in Mathematics 87, Springer, 1982.

[CR] C. W. Curtis and I. Reiner, Representation theory of finite groups and associative algebras, Pure and Applied Mathematics 11, Interscience, 1962.

[DD] W. Dicks and M. J. Dunwoody, Groups acting on graphs, Cambridge Studies in Advanced Mathematics 17, Cambridge University Press, Cambridge, 1989.

[FM] B. Farb and D. Margalit, A primer of mapping class groups, Princeton Univ. Press, Princeton, NJ, 2015.

[FQ] M. H. Freedman and F. Quinn, Topology of 4-manifolds, Princeton Mathematical Series 39, Princeton University Press, 1990.

[Ge] R. Geoghegan, Topological methods in group theory, Graduate Texts in Mathematics 243, Springer, 2008.

[GW] F. González-Acuña and W. C. Whitten, "Imbeddings of three-manifold groups”, Mem. Amer. Math. Soc. 99:474 (1992), viii+55.

[Hi] J. A. Hillman, Four-manifolds, geometries and knots, Geometry \& Topology Monographs 5, Geometry \& Topology Publications, Coventry, 2002.

[Jo] F. E. A. Johnson, Stable modules and the D(2)-problem, London Mathematical Society Lecture Note Series 301, Cambridge University Press, 2003.

[JS] W. H. Jaco and P. B. Shalen, "Seifert fibered spaces in 3-manifolds", Mem. Amer. Math. Soc. 21:220 (1979), viii+192.

[Lu] W. Lück, $L^{2}$-invariants: theory and applications to geometry and $K$-theory, Ergebnisse der Mathematik und ihrer Grenzgebiete. 3. Folge. A Series of Modern Surveys in Mathematics [Results in Mathematics and Related Areas. 3rd Series. A Series of Modern Surveys in Mathematics] 44, Springer, 2002.

[McC] J. McCleary, User's guide to spectral sequences, Mathematics Lecture Series 12, Publish or Perish, Wilmington, DE, 1985.

[MKS] W. Magnus, A. Karrass, and D. Solitar, Combinatorial group theory: Presentations of groups in terms of generators and relations, Interscience, New York, 1966. Reprinted Dover, New York, 1966.

[Ol] A. Y. Olshanskii, Геометрия определяющих соотношений в группах, Nauka, Moscow, 1989.

[Ra] A. Ranicki, Algebraic and geometric surgery, The Clarendon Press, Oxford, 2002. Oxford Science Publications.

[Ro] D. J. S. Robinson, A course in the theory of groups, 2nd ed., Graduate Texts in Mathematics 80, Springer, 1996.

[Se] J.-P. Serre, Trees, Springer, 2003.

\section{Articles}

[1] I. Agol, "The virtual Haken conjecture", Doc. Math. 18 (2013), 1045-1087.

[2] D. Allcock, "Spherical space forms revisited", Trans. Amer. Math. Soc. 370:8 (2018), 5561-5582.

[3] H. Bass, "Projective modules over free groups are free", J. Algebra 1 (1964), 367-373.

[4] H. J. Baues and B. Bleile, "Poincaré duality complexes in dimension four", Algebr. Geom. Topol. 8:4 (2008), 23552389.

[5] G. Baumslag and P. B. Shalen, "Groups whose three-generator subgroups are free", Bull. Austral. Math. Soc. 40:2 (1989), 163-174. 
[6] M. Bestvina, "Local homology properties of boundaries of groups", Michigan Math. J. 43:1 (1996), 123-139.

[7] M. Bestvina and G. Mess, "The boundary of negatively curved groups", J. Amer. Math. Soc. 4:3 (1991), 469-481.

[8] R. Bieri and B. Eckmann, "Relative homology and Poincaré duality for group pairs", J. Pure Appl. Algebra 13:3 (1978), 277-319.

[9] R. Bieri and J. A. Hillman, "Subnormal subgroups of 3-dimensional Poincaré duality groups", Math. Z. 206:1 (1991), 67-69.

[10] R. Bieri and R. Strebel, "Almost finitely presented soluble groups", Comment. Math. Helv. 53:2 (1978), 258-278.

[11] R. Bieri, W. D. Neumann, and R. Strebel, "A geometric invariant of discrete groups”, Invent. Math. 90:3 (1987), 451477.

[12] B. Bleile, "Poincaré duality pairs of dimension three", Forum Math. 22:2 (2010), 277-301.

[13] B. Bleile, I. Bokor, and J. A. Hillman, "Poincaré duality complexes with highly connected universal cover", Algebr. Geom. Topol. 18:7 (2018), 3749-3788.

[14] M. Boileau and S. Boyer, "On the Tits alternative for P D(3) groups", Ann. Fac. Sci. Toulouse Math. (6) 28:3 (2019), 397-415.

[15] B. H. Bowditch, "Planar groups and the Seifert conjecture", J. Reine Angew. Math. 576 (2004), 11-62.

[16] K. S. Brown and R. Geoghegan, "Cohomology with free coefficients of the fundamental group of a graph of groups", Comment. Math. Helv. 60:1 (1985), 31-45.

[17] J. O. Button, "Strong Tits alternatives for compact 3-manifolds with boundary", J. Pure Appl. Algebra 191:1-2 (2004), 89-98.

[18] M. Carette, "The automorphism group of accessible groups", J. Lond. Math. Soc. (2) 84:3 (2011), 731-748.

[19] A. Casson and D. Jungreis, "Convergence groups and Seifert fibered 3-manifolds", Invent. Math. 118:3 (1994), 441456.

[20] F. Castel, "Centralisateurs d'éléments dans les PD(3)-paires”, Comment. Math. Helv. 82:3 (2007), 499-517.

[21] I. M. Chiswell, "Exact sequences associated with a graph of groups", J. Pure Appl. Algebra 8:1 (1976), 63-74.

[22] J. Crisp, "The decomposition of 3-dimensional Poincaré complexes”, Comment. Math. Helv. 75:2 (2000), 232-246.

[23] J. Crisp, "An algebraic loop theorem and the decomposition of PD ${ }^{3}$-pairs", Bull. Lond. Math. Soc. 39:1 (2007), 46-52.

[24] M. W. Davis, "Poincaré duality groups", pp. 167-193 in Surveys on surgery theory, vol. 1, Ann. of Math. Stud. 145, Princeton Univ. Press, 2000.

[25] J. F. Davis and R. J. Milgram, A survey of the spherical space form problem, Mathematical Reports 2, Harwood, Chur, Switzerland, 1985.

[26] M. J. Dunwoody, "Bounding the decomposition of a Poincaré duality group", Bull. London Math. Soc. 21:5 (1989), 466-468.

[27] M. J. Dunwoody and E. L. Swenson, "The algebraic torus theorem”, Invent. Math. 140:3 (2000), 605-637.

[28] W. G. Dwyer, "Vanishing homology over nilpotent groups", Proc. Amer. Math. Soc. 49 (1975), 8-12.

[29] E. Dyer and A. T. Vasquez, "Some properties of two-dimensional Poincaré duality groups", pp. 45-54 in Algebra, Topology, and category theory (a collection of papers in honor of Samuel Eilenberg), 1976.

[30] B. Eckmann, "Cyclic homology of groups and the Bass conjecture", Comment. Math. Helv. 61:2 (1986), 193-202.

[31] B. Eckmann and P. Linnell, "Poincaré duality groups of dimension two, II", Comment. Math. Helv. 58:1 (1983), 111114.

[32] B. Eckmann and H. Müller, "Poincaré duality groups of dimension two", Comment. Math. Helv. 55:4 (1980), 510-520.

[33] B. Eckmann and H. Müller, "Plane motion goups and virtual Poincaré duality of dimension two", Invent. Math. 69:2 (1982), 293-310. 
[34] A. L. Edmonds, "Deformation of maps to branched coverings in dimension two", Ann. of Math. (2) 110:1 (1979), $113-125$.

[35] H. S. Elkalla, "Subnormal subgroups in 3-manifold groups", J. London Math. Soc. (2) 30:2 (1984), 342-360.

[36] D. B. A. Epstein, "Projective planes in 3-manifolds", Proc. London Math. Soc. (3) 11 (1961), 469-484.

[37] B. Evans and W. Jaco, "Varieties of groups and three-manifolds", Topology 12 (1973), 83-97.

[38] M. Feighn and M. Handel, "Mapping tori of free group automorphisms are coherent", Ann. of Math. (2) 149:3 (1999), 1061-1077.

[39] S. Ferry, W. Lück, and S. Weinberger, “On the stable Cannon Conjecture”, J. Topol. 12:3 (2019), 799-832.

[40] R. H. Fox, "Free differential calculus. I. Derivation in the free group ring", Ann. of Math. (2) 57 (1953), 547-560.

[41] M. H. Freedman, "Automorphisms of circle bundles over surfaces", pp. 212-214 in Geometric topology (Park City, UT, 1974), . Lecture Notes in Math. 438, 1975.

[42] B. Freedman and M. H. Freedman, "Kneser-Haken finiteness for bounded 3-manifolds locally free groups, and cyclic covers", Topology 37:1 (1998), 133-147.

[43] M. Freedman, R. Hain, and P. Teichner, "Betti number estimates for nilpotent groups", pp. 413-434 in Fields Medallists' lectures, World Sci. Ser. 20th Century Math. 5, World Scientific, River Edge, NJ, 1997.

[44] D. Gabai, "Convergence groups are Fuchsian groups", Ann. of Math. (2) 136:3 (1992), 447-510.

[45] S. Gadgil, “The pq-condition for 3-manifold groups", Proc. Amer. Math. Soc. 129:6 (2001), 1873-1875.

[46] S. Gadgil, "Degree-one maps, surgery and four-manifolds", Bull. Lond. Math. Soc. 39:3 (2007), 419-424.

[47] N. D. Gilbert, "Presentations of the automorphism group of a free product", Proc. London Math. Soc. (3) 54:1 (1987), $115-140$.

[48] D. Gildenhuys, "Classification of soluble groups of cohomological dimension two", Math. Z. 166:1 (1979), 21-25.

[49] D. Gildenhuys and R. Strebel, "On the cohomological dimension of soluble groups”, Canad. Math. Bull. 24:4 (1981), 385-392.

[50] F. González-Acuña and W. Whitten, “Co-Hopficity of 3-manifold groups”, Topology Appl. 56:1 (1994), 87-97.

[51] F. González-Acuña, R. Litherland, and W. Whitten, "Co-Hopficity of Seifert-bundle groups", Trans. Amer. Math. Soc. 341:1 (1994), 143-155.

[52] I. Hambleton and I. Madsen, "Actions of finite groups on $\mathbf{R}^{n+k}$ with fixed set $\mathbf{R}^{k}$ ", Canad. J. Math. 38:4 (1986), 781-860.

[53] D. Handel, "On products in the cohomology of the dihedral groups", Tohoku Math. J. (2) 45:1 (1993), 13-42.

[54] W. Heil, "Normalizers of incompressible surfaces in 3-manifolds", Glasnik Mat. Ser. III 16(36):1 (1981), 145-150.

[55] W. Heil and J. Rakovec, "Surface groups in 3-manifold groups", pp. 101-133 in Algebraic and differential topologyglobal differential geometry, Teubner-Texte Math. 70, Teubner, Leipzig, 1984.

[56] J. Hempel and W. Jaco, "Fundamental groups of 3-manifolds which are extensions", Ann. of Math. (2) 95 (1972), 86-98.

[57] H. Hendriks, "Applications de la théorie d'obstruction en dimension 3", Bull. Soc. Math. France Mém. 53 (1977), 81-196.

[58] H. Hendriks, "Obstruction theory in 3-dimensional topology: an extension theorem", J. London Math. Soc. (2) 16:1 (1977), 160-164.

[59] H. Hendriks and F. Laudenbach, "Scindement d'une équivalence d'homotopie en dimension 3", Ann. Sci. École Norm. Sup. (4) 7 (1974), 203-217.

[60] J. A. Hillman, "Seifert fibre spaces and Poincaré duality groups", Math. Z. 190:3 (1985), 365-369.

[61] J. A. Hillman, "On 3-dimensional Poincaré duality complexes and 2-knot groups", Math. Proc. Cambridge Philos. Soc. 114:2 (1993), 215-218. 
[62] J. A. Hillman, "Embedding homology equivalent 3-manifolds in 4-space", Math. Z. 223:3 (1996), 473-481.

[63] J. A. Hillman, "Tits alternatives and low dimensional topology", J. Math. Soc. Japan 55:2 (2003), 365-383.

[64] J. A. Hillman, "Homomorphisms of nonzero degree between $\mathrm{PD}_{n}$-groups", J. Aust. Math. Soc. 77:3 (2004), 335-348.

[65] J. A. Hillman, “An indecomposable $\mathrm{PD}_{3}$-complex, II”, Algebr. Geom. Topol. 4 (2004), 1103-1109.

[66] J. A. Hillman, "PD 4 -complexes with free fundamental group", Hiroshima Math. J. 34:3 (2004), 295-306.

[67] J. A. Hillman, "An indecomposable $\mathrm{PD}_{3}$-complex whose fundamental group has infinitely many ends", Math. Proc. Cambridge Philos. Soc. 138:1 (2005), 55-57.

[68] J. A. Hillman, "Indecomposable $\mathrm{PD}_{3}$-complexes", Algebr. Geom. Topol. 12:1 (2012), 131-153.

[69] J. A. Hillman, “Indecomposable nonorientable $\mathrm{PD}_{3}$-complexes”, Algebr. Geom. Topol. 17:2 (2017), 645-656.

[70] J. A. Hillman, " $P D_{3}$-groups and HNN extensions”, 2020. arXiv 2004.03803

[71] J. A. Hillman, “ $P D_{3}$-pairs with compressible boundary”, 2020. arXiv 2006.08057

[72] J. A. Hillman and D. H. Kochloukova, "Finiteness conditions and $\mathrm{PD}_{r}$-group covers of $\mathrm{PD}_{n}$-complexes", Math. $Z$. 256:1 (2007), 45-56.

[73] J. A. Hillman and P. A. Linnell, "Elementary amenable groups of finite Hirsch length are locally-finite by virtuallysolvable", J. Austral. Math. Soc. Ser. A 52:2 (1992), 237-241.

[74] J. Howie, "On locally indicable groups", Math. Z. 180:4 (1982), 445-461.

[75] W. Jaco, "Heegaard splittings and splitting homomorphisms", Trans. Amer. Math. Soc. 144 (1969), 365-379.

[76] W. Jaco and P. B. Shalen, "Peripheral structure of 3-manifolds", Invent. Math. 38:1 (1976), 55-87.

[77] B. Jahren and S. Kwasik, "Three-dimensional surgery theory, UNil-groups and the Borel conjecture", Topology 42:6 (2003), 1353-1369.

[78] F. E. A. Johnson and C. T. C. Wall, "On groups satisfying Poincaré duality”, Ann. of Math. (2) 96 (1972), 592-598.

[79] J. Kahn and V. Markovic, "Immersing almost geodesic surfaces in a closed hyperbolic three manifold", Ann. of Math. (2) $\mathbf{1 7 5 : 3}$ (2012), 1127-1190.

[80] O. Kakimizu, "On maximal fibred submanifolds of a knot exterior", Math. Ann. 284:3 (1989), 515-528.

[81] M. Kapovich and B. Kleiner, "Coarse Alexander duality and duality groups", J. Differential Geom. 69:2 (2005), 279352.

[82] M. Kapovich and B. Kleiner, “The weak hyperbolization conjecture for 3-dimensional CAT(0)-groups”, Groups Geom. Dyn. 1:1 (2007), 61-79.

[83] A. Kawauchi, "Almost identical imitations of (3, 1)-dimensional manifold pairs", Osaka J. Math. 26:4 (1989), 743758.

[84] D. Kielak, "Residually finite rationally solvable groups and virtual fibring", J. Amer. Math. Soc. 33:2 (2020), 451-486.

[85] D. Kielak and P. H. Kropholler, "Isoperimetric inequalities for Poincaré duality groups", 2020. arXiv 2008.07812

[86] R. C. Kirby and L. R. Taylor, "A survey of 4-manifolds through the eyes of surgery", pp. 387-421 in Surveys on surgery theory, Vol. 2, Ann. of Math. Stud. 149, Princeton Univ. Press, 2001.

[87] J. R. Klein, L. Qin, and Y. Su, “On the various notions of Poincaré duality space”, 2019. arXiv 1901.00145

[88] D. H. Kochloukova, "Some Novikov rings that are von Neumann finite and knot-like groups", Comment. Math. Helv. 81:4 (2006), 931-943.

[89] D. H. Kochloukova and P. A. Zalesskii, "Tits alternative for 3-manifold groups", Arch. Math. (Basel) 88:4 (2007), 364-367.

[90] D. H. Kochloukova and P. A. Zalesskii, "Profinite and pro- $p$ completions of Poincaré duality groups of dimension 3", Trans. Amer. Math. Soc. 360:4 (2008), 1927-1949. 
[91] S. Kojima, "Finite covers of 3-manifolds containing essential surfaces of Euler characteristic = 0", Proc. Amer. Math. Soc. 101:4 (1987), 743-747.

[92] L. G. Kovács, "On finite soluble groups", Math. Z. 103 (1968), 37-39.

[93] P. H. Kropholler, “Cohomological dimension of soluble groups”, J. Pure Appl. Algebra 43:3 (1986), 281-287.

[94] P. H. Kropholler, "An analogue of the torus decomposition theorem for certain Poincaré duality groups", Proc. London Math. Soc. (3) 60:3 (1990), 503-529.

[95] P. H. Kropholler, "Baumslag-Solitar groups and some other groups of cohomological dimension two", Comment. Math. Helv. 65:4 (1990), 547-558.

[96] P. H. Kropholler, "A note on centrality in 3-manifold groups", Math. Proc. Cambridge Philos. Soc. 107:2 (1990), 261-266.

[97] P. H. Kropholler, "A group-theoretic proof of the torus theorem", pp. 138-158 in Geometric group theory (Sussex, 1991), vol. 1, London Math. Soc. Lecture Note Ser. 181, Cambridge Univ. Press, Cambridge, 1993.

[98] P. H. Kropholler and M. A. Roller, "Splittings of Poincaré duality groups", Math. Z. 197:3 (1988), 421-438.

[99] P. H. Kropholler and M. A. Roller, "Splittings of Poincaré duality groups, II", J. London Math. Soc. (2) 38:3 (1988), 410-420.

[100] P. H. Kropholler and M. A. Roller, "Relative ends and duality groups", J. Pure Appl. Algebra 61:2 (1989), 197-210.

[101] P. H. Kropholler and M. A. Roller, "Splittings of Poincaré duality groups, III", J. London Math. Soc. (2) 39:2 (1989), 271-284.

[102] I. J. Leary, "Uncountably many groups of type F P”, Proc. Lond. Math. Soc. (3) 117:2 (2018), 246-276.

[103] R. Lee, "Semicharacteristic classes", Topology 12 (1973), 183-199.

[104] A. Lubotzky and A. Mann, "Residually finite groups of finite rank", Math. Proc. Cambridge Philos. Soc. 106:3 (1989), 385-388.

[105] J. Luecke, "Finite covers of 3-manifolds containing essential tori”, Trans. Amer. Math. Soc. 310:1 (1988), 381-391.

[106] A. Margolis, "Groups of cohomological codimension one", 2019. arXiv 1908.08826

[107] B. Maskit, "A theorem on planar covering surfaces with applications to 3-manifolds", Ann. of Math. (2) 81 (1965), 341-355.

[108] G. Mess, "Examples of Poincaré duality groups", Proc. Amer. Math. Soc. 110:4 (1990), 1145-1146.

[109] G. Mess, "Finite covers of 3-manifolds and a theorem of Lubotzky", preprint, 1990.

[110] J. Milnor, "Groups which act on $S^{n}$ without fixed points", Amer. J. Math. 79 (1957), 623-630.

[111] W. D. Neumann, "Commensurability and virtual fibration for graph manifolds", Topology 36:2 (1997), 355-378.

[112] W. D. Neumann and F. Raymond, "Seifert manifolds, plumbing, $\mu$-invariant and orientation reversing maps", pp. 163196 in Algebraic and geometric topology (Santa Barbara, CA, 1977), Lecture Notes in Math. 664, Springer, 1978.

[113] W. D. Neumann and A. W. Reid, "Arithmetic of hyperbolic manifolds", pp. 273-310 in Topology '90 (Columbus, OH, 1990), Ohio State Univ. Math. Res. Inst. Publ. 1, de Gruyter, Berlin, 1992.

[114] J. Nicholson, “On CW-complexes over groups with periodic cohomology”, 2019. arXiv 1905.12018

[115] W. Parry, “A sharper Tits alternative for 3-manifold groups”, Israel J. Math. 77:3 (1992), 265-271.

[116] W. Plesken, "Minimal dimensions for flat manifolds with prescribed holonomy", Math. Ann. 284:3 (1989), 477-486.

[117] P. Przytycki and D. T. Wise, "Mixed 3-manifolds are virtually special", J. Amer. Math. Soc. 31:2 (2018), 319-347.

[118] A. Ranicki, "Algebraic and geometric splittings of the $K$ - and $L$-groups of polynomial extensions", pp. 321-363 in Transformation groups (Poznań, 1985), Lecture Notes in Math. 1217, Springer, 1986.

[119] L. Reeves, P. Scott, and G. A. Swarup, “A deformation theorem for Poincaré duality pairs in dimension 3”, 2020. arXiv 2006.15684 
[120] Y. W. Rong, "Degree one maps between geometric 3-manifolds”, Trans. Amer. Math. Soc. 332:1 (1992), 411-436.

[121] S. K. Roushon, "Vanishing structure set of 3-manifolds", Topology Appl. 158:6 (2011), 810-812.

[122] M. Sapir, “A Higman embedding preserving asphericity", J. Amer. Math. Soc. 27:1 (2014), 1-42.

[123] G. P. Scott, "Compact submanifolds of 3-manifolds", J. London Math. Soc. (2) 7 (1973), 246-250.

[124] P. Scott, "Normal subgroups in 3-manifold groups", J. London Math. Soc. (2) 13:1 (1976), 5-12.

[125] P. Scott, "Ends of pairs of groups", J. Pure Appl. Algebra 11:1-3 (1977/78), 179-198.

[126] P. Scott, "The geometries of 3-manifolds", Bull. London Math. Soc. 15:5 (1983), 401-487.

[127] P. Scott and G. A. Swarup, "Regular neighbourhoods and canonical decompositions for groups", Astérisque 289 (2003), vi+233.

[128] P. Scott and G. A. Swarup, “Canonical decompositions for Poincaré duality pairs”, 2007. arXiv 0703890v3

[129] D. Segal, “A footnote on residually finite groups”, Israel J. Math. 94 (1996), 1-5.

[130] P. B. Shalen and P. Wagreich, "Growth rates, $Z_{p}$-homology, and volumes of hyperbolic 3-manifolds", Trans. Amer. Math. Soc. 331:2 (1992), 895-917.

[131] T. Soma, "Virtual fibre groups in 3-manifold groups", J. London Math. Soc. (2) 43:2 (1991), 337-354.

[132] J. Stallings, "Homology and central series of groups", J. Algebra 2 (1965), 170-181.

[133] J. Stallings, "How not to prove the Poincaré conjecture", pp. 83-88 in Topology Seminar (Wisconsin, 1965), Ann. of Math. Stud. 60, Princeton Univ. Press, 1966.

[134] R. Strebel, "A remark on subgroups of infinite index in Poincaré duality groups", Comment. Math. Helv. 52:3 (1977), 317-324.

[135] R. G. Swan, "The nontriviality of the restriction map in the cohomology of groups", Proc. Amer. Math. Soc. 11 (1960), 885-887.

[136] R. G. Swan, "Periodic resolutions for finite groups", Ann. of Math. (2) 72 (1960), 267-291.

[137] G. A. Swarup, "On embedded spheres in 3-manifolds", Math. Ann. 203 (1973), 89-102.

[138] G. A. Swarup, “On a theorem of C. B. Thomas”, J. London Math. Soc. (2) 8 (1974), 13-21.

[139] L. R. Taylor, "Unoriented geometric functors", Forum Math. 20:3 (2008), 457-467.

[140] P. Teichner, "Maximal nilpotent quotients of 3-manifold groups", Math. Res. Lett. 4:2-3 (1997), 283-293.

[141] C. B. Thomas, "The oriented homotopy type of compact 3-manifolds", Proc. London Math. Soc. (3) 19 (1969), 31-44.

[142] C. B. Thomas, "Splitting theorems for certain PD $^{3}$-groups", Math. Z. 186:2 (1984), 201-209.

[143] B. Tshishiku and G. Walsh, "On groups with $S^{2}$ Bowditch boundary”, Groups Geom. Dyn. 14:3 (2020), 791-811.

[144] V. G. Turaev, "Fundamental groups of manifolds and Poincaré complexes", Mat. Sb. (N.S.) 110(152):2 (1979), 278296, 320. In Russian; translated in Math. USSR Sbornik 38 (1981), 255-270.

[145] V. G. Turaev, “Three-dimensional Poincaré complexes: classification and splitting”, Dokl. Akad. Nauk SSSR 257:3 (1981), 551-552. In Russian; translated in Soviet Math. Dokl. 23 (1981), 312-314.

[146] V. G. Turaev, "Fundamental groups of three-dimensional manifolds and Poincaré duality", pp. 231-238 in Topology (Moscow, 1979), Trudy Mat. Inst. Steklov. 154, 1983. In Russian; translated in Proc. Steklov Inst. (1984), 249-257.

[147] V. G. Turaev, "Three-dimensional Poincaré complexes: homotopy classification and splitting”, Mat. Sb. 180:6 (1989), 809-830. In Russian; translated in Math. USSR Sbornik 67 (1990), 261-282.

[148] C. T. C. Wall, "Finiteness conditions for CW-complexes", Ann. of Math. (2) 81 (1965), 56-69.

[149] C. T. C. Wall, "Finiteness conditions for CW complexes, II”, Proc. Roy. Soc. London Ser. A 295 (1966), 129-139.

[150] C. T. C. Wall, "Poincaré complexes, I", Ann. of Math. (2) 86 (1967), 213-245.

[151] C. T. C. Wall, "Periodic projective resolutions", Proc. London Math. Soc. (3) 39:3 (1979), 509-553. 
[152] C. T. C. Wall, "Poincaré duality in dimension 3", pp. 1-26 in Proceedings of the Casson Fest, edited by C. M. Gordon and Y. Rieck, Geom. Topol. Monogr. 7, Geom. Topol. Publ., Coventry, 2004.

[153] S. C. Wang, "The existence of maps of nonzero degree between aspherical 3-manifolds", Math. Z. 208:1 (1991), 147160.

[154] S. C. Wang, "The $\pi_{1}$-injectivity of self-maps of nonzero degree on 3-manifolds", Math. Ann. 297:1 (1993), 171-189.

[155] S. C. Wang and Y. Q. Wu, "Covering invariants and co-Hopficity of 3-manifold groups", Proc. London Math. Soc. (3) 68:1 (1994), 203-224.

[156] T. Weigel, “On profinite groups with finite abelianizations”, Selecta Math. (N.S.) 13:1 (2007), 175-181.

[157] F. Yu and S. Wang, "Covering degrees are determined by graph manifolds involved", Comment. Math. Helv. 74:2 (1999), 238-247.

[158] B. Zimmermann, "Das Nielsensche Realisierungsproblem für hinreichend große 3-Mannigfaltigkeiten", Math. Z. 180:3 (1982), 349-359. 



\section{Index}

Expressions beginning with Greek characters and other symbols are listed at the end of this index.

accessible (group), 4

adapted to $\Omega, 100$

admissible (graph of groups), 3

Algebraic Core Theorem, 79

Algebraic Loop Theorem, 36

algebraically $P^{2}$-irreducible, 71

almost coherent, 2

almost finitely presentable $\left(F P_{2}\right), 2$

ambient group (of $\mathrm{PD}_{n}$-pair), 9

anti-involution $\bar{g}$ of $\mathbb{Z}[G], 2$

ascendant, 91

atoroidal ( $\mathrm{PD}_{n}$-pair of groups), 9

augmentation $(\varepsilon: \mathbb{Z}[G] \rightarrow \mathbb{Z}), 2$

augmentation ideal $(I(G)), 2$

$\mathrm{BS}(p, q)$ (Baumslag-Solitar group), 1

Baumslag-Solitar relation, 105

Bieri theorems, 77, 78

Bieri-Eckmann Splitting Theorem, 79

Bieri-Strebel Theorem, 78

boundary compatibility, 31

boundary components, 9

Bowditch Theorem, 8

$c_{g}$ (conjugation by $g$ ), 1

$C_{G}(h)=C_{G}(\langle h\rangle), 1$

$C_{G}(H)$ (centralizer), 1

$c_{M}: M \rightarrow K\left(\pi_{1}(M), 1\right)$ (classifying map for $\left.\pi_{1}(M)\right), 5$

$C_{*}(X ; R[G / H])=C_{*}\left(X_{H} ; R\right), 5$

Cannon Conjecture, 74

Castel's Centralizer Theorem, 104

centre $(\zeta G), 1$

centralizer $\left(C_{G}(H)\right), 1$

Centralizer Condition, 43

Classification Theorem, 15, 28

cohomological period $\leqslant 4,47$

cohopfian, 125

commensurable, 1

commensurator $\left(\operatorname{Comm}_{G}(H)\right), 1$

commutator subgroup $\left(G^{\prime}\right), 1$

conjugate module $(\bar{M}), 2$

connected sum, 7, 20, 33

cube with handles, 37

$D(I(G) ; M)=\operatorname{Coker}\left(M^{\dagger}\right), 2$

$D I(G)=D(I(G) ; M), 2$

$D_{2 m}$ (dihedral group), 46
$D(G, \Omega)$ (double of $\mathrm{PD}_{3}$-pair), 79

$D_{\infty}=\mathbb{Z} / 2 \mathbb{Z} * \mathbb{Z} / 2 \mathbb{Z} \cong \mathbb{Z} \rtimes_{-1} \mathbb{Z} / 2 \mathbb{Z}$ (infinite dihedral group), 1

decomposes as a free product, 33

Decomposition Theorems, 33, 34

degree \pm 1 map, 7

degree of homomorphism, 79

dihedral group $\left(D_{2 m}\right), 46$

dualizing module $\left(H^{n}(G ; \mathbb{Z}[G])\right), 8$

$e(G)$, number of ends, 4

$E(\Gamma)$ (set of edges), 3

end module $\left(E(G)=H^{1}(G ; \mathbb{Z}[G])\right), 4$

End Module Theorem, 4

end of a tree, 39

equivariant diagonal approximation for $C_{*}, 2$

equivariant (co)homology, 5

$\mathrm{ev}_{M}: M \rightarrow M^{\dagger \dagger}, 2$

$F(r)$ (free group of rank $r$ ), 1

$\mathrm{FP}, \mathrm{FP}_{n}, 2$

$F^{r}\left(C^{*}\right)=C^{r} / \partial C^{r-1}, 18$

$\mathcal{F}_{H} G, 97$

finite ( $\mathrm{PD}_{n}$-space), 6

finiteness obstruction $(\sigma(P)), 23$

$H$-finite subset, 97

Fox-Lyndon matrix, 2

Fox-Lyndon complex, 5

$k$-free, 114

freely stably isomorphic, 3

fundamental class, 6

fundamental triple, 14

$G_{r}\left(C_{*}\right)=C_{r} / \partial C_{r+1}, 18$

$G$-tree, 39

$G$-tree module presented by $X, 40$

$G$-tree with $\infty$-vertices, 39

geodesic ray/line, 39

Gildenhuys-Strebel Theorem, 78

graph of groups $((\mathcal{G}, \Gamma)), 3$

Group Realization Theorem, 17

$h(G)$ (Hirsch length), 1

$H^{\circ}=C_{G}\left(C_{G}(H)\right), 85$

$H_{j}(X ; A)=H_{j}\left(A \otimes_{R[G / H]} C_{*}(X ; R[G / H])\right)$ (equivariant homology), 5

$H^{n-j}(X ; B)$ (equivariant cohomology), 5

$H^{+}=H \cap \pi^{+}, 7$

$H^{k}(G, \Omega ; M), 9$

Hirsch length $(h(G)), 1$ 
Hirsch-Plotkin radical $\sqrt{G}, 1$

hopfian, 125

$I(G)$ (augmentation ideal), 2

I-bundle type, 80

indecomposable (graph of groups), 3

indecomposable ( $\mathrm{PD}_{3}$-complex), 20

indicable, 108

induced $\mathrm{PD}_{n}$-pair $\left(\left(H, \Omega_{H}\right)\right), 9$

JSJ Decomposition Theorem, 100

$\mathrm{Kb}=\mathbb{Z} \rtimes_{-1} \mathbb{Z}$ (Klein bottle group), 1

Kropholler-Roller theorems, 98

large, 117

loop isomorphism, 3

Lyndon-Hochschild-Serre spectral sequence (LHSSS), 2

malnormal subgroup, 98

max-c (maximum condition on centralizers), 1

metacyclic group, 46

nontrivial Baumslag-Solitar relation, 105

normalizer $\left(N_{G}(H)\right), 1$

Normalizer Condition, 1

open $\mathrm{PD}_{n}$-group, 9

order of a group $(|G|), 1$

orientable (subgroup), 7

orientable ( $\mathrm{PD}_{n}$-group), 8

orientation character $\left(w: G \rightarrow \mathbb{Z}^{\times}\right), 6,8$

origin function $(o: E(\Gamma) \rightarrow V(\Gamma)), 3$

$p^{2}$ condition, 46

$p q$-condition, 46

$P^{+}=P_{\pi}$ (orientable covering), 7

$\mathrm{PD}_{n}$-complex, 6

$\mathrm{PD}_{n}$-group, 8

$\mathrm{PD}_{n}^{+}$-group, 8

$\mathrm{PD}_{n}$-pair, 8

$\mathrm{PD}_{n}$-pair of groups, 9

$\mathrm{PD}_{n}$-space, 6

PD Extension Theorem, 78

periodic cohomology, 46

peripheral system, 27

Poincaré duality of dimension $n$ with orientation character $w, 6$

Poincaré-Lefschetz duality, 8

polycyclic, 1

pro- $p$ completion, 109

profinite completion, 109

projectively homotopic, 3 projective homotopy equivalence, 3

projectively stably isomorphic, 3

proper (connected sum), 20

proper (JSJ decomposition), 101

properly locally cyclic, 114

Prüfer rank $(u(G)), 112$

$\operatorname{rank}(\operatorname{rk}(G)), 112$

Realization Theorem, 19, 31

reduced (graph of groups), 3

reduced ( $\mathrm{PD}_{3}$-pair), 27

residually finite, 109

restrained, 108

satisfy a nontrivial Baumslag-Solitar relation, 105

sectional $p$-rank $\left(u_{p}(G)\right), 112$

Seifert type, 9

Seifert 3-manifold group, 80

simple ( $\mathrm{PD}_{n}$-complex), 7

$\operatorname{sing}(H), 97$

splits over a subgroup, 4

Splitting Theorem, 20

$S Q$-universal, 117

stably isomorphic, 3

standard ( $\mathrm{PD}_{n}$-complex), 7

$\operatorname{Star}(v), 100$

Strebel Theorem, 78

subnormal, 92

Swan subgroup, 51

$\mathrm{TL}(2, p), 47$

target function $(t: E(\Gamma) \rightarrow V(\Gamma)), 3$

Tarski monster, 75, 115

terminal $G$-tree, 39

Tits alternative, 107

2-Torsion Central Theorem, 48

Turaev condition, 30

Turaev's Realization Theorem, 19

$u(G)$ (Prüfer rank), 112

$u_{p}(G)$ (sectional $p$-rank), 112

Unique Factorization Theorem, 22

universal coefficient spectral sequence, 5

$v \beta(G), 5$

$v \beta(G ; p), 5$

$V(\Gamma)$ (set of vertices), 3

virtually has some property, 1

volume condition, 125

$\mathcal{X}$ (class of groups), 4

$X^{[k]}$ (k-skeleton), 5

$X_{H}$ (associated covering space), 5 
$\mathbb{Z} * q=\mathrm{BS}(1, q), 1$

$\mathbb{Z}^{w}, 2$

$\mathbb{Z} / 2 \mathbb{Z}^{-}$(subgroup of order two on which $w \neq 1$ ), 7

Greek characters

$\beta_{i}(X ; F)=\operatorname{dim}_{F} H_{i}(X ; F), 5$

$\beta_{i}(X)=\beta_{i}(X ; \mathbb{Q}), 5$

$\Delta(G, \Omega)=\operatorname{Ker}\left(\varepsilon_{W}\right), 9$

$\varepsilon: \mathbb{Z}[G] \rightarrow \mathbb{Z}$ (augmentation), 2

$\zeta G$ (centre of group), 1

$v_{C_{*}, r}, 19$

$v_{G}$ (norm element in $\left.\mathbb{Z}[G]\right), 46$

$\pi^{+}=\operatorname{Ker}(w), 7$

$\pi \mathcal{G}, 3$

$\pi K=\pi_{1}\left(S^{3} \backslash K\right)$ (knot group), 1

$\pi_{1}$-injective, 27

$\sigma(P)$ (finiteness obstruction), 23

$\chi^{\text {virt }}(G)=\chi(K) /[G: K]$ (rational Euler characteristic), 2

$\xi(G), 40$
Other symbols

$\langle S\rangle$ (subgroup generated by $S$ ), 1

$\langle S\rangle\rangle($ normal closure of $S), 1$

$\bar{g}=w(g) g^{-1}$ (anti-involution), 2

$\bar{M}$ (conjugate module), 2

$M^{\dagger}=\overline{\operatorname{Hom}_{R}(M, R)}, 2$

$[M]$, (free stable isomomorphism class), 3

$[M]_{\mathrm{pr}}$ (projective stable isomomorphism class), 3

$[M, N]$ (homotopy classes of module homomorphisms), 3

$G^{\prime}$ (commutator subgroup), 1

$\sqrt{G}$ (Hirsch-Plotkin radical), 1

$|G|$ (order of $G$ ), 1

$\widehat{G}$ (profinite completion), 109

$\widehat{G}_{p}$ (pro- $p$ completion), 109

$\widetilde{X}$ (universal covering space), 5

$\left[\left\{\kappa_{i}\right\}, w, \mu\right], 28$

$[\pi, w, \mu], 14$

$\partial P$ (boundary of $\mathrm{PD}_{n}$-pair), 8

д $\widetilde{P}$ (preimage of $\partial P$ in $\widetilde{P}$ ), 8

$\infty$-vertex (of $G$-tree), 39 




\section{THE OPEN BOOK SERIES 3 \\ Poincaré Duality in Dimension 3}

Poincaré duality is central to the understanding of manifold topology. Dimension 3 is critical in various respects, being between the known territory of surfaces and the wilderness manifest in dimensions $\geq 4$. The main thrust of 3-manifold topology for the past half century has been to show that aspherical closed 3-manifolds are determined by their fundamental groups. Relatively little attention has been given to the question of which groups arise. This book is the first comprehensive account of what is known about $\mathrm{PD}_{3}$-complexes, which model the homotopy types of closed 3-manifolds, and $\mathrm{PD}_{3}$-groups, which correspond to aspherical 3-manifolds. In the first half we show that every $P^{2}$-irreducible $\mathrm{PD}_{3}$-complex is a connected sum of indecomposables, which are either aspherical or have virtually free fundamental group, and largely determine the latter class. The picture is much less complete in the aspherical case. We sketch several possible approaches for tackling the central question, whether every $\mathrm{PD}_{3}$-group is a 3-manifold group, and then explore properties of subgroups of $\mathrm{PD}_{3}$-groups, unifying many results of 3-manifold topology. We conclude with an appendix listing over 60 questions. Our general approach is to prove most assertions which are specifically about Poincaré duality in dimension 3, but otherwise to cite standard references for the major supporting results.

Target readership: Graduate students and mathematicians with an interest in low-dimensional topology. 